\title{
HASL - 93
}

UC 41, Health \& Safety

TID-4500, 15 th Ed.

\author{
SUMMARY OF GUMMED FILM RESULTS \\ THROUGH DECEMBER, 1959
}

John H. Harley

Naomi A. Hallden

Long D. Y. Ong

Health and Safety Laboratory

This document is

September $5, \quad 1960$

PUBLICLY RELEASABLE

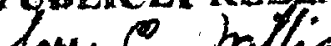

Laxe ece palhani

Authorizing Official

Date $06 / 23 / 2006$

U. S.: ATOMIC ENERGY COMMISSION

New York Operations Office

Health and Safety Laboratory 


\section{DISCLAIMER}

This report was prepared as an account of work sponsored by an agency of the United States Government. Neither the United States Government nor any agency Thereof, nor any of their employees, makes any warranty, express or implied, or assumes any legal liability or responsibility for the accuracy, completeness, or usefulness of any information, apparatus, product, or process disclosed, or represents that its use would not infringe privately owned rights. Reference herein to any specific commercial product, process, or service by trade name, trademark, manufacturer, or otherwise does not necessarily constitute or imply its endorsement, recommendation, or favoring by the United States Government or any agency thereof. The views and opinions of authors expressed herein do not necessarily state or reflect those of the United States Government or any agency thereof. 


\section{DISCLAIMER}

Portions of this document may be illegible in electronic image products. Images are produced from the best available original document. 
This report was prepared as an account of Governinent sponsored work. Neither the United States, nor the Commission, nor any person acting on behalf of the Commission.

A. Makes any warranty or representation, expressed or implied, with respect to the accuracy, completeness, or usefulness of the information contained in this report, or that the use of any information, apparatus, method, or process disclosed in this report may not infringe privately owned rights; or

B. Assumes any liabilities with respect to the use of, or for damages resulting from the use of any information, apparatus, method, or process disclosed in this report.

As used in the above, "person acting on behalf of the Commission" includes any employee or contractor of the Commission, or employee of such contractor, to the extent that such employee or contractor of the Commission, or employee of such contractor prepares, disseminates, or provides access to, any information pursuant to his employment or contract with the Commission, or his employment with such contractor.

This report has been reproduced directly from the best available copy.

Printed in USA. Price \$3.50. Available from the Office of Technical Services, Department of Commerce, Washington 25, D. C. 


\section{ABSTRACT}

The data for gummed film fallout measurements through December 1959 are reported. While the initial purpose of the gummed film network was to determine the geographic distribution and time of arrival of fallout, considerable effort has been devoted to computation of strontium 90 deposition and infinity gamma dose. These latter computations have been satisfactory for the period of observation and furnish data for locations not covered by other types of measurement.

Detailed comparisons are made between strontium 90 estimates from gummed film and analyses made on pot and soil samples. Comparable tests of the gamma dose estimate are not possible.

The bulk of the report consists of summary tables for each gummed film station listing the monthly estimates of strontium 90 deposition and infinity gamma dose. 


\section{ACKNOWLEDGEMENTS}

The authors wish to acknowledge the assistance of the many people who have worked on this program since its inception. It is impossible to name all the individuals involved but our particular thanks are given to the observers of the various U.S. Weather Bureau Stations, the Canadian Meteorological Service, the Air Weather Service of the Air Force, and to the others who collected the samples over this period of years.

In the Health and Safety Laboratory we are grateful to the gummed film staff for preparing and counting the enormous number of samples involved and to the staff of the Stetistics Branch for handing the card punching, computation and printing of data. The strontium 90 analjses which have been used for comparison were made under the gereral direction of Edward $P$. Hardy, Jr. and Gerald Hamada.

Considerable general assistance has been available from Dr. Lester Machta and Robert List of the U. S. Weather Bureau who arranged for many of the sampling locations and maintained liaison between the Weather Bureau and our Laboratory.

Dr. S. Allan Lough, Director of the Jealth and Safety Laboratory, made many valuable suggestions in the preparation of the manuscript. 


\section{CONTENTS}

\section{$\underline{\text { Page }}$}

ABSTRACT

ii

ACKNOWLEDGEMENTS

SECTION I

INTRODUCTION

Historical

The Computation Model

Defects of System

1

5

7

EXPERIMENTAL DATA

10

Comparison of Duplicate Stations

11

Evaluation of Strontium 90 Data

14

Evaluation of Infinity Gamma Dose Data

SUMMARY

30

PROCEDURE FOR GUMMED. FILM

32

Sampling

Sample Preparation

Counting

Data Handling

BIBLIOGRAPHY

SECTION II

MONTHLY SUMMARIES FOR INDIVIDUAL STATIONS 
For many years, gummed film sampling comprised the only widespread network for measuring fallout. This technique fulfilled its original purposes of delineating the geographic distribution of fallout and indicating the time of arrival of radioactive material. While the test pattern remained simple and included only fission devices, it was also possible to estimate individual long-lived nuclides such as $\mathrm{Sr}^{90}$ and to estimate the fallout infinity gamma dose.

More complex test patterns and delayed fallout from thermonuclear devices made the $\mathrm{Sr} 9 \mathrm{C}$ and gamma dose estimates very difficuIt. Despite the complexities, considerable effort has been devoted to testing various mathematical models to allow computation of these two parameters and thus to extract the utmost from the available data. Tis report describes the model adopted and gives the results of these computations jin some detail. In addition, comparisons have been made with more direct measurements of Sr 90 fallout where these are availabie.

The data indicate that useful information on $\mathrm{Sr}^{90}$ deposition has been obtained by the gumned fijm technichie. rine gamma dose estimates cannot be confirmed, but are the best availabie for any network.

\section{Historical}

The first detection of radicactive fallout away from the site of the explosion was in Rochester, New York, in Eebruary of 1951, although Webb (I) had reported activity of urinown origin in paperboard in 1945. The Health and Safety Laboratory was subsequently given the responsibility of determining the relative fallout at distances beyond 200 miles from the Nevada test site. Later tinis responsibility ras modified to delineating world-wide fallout from the megaton weapons fired in the Pacific and elsewhere. 
The original measurements were extremely simple as only the total mixed fission product beta activity was required. These data gave information on the arrival of fallout and to some extent on the relative fallout contribution at different locations.

In the first network of 10 stations set up for the United States in the spring of 1951, the collector was a metal tray having an area of about 9 square feet. A layer of water was maintained in the tray and every twentyfour hours the contents were filtered to obtain the particulate activity. The water, including any rainfall, was collected in a polyethylene bottle. These samples were prepared for beta counting and measured with Geiger tubes against Radium D+E standards.

For the falI 1951 tests the retwork was expanded to 61 stations and 20 of these had gummed paper samplers as wel.. as trays. These stations were operated for the Atomic Energy Comission by the U. S. Weather Bureau. The gummed papers were forwarded to the Lealth and Safetiy Laboratory for ashing and counting. The results on these stations were reported by the Health and Safety Laboratory in 1952. (2)

The network in the United States for tre spring 1952 tests included 109 deposition stations with gunired paper samplexs. The data were reported in 1953 by Eisenbud and Fariey. (3)

During the fall 1952 Pacific tests the network consisted of 51 gummed paper stations in the continental inited States and 60 elsewhere in North America and overseas. All later sampings were with a gummed acetate film instead of gummed paper which gave a smaller amount of ash. In the spring 1953 Nevada series, 96 U. S. stations and 29 others were in operation, and in the spring 1954 Pacific series, the network was $39 \mathrm{U}$. S. stations plus 
81 others. These data were also reported by Eisenbud and Harley. $(4,5)$

The network since 1954 has been quite stable. In January 1959 it

consisted of $46 \mathrm{U}$. S. stations, 68 elsewhere in North America and overseas and 64 maintained in cooperation with other countries through the United Nations.

As the approach to fallout problems became more sophisticated, it was desirable to have a measure of individual radionuclides, particularly $\mathrm{Sr} 90$ and to have an estimate of the gamma dose delivered to the population in various geographic areas. Actual $\mathrm{Sr}^{90}$ measurements could best be performed on monthly samples but, because of the radiochemistry involved, this collection system grew very slowly. It was apparent that it would be very useful if a reasonable estimate of the $\mathrm{Sr} 90$ deposition could be made from the total beta measurements on gummed film.

In the period before megaton weapons were fired, the radioactive debris stayed in the troposphere and was cleared out with an average life of about one month. Thus, it was usually possible to attribute fallout to a single burst and to make reasonable estimates of the $\mathrm{Sr} 90$ content based on total beta measurements. It was also possible by a knowledge of the nuclides produced, to estimate the infinite plane gamma dose.

The original calculations of $\mathrm{sr}^{90}$ deposition from measurements of total beta activity on ashed gummed-film samples were performed as follows:

1. The activity measured on a given sampling day was attributed to the test immediately preceding that sampling day.

2. The measured activity on the counting day was extrapolated to a fixed day (usually the first day of the following year) by the formula 


$$
\begin{aligned}
A=A_{C}\left[\frac{C-B}{E-B}\right]^{1.2} \\
\text { where, } \\
A_{C}=\beta \text { activity on counting day } \\
C=\text { Counting day } \\
B=\text { Burst day } \\
E=\text { Extrapolation day }
\end{aligned}
$$

3. The $\mathrm{Sr}^{90}$ fraction of the total beta activity on this day was taken from modified Hunter and Ballou(6) curves.

4. The $\operatorname{sr} 90$ activity values for the individual days were summed by months and these sums added for the desired period. The same data were also used to compute an infinity gamma dose using the calculation system described by Lallden and Harley. (7)

The data reported for fallout through September 1955 (5) were based on the above computations, but comparison of the estimated $\mathrm{Sr}^{90}$ values with available radiochemical data showed the method was inadequate. The actual fallout was older and contained a higher percentage of $\mathrm{Sr}^{90}$ than the model indicated.

A new model was then devised to take stratospheric debris into account. Tests of this model appeared to give reasonable agreement between estimated and measured $\mathrm{Sr}^{90}$. This was the genergl system:

1. Estimates of the yields of total fission products and of $\mathrm{Sr}^{90}$ were obtained for each weapon test.

2. The total fission-product yield for each test was added to the calculated fission-product residue from previous tests. (The $\mathrm{T}^{-1.2}$ law was used for decaying total fission product activity.)

3. The combined fissionmproduct yield was used to estimate an effective burst date. This was used with samples following each test to extrapolate from counting date to a fixed computing date.

4. The $\mathrm{Sr}^{90}$ activity from each test was added to the accumulated $\mathrm{Sr}^{90}$ from previous tests. (ivo $\mathrm{Sr} 90$ decay was included)

5. The $\mathrm{Sr}^{90}$ to fission-product beta activity ratio was computed and applied to the measured beta activity on each sample. 
These data were reported by Eisenbud and Harley $(8)$ covering fallout through June 1957. The model was applied only to the results obtained after May 1956.

Unfortunately, later calculations have cast doubt on the validity of the effective burst date concept and actual errors of computation have been uncovered. The present model was designed to overcome some of the objections to the previous one. All of the data since the beginning of 1954 have been recomputed according to this third model.

It would have been. technically possible to introduce a number of experimentally determined refinements into the model, but the cost of computation for some 375,000 pieces of data would have been prohibitive. Therefore, the relatively simple model described had to be accepted.

\section{The Computation Model}

The present model was designed to take into account new data on $\mathrm{Sr} 90$ Vield, stratospheric and tropospheric residence times and the decay of $\mathrm{Sr}^{90}$. The model will first be outlined and then its defects will be discussed.

1. An estimate of the fission yield is obtained for each weapon test.

2. The value of 133 megacuries of beta activity per nominal bomb is used to obtain total megacuries of fission products at one day.

3. The value of 0.00105 percent of $\mathrm{Sr}^{90}$ is used to obtain total megacuries of $\mathrm{Sr} 90$ at one day.

4. The megacuries of mixed fission products produced in each test are decayed by the $\mathrm{T}^{-1.2}$. Iaw for each subsequent day and the results for a.l1 tests summed.

5. The megacuries of $\mathrm{Sr} 90$ produced in each test are decayed with a half-life of 27.7 years for each subsequent day and the results for all tests summed.

6. Both the mixed fission products and $\mathrm{Sr} 90$ are also corrected for tropospheric $(<3 / 4 \mathrm{MT})$ and stratospheric $(>3 / 4 \mathrm{MT})$ deposition using half-residence times of 20 days and 4 years respectively. 
7. The mixed fission products existing on sampling day after decay and deposition corrections are further decayed to counting day.

8. The ratio of mixed fission products on sampling day (from all tests before sampling day) to that on counting day is used to correct the beta activity of individual samples back to sampling day.

9. The ratio of $\mathrm{Sr} 90$ to mixed fission products present from all tests on sampling day is used to compute the $\mathrm{Sr}^{90}$ deposition from the individual samples.

10. The daily values are summed to give the total deposition for the month. This value is divided by the number of actual samples in the month and multiplied by the expected number to give the final value for monthly Sr90 deposition. This value is as of the sampling month.

11. The computed beta activity on sampling day is treated by the method of Hallden and Harley(7) to obtain the infinity gamma dose from fallout on that day. This ealculation takes into account the apportionment of the computed beta activity anong the debris of different ages.

12. The daily gamma values are surmed and corrected as for the monthly Sr90 deposition.

The mathematical expressions are:

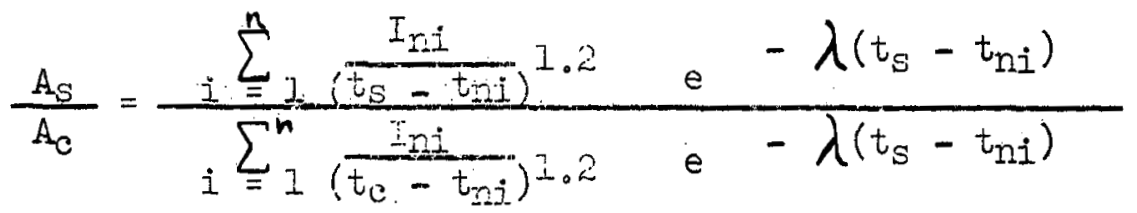

$$
\begin{aligned}
& A_{S}=\beta \text { Activity on sampling aay dpm } / f t^{2} \\
& A_{c}=\beta \text { Activity on counting day } \mathrm{dpm} / \mathrm{ft}^{2} \\
& i=\text { Eurst number } \\
& I_{n i}=\begin{aligned}
\text { Zield for hurst } j, \text { Me of mixed fission } \\
\text { products at } 1 \text { day }
\end{aligned} \\
& t_{S}=\text { Sampling day } \\
& t_{n i}=3 u r s i d a y \\
& \lambda=\text { Deposition constant }
\end{aligned}
$$




$$
\operatorname{Sr}^{90}=\frac{A_{S}}{80} \cdot \frac{1.05 \times 10^{-5}}{D} \sum_{i=1}^{n} I_{n i} e^{-\left(\lambda+6.8 \times 10^{-5}\right)\left(t_{S}-t_{n i}\right)}
$$

Where

$$
\begin{aligned}
& D=\sum_{i=1}^{n} I_{n i} \frac{1}{\left(t_{s}-t_{n i}\right)^{1.2}} e^{-\lambda\left(t_{s}-t_{n i}\right)} \\
& \mathrm{Sr}^{90}=\mathrm{mc} / \mathrm{mi}^{2} \text { of } \mathrm{sr} 90 \\
& \lambda=3.5 \times 10^{-2} \text { for } I_{n i} \quad 5000 \text { (Tropospheric) } \\
& \lambda \quad 4.7 \times 10^{-4} \text { for } I_{n i} \quad 5000 \text { (Stratospheric) } \\
& \text { Dose }=A_{s} \frac{3.0 \times 10^{-6}}{D} \quad \sum_{i=1}^{n} \quad I_{n i} \frac{1}{\left(t_{s}-t_{n i}\right) \cdot 425^{e}}-\lambda\left(t_{s}-t_{n i}\right) \\
& \text { Dose }=\text { Infinity } \gamma \text { dose in millirads }
\end{aligned}
$$

\section{Defects of System}

There are a number of defects in the overall gummed film system and in fact, it only deserves the effort expended because there were no other types of sampling available for the time and locations at which most of these measurements were made. These defects will be discussed individually even though it is not possible to give good numerical estimates of the errors involved.

1. The measurement is a total beta count only and is subject to the errors of self-absorption and the change in average energy of mixed fission products with time. The self-absorption is partially corrected for by using basic standards. with the same weight of material as found in the average fallout sample. No correction is made for such heavy or light samples as may appear from time to time. The average energy of the mixed fission products has been calculated in this Laboratory based on the assumptions that the average energy is one-third the maximum energy of the beta particle and that the percentage of each isotope in fallout samples follows the Hunter and Ballou (6) calculations.

The average energies calculated on this basis are: 


$$
\begin{array}{cc}
10 \text { days } & 0.32 \mathrm{Mev} \\
80 \text { days } & 0.30 \mathrm{Mev} \\
150 \text { days } & 0.28 \mathrm{Mev} \\
2 \text { years } & 0.48 \mathrm{Mev}
\end{array}
$$

The average energy of potassium 40 according to Marinelli, Brinkerhoff and Hine $(10)$ is $0.40 \mathrm{Mev}$. We believe this to be the best approximation of the energy for mixed fission products among the available long-lived isotopes.

2. There are losses both in the collection process and in the sample processing. These are corrected for only by the overall factor of 1.6 relating mixed fission product $\beta$ activity found in pot samples to that found in composite gummed film samples for the same periods. This factor of 1.6 was shown to be a good average value for New York City over a period of two years in 1954 and 1955, byt does not necessarily apply to each individual sample. Welford $(11)$ has shown the relative loss of several nuclides and has found a factor of 1.85 for the year 1958 .

3. The general formula depends on the $T^{-1.2}$ law which is not absolutely applicable to each sample and each type of debris. On the average, however, for fallout remote from the test site, it is the best value presently available. (Later reports, such as NRDL-247 indicate an exponent of -3.25 which is not appreciably different.)

4. No allowance is made for fission product fractionation or for induced activities. The fractionation is probably less critical at considerable distances from the test site but radiochemical data are not available for cheoking this point. Most of the induced activities are of relatively short half-life and are not counted with the time lag inherent in the system.

5. The general formula does not allow for differences in geographic distribution of the debris. (For instance the 1958 USSR polar tests did not appear in the southern hemisphere). All samples are treated by the same formula. The effect of this can only be shown by comparing computed $\mathrm{Sr} 90^{\circ}$ values with the few data available for measured Sr 90 deposition.

6. The general formula does not allow a time lag for the debris to reach different geographic areas. The inclusion of such a lag would have made the computation impossibly. complex.

7. The general formula does not allow for distribution of megaton debris between the stratosphere, troposphere and local deposition. This could have been included but would have essentially doubled the computation. This was not considered worth while since the estimates of this distribution ere of doubtful validity. 
8. The general formila does not allow for the cases when almost all of the debris came from a single burst. The individual daily results, however, have been reviewed. Where the presence of fallout, known trajectories and supplementary data indicate that the fallowt does result largely from a single burst, corrections have been made individually. This, of course, results in decreasing the $\mathrm{sr}^{90}$ and gamma dose values for these particular samples. Since only markedly high values can be detected, the process may destroy the relationship between stations so corrected and those not corrected. 


\section{EXPERIMENTAL DATA}

For the most part, the experimental data are derived from the average of duplicate daily gummed film samples analyzed as described in the section on Procedure. The monthly summaries of estimated $\mathrm{Sr}$ ? deposition and infinity gamma dose make up the bulk of this report, but certain data have been extracted in this section to compare with more direct measurements or with other estimates. The evaluations are separated into comparison of duplicate stations, $\mathrm{Sr}^{90}$ data and gamma dose data. There are certain cases where daily data are not available. Since these may, enter into the evaluations attempted, they are described below.

During certain periods in 1955 and 1956, the daily gurmed film samples were composited on a monthly basis for counting. The purpose of this compositing was not to reduce the counting load but was an attempt to collect sufficient gummed film ash to make a radiochemical analysis for $\mathrm{Sr}^{90}$. This effort was not successful as it was found that the collection efficiency for Sr 90 was considerably poorer than the efficiency for the total mixed fission products. This was later confirmed by Welford ${ }^{(11)}$ in his measurements on New York City samples.

The composite samples have been treated in the computation process as though all activity had been deposited on the 15 th of the sampling month. This should not lead to any marked computation errors since the compositing was only carried out during periods or at stations where local fallout was not expected. All composite samples are marked as such in the summary tables for each station.

Because of the large number of samples belng handled and because of difficulties in the field, there are not always sufficient daily samples 


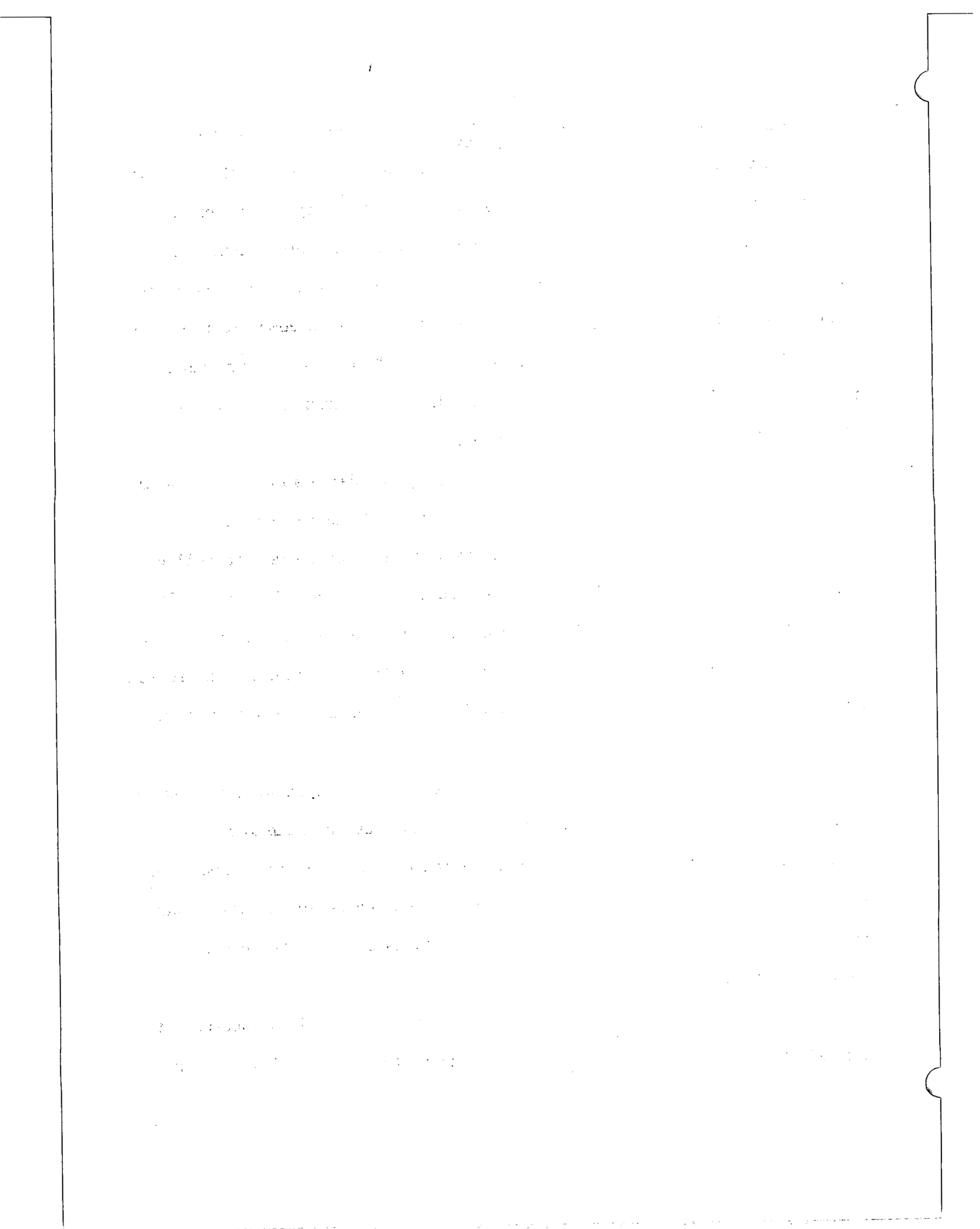


to justify a monthly summary. In the data tables, we have arbitrarily taken the reporting of less than 10 days in the month to be an insufficient sample. As it is desirable to obtain an estimate of cumlative values for comparison With soil data and to approximate the total deposition where other data are lacking, missing months have been filled in by geographic interpolation. In the arbitrary system selected, the three closest stations are averaged for geographic interpolation with some weight being given to the months immediately before and after the missing month. These interpolated estimates are marked as such in the summary tables for each station. Comparison of Duplicate Stations

Since duplicate films were exposed daily at most of the network stations, there is a considerable body of data on paired samples at the same location. A statistical treatment of the duplicates is planned as an exercise in methematical statistics, but this project will not be complete for some time. It might be noted that visual inspection shows remarkable agreement for the most part. This may be taken as an indication that we are usually dealing with a statistically valid number of particles deposited on each film.

It is possible to compare results from two stations in the same city for a few cases. Such data have been tabulated for New York City (Table I) and for Tokyo and Durban (Table 2), Whlle it is not necessarily true that such paired stations will give identical results they should be close.

The tables indicate a number of differences, but the overall agreement is reasonable, It is not possible to attribute errors individually to sampling, analysis, or computation. 


\section{TABLE I}

Comparison of Two Gumed Film Stations at New York City, HASL (136) and La Guardia Airport (117) $\mathrm{mc} / \mathrm{mi}^{2}$ of Strontium 90

\begin{tabular}{|c|c|c|c|c|c|c|}
\hline \multirow[b]{2}{*}{ Month } & \multicolumn{2}{|c|}{1954} & \multicolumn{2}{|c|}{1955} & \multicolumn{2}{|c|}{1958} \\
\hline & 136 & .177 & 136 & 116 & 136 & 117 \\
\hline 1 & - & - & .12 & $.03 *$ & - & - \\
\hline 2 & .51 & .05 & .33 & .27 & .34 & .36 \\
\hline 3 & .07 & .04 & - & - & .76 & .81 \\
\hline 4 & .09 & .05 & - & - & 2.69 & 2.03 \\
\hline 5 & .22 & .06 & - & - & .72 & .82 \\
\hline 6 & .09 & .07 & .92 & .68 & .64 & .56 \\
\hline 7 & .06 & .06 & .27 & $.10 *$ & .36 & .73 \\
\hline 8 & .11 & .08 & .38 & $10 *$ & .20 & .25 \\
\hline 9 & .27 & .26 & .23 & $.11 *$ & .41 & .32 \\
\hline 10 & .10 & .10 & .20 & $26 *$ & .72 & 87 \\
\hline 11 & 1.44 & .47 & .13 & .25 & .78 & .75 \\
\hline 12 & .14 & $.08 *$ & .20 & .16 & .86 & .63 \\
\hline
\end{tabular}

* Composite Sample

- Data missing for HASI Station (136) 


\section{TABLE 2a}

Comparison of Two Gummed Film Stations at Tokyo $\mathrm{mc} / \mathrm{mi}^{2}$ of Strontium 90

$\begin{array}{cccc}\text { Year } & \text { Station } 805 & \text { Station 820 } \\ 1956 & 11 & 0.33 & 0.21 \\ & 12 & 0.20 & 0.22 \\ 1957 & 1 & 0.27 & 0.21 \\ & 2 & 0.75 & 0.53 \\ & 3 & 0.70 & 0.55 \\ 4 & 1.13 & 3.59 \\ & 5 & 0.90 & 1.23 \\ 6 & 0.58 & 0.53 \\ & 7 & 0.86 & 1.21 \\ 8 & 0.35 & 0.37 \\ & 9 & 0.61 & 0.87\end{array}$

TABLE 2B

Comparison of Two Gummed Film Stations at Durban $\mathrm{mc} / \mathrm{mi}^{2}$ of Strontium 90

Station 713

Year

1957

1958
Month

6
7
8
9
10
11
12

6
7

8

10

11

(1)

1

3

4

5

6

7

8

9

10

11
Univ, of Durban

0.04

0.13

0.20

0.20

0.21

0.08

0.07

0.08

0.03

0.01

0.01

0.10

0.03

0.07

0.05

0.18

0.10

0.09

0.09
Station 723

Natal Airport
0.06
0.19
0.29
0.23
0.05
0.08
0.22

0.11

0.22

0.47

0.01

0.18

0.03

0.05

0.19

0.19

0.16

0.03

0.07 


\section{Evaluation of $\operatorname{Sr} 90$ Data}

In estimating the validity of gummed film values for $\mathrm{Sr} 90$, we have available radiochemical $\mathrm{Sr}^{90}$ measurements in monthly pot samples and in soil samples. The earliest useful pot data began in April 1954 at New York City but even as late as January 1957, only a few stations were available. For the soil collections, a few locations were sampled in October of 1955 and a larger number in 1956 and 1958.

Pot samples, like gummed film, are a measure of fallout rate. The analyses are available on a monthly basis and no decay corrections are required.

The comparison of calculated and measured values must be made with a degree of caution and the pot values should not be automatically assumed as correct. In all of the comparative tables we have indicated whe re certain pot values are suspect. Suspicion is raised where other radiochemical data, such as Strontium-89/90 ratios indicate possible error in the $\mathrm{Sr} 90$ analysis. It is also possible, of course, that other pot samples may have suffered losses in the sample transfer without disturbing the $89 / 90$ ratio. There is a possible added factor in that many pot-gummed film comparisons are inade for cases when the two collectors are separated by some distance even though in the same city.

Three sets of data are presented for New York City. Table 3 shows the pot data and the computed $\mathrm{Sr} 90$ values based on the original single burst assumption for several months in 1956. The computed $\mathrm{Sr}^{90}$ is obviously very low and the apparent age of the fallout is much greater than was assumed. This disagreement was the reason for seeking a better model. Table 4 compares pot values with $\mathrm{Sr} 90$ computed by the present model. Inspection of this table brings out several points: 
TABLE 3

Comparison of Pot Analyses With Single Burst Gurmed Film Estimates of $\mathrm{Sr} 90$ (New York City)

\section{Sampling Period}

May 1956

June

July

August

September

October

November

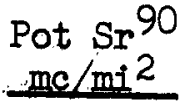

0.83

.63

.72

.53

.32

.54

.51
Gumned Film Sr 90

Estimate (mc/mi2)

0.11

.01

.03

.05

.04

.03

.03 


\section{TABLE}

New York City

Comparison of Pot Analyses and

Gummed Film Estimates of Strontium $90\left(\mathrm{mc} / \mathrm{mi}^{2}\right.$ )

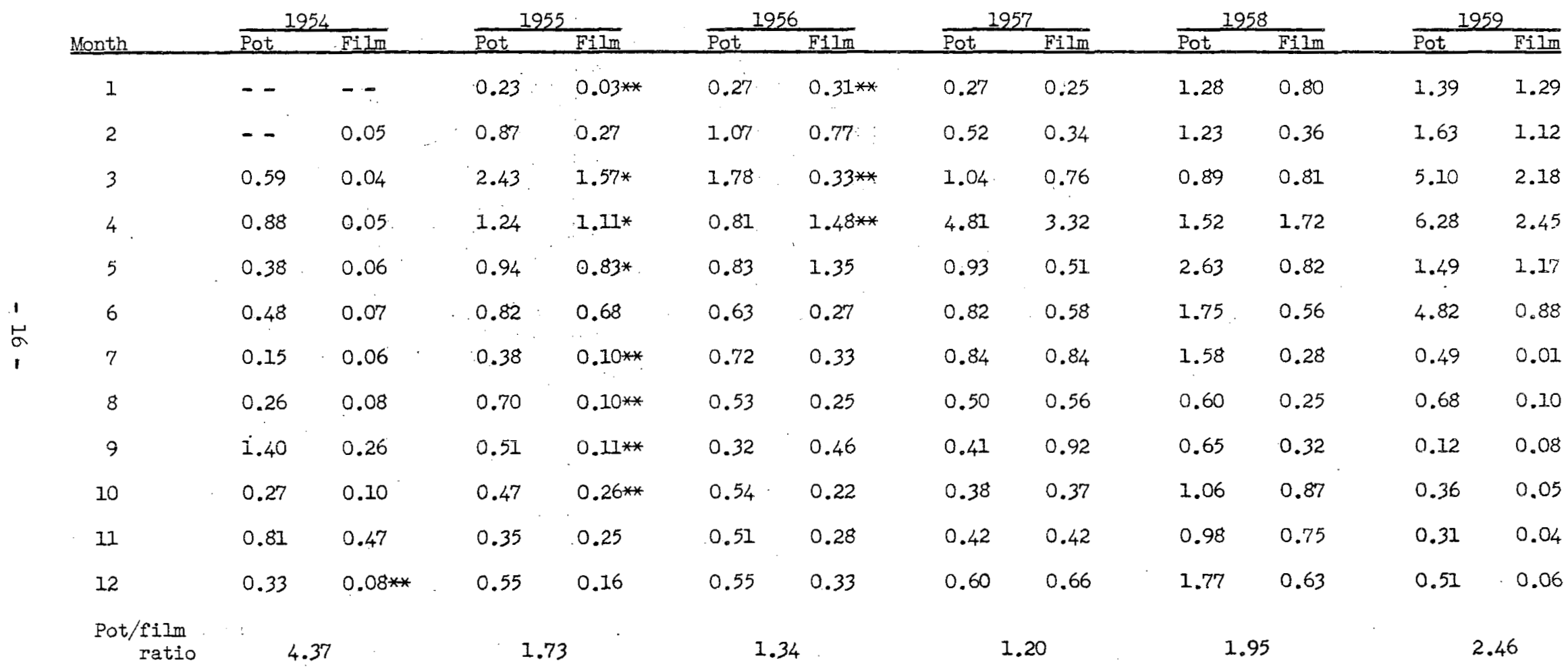

* Corrected for local fallout

* Monthly composite gumed film 
1. The gummed film results tend to be low when compared to pot analyses.

2. Results from composite samples are even lower with respect to the pot analyses than the summed daily values compared in adjoining months.

3. The highest daily pot/film ratios are found in 1954 , and the next highest are found in 1958 and 1959.

4. The daily pot/film ratios are generally good during 1955, 1956 and 1957.

Since the composite gummed film results were excessively low, a comparison was made for the months of February through June of 1955 where the samples from stations in the United States were run by both methods. This comparison showed the summed daily values to be 5 times as high as the composites on the average. This value may not be generally applicable and has not been used in preparing the summary tables for individual stations. The most probable causes are loss of sample in compositing and insufficient correction for self-absorption.

The monthly pot samples collected in New York City from 1954 through 1958 were analyzed for total $\beta$ activity as well as for $\operatorname{Sr}^{90}$. These total $\beta$ activity values have been treated by the present model, assuming the monthly collection to have been a single sample taken on the 15th of the month. (Pot samples before January 1957 were collected weekly and the effective date was taken as mid-week). This computation allows comparison of calculated and measured $\mathrm{Sr}^{90}$ from the same collector.

The data in Table 5 show a marked improvement over the comparison made in Table 4. This is surprising, since they are based on the same model and a factor (1.6) has been included to correct for gummed film efficiency. The difference is very marked for 1958, and an inspection of the 


\section{TABLE 5}

Comparison of $\mathrm{Sr}^{90}$ Values Measured in Pot Samples Collected In

New York City With Those Computed From Total B Activity in The Same Pot Samples Data Given in $\mathrm{mc} / \mathrm{mi}^{2}$

\begin{tabular}{|c|c|c|c|c|c|c|c|c|c|c|}
\hline \multirow[b]{2}{*}{ Month } & \multicolumn{2}{|c|}{1954} & \multicolumn{2}{|c|}{1955} & \multicolumn{2}{|c|}{1956} & \multicolumn{2}{|c|}{1957} & \multicolumn{2}{|c|}{1958} \\
\hline & Meas, & Comp & Meas. & Comp, & $\overline{\text { Meas }}$ & Comp. & Meas, & Comp. & Meas, & Comp \\
\hline I & - & - & 0.23 & 0.04 & 0.27 & .52 & 0.27 & - & 1.28 & 1.41 \\
\hline 2 & - & - & 0.87 & 0.27 & 1.07 & .04 & 0.52 & 0.37 & 1.23 & .99 \\
\hline 3 & 0.59 & - & 2.43 & 2.51 & 1.78 & 1.71 & 1.04 & 0.68 & 0.89 & 2.58 \\
\hline 4 & 0.88 & 0.26 & 1.24 & 1.50 & 0.81 & 1.88 & 4.81 & 1.38 & 1.52 & 4.50 \\
\hline 5 & 0.38 & 0.05 & 0.94 & - & 0.83 & 1.10 & 0.93 & 1.12 & 2.63 & 1.84 \\
\hline 6 & 0.48 & 0.03 & 0.82 & 0.74 & 0.63 & 0.13 & 0.82 & 0.94 & 1.75 & .72 \\
\hline 7 & 0.15 & 0.05 & 0.38 & 0.12 & 0.72 & 0.22 & 0.84 & 2.26 & 1.58 & 1.11 \\
\hline 8 & 0.26 & 0.07 & 0.70 & 0.33 & 0.53 & 0.58 & 0.50 & 2.83 & 0.60 & .35 \\
\hline 9 & 1.40 & 0.56 & 0.51 & 0.16 & 0.32 & 0.73 & 0.41 & 2.65 & 0.65 & .54 \\
\hline 10 & 0.27 & 0.15 & 0.47 & 0.31 & 0.54 & 0.26 & 0.38 & 0.51 & 1.06 & 2.67 \\
\hline 11 & 0.81 & 0.70 & 0.35 & 0.26 & 0.51 & 0.47 & 0.42 & 0.01 & 0.98 & .98 \\
\hline 12 & 0.33 & 0.21 & 0.55 & 0.14 & 0.55 & 0.64 & 0.60 & 0.92 & 1.77 & 1.91 \\
\hline Meas & & & & & & & & & & \\
\hline
\end{tabular}


total $\beta$ activity relation for routine pots and films was made. The total $\beta$ ratio of pot/filn for 1958 was found to be 2.25 rather than 1.6 .

The change in collection efficiency factor does not account for the difference between the computed values for pots and gummed film. It is not possible to evaluate the numerous other factors, such as the use of a single sampling date for a monthly collection; and no explanation is available.

Data for pot-gummed film comparisons at locations other than New York City are presented in Tables 6,7 and 8 . Since the earliest results are for 1957, there is no confirmation of the low estimates for 1954. As was the case for New York City, the 1957 comparison is better than that for 1958 and 1959.

It has been found from the HASI pot network data that little of the debris from the USSR tests in the fall of 1958 appeared far south of the equator. The model would be expected to strongly underestimate the $\mathrm{Sr} 90$ in the lower latitudes at the end of 1958 and in 1959. The data from Durban and Pretoria in Table 7 seem to confirm this.

Soil analyses provide only cumulative $\mathrm{Sr}^{90}$ deposition data, and not deposition rates. Since the inherent exror (not including sampling) in soil analysis is about $1.0 \%$ (12), the subtraction of $\mathrm{Sr}^{90}$ soil values to obtain deposition rate is not satisfactory. Thus, the only valid comparison of gummed film data with soils is for accumulated deposition.

There is a factor not accounted for in the comparisons made here. Soil values are reported as of the time of collection, while the summed gummed film values are as of their collection date. These sumned values are therefore too high by about 3\% per year, as no allowance has been made for Sr 90 decay after deposition. 


\section{TABLE 6}

Comparison of Pot Analyses and Gunmed Film Estimates of Strontium 90 Data given in $\mathrm{mc} / \mathrm{mi}^{2}$

\begin{tabular}{|c|c|c|c|c|c|c|c|c|c|c|c|c|}
\hline & & & go & Salt I & e city & Ios A & eles & & $\mathrm{Nag}$ & & $\mathrm{Hir}$ & \\
\hline & & Pot & FiIm & Pot & Film & Pot & Film & & Pot & Film & Pot & FiIm \\
\hline 1957 & 1 & 0.30 & .29 & 0.80 & .47 & 0.99 & .45 & & 1.01 & .93 & 0.29 & 32 \\
\hline & 2 & 0.27 & .35 & 0.83 & .59 & 0.76 & .27 & & 0.17 & .51 & 0.53 & .29 \\
\hline & 3 & 0.47 & .49 & 2.39 & 1.53 & $0.09 *$ & .37 & $:$ & 0.38 & .36 & 0.23 & .48 \\
\hline & 4 & 1.15 & .86 & 2.30 & 1.78 & 0.84 & .56 & & 1.98 & 1.16 & 1.12 & 1.33 \\
\hline & 5 & 0.27 & .67 & 0.81 & 1.49 & 0.24 & .20 & & 0.72 & .37 & 0.57 & .59 \\
\hline & 6 & 0.48 & .75 & 1.61 & 2.07 & 0.12 & .16 & & 0.27 & .19 & 0.49 & .23 \\
\hline & 7 & 1.57 & 1.34 & 0.94 & 2.40 & 0.92 & .39 & . & 1.07 & .85 & 0.82 & .50 \\
\hline & 8 & 0.75 & .65 & 1.28 & $2.06 * *$ & 0.05 & .08 & & 0.46 & .34 & 0.05 & .07 \\
\hline & 9 & 0.10 & .79 & $0.15 *$ & $1.88 * *$ & 0.04 & .14 & & 0.26 & .19 & 0.28 & .36 \\
\hline & 10 & 0.22 & .27 & 0.99 & 1.77 & 0.26 & .19 & & 0.21 & .28 & lost & .30 \\
\hline & 11 & 0.15 & .14 & 0.41 & .31 & 0.27 & .16 & & 0.19 & .09 & 0.14 & .09 \\
\hline - & .12 & -- & .49 & 0.64 & .43 & 0.20 & .46 & & 0.17 & .39 & 0.36 & .23 \\
\hline 1958 & 1 & $\delta_{0} 30$ & .16 & 0.70 & .39 & 0.44 & .23 & & 0.60 & .38 & 0.24 & .30 \\
\hline 0 & 2 & $\{0.30$ & .19 & 1.10 & .49 & 0.90 & .36 & & 0.55 & .32 & 0.25 & 24 \\
\hline - & 3 & lost & 1.24 & 1.47 & 2.26 & 1.30 & .93 & & 1.13 & .69 & 0.92 & .82 \\
\hline & 4 & 0.57 & 2.37 & 2.10 & 3.69 & 1.60 & .76 & & 2.52 & 1.22 & 3.37 & 1.83 \\
\hline & 5 & 0.79 & .96 & 1.30 & 1.69 & $0.05 *$ & .27 & & $1.75 *$ & .24 & 1.06 & .52 \\
\hline & 6 & 4.87 & 1.49 & 0.28 & .75 & 0.10 & .05 & & 0.88 & .49 & 0.64 & .35 \\
\hline & 7 & $0.09 *$ & .56 & $0.06 *$ & .59 & 0.16 & .06 & & 2.10 & 2.60 & 0.43 & 1.39 \\
\hline & 8 & $3.67 *$ & .44 & 0.71 & .57 & 0.08 & .15 & & 0.50 & .33 & $2.66 *$ & .29 \\
\hline & 9 & 0.33 & .21 & - & .24 & 0.08 & .08 & & 0.26 & .10 & 0.42 . & .20 \\
\hline & 10 & 0.76 & .35 & 0.10 & .38 & 0.15 & .14 & & 0.55 & .30 & 0.79 & .58 \\
\hline & 11 & 1.04 & .39 & 0.26 & .42 & 0.11 & .18 & & 0.61 & .22 & 0.71 & .27 \\
\hline & 12 & 0.42 & .25 & -- & .95 & 0.07 & .12 & & 2.10 & .72 & 0.57 & .40 \\
\hline 1959 & $I$ & 1.00 & 0.38 & 2.46 & 1.27 & 1.82 & 0.42 & & 3.00 & 1.10 & 1.81 & 0.85 \\
\hline & 2 & -- & 0.76 & 2.56 & 1.24 & 3.31 & 1.54 & & 2.14 & 1.09 & 2.45 & 1.27 \\
\hline & 3 & - & 3.41 & 4.56 & 1.21 & 0.10 & 0.42 & & 3.74 & 1.56 & 5.55 & 2.77 \\
\hline & 4 & 4.41. & 2.62 & 5.84 & 2.64 & 0.63 & 0.67 & & 7.60 & 2.53 & 6.52 & 2.39 \\
\hline & 5 & $4.19^{\circ}$ & 2.01 & $=-$ & 3.77 & 0.22 & 0.16 & & 3.02 & 0.93 & 0.40 & 0.99 \\
\hline & 6 & 0.58 & 0.52 & 2.40 & 1.44 & 0.09 & 0.08 & & 0.74 & .35 & 1.09 & 0.57 \\
\hline & 7 & 0.33 & 0.17 & 0.53 & 0.41 & 0.09 & 0.06 & & 1.73 & .64 & $\ldots$ & 0.51 \\
\hline & 8 & - & 0.08 & - & 0.25 & 0.05 & 0.06 & & 0.19 & .18 & -- & 0.16 \\
\hline & 9 & 0.90 & 0.08 & 0.69 & 0.20 & 0.03 & 0.05 & & 0.13 & .04 & 0.04 & 0.09 \\
\hline & 10 & $\ldots$ & 0.08 & 0.25 & 0.15 & 0.12 & 0.09 & & 0.06 & .08 & 0.27 & 0.09 \\
\hline & 11 & - - & 0.05 & 0.93 & 0.15 & 0.03 & 0.02 & & 0.09 & .06 & 0.13 & 0.10 \\
\hline & 12 & $\cdots$ & 0.05 & 0.26 & 0.10 & 0.39 & 0.11 & & 0.36 & .08 & 0.16 & 0.07 \\
\hline
\end{tabular}

* Pot Value Suspect

* Best Estimate, Not Formula 
TABLE?

Comparison of Pot Analyses and Gummed. Filn Estimates of Strontium 90 Data given in $\mathrm{Mc} / \mathrm{mi}^{2}$

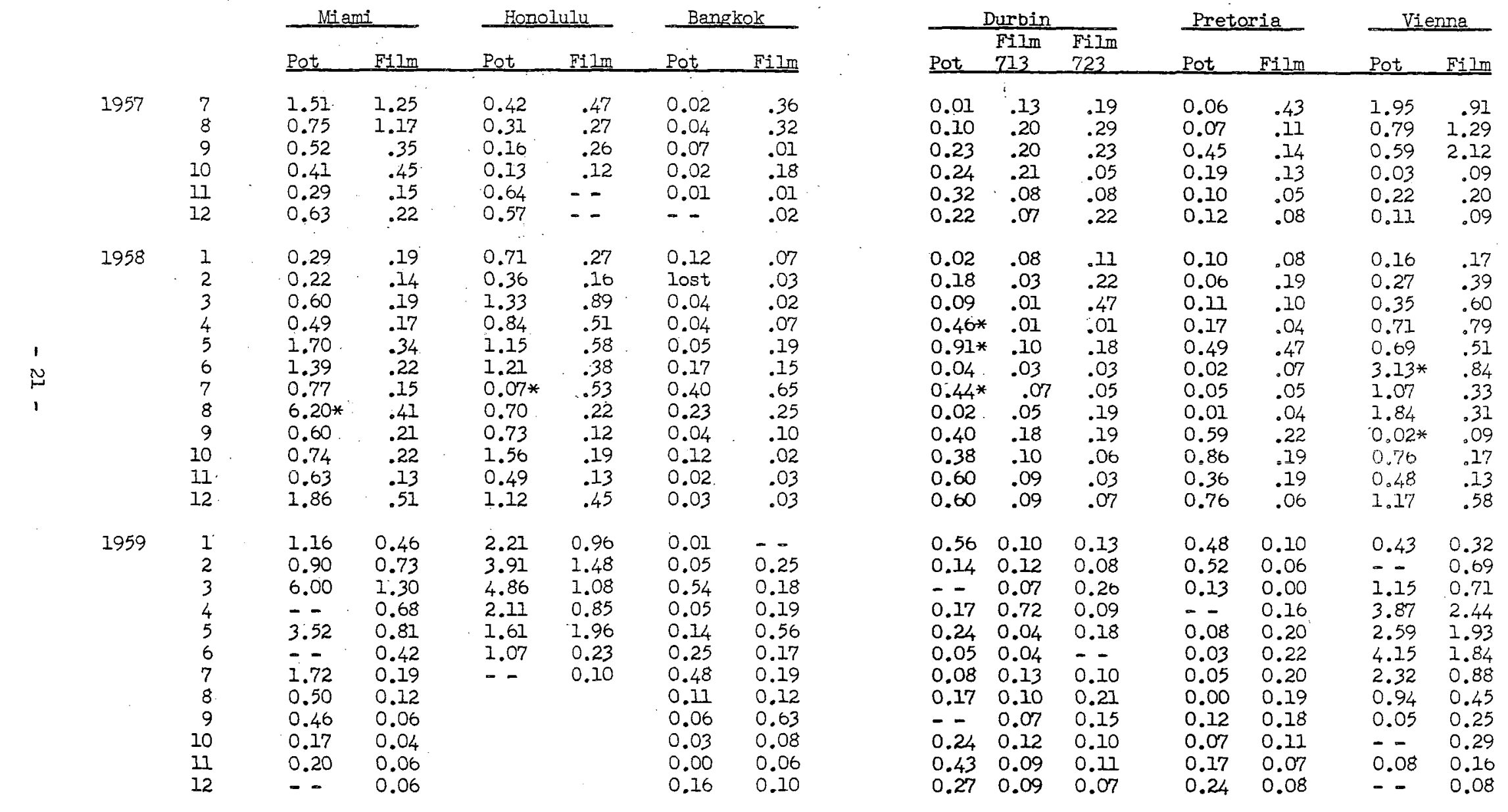

* Pot Value Suspect 


\section{TABLE 8}

Comparison of Pot Analyses and Gummed Film Estimates of Strontium 90 Data Given in $\mathrm{mc} / \mathrm{mi}^{2}$

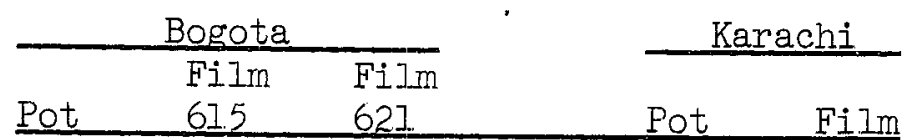

1958
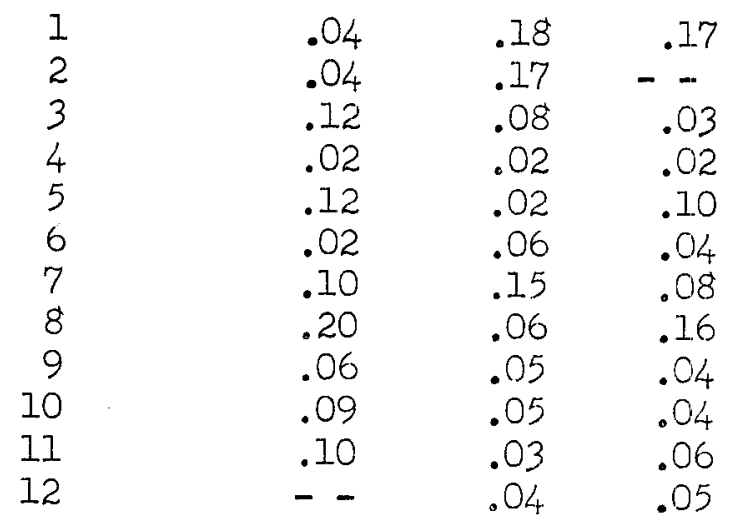

$\begin{array}{ccc}.04 & .17 & -. \\ .12 & .08 & .03\end{array}$

$\begin{array}{lll}.02 & .02 & .02\end{array}$

$\begin{array}{lll}.12 & .02 \quad .10\end{array}$

$\begin{array}{lll}.02 & .06 & .04\end{array}$

$\begin{array}{lll}.10 & .15 & .08\end{array}$

$.20 \quad .06 \quad .16$

$\begin{array}{lll}.06 & .05 & .04\end{array}$

$\begin{array}{lll}.09 & .05 & .04\end{array}$

$.10 \quad .03 \quad .06$

$\begin{array}{lll}- & .04 & .05\end{array}$

Pot Film

1959

1
2
3
4
5
6
7
8
9
10
11
12

$\begin{array}{ll}--\overline{0} & 0.13 \\ 0.06 & 0.08 \\ 0.07 & 0.02 \\ 0.25 & 0.09 \\ 0.02 & 0.07 \\ -- & 0.03 \\ -\overline{-} & 0.23 \\ 0.04 & \\ 0.02 & \\ -\overline{-} & \\ 0.33 & \\ -- & \end{array}$

0.06

0.09

0.06

$-$

$-$

$.02 \quad .25$

$.07 \quad .12$

$.13 \quad .19$

$.39 \quad .41$

$.07 \quad .30$

$.07 \quad .30$

$.15 \quad .21$

$.06 \quad .04$

$.06 \quad .04$

$.04 \quad .10$

lost .30

1959

0.12

0.06

$0.29 \quad 0.32$

0.39
$-\quad 0.39$

0.1 .5

$0.47 \quad 0.61$

- 0.40

- 0.44

$0.47 \quad 0.41$

$0.06 \quad 0.31$

0.07

0.07

$0.11 \quad 0.25$

- - 0.11

0.03

0.03

0.120 .14 
Table 9 presents comparisons of soil analyses and gummed film estimates for 17 sites in the United States. Once again, the model produces low results, particularly for the 1958 samplings.

It should be noted that the gummed Iilm estimate is high for Albuquerque, Grand Junction and Salt Lake City in 1955, 1956 and 1957. Even the 1958 estimates are high for the first two. This has been studied in some detail and is not considered to be the result of local fallout. The pot-film comparison for Salt Lake City in Table 6 shows high film estimates even when no local fallout is present, such as in the spring of 1958. A possible explanation is an increased collection efficiency for the gurnmed filn in dry areas. This may be enhanced by the collection of windblown material by the film. The windblown debris on the soil may later be blown off, while this is not possible with a gummed surface.

Table 10 presents the data available for other soil sampling sites. The results appear similar to those in previous tables, but the agreenent is better than for U.S. soil sites.

\section{Evaluation of Infinity Garma Dose Data}

There are no data at hand by which the gamma dose calculated from the gummed film measurements can be checked. While dosimetric measurements have been possible at a few locations following relatively high fallout from single bursts, no direct measurements are available on a continuous basis.

Gustafson ${ }^{(13)}$ has made some spectrometric measurements on soils and computed the gamma dose rate for the vicinity of Chicago. This is a laborious process and again, repeated observations over any extended period have not been reported. 
Comparison of Soil Analyses and Gummed Filn

Estiflutes of $\mathrm{Sr} \%$ at 17 Sites in the United States

Data given is $\mathrm{nc} / \mathrm{mi}^{2}$

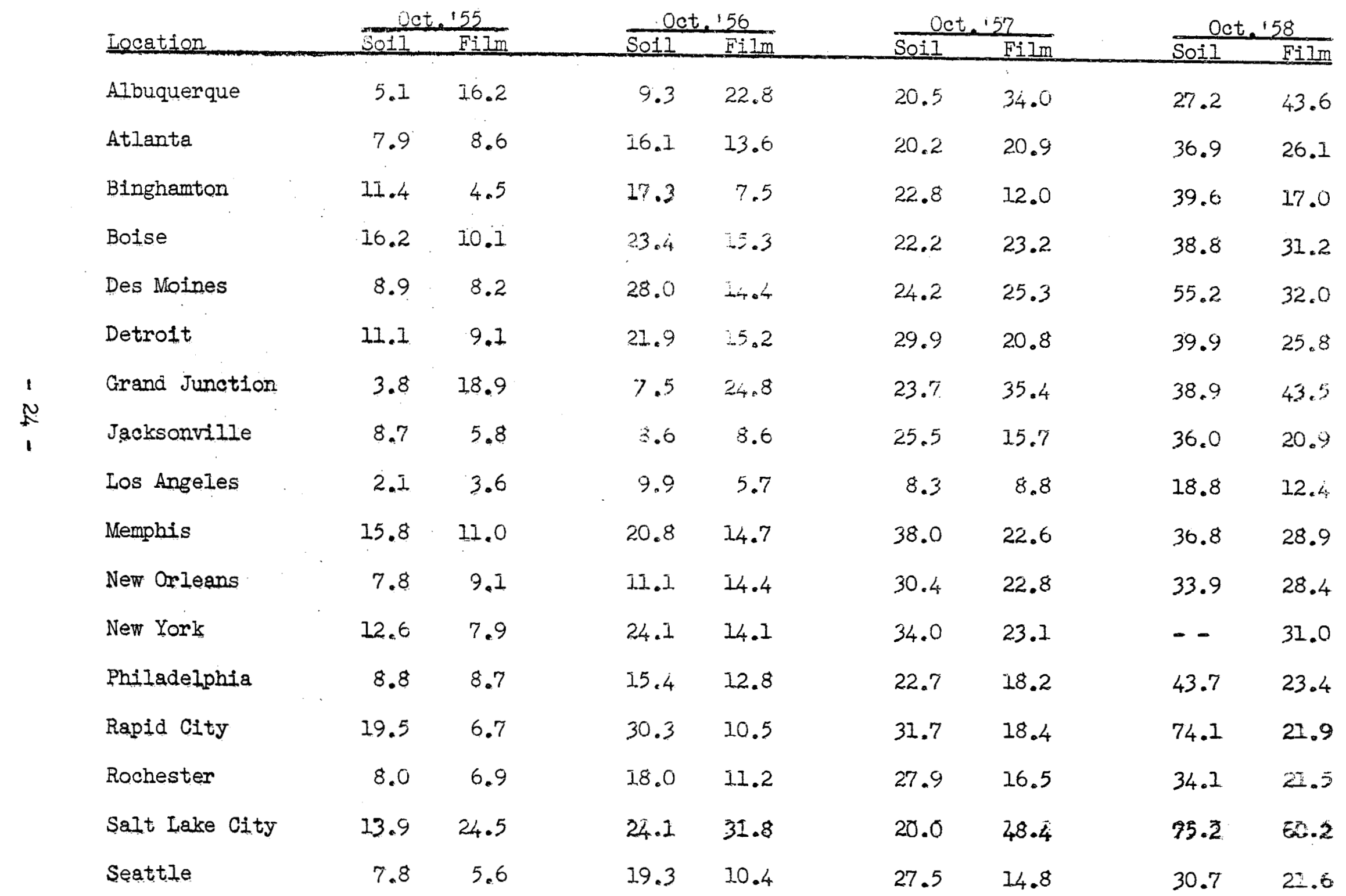


TABLE 10

Comparison of Soil Analyses and Gummed Film

Estimates of Strontium 90

Data given in $\mathrm{mc} / \mathrm{mi}^{2}$

\begin{tabular}{|c|c|c|c|c|c|c|}
\hline \multirow[b]{2}{*}{ Location } & \multicolumn{3}{|c|}{1956} & \multicolumn{3}{|c|}{1958} \\
\hline & $\begin{array}{l}\text { Sampling } \\
\text { Month }\end{array}$ & Soil & Film & $\begin{array}{c}\text { Sampling } \\
\text { Month }\end{array}$ & Soil & Film \\
\hline Fairbanks, Alaska & 8 & 4.2 & 7.0 & 6 & 12.5 & 12.6 \\
\hline Panama Canal Zone & 1 & $\begin{array}{l}4.9 \\
6.0\end{array}$ & 2.6 & 2 & $\begin{array}{l}7.1 \\
9.0\end{array}$ & .7 .4 \\
\hline $\begin{array}{l}\text { Lima, Peru } \\
\text { Buenos Aires, }\end{array}$ & 1 & 3.8 & 2.4 & 2 & 2.2 & 5.2 \\
\hline Argentina & 1 & 2.6 & 2.7 & 2 & 11.2 & 6.2 \\
\hline Bogota, Columbia & 1 & 2.5 & 2.5 & 2 & 4.7 & 6.0 \\
\hline Oslo, Norway & 8 & 11.6 & 4.9 & 9 & 28.6 & 12.7 \\
\hline $\begin{array}{l}\text { Durban, Union of } \\
\text { South Africa }\end{array}$ & 9 & $\begin{array}{l}4.7 \\
3.2\end{array}$ & 4.0 & 3 & 6.9 & 5.8 \\
\hline Hiroshima, Japan & 4 & 5.5 & 5.0 & & & \\
\hline $\begin{array}{l}\text { Nagasaki, Japan } \\
\text { Tokyo, Japan }\end{array}$ & 4 & 3.8 & 6.0 & & & \\
\hline $\begin{array}{l}\text { Mekyo, Japan } \\
\text { Melbourne, Australia } \\
\text { Welington, New }\end{array}$ & a & 3.0 & 3.7 & 2 & $\begin{array}{l}30.4 \\
24.6\end{array}$ & 17.9 \\
\hline $\begin{array}{r}\text { Zealand } \\
\text { Singapore }\end{array}$ & $\begin{array}{l}4 \\
4\end{array}$ & $\begin{array}{l}3.3 \\
2.6 \\
3.2\end{array}$ & $\begin{array}{l}2.5 \\
4.1\end{array}$ & $\begin{array}{l}3 \\
2\end{array}$ & $\begin{array}{l}9.7 \\
4.2\end{array}$ & $\begin{array}{l}4.8 \\
6.4\end{array}$ \\
\hline Sydney, Australia & 4 & 5.6 & 4.0 & & & \\
\hline Wake Island & 4 & 8.6 & 4.7 & 2 & 20.2 & 11.9 \\
\hline Canton Island & 11 & 3.5 & 2.8 & 2 & $\begin{array}{r}4.7 \\
11.3\end{array}$ & 5.1 \\
\hline Honolulu, Hawaii & 11 & 7.7 & 6.3 & 2 & 32.6 & 11.3 \\
\hline
\end{tabular}


The original computation of gamma dose from total beta activity on gummed film $(7)$ was developed to meet this lack of suitable dose estimates. Unfortunately, the gamma dose calculations are subject to the same errors in evaluating multiple bursts as the $\mathrm{Sr}^{90}$ estimates.

The gamma dose computed is the air dose to infinite time from uniform fallout on an infinite smooth plane. No reduction for shielding or weathering has been introduced, nor has there been included any build-up factor for scattering. The usual overall reduction factor is $10^{(14)}$ for converting to gonad dose. It might be noted here that the thirty year dose is only slightly less than the infinity dose.

There is a second possible estimate of gamma dose. If we assume that $\mathrm{Cs}^{137}$ is produced and deposited in a fixed ratio to $\mathrm{Sr}^{90}$, the dose from $\mathrm{Cs}^{137}$ can be computed. The calculations are based on the same assumptions as the total beta activity $(7)$. The modified formulas are:

$$
\text { Dose }=6.64 \times 10^{-10} \quad \sum N \text { in mrads }
$$

where,

$$
\begin{aligned}
& \text { Dose }=\mathrm{Cs}^{137} \text { infinity } \gamma \text { dose in millirads } \\
& \sum \mathrm{N}=\text { Total } \mathrm{Cs}^{137} \text { gamma disintegrations to infinity per sq. ft. }
\end{aligned}
$$

Accepting a half-life for $\mathrm{Cs}^{137}$ of 26.6 years and an initial $\beta$ dpm ratio for $\mathrm{Cs}^{137} / \mathrm{Sr}^{90}$ of 1.75 , there would be $2.8 \times 10^{9} \mathrm{Cs}^{137} \mathrm{\beta}$ disintegrations to infinity per sq. ft. for each $\mathrm{mc} / \mathrm{mi}^{2}$ of $\mathrm{Sr} 90$. This would give $2.3 \times 10^{9} \mathrm{Cs}^{137} \gamma$ disintegrations per sq. ft.

The final formula would be:

$$
\text { Dose }=1.5\left(\mathrm{mc} / \mathrm{mi}^{2} \mathrm{sr} 90\right) \text { in mrads }
$$

The estimate of gamma dose based on $\mathrm{Cs}^{137}$ will be extremely low for fallout occurring shortly after a test and will improve with age of the debris. 
Since the dose calculation for $\mathrm{Cs}^{137}$ requires only multiplication of the $\mathrm{Sr} 90 \mathrm{mc} / \mathrm{mi}^{2}$ by 1.5, this has not been done for the general tables. The comparison has been made in Table 11, however, for the dose deposited each year at New York City. The same data are shown in Table 12 for Salt Lake City, where a larger proportion of fresh fallout might be expected.

In Table 11, the ganma dose estimate from the pot $\mathrm{Sr}^{90}$ determinations is the highest, as would be expected from the New York City $\mathrm{Sr}^{90}$ comparison of pots and films. The Cs ${ }^{137}$ Film/Total $y$ Film ratios are the same for the two cities, indicating that the high yield tests overwhelm any effect from fresh fallout. 


\section{TABLE 11}

Comparison of Infinity Garme Dose Estimates

For New York City

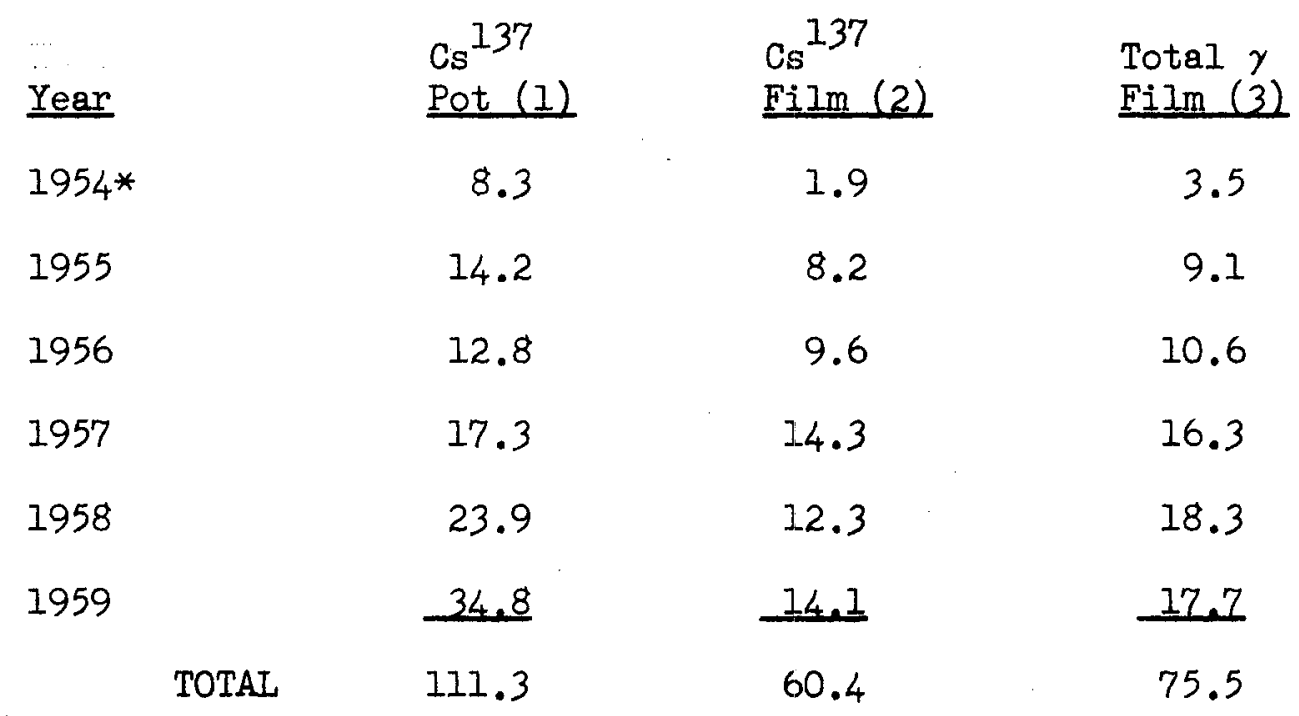

* Starting March 1

(1) $\mathrm{Sr}^{90}$ from pots $\times 1.5$

(2) $\mathrm{sr}^{90}$ from gummed film $\times 1.5$

(3) Calculated by formula including all fission products 


\section{TABLE 12}

Comparison of Infinity Garnma Dose Estimates for Salt Lake City

Year

1954

1955

1956

1957

1958

1959

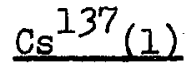

3.9

20.3

9.9

25.2

18.6

19.2

97.1
Total y (2)

8.9

24.5

12.3

29.0

25.8

23.2

123.7

(1) $\mathrm{Sr}^{90}$ from gummed film $\times 1.5$

(2) Calculated by formula including all fission products 


\section{SUMMARY}

While the gummed film network fulfilled its original purpose of delineating fallout patterns, this report has been devoted to the extension of the $\beta$ activity measurements to the estimation of $\mathrm{sr}^{90}$ deposition and infinity gamma dose. The computations have been based on a simple model, but yield $\mathrm{Sr}^{90}$ estimates that are generally within a factor of two from measured values. It would be possible to improve the gummed film estimates by adopting a more sophisticated model, but the added cost would not be justified. The present results have required much greater effort than was expected and have appeared more slowly than was hoped.

The gummed film $\mathrm{Sr}^{90}$ estimates are lower in most cases than the measured values at nearby sampling sites. The deviation is probably a combination of many effects:

1. The estimate of $\mathrm{Sr}^{90}$ yield may be low, particularly for fractionated debris, where long range fallout is enriched in this nuclide.

2. The gunmed film efficiency is variable and is probably lowest during heavy rainfalls when a large fraction of the $\mathrm{Sr} 90$ is deposited.

3. The gummed film efficiency continues to decrease in the absence of $\frac{1}{3}$ arge scale tests, for the percentage of long-lived $\mathrm{Sr} 90$ and $\mathrm{Cs}^{137}$ increases and their retention is poor.

4. The present model is poor during periods of repeated high yield tests, as there is no delay"included for arrival time from either the troposphere or stratosphene. The debris is thus always considered to be fresher than the actual fallout.

The results presented in this report have some value in the absence of direct measurements. While it is not possible to evaluate all situations, the data may be used for geographic interpolation between stations where $\mathrm{Sr}^{90}$ results are available. It is also possible to make reasonable estimates for 
stations having only gummed film sampling by the use of a regional Pot/Gummed

Film factor. As an example, the following table was developed from the northern hemisphere stations where pot data were collected.

Average Pot/Gummed Film Ratios for the Northern Hemisphere

Month

Jan.

Feb.

Mar.

Apr.

May

June

July

Aug.

Sept.

Oct.

Nov.

Dec.

Mean Ratio

1957

1.4

1.3

1.2

1.4

.9

1.0

1.1

.7

.5

.7

1.3

1.1 $\underline{1958}$

1.6

1.8

1.1

1.0

1.6

2.4

1.2

2.1

2.3

2.0

2.0

2.5

1.8
1959

2.1

2.0

2.8

2.1

1.6

2.5

2.6

1.9

1.5

2.1

2.4

3.5

These average ratio factors can still yield only an approximation and probably hold only for the northern hemisphere.

There is some justification for applying the same factors to the infinity gamma dose data as well. Most of the errors can be divided into two classes, poor collection of fission products and those involved in the model computation. Poor collection would affect the $\mathrm{Sr}^{90}$ and $\gamma$ dose values equally. The computation model is equivalent except for the factors of $\% \mathrm{Sr}^{90}$, and of $\gamma$ disintegrations per $\beta$ dpm. The first is approximately a $T^{+1.2}$ function, while the second is approximately $\mathrm{T}^{+0.73}$ for the first year and $\mathrm{T}^{+1.2}$ thereafter. Thus, the average Pot/Film ratio may be a reasonable correction factor. There has been no attempt in this report to depict the geographical distribution of $\mathrm{Sr}^{90}$. The data do not warrant this, as soil and pot analyses are more reliable for this purpose. 


\section{Sampling}

The sampling medium is a rubber base cement spread on a $13^{\prime \prime} \times 13^{\prime \prime}$ cellulose acetate backing and covered with glassine paper for protection. For sampling, the glassine is removed and the film attached to a cadmiumplated steel frame having a one foot square opening. The frame is held three feet above the ground on a stand. Each paper is exposed for twentyfour hours, the sampling being started at 1230 Greenwich Civil Time at each station. At the end of the sampling period fresh films are put in place on spare frames and exchanged with the exposed films. The exposed films are stripped off and folded with the gummed sides together into a size suitable for mailing. Most sampling stations collect duplicate samples, and the two samples plus a field data card giving the location, date of sampling, and weather information are forwarded to the Health and Safety Laboratory.

\section{Sample Preparation}

An important point in processing is maintaining identification of the sample from receipt to the reporting of the analytical results. For this purpose a six digit serial number is assigned to each sample. The first three digits indicate the day of analysis on a counting house calendar basis, while the last three digits indicate the number of the sample run during that day. When the sample envelope is opened, a prenumbered label is placed on the data card and the gummed films are placed in steel cans for ashing. These ashing cans are used as crucibles and are discarded after a single use. For convenience and speed of operation they are held in groups of ten in a stainless steel rack and the rack number and position within the rack are the actual indication of sample number for each can. 
The samples are ashed at 500 to $550^{\circ} \mathrm{C}$ in electrically-heated muffle furnaces which hold individual racks. The ashing process is accelerated by feeding in about one liter of oxygen per minute to each furnace and requires slightly under one hour for completion. After cooling, the ash is transferred to a 1-1/4" diameter plastic counting planchet. Each planchet has been labeled with a gummed label which is a duplicate of the one used on the data card. This maintains the proper continuity and agreement of serial numbers. The planchets are then sealed between two layers of vinyl tape in groups of eighty. The sealing is done with a high frequency heater which makes a ring seal around each planchet. The samples are spaced about $9 "$ on centers and the resultant tape can be readily handled in an automatic counter.

Each tape contains four blanks consisting of unexposed papers run through the process and four counter standards. Counter background is determined from the blanks and counter efficiency and reliability is determined from the standards.

Counting

The samples are analyzed on automatic beta counters with Geiger tube detectors. A reel of eighty planchets is loaded into the counter and the first sample is positioned above the Geiger tube which is mounted face up. The sample is separated from the tube face by a single thickness of vinyl tape and is held in position by a fixed stop which engages the side of the planchet. The counts are registered on a four Dekatron scaler. Simultaneously, 600 pulses per minute are applied to this scaler and are recorded on a duplicate parallel scaler. When 10,000 count plus time pulses are recorded on the first scaler, the number of time pulses is punched on paper tape by a Data Transfer and Recording (DTR) system developed by the Instrumentation 
Division. At the same time, the serial number corresponding to the sample, machine identification and other data are punched into the tape. The sample stop is disengaged by a solenoid and the next sample is pulled into place and counted. In that way, with eight units operating, 640 samples, blanks and standards can be counted in a twenty-four hour period.

The basic standardization of the counters is set up on potassium chloride. The $\mathrm{K}^{40}$ isotope, present in consistent amounts in all potassium compounds, gives a disintegration rate of 29.0 dps per gram of potassium or 912 dpm per gram of potassium chloride. It has been found that the average weight of ash from fallout samples is about $100 \mathrm{mg}$. While the counter standards used are compressed pellets of four grams to reduce the counting time, their effective disintegration rate is referred to $100 \mathrm{mg}$ of potassium chloride. Data Handling

All calculations are performed on IBM equipment. The field data cards are turned over to punch operators where location, date of sampling, serial number, and weather data are punched. The paper strips from the DTR unit are automatically transferred to separate IBM cards. The two sets of data are then combined on the basis of sample serial number, and the single cards are ready for computation. Figure 1 is a flow sheet of the system indicating all of the steps carried out in producing the final reports. 


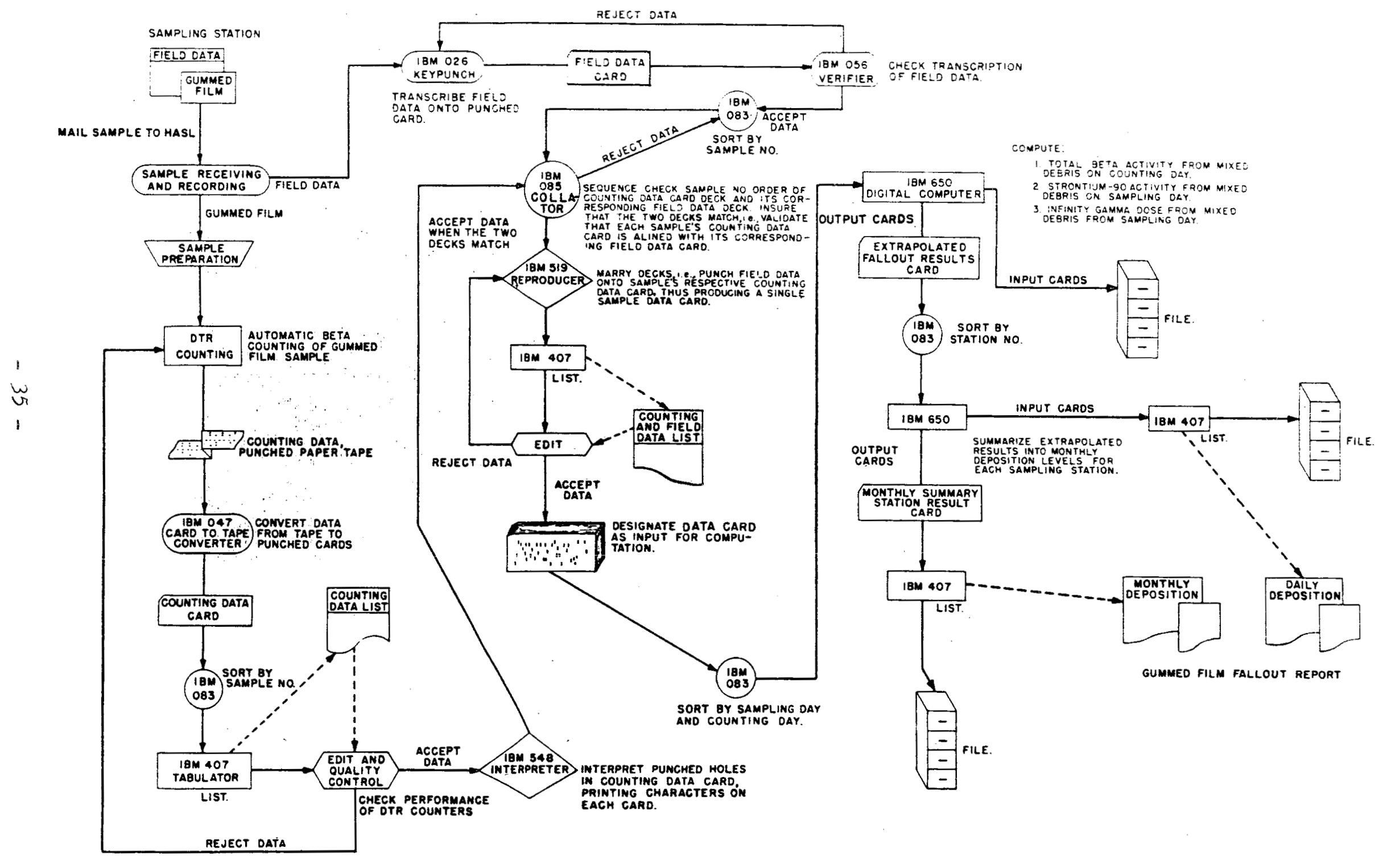




\section{BIBLIOGRAPHY}

(1) Webb, J.H., "Fogging of Photographic Film by Radioactive Contaminants in Cardboard Packaging Materials," Phys. Rev, 76 , 375 (1949)

(2) "Radioactive Debris from Operations Buster and Jangle," U. S. Atomic Energy Commission Report NYO-1576, January 28, 1957, Declassified with deletions, April, 1959

(3) Eisenbud, M. and Harley, J. H., "Radioactive Dust from Nuclear Detonations," Science, 117, 141-147 (1953)

(4) Eisenbud, M. and Harley, J. H., "Radioactive Fallout in the United States;" Science, 121, 677-680 (1955)

(5) Eisenbud, M. and Harley, J.H., "Radioactive Fallout through September 1955," Science, 124, 251-255 (1956)

(6) Hunter, H. F. and Ballou, N. E., "Simultaneous Slow Neutron Fission of U235 Atoms," Nucleonics 2, No. 11 C-2 (1951)

(7) Hallden, N. A. and Harley, J. H., "Method of Calculating Infinity Gamma Dose," U. S. Atomic Energy Commission Report NYO-4859, April 15, 1957

(8) Eisenbud, M. and Harley, J. H., "Long-Term Fallout," Science, 128, 399402 (1958)

(9) The Effects of Atomic Weapons, U. S. Government Printing Office, Washington, D. C. (1950)

(10) Marinelli, I. D., Brinckerhoff, R. F. and Hine, G. J., "Average Energy of Beta-Rays Emitted by Radioactive Isotopes," Rev, Mod. Phys., 19, 25-28 (1947)

(11) Welford, G. A. and Collins, W. R., "An evaluation of Existing Fallout Collection Methods," Science, 131, 1791-1793 (1960)

(12) Alexander, I. T., et al, "Strontium 90 on the Earth's Surface," reported in U. S. Atomic Energy Commission Report HASL-84, April 1, 1960

(13) Gustafson, P. F., Argonne National Laboratory, Reported in the Atomic Energy Commission Quarterly Statement on Fallout, April 1, 1960

(14) "Report of the United Nations Scientific Committee on the Effects of Atomic Radiation," p. 101, New York (1958) 


\section{SECTION II}

\section{MONTHLY SUMMARIES FOR INDIVIDUAL STATIONS}

Because of the change in emphasis for different test series and because of differences between Nevada Tests and Pacific and Foreign Tests, the network has been subject to considerable change over the years. The majority of the data presented are for sampling stations which have had a continuous history since the end of 1952 when the first world-wide network was established. Some stations entering the network later, particularly those set up in cooperation with the U. N. Scientific Committee on the Effects of Atomic Radiation, are also included.

Stations having data for only limited periods are not listed. These would include:

1. Special stations operating for short periods of time around the two United States test sites.

2. Certain stations operating in cooperation with the U. S. Public Health Service.

3. Other stations not forwarding samples on a continuous basis.

A map of the network is given in Figure 2. The stations operating directly with HASL (Table 13) are distinguished from those operating through the United Nations Scientific Committee (Table 14). Table 15 is made up of the summary tables for all stations in order of station number.

In all of the summary tables, composite samples are marked with a single asterisk (*). Where geographical interpolation was required to fill out a table, the values are marked with a double asterisk (**). 
The effect of fresh fallout from single bursts has been taken into account in some cases. For these months, a figure also appears in the column headed "Formula". This figure represents the actual value calculated by the model, while the figure under "Best Estimate" represents the value obtained after allowing for fresh fallout. In all other cases, the "Best Estimate" figure is also the calculated value.

In the final totals, the pre-1954 data are based on single burst computations described in the "Historical" section of this report. 


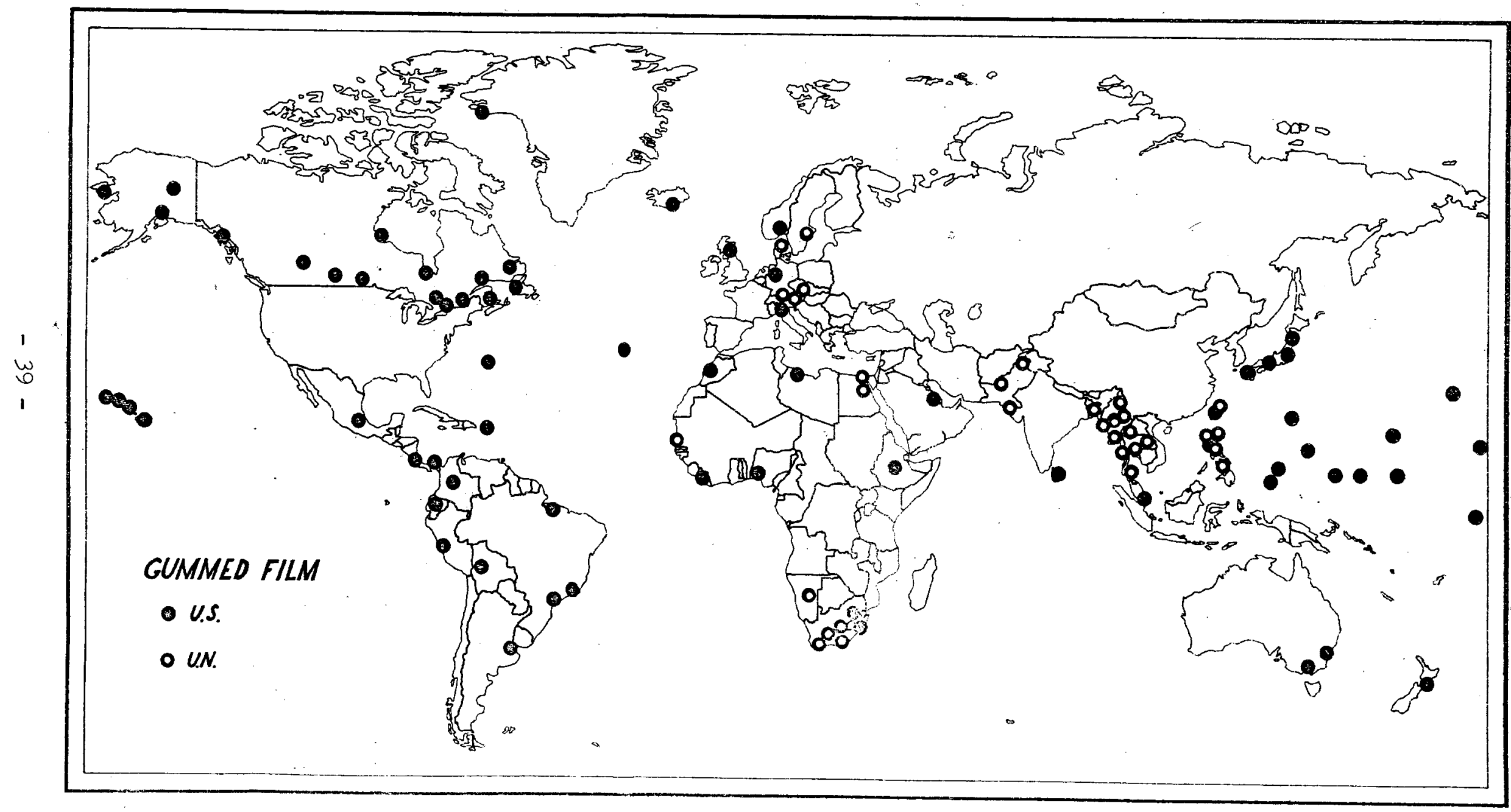

Figure 2 
TABLE 13

NUMERICAL INDEX OF AEC

WORID-WIDE NETWORK STATIONS

101 Detroit, Michigan

102 Louisville, Kentucky

103 Knoxville, Tennessee

105 Memphis, Tennessee

108 Atlanta, Georgia

115 Philadelphia, Pennsylvania

116 Pittsburgh, Pennsylvania

117 New York City, LaGuardia

118 Binghamton, New York

122 Rochester, New York

127 New Haven, Connecticut

132 Jacksonville, Florida

133 Miami, Florida

134 Washington, D. C.

137 Cleveland, Ohio

138 Cape Hatteras, North Carolina

139 Concord, New Hampshire

141 Boston, Massachusetts

204 Corpus Christi, Texas

206 Dallas, Texas

209 Wichita, Kansas

211 Scotisbluff, Nebraska

212 Rapid City, South Dakote

216 Minneapolis, Minnesota

219 Des Moines, Iowa

221 St. Louis, Missouri

222 Chicago, Illinois

225 New Orleans, Louisiana

304 Boise, Idaho

309 Billings, Montana

310 Salt Lake City, Utah

314 Tucson, Arizona

321 Grand Junction, Colorado

323. Albuquerque, New Mexico

326 Las Vegas, Nevada

401 Seattle, Washington

404 Medford, Oregon

407 San Francisco, California

410 Los Angeles, California.

501 Anchorage, Alaska

502 North Bay, Ontario, Canada

503 Moosoonee, Ontario, Canada

504 Moncton, New Brunswick, Canada

505 Montreal, Quebec, Canada

507 Seven Islands, Quebec, Canada

508 Winnipeg, Manitoba, Caneda 
TABLE 13 (Cont)

509 Churchill, Manitoba, Canada

510 Regina, Saskatchewan, Canada

511 Edmonton, Alberta, Canada

515 Deep River, Ontario, Canada

516 Goose Bay, Newfoundland, Canada

517 Stephenville, Newfoundland, Canada

518 Thule, Greenland

519 Keflavik, Iceland

522 Nome, Alaska

523. Fairbanks, Alaska

524 Juneau, Alaska

525 San Juan, Puerto Rico

601 Panama Canal Zone, Albrook AFB

602 Bermuda, Kindley AFB

603 Lima, Peru

604 San Jose, Costa Rica

605 Lagens, Azores

606 Buenos Aires, Argentina

608 Sao Paulo, Brazil

611 Belem, Brazil

612 La Paz, Bolivia

613 Quito, Ecuador

614: Mexico City, Mexico

615 Bogota, Colombia

701 Prestwick, Scotland

702 Rhein Main, Germany

703 Pretoria, Union of South Africa

705 Dhahran, Saudi Arabia

706 Sidi Slimane, Morocco

708 Oslo, Norway

710 Tripoli, Libya, Wheelus AFB

711 Lagos, Nigeria

713 Durban, Union of So. Afr1ca, Unfversity

714 Monrovia, Liberia

715 Milan, Italy

717 Addis Ababa, Ethiopia

723 Durban, Union of So. Africa, Natal Airport

$\$ 01$ Hiroshima, Japan

802 Nagasaki, Japan

804 Taipei, Taiwan, First Station

805 Tokyo, Japan, Haneda AFB

806 Misawa, Japan

810 Melbourne, Australia

811 Wellington, New Zealand

813 Colombo, Ceylon

814 Singapore

815 Sydney, Australia

901 Guam, Marianas

903 Iwo Jima, Volcano Islands 
TABLE 13 (Cont)

904 Manila, Phillipines, Clarke AFB

905 Johnson Island

906 French Frigate Shoals, Hawail

907 Midway Island

908 Wake Island

909 Canton, Phoenix Islands

910 Ponape, Marshall Islands

911 Truk, Caroline Islands

912 Yap, Palau Islands

913 Koror, Palau Islands

914 Iinue, Hawai1

915 Honolulu, Hawai1

916 Hilo, Hawail

922 Kwajalein, Marshall Islands 
NUMERICAL INDEX OF STATIONS OPERATED COOPERATIVELY BY AEC AND GOVERNMENT OF INDICATED COUNTRY

621 Bogota, Colombia, Tibaitata

722 Vienna, Austria, Wien Hohe Warte

724 Salzburg, Austria

725 Innsbruck, Austria

726 Krippenstein, Austria

727 Klagenfurt, Austria

728 Port Elizabeth, Union of So. Africa

729 Cape Town, Union of So. Africa

730 Beaufort, Union of So. Africa

731 Bloemfontein, Union of So. Africa

732 Windhoek, Union of So. Africa

735 Stockholm, Sweden

737 Dakar, French West Africa

821 Quetta, Pakistan

822 Peshawar, Pakistan

823 Karachi, Pakistan

824 Dacca, Pakistan

825 Bangkok, Thailand

826 Chiengmai, Thailand

827 Ubol, Thailand

828 Songlshla, Thailand

829 Taipei, Teiwan, Second Station

830 Rangoon, Burma

831 Taipei, Taiwan, Third Station

832 Akyab, Burma

833 Lashio, Burma

834 Moulmein, Burna

835 Prome, Burma

836 Mergui, Burma

837 Mandalay, Burma

838 Myitkyina, Burma 
TABLE 15

Monthly Summaries of Estimated $\mathrm{Sr}^{90}$ Deposition

and Infinity Gamma Dose Through Deoember 1959

\author{
Stations are listed in order of \\ station numbers as given in \\ Tables 13 and 14 .
}




\section{ESTIMATE OF Sr 90 DEPOSITION FROM GUMNED FILM}

STATION

LOOATION_DETROIT, MICHIGAN

$1254 \quad 1955 \quad 1256 \quad 1957$

Eormula Best Este Formula Best Este Fornula Best Este Formula Best Est.

Jan.

0.04

$0.09 *$

$0.21 *$

0.28

Feb.

.06

.49

.52

.27

March

.03

2.53

.35*

.41

Apr11

.13

7.57

2.24

.60**

.88

May

.05

.61

2.25

.61

June

.10

$.22 *$

JuIy

.05

$.11 *$

Aug.

.04

$.13 *$

Sept.

.19

.12*

.30

.54

.19

.67

oct.

.20

Nov.

.34

.11.**

Dec.

TOTAL

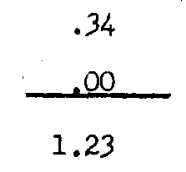

.22

.43

.24

.60

.89

$\frac{.15}{7.02}$

.32

.28

.14

.28

.82

$\frac{0.64}{5.85}$

2958

1959

Formula Best Est. Formule Best Est.

$\begin{array}{lcc}\text { Jen. } & 0.25 & 0.48 \\ \text { Feb. } & .16 & .83 \\ \text { March } & .24 & 1.82 \\ \text { Apr11 } & 1.08 & 2.64 \\ \text { Mey } & .64 & 1.78 \\ \text { June } & .57 & .47 \\ \text { July } & .33 & .17 \\ \text { Aug. } & .21 & .11 \\ \text { Sept. } & .23 & .07 \\ \text { Oct. } & .41 & .07 \\ \text { Nov. } & .53 & .03 \\ \text { Dec. } & .36 & .06 \\ \text { TOTAL } & 5.01 & 8.53\end{array}$


1254 Eormula Best Esto

Jan.

Feb.

March

April

May

June

Jury

Aug.

Sept.

oct.

Nov.

Dec.

TOTAL
0.1

.1

.2

.7

.2

.4

.1

.1

.5

.5

.8

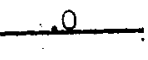

3.7
1955

Formula Best Est.

$0.2 *$

1.0

4.9

14.1

4.2

1.1

$.4^{*}$

$.2 *$

$.2 *$

$.2 *$

$.2 * *$

.4

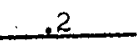

13.2
1956 Eormula Best Est.

$0.3 *$

.8

$.5 *$

$.9 * *$

3.4

.6

.4

1.0

1.3

.6

.5

.5

10.8
2057.

Formula Best Est.

0.5

.4

.7

1.6

1.0

1.0

1.1

.4

1.4

.6

.2

1.5

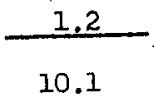

1958 1959

Formula Best Est. Formula Best Est.

$\begin{array}{lcc}\text { Jen. } & 0.4 & 1.0 \\ \text { Feb. } & .3 & 1.7 \\ \text { March } & .5 & 3.4 \\ \text { Apri1 } & 1.8 & 4.8 \\ \text { May } & 1.3 & 3.1 \\ \text { June } & 1.1 & .8 \\ \text { July } & .9 & .3 \\ \text { Aug. } & .5 & .2 \\ \text { Sept. } & .5 & .1 \\ \text { Oct. } & 1.4 & .1 \\ \text { Nov. } & 1.6 & .1 \\ \text { Dec. } & -.8 & .2 \\ \text { TOTAL } & 11.1 & 16.7\end{array}$


STATION \# 102

LOCATION_ LOUISVILLE, KENTUCKY

Cormule Best Est.

Eormula Best Est.

1956

Eormula Best Est.

Formula Best Est.

Jan.

0.04

$0.06 *$

$0.18 *$

0.41

Feb.

.07

.45

March

.15

2.46

1.16

Apr1I

May

June

JuIy

.08

.82

.04

$10.22 \quad 4.57$

.06

Aug.

.14

Sept.

.05

Sept.

.13

oct.

.22

Nov.

.32

Dee.

.03

$.48 *$

$.26 *$

$.09 *$

$.11 *$

$.23 *$

.17

TOTAL

1.33

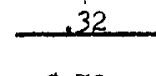

8.72

.73

.55

$.65 *$

.68

$.11 *$

.80

.65

.65

.29

1.26

1.03

.54

2.62

1.51

.47

.99

.69

.78

.24

.21.

.13

.13

23

37

4.91

8.07

1958

1952

Formula Best Est. Eormula Best Est.

Jan.

Feb.

March

Apr11

May

June

July

Aug.

Sept.

oct.

Nov.

Dec.

TOTAL.
0.41

.38

.82

1.72

1.02

.71

.88

.23

.22

.18

.51

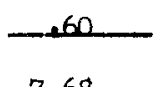

7.68 i. 27

1.32

2.56

2.97

1.40

.63

.18

.12

.10

.08

.08

.07

10.72

\begin{tabular}{rr} 
Pre-1954 & 1.44 \\
1954 & 1.33 \\
1955 & 8.72 \\
1956 & 4.91 \\
1957 & 8.07 \\
1958 & 7.68 \\
1959 & 10.72 \\
\hline Total & 42.9
\end{tabular}


STATION \# 102 Formula Besit Est.

Jan.

0.0

Feb.

March

Apri1

May

June

JuIy

Aug.

Sept.

Oet.

Nov.

Dec.

TOTAL
1955 Eormula Best Est. $0.1 *$

.1

.8

.4

.2

.2

.4

.1

.3

.5

.7

.0

3.7

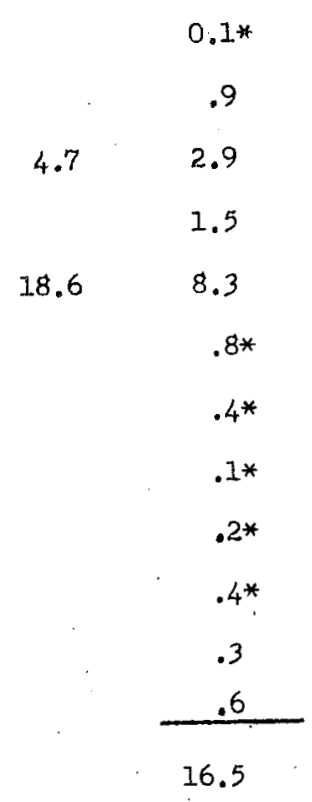

LOCATIO

LOUISVIILE, KENNTUCKY
1956 Pornils Begt Est.

$0.3 *$

1.2

$1.0 *$

$.2 *$

1.1

.6

1.3

1.0

1.5

.4

.3

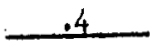

9.3
2957

Formula Best Esta

0.7

.9

1.1

1.4

1.1

$2.3 \quad 1.8$

$4.3 \quad 2.5$

1.6

1.3

.4

.3

.7

13.8

Formula Best Est.

tormule Best Est.

Jan;

0.7

2.6

Feb.

.6

March

1.6

2.6

Apr 11

3.0

May

2.0

June

1.3

July

2.3

Aug.

Sept.

oct.

Nov.

Dec.

TOTAL

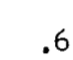

4.9

5.4

2.4

. 1.0

.3

.2

.1

.1

.1

1.5

1.4

16.1

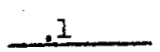

\begin{tabular}{rr} 
Pre-1954 & 6.3 \\
1954 & 3.7 \\
1955 & 16.5 \\
1956 & 9.3 \\
1957 & 13.8 \\
1958 & 16.1 \\
1959 & 19.8 \\
\hline Tota1 & 86.0
\end{tabular}

19.8 
ESTIMATE OF Sr90 DEPOSITION FROM GUMMDD FILM

STATION \#_IO3

LOCATION KNOXVILLE, TENNESSEE

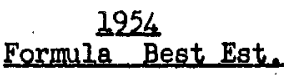

Jan.

Feb.

March

April

May

June

July

Aug.

Sept.

Oct.

Nov.

Dec.

TOTAL

$$
0.02
$$

.07

.10

.06

.10

.05

.07

.10

.06

.10

.38

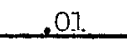

1.12
1955

Formula Best Est.

$0.07 *$

.23

1.55

.80

9.58

3.10

$.15 *$

$.08 *$

$.06 *$

$.38 *$

$.27 *$

.08

.34

7.01
1956

Eocmula Best Este

$0.19 *$

.61

$.22 *$

$.28 *$

.37

.20

.75

.21

.27

.23

.22

.29

3.85
1957

Formule Best Est.

0.36

.42

.47

.47

.39

.68

$1.74 \quad 1.28$

.58

1.73

.86

.30

.55

8.09

Formula Begt Est. Formul Best Est.

Jan.

Feb.

March

Apr11

May

June

July

Aug.

Sept.

Oct.

Nov.

Dec.

TOTAL,
0.41

.40

.58

1.45

.60

.31

.40

.17

.16

.34

.46

$-62$

5.90
0.83

$1: 31$

$1: 43$

1.79

1.00

.40

.12

.13

.06

.08

.06

.05

7.26 
STATION \#. 103<smiles>[14CH3]</smiles>

Eormula Best Eato

Jan。

0.0

Feb.

Maroh

April

May

June

JuIy

Aug 。

Sept.

Oat.

Nov.

Deo.

TOTAL
.1

.6

.3

.5

.2

.2

.3

.1

.2

.9

.0

3.4
2055

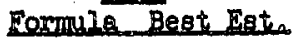

$0.1 *$

.5

2.9

1.5

17.3

5.6

$.3 *$

$.1 *$

$.1 *$

$.6 *$

$.4 *$

.1

.6

12.8
1956

Docmile Best Esto

$0.3^{*}$

1.0

$.3 *$

$.4 *$

.6

.4

1.7

.5

.6

.4

.4

.6

7.2
1957

Eocmula Best Est.

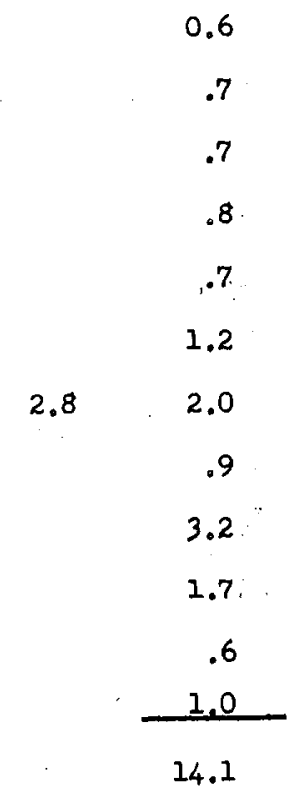

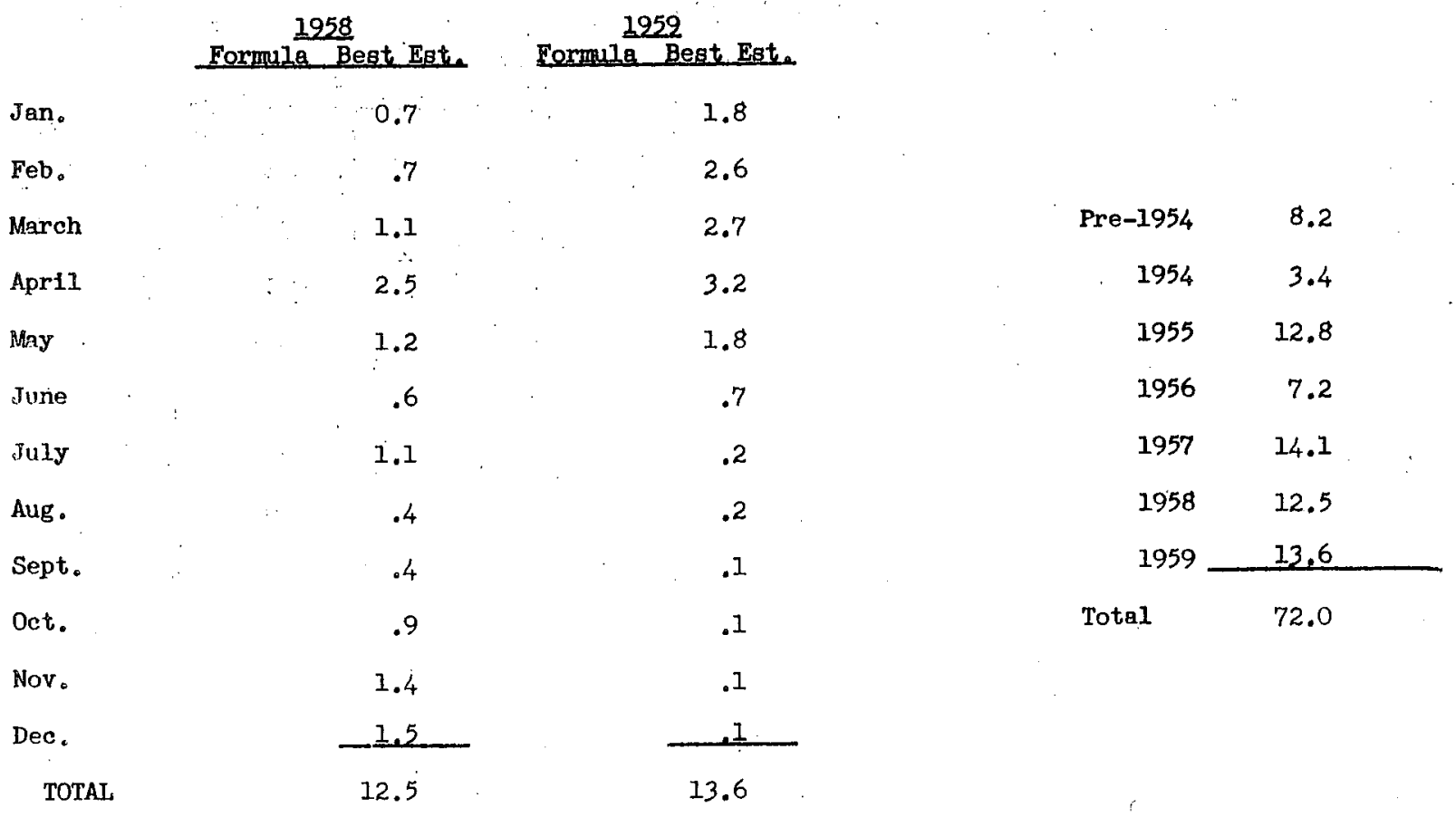


1955

1956

Eormula Best Est.

$0.30 *$

Jen。

0.04

Feb.

Maroh

April

May

June

July

Aug .

Sept.

oct.

Nov.

Deo.

TOTAL
Eormule Best Est.

$0.05 *$

.39

1.27

0.76

1.61

10.01

2.53

$.40 *$

$.10 *$

$.1 .1 *$

$.04 *$

$.10 *$

.07

i7

6.33
.46

$.30 *$

$.48 *$

.83

.16

.27

.21

.28

.14

.12

23

3.79
0.36

1957

Fermulo Best Est.

.31

.85

.92

.68

0.72

1.25

1.09

0.63

.74

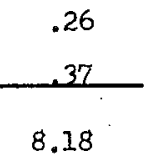

Formula Best Est. Formula Best Est.

Jan.

Feb.

March

Apr11

May

June

July

Aug .

Sept.

Oct.

Nov.

Dec.

TOTAL
0.32

.32

1.12

1.58

.60

.44

.60

.26

.18

.22

.44

$-13$

6.51
1.16

.89

1.97

1.53

.66

.55

.19

.13

.05

.05

.09

.08

7.35

\begin{tabular}{rl} 
Pre-1954 & 3.83 \\
1954 & 1.09 \\
1955 & 6.33 \\
1956 & 3.79 \\
1957 & 8.18 \\
1958 & 6.51 \\
1959 & 7.35 \\
\hline Total & 37.1
\end{tabular}


STATION \#_ 105

LOCATION MEMEHLS, TENNESSEE

1254

Formula Best Est.

Jan.

Feb 。

March

Apr11

May

June

July

Aug .

Sept.

Oct.

Nov.

Dec.

TOTAL

\begin{tabular}{l}
0.0 \\
.1 \\
.7 \\
.5 \\
1.1 \\
.3 \\
.2 \\
.1 \\
.1 \\
.2 \\
.4 \\
.1 \\
\hline 3.8
\end{tabular}

$0.1 *$

.8

2.4

18.1

3.0

4.6

$.7 *$

$.2 *$

$.2 *$

$.1 *$

.2*

.1

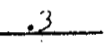

11.9
1956 Formula Best Est.

$0.5 *$

.7

$.5 *$

$.7 *$

1.3

.3

.6

.5

.6

.3

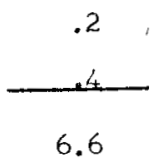

1957

Formula Best Est.

0.6

.5

1.4

1.7

]. 2

1.3

$3.1 \quad 2.1$

1.8

$1.6 \quad 1.0$

1.6

.5

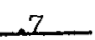

14.4

1958

1252

Formula Best Est.

Jan.

Formula Best Est.

Feb.

March

Apr11

May

June

July

Aug .

Sept.

oet.

Nov.

Dec.

TOTAI.

0.5

.6

2.2

2.7

1.2

.8

1.6

6

2

7

8

.7

.4

.7

1.3

$-1.0$

$13 . ?$
2.5

1.8

3.7

2.8

1.2

.9

.3

.2

.1

.1

\begin{tabular}{rr} 
Pre-1954 & 23.3 \\
1954 & 3.8 \\
1955 & 11.9 \\
1956 & 6.6 \\
1957 & 1.4 .4 \\
1958 & 13.7 \\
1959 & 13.8 \\
\hline
\end{tabular}

Total

88.0

.1

.1

13.8 
1954

1255

Cormula Best Est.

Apr11

May

June

July

Aug .

Sept.

oot.

Nov.

Deo.

TOTAL
0.04

.07

.15

.08

.12

.13

.13

.05

.03

.04

.34

.02

1.20
$0.05 *$

.11

1.10

.94

1956

Formula Best Est.

$0.27 *$

.71

$.16 *$

0.86

.45

.21

.72

.29

.23

.24

.12

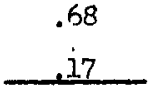

7.24

$.42 *$

$.10 *$

68

18

4.44
1957

Eoxmula Best Est.

0.35

.43

.76

.22

.27

.68

$1.04 \quad 0.62$

.47

33

Formula Best Est. Formula Begt Est.

$\begin{array}{lcc}\text { Jan. } & 0.38 & 0.61 \\ \text { Feb. } & .14 & 1.18 \\ \text { March } & .60 & 2.22 \\ \text { April } & 1.47 & 1.64 \\ \text { May } & .49 & .70 \\ \text { June } & .35 & .36 \\ \text { July } & .45 & .25 \\ \text { Aug. } & .26 & .14 \\ \text { Sept. } & .19 & .09 \\ \text { Oct. } & .11 & .07 \\ \text { Nov. } & .30 & .07 \\ \text { Dec. } & -.271 & .09 \\ \text { Torsi. } & 5.75 & 7.42\end{array}$

\begin{tabular}{rl} 
Pre-1954 & 1.04 \\
1954 & 1.20 \\
1955 & 7.24 \\
1956 & 4.44 \\
1957 & 7.47 \\
1958 & 5.75 \\
1959 & 7.42 \\
\hline Total & 34.6
\end{tabular}




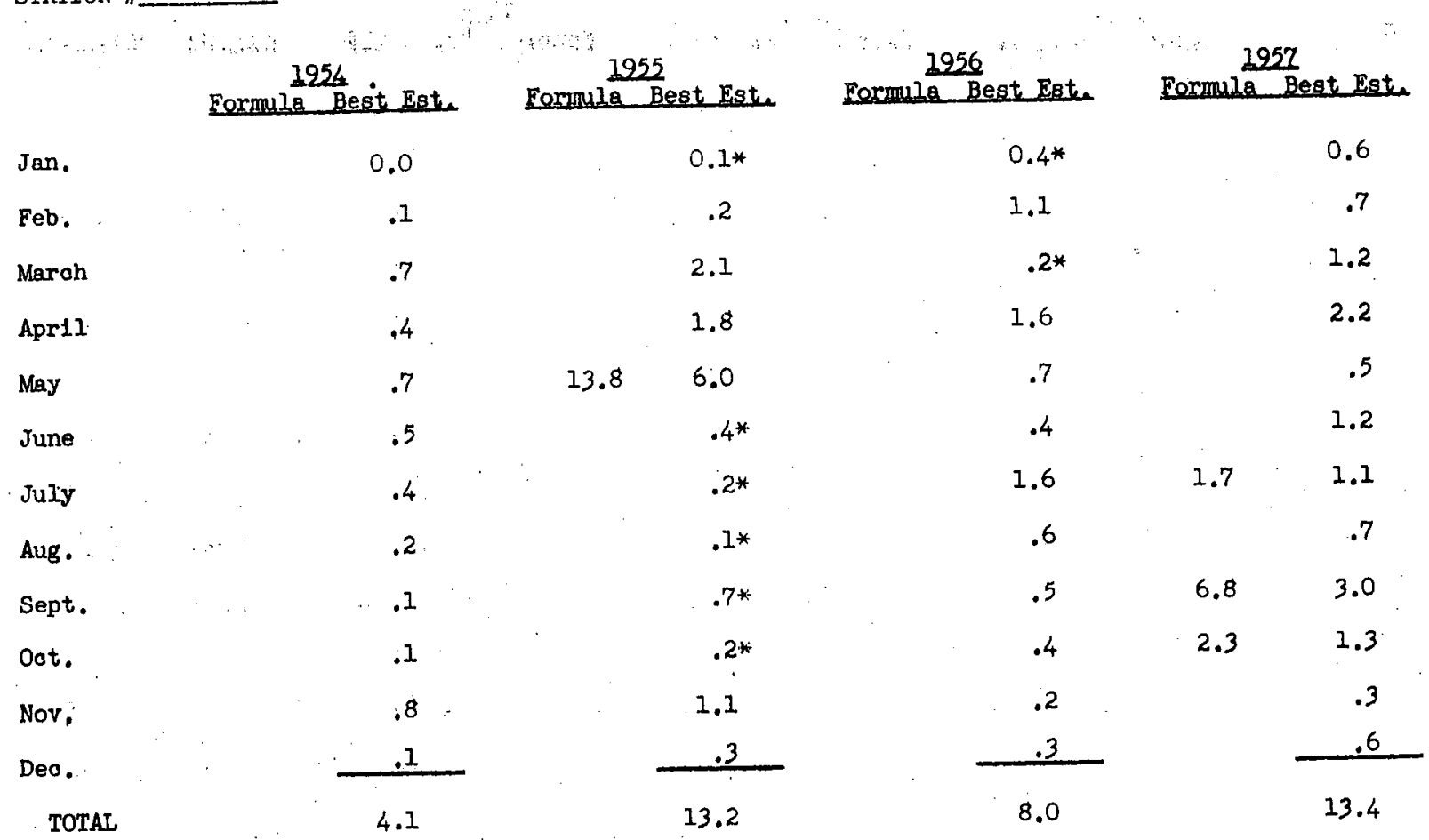

1958

Formula Best Est.

1959

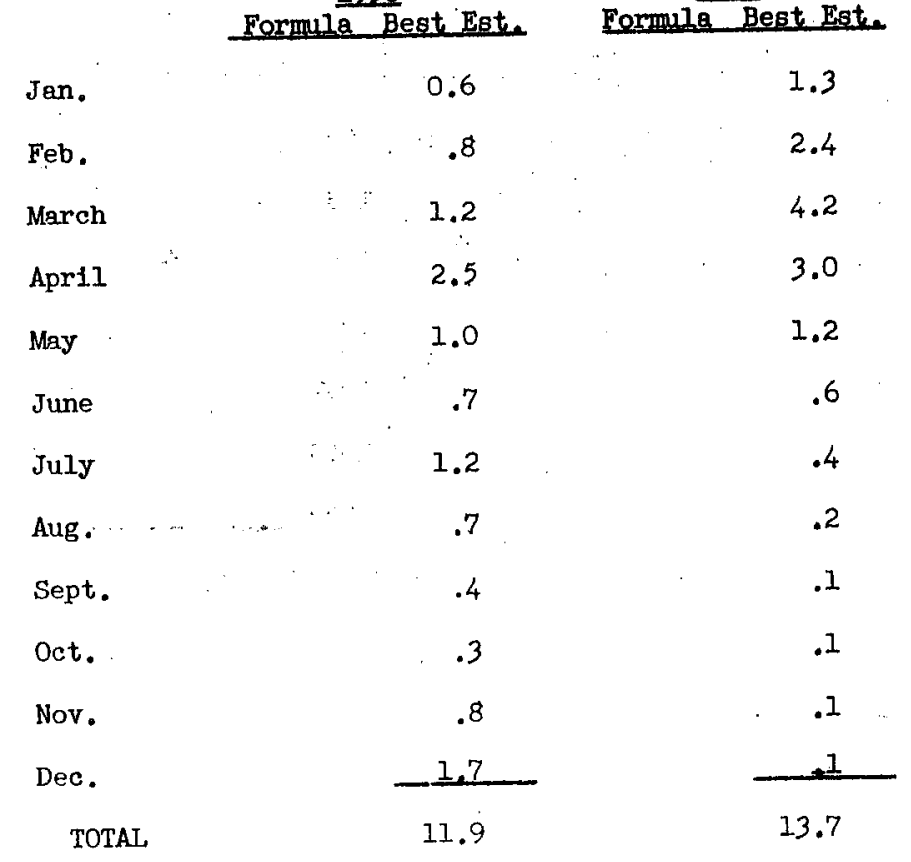

\begin{tabular}{rr} 
Pre-1954 & 3.7 \\
1954 & 4.1 \\
1955 & 13.2 \\
1956 & 8.0 \\
1957 & 13.4 \\
1958 & 11.9 \\
1959 & 13.7 \\
\hline Total & 68.0
\end{tabular}


STATION \# 115

$$
\text { 1954 }
$$

Eormula Best Est.

Jan.

0.04

Feb.

Maroh

Apri1

May

June

July

Aug.

Sept.

Oot.

Nov.

Deo.

TOTAL
.05

.04

.02

.03

.05

.06

.06

.19

.08

.27

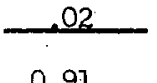

0.91
1955

Formule Best Est.

$0.05 *$

.29

1.35

$1.49 \quad .87$

2.93

1.85

.87

$.15 *$

$.16 *$

$.12 *$

$.15 *$

.08

$\frac{33}{6.27}$

LOCATION PHILADELPHIA, PENNSYLVANIA

1956

Formula Best Est.

$0.08 *$

.55

$.39 *$

$.28 *$

1.03

.23

.31

.14

.43

.19

.22

.25

4.10
0.20

Formula Best Est.

.23

.85

.87

.31

.49

.56

.14

.93

.38

.17

.34

5.47

2958

Eormula Best Est.

Jan.

0.44

2959

Feb.

.31

March

.65

1.4

Apr11

May

1.35

.52

June

.35

July

.28

Aug .

.24

Sept.

.17

oct.

.39

Nov.

.44

Dec.

TOTAL

.63

5.77

1.42

1.89

.37

.40

.10

.12

.06

.05

.05

.05

6.29

\begin{tabular}{rl} 
Pre-1954 & 1.94 \\
1954 & 0.91 \\
1955 & 6.27 \\
1956 & 4.10 \\
1957 & 5.47 \\
1958 & 5.77 \\
1959 & 6.29 \\
\hline Total & 30.8
\end{tabular}

Total 
STATION \# 115

1954 Formula Besit Est.

Jan.

0.0

Feb.

March

Apr11

May

June

July

Aug:

Sept.

Oct.

Nov.

Dec.

TOTAL

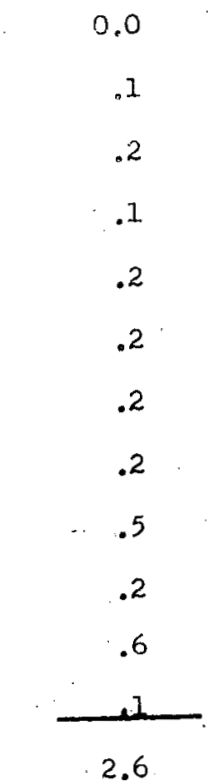

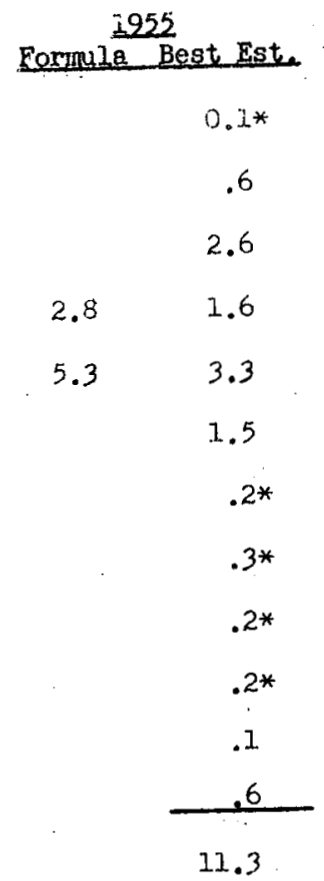

LOCATION PHIIADEIPHIA, PENNSYLVANIA

1957

$\begin{array}{cc}\frac{1956}{2957} \text { Best Est. } & 0.4 \\ 0.1^{*} & .4 \\ .9 & 1.4 \\ .6 * & 1.5 \\ .4^{*} & .5 \\ 1.5 & .9 \\ .5 & .9 \\ .7 & .2 \\ .3 & 1.5 \\ .9 & .9 \\ .4 & .3 \\ .4 & -9.5 \\ .5 & 9.5 \\ 7.2 & \end{array}$

1959

Formula Best Est. Formula Best Est.

Jan.

Feb.

March

Apri1

May

June

July

Aug .

Sept.

oct.

Nov.

Dec.

TOTAL
0.7

.5

1.3

2.3

1.0

.7

.7

.6

.4

1.5

1. 3

$-35$

12.5
1.9

1.8

2.7

3.4

.6

.7

.2

.2

.1

.1

.1

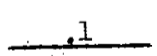

11.9

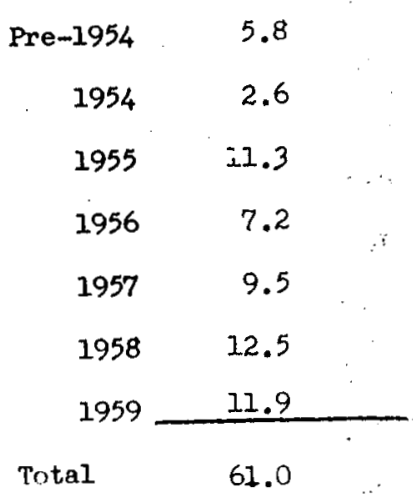

Total

61.0 
STATION \# 116

WOCATTON_PITTSBURGH, PENNSYUVANTA

1954

1955

2956

1952

Eormula Best Est.

Jen.

Feb。

Maroh

April

May,

June

July

Aug .

Sept.

oot.

Nov.

Dec.

TOTAL
0.05

.06

.07

.05

.04

.09

.08

.08

.17

.15

.25

.05

1.14
Formula Best Est.

$0.04 *$

.24

1.36

1.19

.81

$4.19 \quad 1.70$

.67

$.12 *$

.09*

$.06 *$

$.13 *$

.11

.23

5.56
Eormuli Best Est.

$0.35 *$

1.17

$.37 *$

$1.43 *$

1.38

.34

.33

.36

.58

.21

.28

29

7.09
Formule Best Est.

0.30

.42

.47

1.26

.33

.87

$1.27 \quad 0.88$

.33

.54

.39

.15

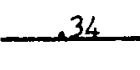

1958

Formula Best Este Formule Best Eat.

Jañ.

0.40

0.80

Feb.

.28

1.35

March

.23

1.44

Apr11

2.10

2.72

May

.82

1.45

June.

.64

July

.45

.60

.18

Aug .

.20

.10

Sept.

.22

.06

Oct.

.23

.10

\begin{tabular}{rl} 
Pre-1954 & 1.28 \\
1954 & 1.14 \\
1955 & 5.56 \\
1956 & 7.09 \\
1957 & 6.28 \\
1958 & 6.28 \\
1959 & 8.96 \\
\hline Tota1 & 36.6
\end{tabular}

Nov.

$.43 * *$

.09

Dec.

TOTAL

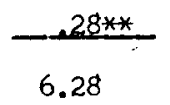

$\frac{.07}{8.96}$ 
STATTON \# 116

\section{Formula Best Est.}

0.1

Jan.

Feb.

March

April

May

June

July

Aug .

Sept.

oot.

Nov.

Deo.

TOTAL
.1

.4

$: 2$

2

.4

.2

.2

.4

.4

.6

.1

3.3
1955

Formula Best Est.

$0.1 *$

:5

2.6

$2.1 \quad 1.5$

7.5

3.0

1.2

$.2 *$

$.1 *$

$.1 *$

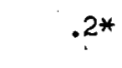

.2

.4

10.1
2956

Eormula Best Est.

$0.6 *$

1.9

$.6 *$

2.1*

2.1

.7

.8

.8

1.3

.4

.5

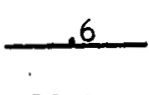

12.4
2957

Formule Best Este

0.5

.7

.8

2.1

.6

1.6

$2.1 \quad 2.4$

.5

.9

.8

.3

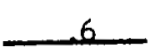

10.8

Formula Best Est. Formin Best Est. 1259

\begin{tabular}{lcc} 
Jan. & 0.7 & 1.7 \\
Feb. & .5 & 2.7 \\
March & .5 & 2.7 \\
April & 3.6 & 4.9 \\
May & 1.6 & 2.5 \\
June & 1.2 & 1.0 \\
July & 1.2 & .3 \\
Aug. & .5 & .2 \\
Sept.. & .5 & .1 \\
Oct. & .9 & .1 \\
Nov. & $.7 * *$ & .1 \\
Dec. & $-.5 * *$ & .1 \\
\multicolumn{1}{c}{ TotAL } & 12.4 & 16.4
\end{tabular}

Pre-1954 $\quad 3.9$

$1954 \quad 3.3$

$1955 \quad 10.1$

$1956^{\circ} \quad 12.4$

$1957 \quad 10.8$

$1958 \quad 12.4$

$1959+164$

Total

69.0 
1954

Eormula Beot Est.

Jan.

Feb.

March

Apr11

May

June

July

Aug .

Sept.

oct.

Nov.

Deo.

TOTAL
0.04

.05

.04

.05

.06

.07

.06

.08

.25

.10

.47

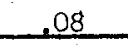

1.35
2955

Eormule Best Est.

$0.03 *$

.27

$3.51 \quad 1.57$

1.651 .11

$1.85 \quad .83$

.68

$.10 *$

$.10 *$

$.11 *$

$.26 *$

.25

.16

5.47
1956 Eormule Best Est.

$0.31 *$

.77

$.33 *$

$1.48 *$

1.35

.27

.33

.25

.46

.22

.28

.33

6.38
2957

Formula Best Est.

0.25

.34

.76

3.32

.51

.58

.84

.56

.92

.37

.42

.66

9.53

1958

1959

Formula Best Este Formula Best Est.

Jan.

0.80

i. 29

Feb.

.36

1.12

March

.81

2.18

April

1.72

May

June

.82

2.45

1.17

.56

July

.28

Aug .

.25

Sept.

.32

oct.

.87

Nov

.75

Dec.

TOTAL

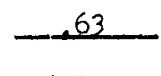

8.17

.88

.01

.10

.08

.05

\begin{tabular}{rr} 
Pre-1954 & 1.48 \\
1954 & 1.35 \\
1955 & 5.47 \\
1956 & 6.38 \\
1957 & 9.53 \\
1958 & 8.17 \\
1959 & 9.43 \\
\hline & 41.8
\end{tabular}

.04

.06

9.43 


\section{ESTIMATE OF INEINITY $\gamma$ DOSE FROM GMMMD FILM}

STATION \# 117

LOCATION NEW YORK CTTY

1954 Eormula Besit Eat.

1952

1956

2952

0.0

$\operatorname{Jan}_{0}$

$0.1 *$ Conmula Best Egt:

Formila Best Eat.

Feb。

.1

.5

Maroh

.2

6.8

2.1

3.1

2.0

Apr11

.2

3.3

1.5

May

.3

.3

1.2

June

.2

Aug.

.2

Sept.

.7

Oct.

.2

Nov.

1.1

Dec.

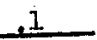

TOTAL

3.6

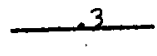

3.1

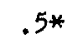

.4

.6

1.2

5.5

.9

1.0

1.4

.9

1.4

1.0

.8

.9
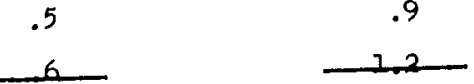

10.6

16.3.

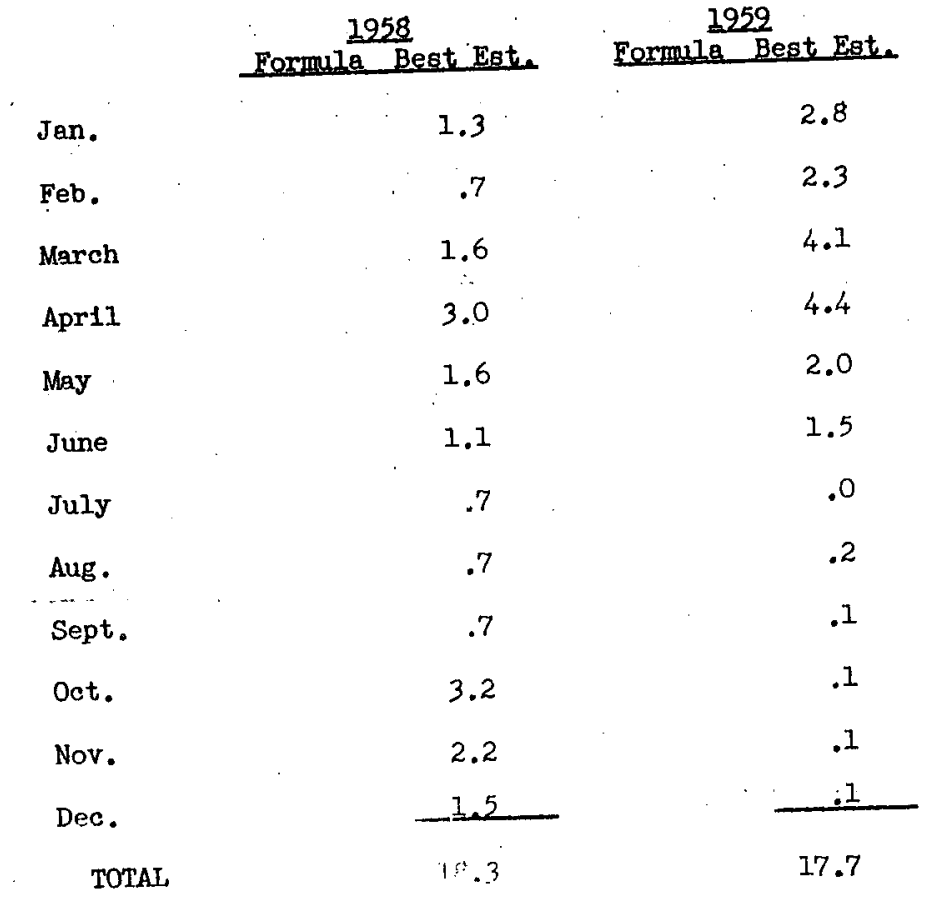


1954 Formula Best Este.

Jan.

Feb .

.

Marcin

Apr11

May

June

JuIy

Aug .

Sept.

oct.

Nov.

Deeo.

TOTAL
0.04

1955 Formula Best Est.

$0.01 *$

.05

.02

.04

.03

.06

.09

.09

.26

.09

.30

.01

1.08
.12

.59

.91

.77

$.17 *$

$.09 *$

$.05 *$

$.04 *$

$.12 *$

.21

.10

3.18
1956 Eormule Best Est.

$0.05 *$

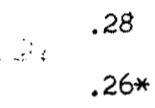

$.27 *$

.72

.40

.18

.25

.30

.07

.08

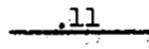

2.87
1957

Formula Best Est.

0.11

.12

.27

.62

.45

.48

.72

.52

0.66

.35

.11

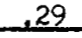

4.70

1958 1959

Formis Be Best Est. Formula Best Est.

$\begin{array}{lcc}\text { Jan. } & 0.39 * * & 0.28 \\ \text { Feb. } & .28 * * & .41 \\ \text { March } & .25 & 1.01 \\ \text { April } & .85 & 1.24 \\ \text { May } & .91 & .63 \\ \text { June } & .61 & 1.00 \\ \text { July } & .37 & .20 \\ \text { Aug. } & .23 & .12 \\ \text { Sept. } & .22 & .07 \\ \text { Oct. } & .47 & .06 \\ \text { Nov. } & .55 & .07 \\ \text { Dec. } & .33 & .07 \\ \text { TotaL } & 5.46 & 5.16\end{array}$

\begin{tabular}{rr} 
Pre-1954 & 0.59 \\
1954 & 1.08 \\
1955 & 3.18 \\
1956 & 2.87 \\
1957 & 4.70 \\
1958 & 5.46 \\
1959 & 5.16 \\
\hline Total & 23.0
\end{tabular}


STATION \# 118

LOCATION BINGHAMTON, NEW YORK

1254 Formla Best Est.

Jan.

Feb.

March

Apr11

May

June

July

Aug .

Sept.

oot.

Nov.

Dec.

TOTAL
0.0

.1

.1

.2

.1

.2

.3

.2

.7

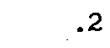

.7

.0

2.8
1955 Formula Best Este.

$0.0 *$

.2

1.1

1.7

1.4

$.3 *$

$.2 *$

$.1 *$

$.1 *$

$.2 *$

.4

.2

5.9
1956

Formula Best Est.

$0.1 *$

.5

$.4^{*}$

$.4^{*}$

1.1

.8

.4

.5

.6

.1

.1

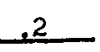

5.2
1957.

Eormula Best Est.

0.2

.2

.4

1.2

.8

.9

1.2

.8

1.8

1.2

.8

.2

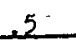

8.4

\section{8} Formula Best Est.

$0.7 * *$

Jan.

$.5 * *$

Feb.

March

Apri1

May

June

July

Aug.

Sept.

oct.

Nov.

Dec.

TOTAL
.5

1.4

1.8

1.1

1.0

.6

.5

1.7

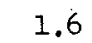

$-.8$

12.2

1959

Formula Best Est.

0.6

.8

1.9

2.2

1.1

1.7

.3

.2

.1

.1

\begin{tabular}{rc} 
Pre-1954 & 2.0 \\
1954 & 2.8 \\
1955 & 5.9 \\
1956 & 5.2 \\
1957 & 8.4 \\
1958 & 12.2 \\
1959 & 92 \\
\hline Total & 46
\end{tabular}

.1

.1

9.2 
1954

Eormula Best Rat.

Jen.

Feb.

Maroh

Apr11

May

June

July

Aug.

Sept.

oot.

Nov.

Deo.

TOTAI
0.00

.05

.02

.06

.03

.09

.05

.11

.29

.16

.18

.01

1.05
1955

Eormula Best Est.

$0.03 *$

. .14

1.23

3.64

1.46

3.04

1.23

$.14 *$

$.09 *$

$.08 *$

$.36 *$

$.10 *$

.13

.25

5.24
1956 Coxmula Best Est.

$0.11 *$

Eornula Best Est.

0.16

.39

.17

$.18 *$

.31

$.93 *$

1.05

1.07

.27

.19

.28

.34

.12

.09

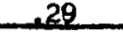

4.24
.43

.73

.17

.52

.81

47

25

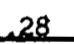

5.37

1958

2959

Forming Best Egt. Formuia Beat Bate.

Jon.

0.21

0.57

Feb.

.21

.88

Maroh

.25

.99

Apr11

2.63

May

.96

.74

June

.73

July

.35

1.27 .

.54

.12

.19

.09

Sept.

.30

oct.

.60

.08

Nov.

.46

.05

Pre-1954 1.02

$1954 \quad 1.05$

$1955 \quad 5.24$

$1956 \quad 4.24$

$1957 \quad 5.37$

$1958 \quad 5.46$

$1959 \quad 7.35$

Total

29.7

Deo.

TOTAL

.46

5.46

.06

.07

7.35 
STATION \#__..122

TOCATION ROCHESTER, NEW YORK

2954

1955

Formuli Best Est : Formula Best Esto

Jan.

0.0

Seb.

Marah.

April : :

May

June

Juty

Aug.

Sept.

oot.

Nov.

Deo.

TORAL

\section{.2}

.1

.3

.1

.3

.1

.3

.7

.4

.4

.0

2.8
$0.1 *$

.3

2.4

6.7

5.5

2.3

2.2

$.3 *$

$.2 *$

.$i *$

$.6 *$

$.2 *$

.2

.4

9.2
1956

Formula Best Est.

$0.2 *$

.6

$.3 *$

$1.4^{*}$

1.6

.5

.4

.6

.8

.2

.2

.6

7.4
1957

Formula Best Est.

0.3

.3

.5

2.0

.9

1.4

.8

.7

1.2

.5

.3

.5

9.4
1958 Formula Best Este.

0.4

Jan.

Feb.

March

April

May

June

July

Aug .

Sept:

oct.

Nov.

Dor.

OTAI.

1959

Formula Best Est.

1.2

1.8

1.9

4.7

2.2

.9

.2

.1

.1

.1

.1

.1

13.4 
STATION \# 127

LOCATION_NEW HAVEN, CONNECTICUT

1954 Eormula Best Est.

Jan.

Feb.

Maroh

Apr11

May

June

July

Aug .

Sept.

Oot.

Nov.

Deo.

TOTAL
0.04

.07

.03

.04

.02

.04

.06

.06

.23

.10

.39

.07

1.15
1952

Formule Best Est.

$0.05 *$

.45

$2.20 \quad 1.09$

$2.81 \quad 1.33$

$1.64 \quad .71$

$.20 *$

$.09 *$

$.11 *$

$.09 *$

$.23 *$

.05

.21

4.57
1956 Formula Best Est.

$$
0.23 *
$$

.45

$.54^{*}$

$.84^{*}$

.83

.23

.25

.14

.36

.12

.28

.31

4.48
1952

Formula Best Est.

0.19

.27

.58

2.29

.46

.32

.50

.76

.70

.47

.24

.52

7.30
0.53

.33

Feb.

March

Apr11

May

June

July

Aug.

Sept.

Oct.

Nov.

Dec.

TOTAL
.63

1.04

.78

.42

.24

.17

.23

.39

.66

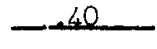

5.82
0.93

.75

1.89

2.45

.92

.72

.13

.10

.05

.09

.05

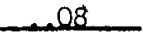

8.16

\begin{tabular}{rl} 
Pre-1954 & 1.21 \\
1954 & 1.15 \\
1955 & 4.57 \\
1956 & 4.48 \\
1957 & 7.30 \\
1958 & 5.82 \\
1959 & 8.16 \\
\hline
\end{tabular}


1954 Formula Best Est.

Jan. 0.0

Feb:

Marổh

Aprii

May

June

July

Aug.

Sept.

Oct.

Nov.

Deo.

TOTAL

.1

.1

.2

.1

.2

.2

.2

.6

.2

.9

3.0
1955 Formula Best Est. $0.1 *$

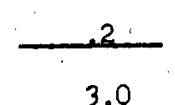

.9

$4.2 \quad 1.9$

5.2

3.0 .

2.4

1.3

$.4 *$

$.1 *$

$.2 *$

$.1 *$

$.4 *$

.1

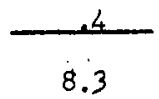

1956 Formula Best Est.

$0.4 *$

.7

$.8 *$

2. $2 *$

1.3

.5

.5

.3

.8

.2

.3

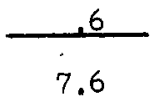

1957

Formula Best Est.

0.3

.4

.9

3.8

.8

.6

.8

1.2

$-1.1$

1.0

.5

.9

12.3

\section{$\quad 1958$}

Best Eat.

Cormula Best Est.

- Jan.

0.9

2.0

Feb.

.6

1.5

March

1.2

April

1.8

May

1.5

June

July

Aug.

Sept:

Oct.

Nov.

Dec.

TOTAL

.8

.6

.4

.5

1.5

1.9

$-1.0$

12.7
3.6

4.4

1.6

1.2

.2

.2

.1

.1

.1

.1

15.1 
STATION \# 132

IOCATION_JACKSONVILIE, FLORIDA

Eormela Best Est.

$$
0.04
$$

.06

Feb.

March

Apr11

May

June

JuIy

Aug.

Sept.

Oct.

Nov.

Deo.

TOTAL
.11

.07

.10

.12

.10

.08

.13

.09

.16

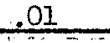

1.07
Eomula Best Est

$0.04 *$

.07

.57

.41

3.83

2.23

$.15 *$

$.09 *$

$.06 *$

$.05 *$

$.09 *$

.06

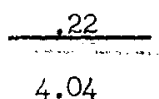

4.04
1956

Formula Best Est.

$0.11 *$

.24

$.27 *$

$.19 *$

.34

.19

.48

.24

.24

.32

.08

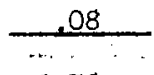

2.78
1957

Eormula Best Est.

0.10

.26

.38

.67

.31

.44

2.02

.79

1.48

.40

.13

.52

7.50

1958

Formula Best Est. Formula Best Est.

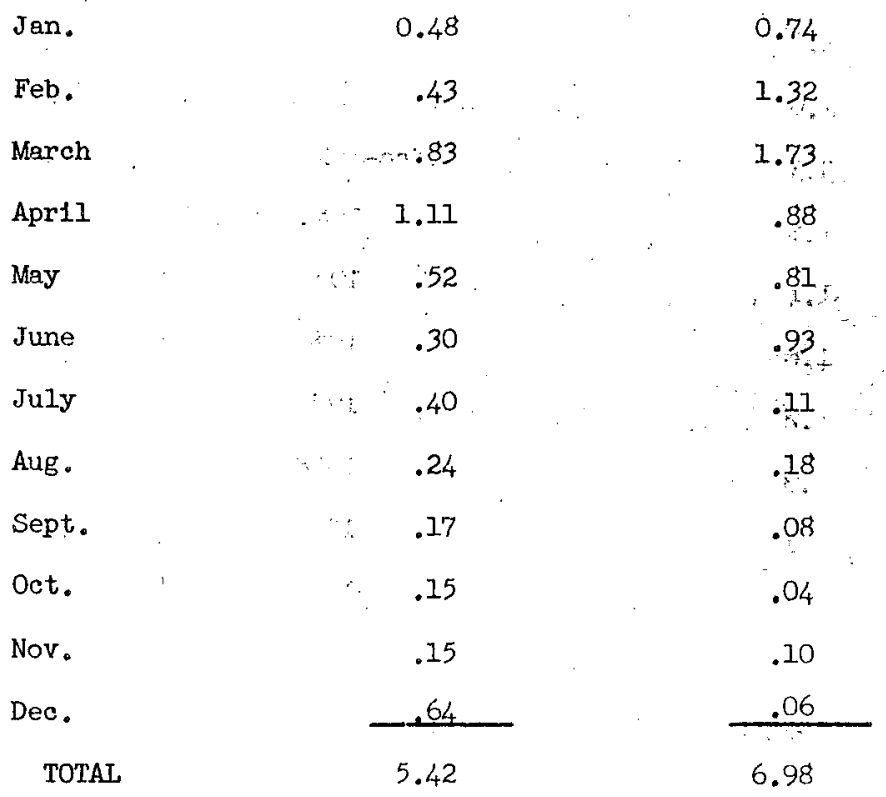

\begin{tabular}{rl} 
Pre-1954 & 0.92 \\
1954 & 1.07 \\
1955 & 4.04 \\
1956 & 2.78 \\
1957 & 7.50 \\
1958 & 5.42 \\
1959 & 6.98 \\
\hline Total & 28.7
\end{tabular}


STALION \# 132

1254 Formula Best Est.

Tan。

0.0

Feb.

Mares:

$A p r 37$

May

June

July

Aug.

Sept.

Det.

Nov.

Dec.

TOTAL

\section{.1}

.7

.4

.5

.5

.3

.2

.3

.2

.4

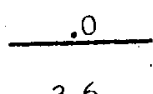

3.6

LOCATION

JACKSONVILLE, FLORIDA Formula Best Est. Eormula Best Est.

$0.1 *$

.1

1.1

.8

6.94 .0

$.3 *$

$.1 *$

$.1 *$

.1.*

$.1 *$

.1

.4

7.3
$0.2 *$

.4

$.4 *$

$.3 *$

.6

.4

1.1

.5

.5

.6

.2

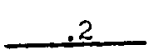

5.4
1957

Formula Best Est.

0.2

.4

.6

1.3

.5

.8

3.3

1.2

2.8

.9

.3

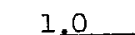

13.3

Formula Best Este Formula Best Est.

$\begin{array}{lcc}\text { Jan. } & 0.8 & 1.6 \\ \text { Feb. } & .7 & 2.6 \\ \text { March } & 1.7 & 3.3 \\ \text { April } & 1.9 & 1.6 \\ \text { May . } & 1.0 & 1.4 \\ \text { June } & .6 & 1.6 \\ \text { July } & 1.1 & .2 \\ \text { Aug. } & .6 & .3 \\ \text { Sept. } & .4 & .1 \\ \text { Oct. } & .5 & .1 \\ \text { Nov. } & .4 & .1 \\ \text { Dec. } & 11.2 & 13.1 \\ \text { ToTAL } & 11.2 & \end{array}$

Pre-1954 4.1

$1954 \quad 3.6$

$1955 \quad 7.3$

$1956 \quad 5.4$

$1957 \quad 13.3$

$1958 \quad 11.2$

$1959+13.1$

Total

58 


\section{ESTIMATE OF Sr $^{90}$ DEPOSITION FROM GUNADO FLU}

STATION \#- 133

LOCATION_MIAMI, FLORIDA

1954

1955

1956

1257 :

Formule Best Est. Formula Best Est. Eormula Best Este $\cdots$ Formula Best Est

Jan.

0.05

Feb :

March

April

May

June

Juily

Aug.

Seppt.

oöt.

Nóv.

Dêo.

TOTAL
.06 .

.15

.16

.36 .

.25

.21

.12

.10

.15

.19

.01

1.82
$0.02 *$

.41

.31

.61

4.04

2.10

$.02 *$

$.14 *$

.09*

$.04 *$

.09*

.08

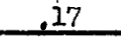

4.08
$0.17 *$

.05

$.12 *$

$.47 *$

.64

.19

.38

.34

.45

.30

.05

.08

3.24
0.13

. 21

.29

.25

.30

.30

1.25

1.17

.35

.45

.15

.22

5.07

1958

Formula Best Est. Formila Best Est.

Jan.

0.19

0.46

Feb.

.14

.73

March

.19

1.30

Apr11

.27

May

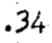

June

.22

July

Aủg.

Sept.

.41

.21

oct.

.22

Nov.

.13

Dec.

TOTAL

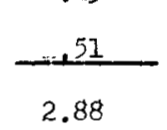

.68

.81

.42

.19

.12

.06

.07

.06

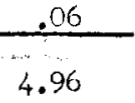

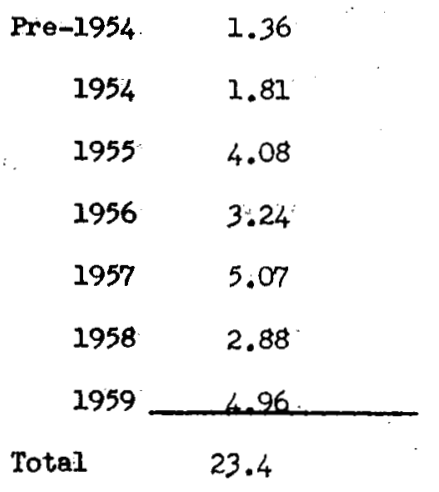




\begin{tabular}{|c|c|c|c|c|c|}
\hline . & Formuta Best Este & \multicolumn{2}{|c|}{ Eormula Best Este } & Eormula Best Est. & Formula Best Est. \\
\hline $\operatorname{Jan}$. & 0.1 & & $0.0 *$ & $0.3 *$ & 0.2 \\
\hline Feb. & .1 & & .8 & .1 & .3 \\
\hline March & .9 & & .6 & $.2 *$ & .5 \\
\hline April & .8 & & 1.1 & $.7 *$ & .5 \\
\hline May & 2.0 & 7.3 & 3.8 & 1.0 & .5 \\
\hline June & 1.0 & & $.0 *$ & .4 & .5 \\
\hline July & .7 & & $.2 *$ & .8 & 2.1 \\
\hline Aug. & .3 & & $.1 *$ & .7 & 1.9 \\
\hline Sept. & .3 & & $.1 *$ & 1.0 & .6 \\
\hline Oct. & .4 & & $.1 *$ & .6 & .9 \\
\hline Nov. & .4 & & .1 & .1 & .3 \\
\hline Dec. & مع. & & 3 & -2 & \\
\hline TOTAL & 7.0 & & 7.2 & 6.1 & \\
\hline
\end{tabular}

\begin{tabular}{|c|c|c|c|c|}
\hline & Formula Best Est. & Eormula Best Ekt. & & \\
\hline Jan. & 0.3 & 1.0 & & \\
\hline Feb. & .2 & 1.5 & & \\
\hline March & .4 & 2.5 & Pre-1954 & 5.9 \\
\hline April & .3 & 1.2 & 1954 & 7.0 \\
\hline May & .7 & 1.4 & 1955 & 7.2 \\
\hline June & .4 & .7 & 2956 & 6.1 \\
\hline July & .4 & .3 & 1957 & 8.7 \\
\hline Aug. & 1.0 & .2 & 1958 & 6.5 \\
\hline Sept. & .5 & .1 & 1959 & 9.2 \\
\hline Oct. & .7 & .1 & Totel & 51 \\
\hline Nov. & .4 & .1 & & \\
\hline Dec. & 1.2 & $\therefore .1$ & & \\
\hline TOTAL & 6.5 & 9.2 & & \\
\hline
\end{tabular}


ESTIMATE OF Sr 90 :DEPOSITION FROM GUMMED FILM

STATION \#- 134

LOCATION WASHINGTON, D.C. SILVER HIIJ

\section{$\therefore \quad 1954$}

Eormula Best Est.

Jan.

Feb.

March

April

May

June

JuIy

Aug.

Sept.

Oct.

Nov.

Dec.

TOTAL
0.00

.07

.04

.04

.04

.09

.06

.10

.09

$.08 * *$

$.36 * *$

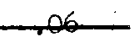

1.03
. 1955

Fermula Best Este.

$0.07 *$

.19

$.93 \quad 0.50$

1.530 .62

$2.85 \quad 1.67$

.29

$.09 *$

$.11 *$

$.43 *$

$.10 *$

.12

27

4.42
2956

Eormula Best Eat. Eormule Best Est.

$0.20 *$

.39

$.56 *$

$.62 *$

1.17

.24

.24

.14

.40

.25

.17

.13

4.51
0.26

.32

.47

.56

.35

.64

.47

.28

.59

.27

.14

.32

4.67

\section{8}

1959

Formula Best Este Formule Best Est.

Jan.

Feb.

March

April

May

June

July

Aug .

Sept.

oct.

) Nov.

Dec.

TOTAL
0.34

.33

.48

1.16

.33

.35

.25

.21

.15

.26

.44

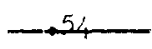

4.84
0.70

1.03

1.07

$2.34 \ldots$

.72

$.48:$

.11

$.08 \quad \therefore$

.05

.05

.04

08

6.75 


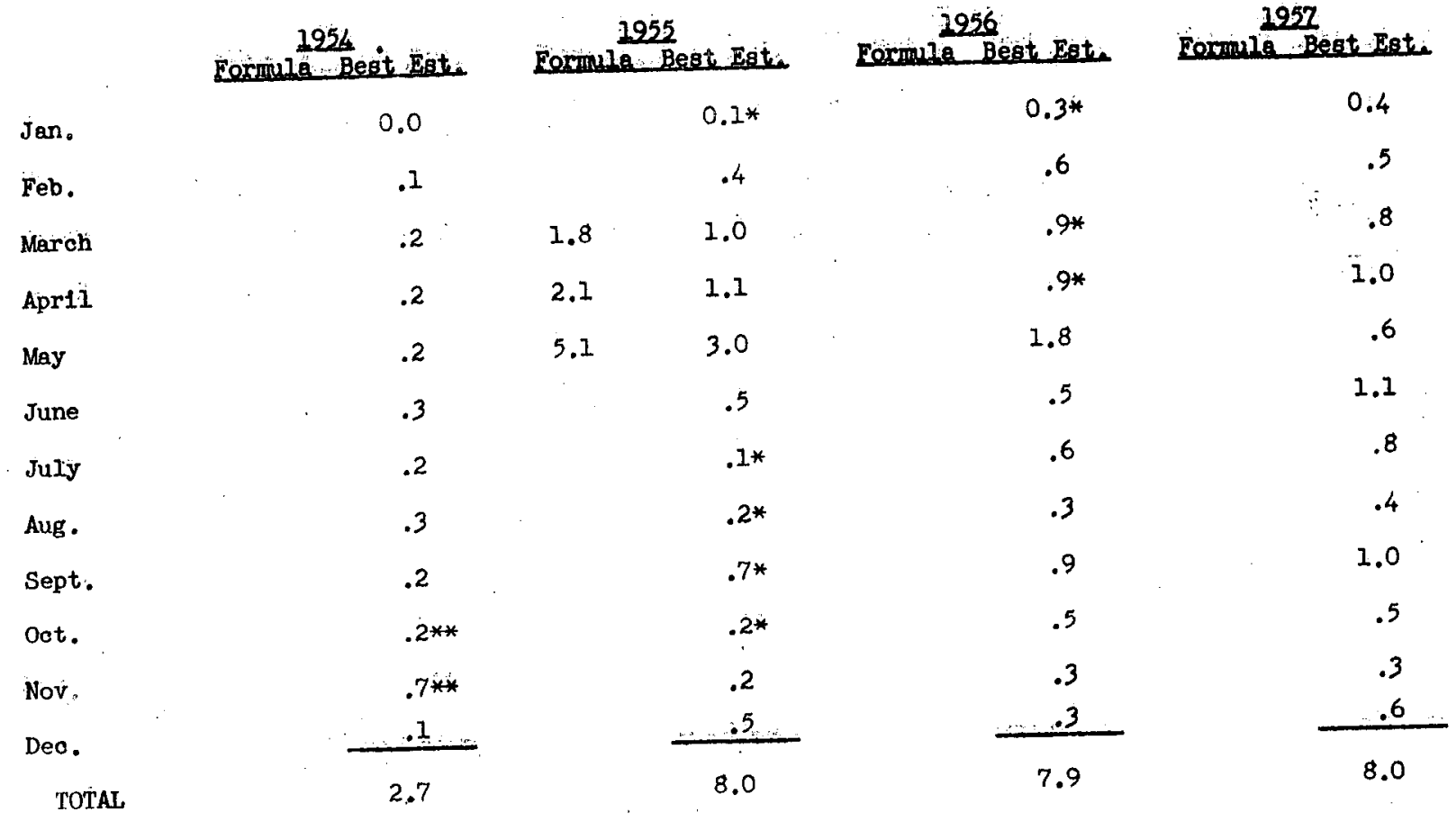

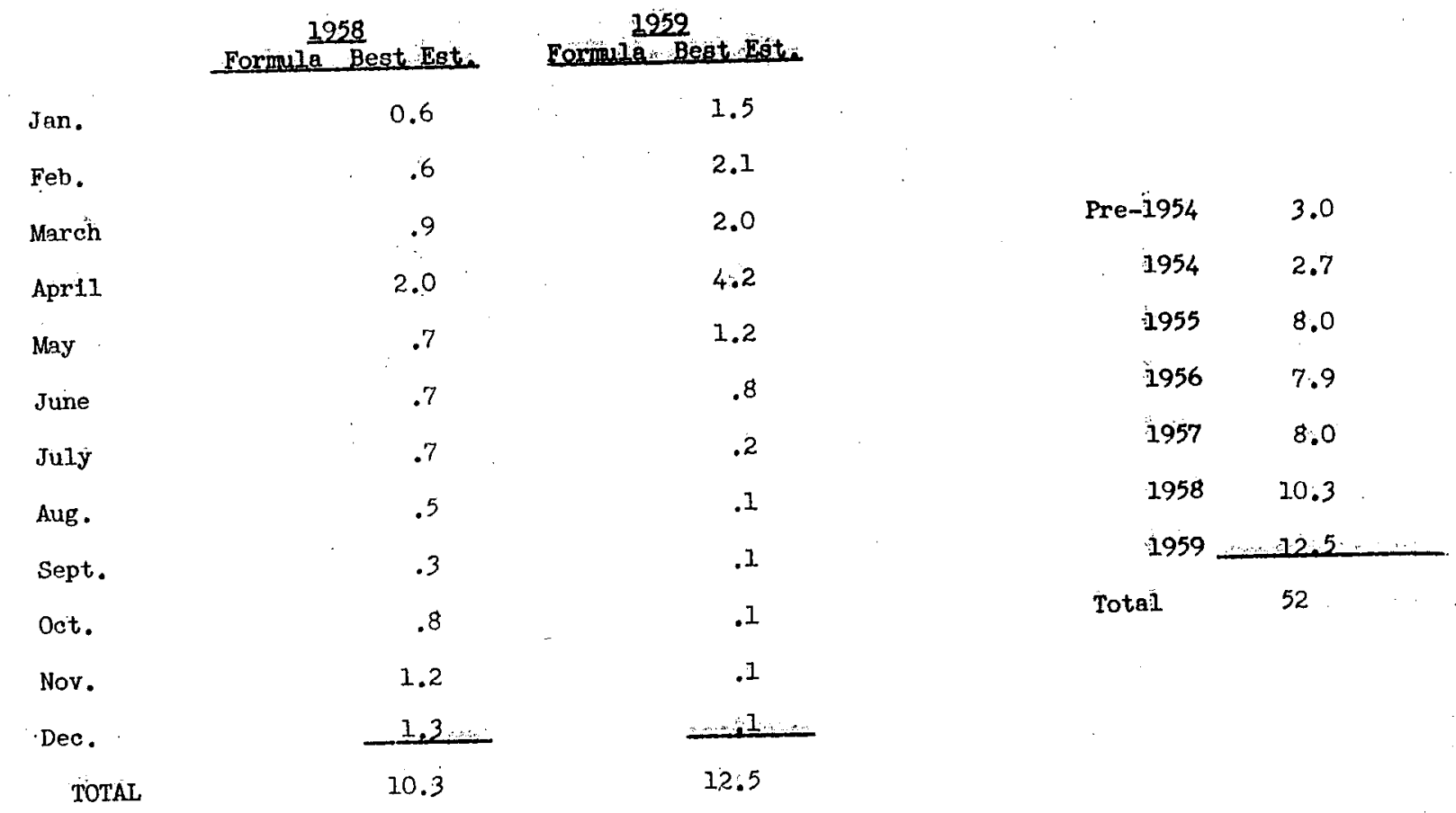




\section{ESTIMATE OF Sr 90 DEPOSITION FROM GUMMED FILM}

STATION \# 137

LOCATION CLEVETAND, OHIO

Formula Best Est.

Jan.

Feb.

March

April

May

June

July

Aug.

Sept.

oct.

Nov.

Dec.

TOTAL
0.09

.09

.04

.14

.07

.15

.07

.12

.18

.34

.18

.31

1.74
1925

Formula Best Est.

$0.08 *$

.34

3.46

10.44

1.92

3.20

0.95

$.31 *$

$.20 *$

$.01 *$

$.08 *$

$.06 *$

.06

.25

6.38
1956

Formule Best Est.

$0.25 *$

.39

$.32 *$

$.40 *$

1.07

.81

.36

.42

.70

.14

.13

.21

5.20
1957

Formula Best Est.

.29

.30

.81

2.28

.44

.65

.71

.33

.76

.52

.12

.32

7.53

1958

1259.

Formula Best Est. Formula Begt Egt.

Jan.

0.73

0.66

Feb.

.29

.96

March

.24

1.39

April

1.72

May

.74

June

.65

July

.35

Aug.

.30

3.05

1.89

.42

Sept.

.27

Oct.

.61

Nov.

Dec.

.71

.18

.17

$\therefore .08$

.05

.09

TOTAL

.40

7.01

.03

8.97

\begin{tabular}{rl} 
Pre-1954 & 1.37 \\
1954 & 1.74 \\
1955 & 6.38 \\
1956 & 5.20 \\
1957 & 7.53 \\
1958 & 7.01 \\
1959 & 8.97 \\
\hline Total & 38.2
\end{tabular}


STATION \# 137

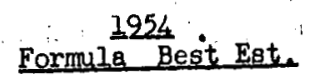

Jan。

0.1

Feb.

March

April

May

June

July

Aug.

Sept.

Oct.

Nov.

Deo.

TOTAL
.2

.2

.7

.4

.6

.2

.3

.5

.8

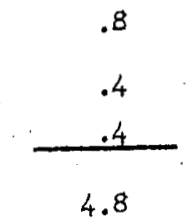

$\quad 2955$

Formula Best Est.

$0.2 *$

.7

6.7

1.6

19.4

7.5

3.5

1.7

$.5 *$

$.3 *$

. $\mathrm{O} *$

$.1 *$

. $1 *$

.1

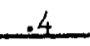

13.2
1956 Eermula Best Est. $0.4 *$ .6 $.5 *$ $.6 *$

1.9

1.4

.6

.7

1.2

.2

.2

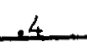

8.7
1957

Formula Best Est.

.5

.5

1.4

4.1

.7

1.2

1.2

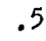

1.2

1.1

.2

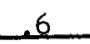

13.2

1959

Formula Best Este Formula Best Est.

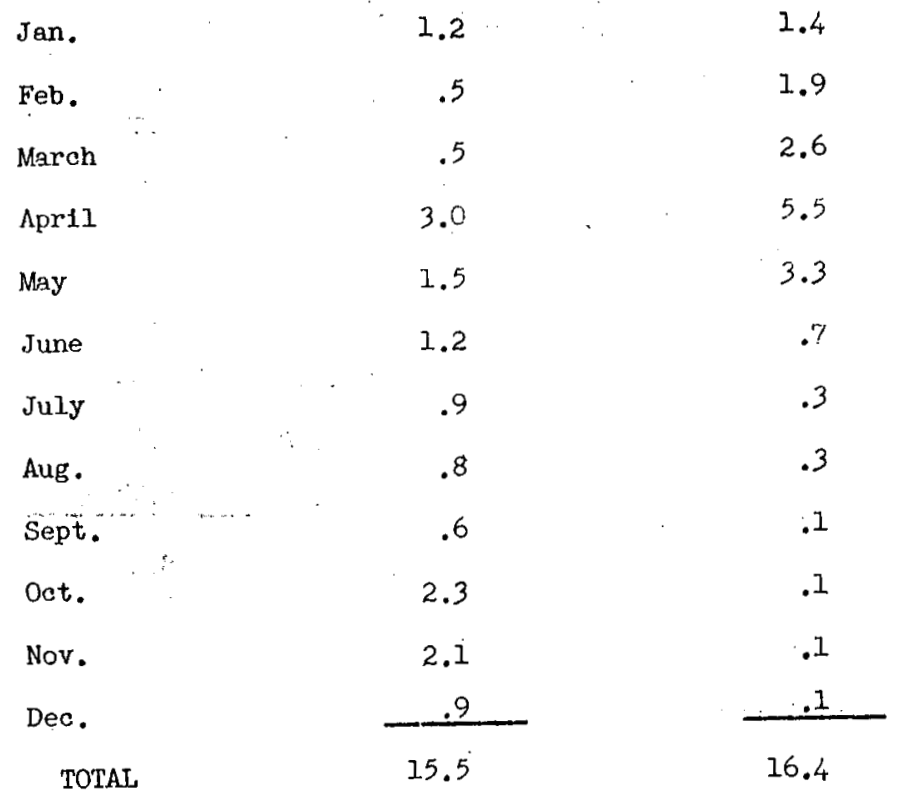

\begin{tabular}{rr} 
Pre-1954 & 5.2 \\
1954 & 4.8 \\
1955 & 13.2 \\
1956 & 8.7 \\
1957 & 13.2 \\
1958 & 15.5 \\
1959 & 16.4 \\
\hline Total & 77
\end{tabular}$$
5.5
$$ 
STATION \# 138

\section{4}

Formula Best Est.

Jan.

Feb.

March

April

May

June

July

Aug.

Sept.

Oct.

Nov.

Deo.

TOTAL
0.05

.04

.06

.02

.04

.04

.09

.27

.17

.07

.33

$.03 * *$

1.21
Formula Best Est.

$0.02 *$

.11

.65

.37

2.16

$.14 *$

$.06 *$

$.05 *$

$.04 *$

$.103 *$

.21

.22

4.06 .

LOCATION CAPE HATTERAS, NORTH CAROLINA

1956 Formula Best Est.

$0.65 *$

.53

$.29 *$

$.24 *$

.15

.26

.27

.27

.28

.25

$\begin{array}{r}.09 \\ .06 \\ \hline 3.24\end{array}$

1957

Formula Best Est.

.42

.50

.56

.32

.25

.28

$2.12 \quad 1.20$

.60

1.14

.35

.28

.51

6.41

\section{8}

Formula Best Este Formula Best Est.

Jen.

0.24

0.60

Feb.

.25

1.54

March

.39

April

.60

May

.17

.22

Junẹ

.10

July

.15

Aug.

.24

1.71

1.60

.77

.22

.23

.04

Sept.

.13

.09

Oct.

.32

.06

\begin{tabular}{rl} 
Pre-1954 & $1.23 * *$ \\
1954 & 1.21 \\
1955 & 4.06 \\
1956 & 3.24 \\
1957 & 6.41 \\
1958 & 3.25 \\
1959 & 6.95 \\
\hline Totel & 26.4
\end{tabular}

Nov.

Dec.

TOTAL

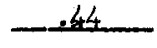

.04

3.25

.05

6.95 
STATION \# 138

LOCATION_CAEE HATLERAS, NORTH CAROLTNA

1954 Formula Besit Est.

Jan.

Feb.

March

Apri1

May

June

July

Aug.

Sept.

oct.

Nov.

Deo.

TOTAL
.I

.1

.3

.1

.2

.1

.3

.8

.4

.2

.7

$.1 * *$

3.4
1955 Eormula Best Est.

\begin{tabular}{c}
$0.0^{*}$ \\
.2 \\
1.3 \\
.7 \\
3.9 \\
$.2 *$ \\
$.1 *$ \\
$.1 *$ \\
$.1 *$ \\
$.0 *$ \\
.4 \\
.4 \\
\hline .4
\end{tabular}

1956 Eormula Best Est.

$1.0 *$

.9

$.4^{*}$

$.4^{*}$

.3

.5

.5

.5

.3

.4

.2

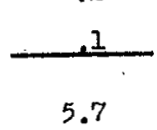

1957

Formula Best Ist.

.7

.9

1.0

.6

.4

.5

2.0

.9

2.2

.7

.6

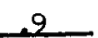

11.4

1958

1959

Formula Best Este. Formule Best Est.

Jan.

Feb.

March

Apr11

May

June

July

Aug .

Sept.

Oct.

Nov.

Dec .

TOTAL
0.4

.4

.8

1.0

.3

.4

.2

.4

.6

.4

.9

1.0

6.8
1.3

3.1

3.2

2.9

1.3

.4

.4

.1

.1

.1

.1 .

13.1

\begin{tabular}{rr} 
Pre-1954 & $4.8 * *$ \\
1954 & 3.4 \\
1955 & 7.4 \\
1956 & 5.5 \\
1957 & 11.4 \\
1958 & 6.8 \\
1959 & 13.1 \\
\hline
\end{tabular}

Total

52 


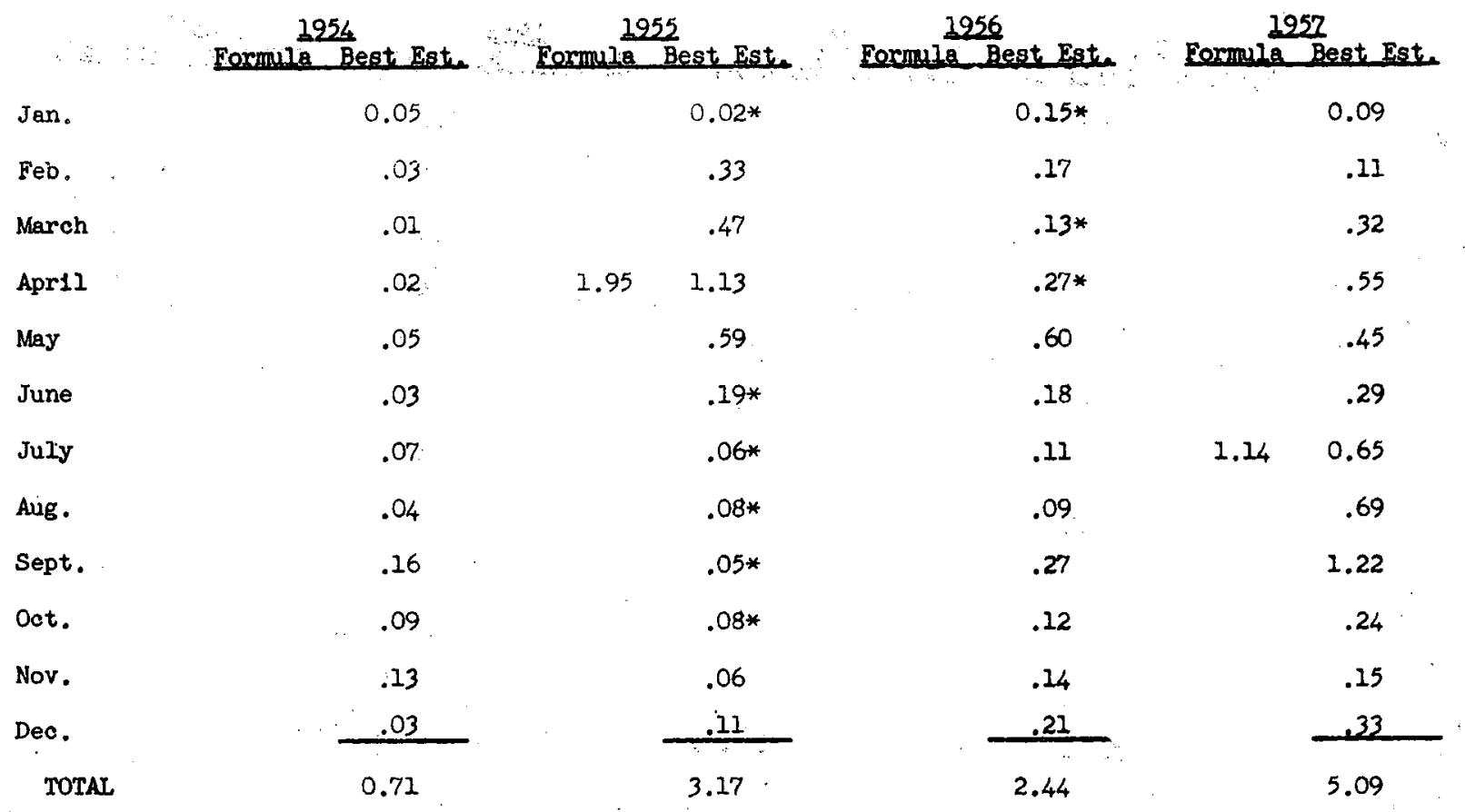




\section{ESTTMATE OF INFINITY $y$ DOSE GROM GOMMOD TILU}

STATION "I39

LOCATION CONCORD. NAII HAMPSHTRE

1956 Eormin Best Eato

Jan。

0.1

Eeit.

.1

March

.1

Apr11

May

.1

.2

June

.1

JuIy

.2

Aug.

Sept.

oct.

Noy.

Deo.

TOTAL

.1
.1
.1
.2
.1
.2
.1
.4
.2
.3
.0
1.9

1955

$0.0 *$
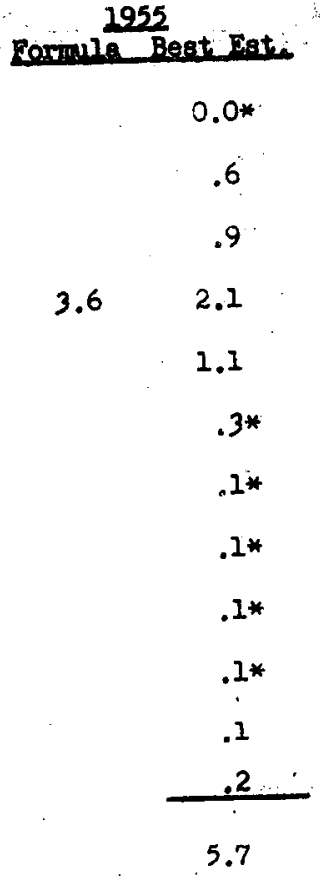

Tormula Best Eati.

$0.2 *$

.3

.2*

$.4 *$

.9

.4

.3

.2

.6

.2

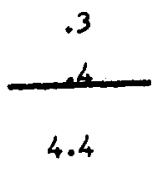

2952

Bomula Best Eat.

0.2

.2

.5

.9

8

.5

$1.9 \quad 1.1$

1.1

1.9

.6

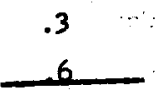

8.7
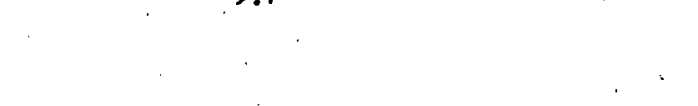

1959

0.3

Jan.

Feb.

March

Apr11

May

June

July

Aug.

sept.

oct.

Nov.

Dec.

TOTAL
.2

.8

1.2

1.0

.6

.5

.3

.4

.9

1.4

$\frac{.8}{8.4}$
1.0

.8

1.7

3.0

1.1

1.4

.2

.1

.1

.1

.1

1

9.7 


\section{ESTIMATE OF Sr 90 DEPOSITION FROM GUMMED FIIM}

STATION \# 141

LOCATION_BOSTON, MASSACHUSETTS

\section{4}

Formula Best Est.

1955

Eormula Best Est.

Jan.

0.19

$0.03 *$

1956

Formula Best Est.

Formile Begt Est.

Feb.

.19

March

.03

.42

$0.33 *$

0.22

April

May

.03

.100

2.22

1.13

.02

June

.07

JuIy

.09

Aug.

.09

Sept.

.40

Oct.

.46

Nov.

.39

Deo.

.01

TOTAL

1.97

.98

.43

$.16 *$

$.12 *$

$.06 *$

$.18 *$

.07

.15

.36

.24

$.34 *$

.53

$.27 *$

.92

.63

.62

.30

.50

.17

.50

.11

.42

.47

.60

.30

.22

3.83 .

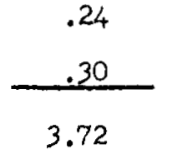

$\frac{.32}{5.27}$

Formula Best Est.

0.60

1959

Jen,

.30

Formula Best Est.

Feb.

.75

March

1.11

April

1.87

.2 .75

May

.79

.81

June

.54

July

.29

Aug.

.25

Sept.

.28

oct.

.32

Nov.

.53

Dec .

.55

TOTAL

7.07

.68

.15

.12

.05

.05

.04

.10

7.33 


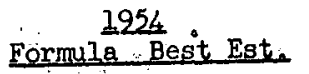

Jan.

Feb.

March

April

May

June

July

Aug.

Sept.

Oot.

Nov.

Dec.

TOTAL
0.2

.3

.2

.2

.1

.3

.3

.2

1.0

1.1

.9

.0
1955

Eormula Best Est.

$0.1 *$

.8

1.9

4.1

2.2

1.8

.7

.3*

$.2 *$

$.1 *$

$.3 *$

.1

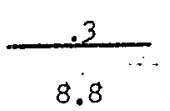

1956

Formula Best Est.

$0.5 *$

.6

$.5 *$

$.4 *$

1.0

.6

.4

.2

1.0

.4

.5

.6

6.7
1952

Formula Best Est.

0.4

.4

.8

1.6

1.1

.7

.8

.7

1.0

.7

.4

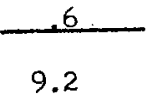

1958

1959

Formula Best Est. Formula Best Est.

Jan.

Feb.

March

Apri1

May

June

July

Aug.

Sept.

Oct.

Nov.

Dec.

TOTAL
1.0

.6

1.5

3.2

1.6

1.0

.8

.6

.7

1.2

1.6

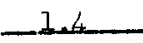

15.2
1.6

1.5

2.1

5.0

1.4

1.1

.2

.2

.1

.1

.1

1

9.0 
STATION \#20\%

LOCATION CORPUS CHRISTI, TEXAS

. 1924

Formula Best Est.

Jan.

Feb.

March

Apr11

May

June

JuIy

Aug .

Sept.

oot.

Nov.

Deo.

TOTAL
0.04

.12

.16

.17

.19

.08

.05

.05

.09

.10

.11

.00

1.16
Formula Best Est.

$0.07 *$

.34

.26

.65

$1.90 \quad .99$

$.07 *$

$.06 *$

$.04 *$

$.08 *$

$.06 *$

.18

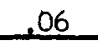

2.86
1956 Formula Best Est.

$0.12 *$

.08

$.11 *$

$.14 *$

.23

.08

.14

.23

.27

.26

.19

.13

1.87

1957

Formula Best Est.

0.20

.23

.42

.32

.38

.70

.22

.33

.37

.37

.16

.30

4.00

1959

Formula Best Eat. Eormula Best PBt.

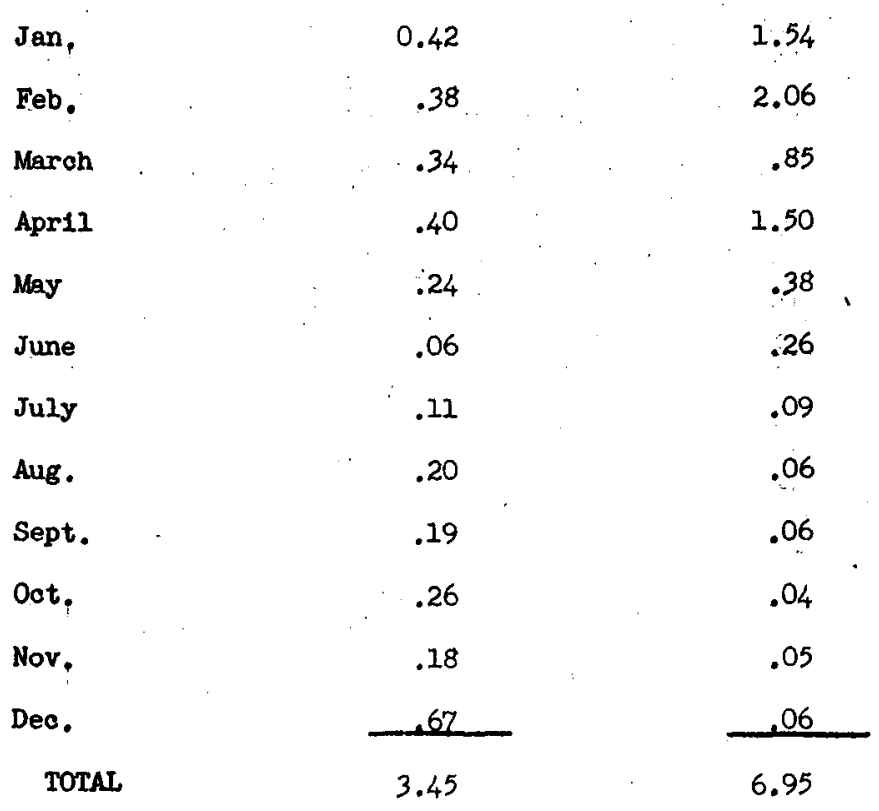

\begin{tabular}{rl} 
Pre-1954 & 0.87 \\
1954 & 1.16 \\
1955 & 2.86 \\
1956 & 1.87 \\
1957 & 4.00 \\
1958 & 3.45 \\
1959 & 6.95 \\
\hline & 21.2
\end{tabular}


STATION \#_ 204

LOCATION_ CORPUS CHRISTI, TEXAS

1254 . Formula Besit Est.

Jan.

Feb.

March

April

May

June

JuIy

Aug .

Sept.

Oct.

Nov.

Dec.

TOTAL
0.0

.2

.8

1.0

1.1

.3

.2

.1

.2

.2

.3

.0

4.4
1955

Formula Best Est.

$0.2 *$

.7

.5

1.2

3.4

1.7

.1*

. I*

. . *

$.1 * *$

$.1 *$

.3

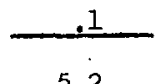

5.2
1956

Formula Best Est.

$0.2 *$

.1

$.2 *$

$.2 *$

.3

.2

.3

.5

.6

.3

.4

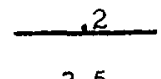

3.5
2957

Formula Best Est.

0.3

.4

.7

.6

.6

1.4

.4

.5

.6

.9

.4

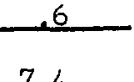

7.4
1958 Formula Best Est.

Jan.

Feb.

March

Apri1

May

June

July

Aug.

Sept.

oct.

Nov.

Dec.

TOTAI

$\begin{array}{r}0.7 \\ .6 \\ .7 \\ .7 \\ .5 \\ .1 \\ .3 \\ .5 \\ .4 \\ .9 \\ .5 \\ 1.6 \\ \hline 7.5\end{array}$

0.7

Formula Best Est.

3.3

4.1

1.6

2.7

.7

.4

.1

.1

.1

.1

.1

.1

13.4

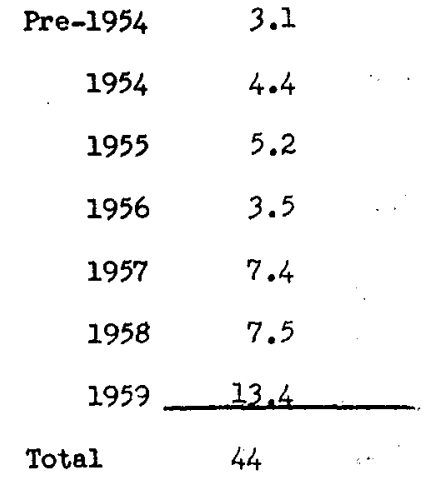


1954 Eormula Best Est.

Jan.

Feb。

March

April

May

June

July

Aug .

Sept.

Oot.

Nov.

Deo.

TOTAL
0.04

.14

.17

.18

.52

0.34

.17

.10

.05

.05

.27

.10

.02

1.63
1955

Formula Best Est.

$0.03 *$

.17

1.75

.90

.87

25.56

6.21

$.20 *$

$.08 *$

$.08 *$

$.07 *$

$.12 *$

.03

.10

9.06
1956 Formula Best Est.

$0.22 * *$

.40

$.29 *$

$.64 *$

.42

.23

.94

.20

.11

.22

.12

.14

3.93
1957

Fomula Best. Est.

0.15

.34

.85

2.53

.61

.96

.37

.49

.80

.47

.27

$.38 \quad .23$

8.07

1958 1259

Formula Best Est. Formile Best Eat.

$\begin{array}{lcc}\text { Jan. } & 0.48 & 0.48 \\ \text { Feb. } & .38 & .95 \\ \text { March } & .81 & 1.67 \\ \text { Apr1l } & .93 & 1.58 \\ \text { May } & .33 & 1.58 \\ \text { June } & .46 & .46 \\ \text { July } & .26 & .12 \\ \text { Aug. } & .32 & .10 \\ \text { Sept. } & .11 & .06 \\ \text { Oct, } & .19 & .05 \\ \text { Nov, } & .27 & .06 \\ \text { Dec. } & .31 & .05 \\ \text { ToTAL } & 4.85 & \end{array}$

\begin{tabular}{rl} 
Pre-1954 & 1.84 \\
1954 & 1.63 \\
1955 & 9.06 \\
1956 & 3.93 \\
1957 & 8.07 \\
1958 & 4.85 \\
1959 & 7.16 \\
\hline Total & 36.5
\end{tabular}

$\int \operatorname{TOTAL}$ 


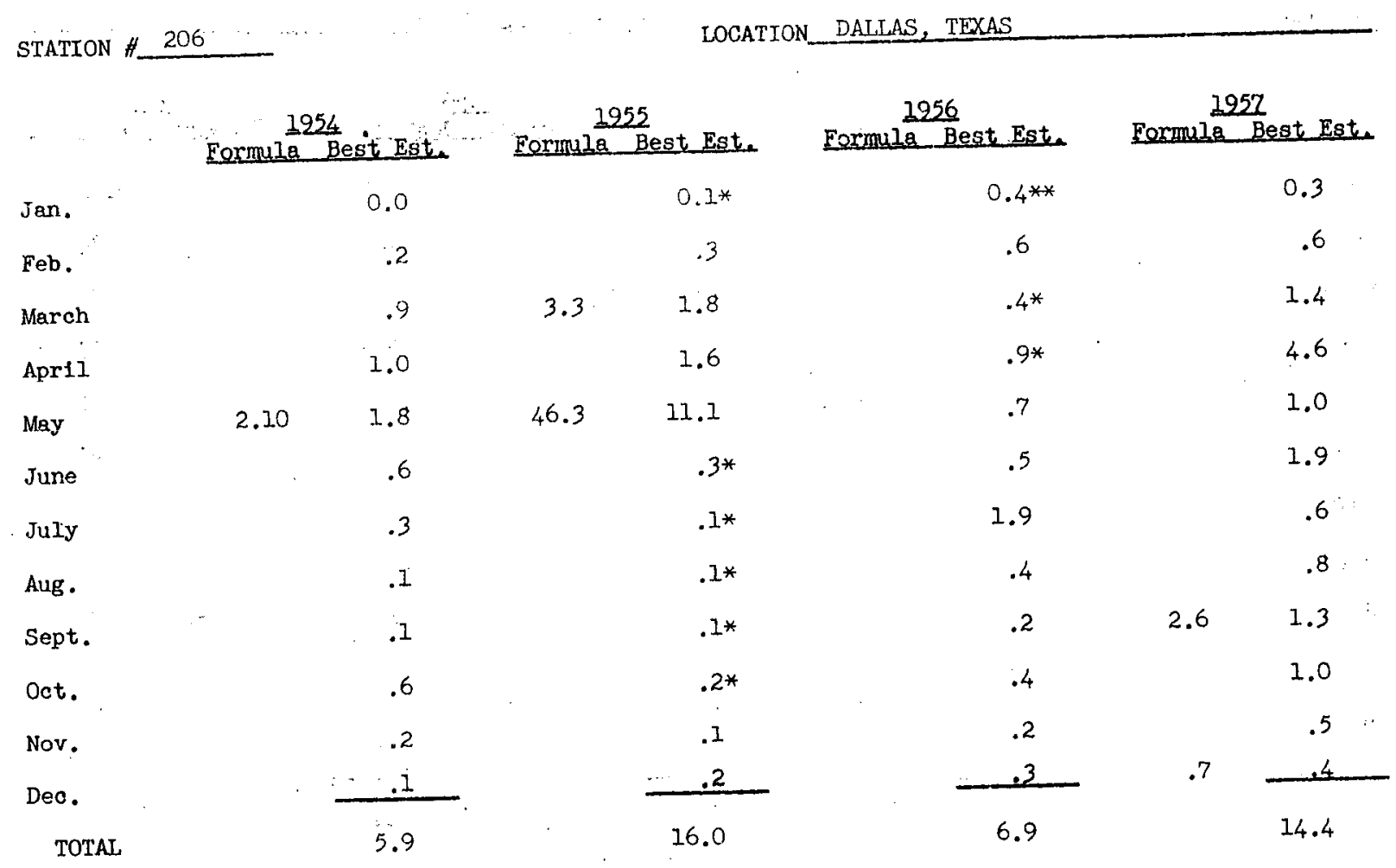

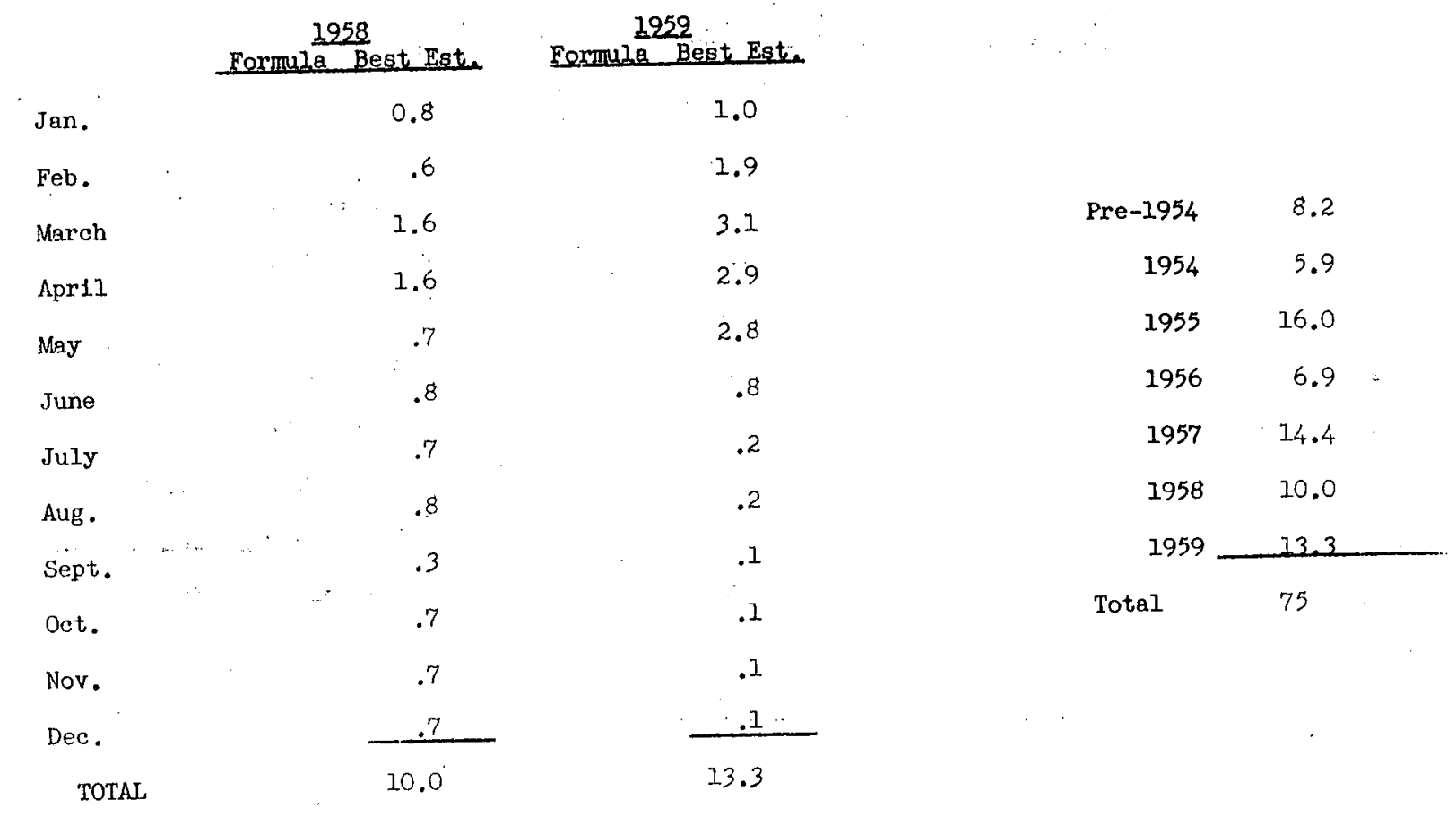


STATION \#-209

1954

Formula Best Est.

Jan.

Feb.

March

Aprt1

May

June

July

Aug .

Sept.

oct.

Nov.

Deo.

TOTAL
0.04

.29

.07

.07

.40

.21

.09

.14

.10

.14

.06

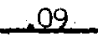

1.70
1955

Formula Best Est.

$0.03 *$

.27

1.67

0.61

6.20

2.23

4.17

1.65

$.38 *$

$.09 *$

$.03 *$

$.09 *$

$.06 *$

.05

12

5.57
1956

Formula Best Est

$0.17 *$

.24

$.28 *$

$.27 *$

.72

.22

.94

.30

.40

.13

.09

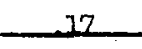

3.93
1957

Eormula Best Est.

0.17

.21

.61

1.05

1.56

$1.49 \quad 1.04$

.76

.48

$4.31 \quad 1.65$

.46

.26

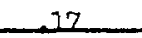

8.42

\section{$2958 \quad 2952$}

Formula Best Esta Formule Best Est.

Jan,

Feb.

March

Apr11

May

June.

JuIy

Aug.

Sept.

Oct;

Nov,

Dec.

TOTAL
0.13

.28

.36

1.73

.49

.67

.71

.28

.34

.22

.18

.31

5.70
0.28

.46

1.11

1.75

1.08

.48

. .23

.08

.06

.07

.05

.02

5.67

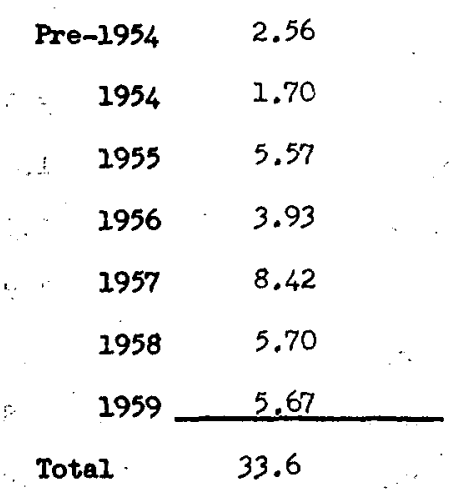

Pre-1954 2.56

$\therefore \quad 1954 \quad 1.70$ 
STATION \#_ 209

IOCATION_WICHITA, KANSAS

$1954 \quad 1955$ Formuli Best Est.

Formule Best Est.

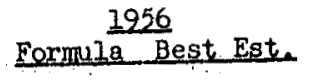

$0.3^{*}$

Feb.

March

Apr11

May

June

JuIy

Aug.

Sept.

Oct.

Nov.

Dec.

TOTAL
0.0

.5

.4

.4

2.4

.8

.3

.4

.2

.3

.1

.3

6.1
.5

$0.1 *$

3.21 .1

11.4

7.6

4.1

3.0

$.7 *$

$.1 *$

. $.1 *$

$.1 *$

$.1 *$

.1

.2

20.2
.4

$.4 *$

$.4^{*}$

1.3

.4

2.3

.7

.9

.2

.2

3

7.8
1957

Eormula Best Est.

0.3

.4

1.0

1.8 .

2.6

$2.7 \quad 1.8$

1.3

.7

$6.7, \quad 2.7$

1.1
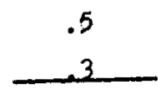

14.5

Formula Best Est.

Jan.

Feb.

March

Apr11

May

June

July

Aug.

Sept.

Oct.

Nov.

Dec.

TOTAL
0.2

.5

.7

3.0

1.0

1.3

1.9

.7

.8

.8

.5

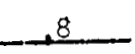

12.2
.6

.9

2.1

3.2

1.9

.8

.4

.1

.1

.1

.1

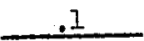

10.3

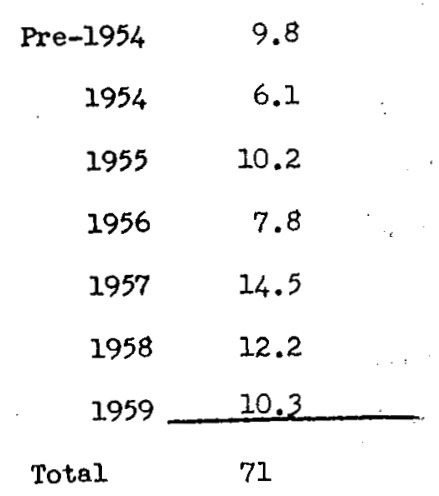

Pre-1954 9.8

6.1

$1956 \quad 7.8$

$1957 \quad 14.5$

$1958 \quad 12.2$

71 


\section{ESTIMATE OF SY ${ }^{90}$ DEPOSITION FROM CLMMED FILM}

STATION \#211

IOCATION_SCOTTSBLUUEF, NEBRASKA

2954

Eormula Best Est.

Jan.

Feb.

Merch

Apr11

May

June

JuIy

Aug.

Sept.

Oot.

Nov.

Dec.

TOTAL
0.04

.10

.02

.10

.31

.27

.21

.24

.17

.20

.08

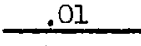

1.75
1952

Eormula Best Est

$0.04 *$

.17

$2.48 \quad 1.36$

$1.86 \quad 1.02$

$8.13 \quad 1.43$

$.29 *$

$.23 *$

$.14 *$

$.08 *$

$.11 *$

.27

.24

5.38
1956 Eormula Best Est.

$0.14 *$

.19

$.20 *$

$.51 *$

.15

.58

1.35

.47

.44

.16

.09

.17

4.45
1957

Eormula Best Est.

$$
0.14
$$

.20

.26

.78

2.05

$1.60 \quad .86$

$3.07 \quad 1.60$

.86

1.53

.70

.13

36

8.37

\begin{tabular}{lcc} 
Jan, & 0.16 & 0.36 \\
Feb. & .17 & .45 \\
Maroh & .37 & 1.08 \\
April & .68 & 1.23 \\
May & .88 & 2.64 \\
June & 1.13 & 1.21 \\
July & .73 & .31 \\
Aug. & .27 & .11 \\
Sept. & .30 & .09 \\
Oct, & .24 & .05 \\
Nov, & .36 & .06 \\
Dec. & .57 & .08 \\
\hline Total & 5.86 & 7.67
\end{tabular}

\begin{tabular}{rr} 
Pre-1954 & 1.71 \\
1954 & 1.75 \\
1955 & 5.38 \\
1956 & 4.45 \\
1957 & 8.37 \\
1958 & 5.86 \\
1959 & 7.67 \\
\hline Total & 35.2
\end{tabular}


STATION \# 211

LOCATION SCOTTSBLUFF, NEBRASKA

1954 Formula Best Este.

Jan.

Feb.

March

April

May

June

July

Aug.

Sept.

Oct.

Nov.

Dec.

TOTAL
0.0

.2

.1

.5

1.8

1.0 .

.7

.7

.4

.5

.2

.1

6.2
Formula 1955 cormula Begt Est.

$0.1 *$

.3

4.8

2.6

3.5

1.8

14.8

2.6

$.5 *$

$.4 *$

$.2 *$

$.1 *$

$.2 *$

.5

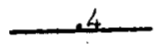

9.7
1956 Eormula Best Est.

$0.2 *$

.3

$.3 *$

$.8 *$

.3

1.1

3.0

1.0

.9

.3

.2

3

8.7
1957

Formula Best Est.

0.3

.2

.4

1.5

1.8

1.6

2.7

1.4

2.4

2.4

.3

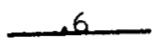

14.6
Formula Best Est.

Jan.

0.3

Feb.

March

April

May

June

July

Aug .

Sept.

Oct.

Nov.

Dec.

TOTAL
.3

.7

1.2

1.7

2.2

2.0

.7

.7

.9

1.1

1.4

13.2

1952

Formula Best Est.

0.8

.9

2.0

2.2

4.6

2.0

.5

.2

.1

.1

Pre-1954 $\quad 4.8$

$1954-6.2$

$1955 \quad 9.7$

$1956 \quad 8.7$

$1957 \quad 14.6$

$1958 \quad 13.2$

$1959+13.6$

Total

71

.1

.1

13.6 
1954 Formula Best Est. 1955

Jan.

Feb.

March

April

May

June

July

Aug.

Sept.

Oct.

Nov.

Dec.

TOTAL

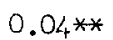

.06

.02

.02

.16

.19

.18

.20

.13

.10

.07

$.03 *$

1.20
Formia Best Est.

$0.02 *$

.20

.34

.57

$2.79 \quad 1.14$

$.47 *$

$.27^{*}$

.1.9*

$.35 *$

$.3 .3 *$

.05

.17

3.70
1956

Formula Best Est

$0.08 *$

$.21 * *$

$.15 *$

$.24 *$

.52

.37

.89

.43

.44

.20

.02

.13

3.68
1957

Formula Best Est.

0.08

.09

19

.44

60

.57

$10.70 \quad 3.70$

.82

.87

.44

.06

.39

8.25

\section{8}

1959

Formula Best Est. Eormila Best Est.

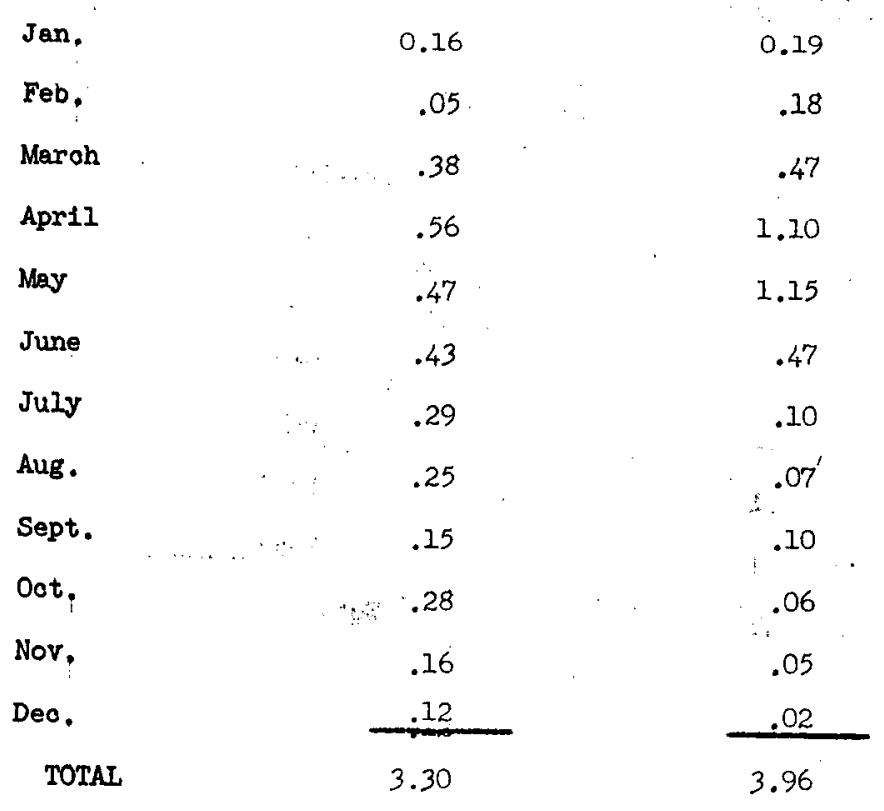

\begin{tabular}{rl} 
Pre-1954 & 2.05 \\
1954 & 1.20 \\
1955 & 3.70 \\
1956 & 3.68 \\
1957 & 8.25 \\
1958 & 3.30 \\
1959 & 3.96 \\
\hline Total & 26.1
\end{tabular}




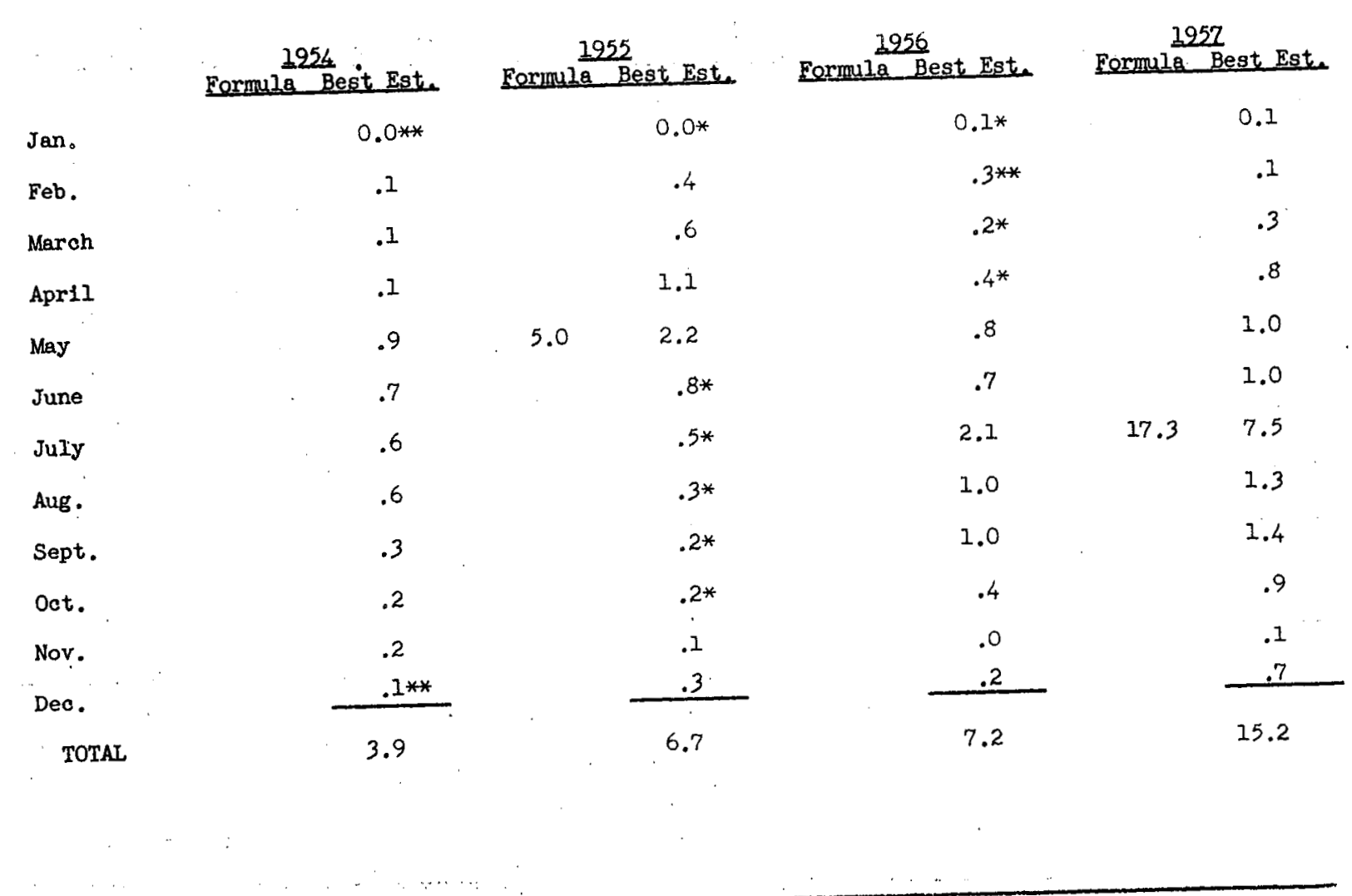

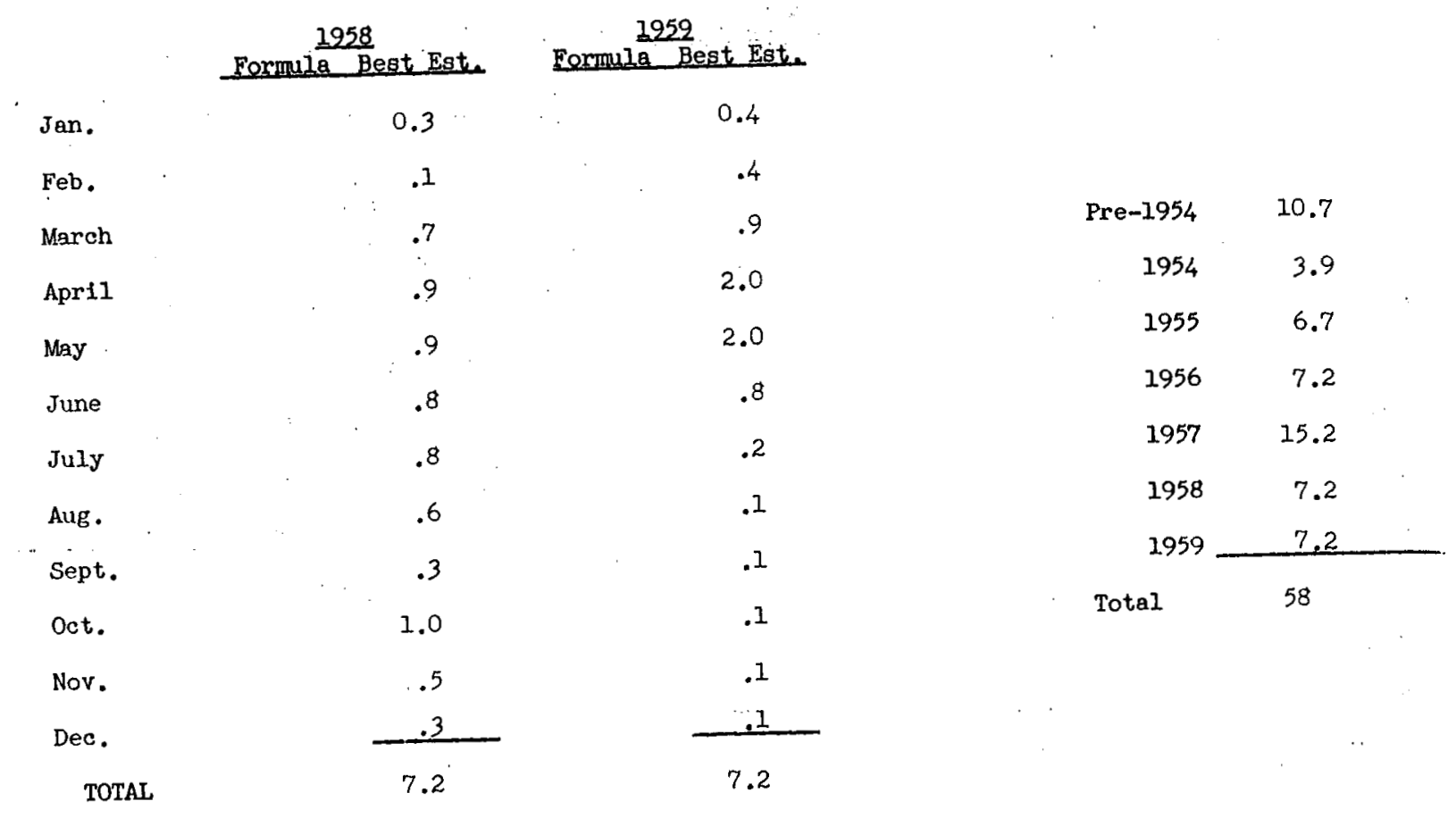


STATTION \# 216

LOCATION INNEAPOLIS, MINNESOTA

1955

Eormula Best Est.

Eormuia Best ist.

2956

1957

Jan.

$0.04 *$

$0.02 *$

Formi-a Best Est

Formula Best Est.

Feb.

.06

.14

Masces

.14

.39

April

.11

.55

May

.07

2.70

1.29

$0.16 *$

0.13

June

.16

$.36 *$

.12

.21

July

.17

$.23 *$

Aug.

.19

$.12 *$

$.18 *$

3.07

Sept.

.21

Oet.

.24

$.16 *$

$.48 *$

.58

Nor.

.19

Dec.

$.03 * *$

1.61

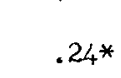

1.24

1.17

.93

.78

.57

1.84

.56

.72

.53

2.07

TOTAL

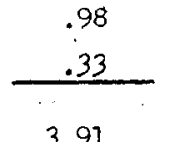

$\begin{array}{r}.48 \\ .18 \\ .15 \\ \hline 5.58\end{array}$

47

.14

.72

8.90

1958

Formula Best Est. Formula Best Est.

\begin{tabular}{lcc} 
Jan. & 0.23 & 0.47 \\
Feb. & .15 & .30 \\
March & .13 & .47 \\
Apri1 & 1.80 & 1.55 \\
May & 1.16 & 2.30 \\
June & .85 & .81 \\
July & .27 & .29 \\
Aug. & .57 & .15 \\
Sept. & .20 & .10 \\
Oct. & .40 & .10 \\
Nov. & .37 & .07 \\
Dec. & -.12 & .06 \\
\hline TOTAL & 6.25 & 6.67
\end{tabular}

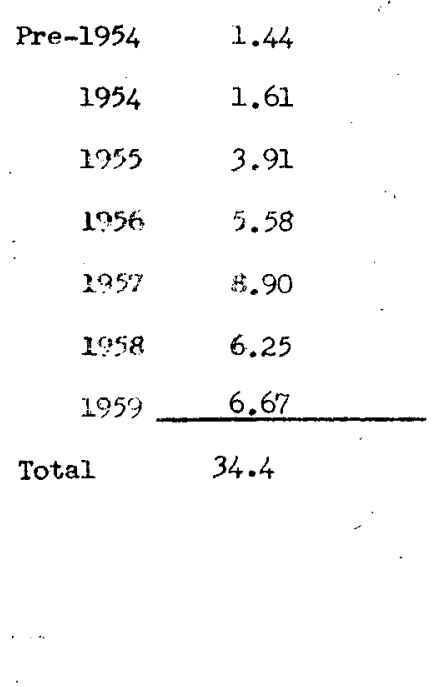




\begin{tabular}{|c|c|c|c|c|c|}
\hline . & Eormuli Best Est. & Eormuta & Best Este & Formule Best Est. & Formila Best Est. \\
\hline Jan. & $0.0 * *$ & & $0.0 *$ & $0.3 *$ & 0.2 \\
\hline Feb. & .1 & & .3 & .1 & .3 \\
\hline March & .8 & & .7 & $.3 *$ & 1.7 \\
\hline Apri1 & .5 & & 1.0 & $.7 *$ & 1.1 \\
\hline May & .3 & 6.6 & 2.5 & 1.9 & 2.0 \\
\hline June & .6 & & $.6 *$ & 1.8 & 1.4 \\
\hline July & .5 & & $.4 *$ & 1.3 & 3.0 \\
\hline Aug. & .5 & & $.2 *$ & 1.2 & 1.1 \\
\hline Sept. & .5 & & $.3 *$ & 1.2 & 1.8 \\
\hline Oct. & .6 & & $.4^{*}$ & .9 & 1.0 \\
\hline Nov. & .4 & . & .1 & .3 & .3 \\
\hline Deo. & $.1 * *$ & & .6 & .3 & 1.3 \\
\hline TOTAL & 4.9 & & 7.1 & 10.3 & 15.2 \\
\hline
\end{tabular}

Formula Beat Est. Formula Best Est.

\begin{tabular}{lcc} 
Jan. & 0.4 & 1.0 \\
Feb. & .2 & .6 \\
March & .3 & .9 \\
Apr11 & 3.1 & 2.8 \\
May & 2.3 & 4.0 \\
June & 1.6 & 1.4 \\
July & .7 & .5 \\
Aug. & 1.5 & .2 \\
Sept. & .5 & .1 \\
Oct. & 1.4 & .1 \\
Nov. & 1.1 & .1 \\
Dec. & -13 & 11.8 \\
Total & 13.4 & .1 \\
\hline
\end{tabular}

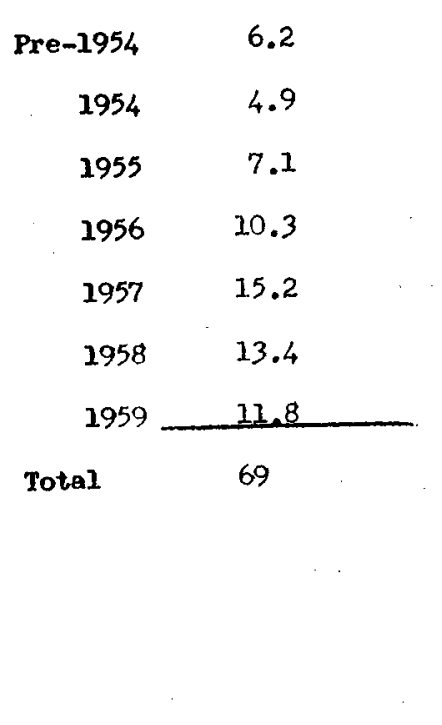


ESITMATE OF Sr 90 DEFOSITION FROM GUMMED EILM

STATION \#_ 219

LOCATION DES MOINES, IOWA

Formula Best Est. $\frac{1254}{1255}$ Formula Best Este Formuia Best Est. Formula Best Est.

Jan.

$0.04 * *$

$0.05 *$

$0.91 *$

0.17

Feb。

.07

.29

March

.16

1.94

0.86

.28

.21

April

.14

1.61

1.02

$.03 *$

.52

May

.07

9.02

1.47

$.03 *$

2.11

June

.13

$.20 *$

JuIy

.16

$.16 *$

Aug.

.22

Sept.

.22

$.12 *$

$.08 *$

Oct.

.27

Nov.

.19

Dec.

$.03 * *$

TOTAL

1.70

$.18 *$

1.34

.99

.33

.81

.62

$1.88 \quad 1.53$

1.13

1.40

.94

1.55

1.26

39

.39

.20

.21

.21

.24

5.04

.12

10.99

Formula Best Est.

0.17

Feb.

18

March

Aprix

31

223

May

.71

June

July

Aug.

Sept.

Det.

Nov.

Dec.

TOTAL $\because 78$

83

.43

$\because 35$

.31

.35

.17

6.82
0.

.48

1.60

2.37

2.81

.44

.17

.15

.09

.05

.04

.09

8.76

\begin{tabular}{rr} 
Pre-1954 & 2.06 \\
1954 & 1.70 \\
1955 & 5.04 \\
1956 & 5.93 \\
1957 & 10.99 \\
1958 & 6.82 \\
1959 & 8.76 \\
\hline Total & 41.3
\end{tabular}


STATION \#_ 219

LOCATION DES MOINES, IOWA

1954 Formula Best Est.

Jan.

Feb

March

Apri1

May

June

July

Aug.

sept.

oct.

Nov .

Deo.

TOTAL
$0.0 * *$

. 1

.8

.8

.3

.5

.5

.6

.6

.7

.6

$1 * *$

5.6

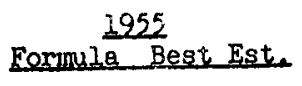

$0.1 *$

.6

3.8

3.0

16.4

1.8

2.6

$.4 *$

$.3 *$

$.2 *$

$.1 *$

$.3 *$

.6

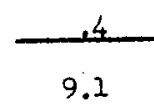

2956

Formile Best Est.

$0.8 *$

.5

$.5 *$

$.5 *$

2.1

.6

1.4

2.5

2.1

.7

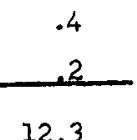

12.3
1957

Eormula Best Est.

0.3

.4

.8

3.8

1.7

1.5

3.12 .5

2.2

2.5

3.2

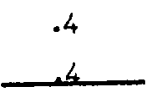

19.7

Formula Best Est.

1259

0.3

Eormule Best Est.

Jan.

.3

Feb.

.6

March

3.8

April

1.4

May

1.5

June

2.2

July

Aug.

1.1

Sept.

.8

oct.

.8

Nov.

1.0

Dec.

TOTAL

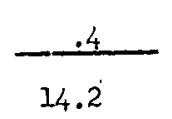

1.0

1.0

3.0

4.3

4.9

.7

.3

.2

.1

.1

Pre-1954 8.5

$1954 \quad 5.6$

$1955 \quad 9.1$

$1956 \quad 12.3$

$1957 \quad 19.7$

$1958 \quad 14.2$

$1959-15.8$

Total

85

.1

.1

15.8 


\section{ESTIMATE OF Sr 90 DEPOSITION FROM GUMMED FILM}

STATION \#_ 221

LOCATION_ST, LOUIS, MISSOURI

1254

1955

1256

Formuls Best Est.

Formula Best Est.

Formile Best Est.

1257

\begin{tabular}{|c|c|c|c|c|c|c|}
\hline Jan. & $0.04 * *$ & & $0.07 *$ & $0.25 *$ & & 0.40 \\
\hline Feb. & .09 & & .44 & .57 & & .66 \\
\hline March & .09 & 6.09 & 1.46 & $.72 *$ & & 1.08 \\
\hline April & .10 & 2.55 & 1.77 & $.61 *$ & & 1.73 \\
\hline May & .14 & 11.14 & 2.15 & 1.36 & & 1.25 \\
\hline June & .25 & & $.34^{*}$ & .34 & & 1.21 \\
\hline July & נj. & & $.23 *$ & .80 & 3.22 & 2.05 \\
\hline Aug. & .30 & & $.10 *$ & .47 & & $.71 * *$ \\
\hline Sept. & .15 & & $.10 *$ & .92 & & 1.41 \\
\hline Oct. & .26 & & $.33 *$ & .33 & & .71 \\
\hline Nov. & .27 & & .37 & .24 & & .25 \\
\hline Dec. & $.03 * *$ & & .24 & .23 & & .57 \\
\hline TOTAL & 1.91 & & $7.60^{\circ}$ & 6.84 & & 12.03 \\
\hline
\end{tabular}

1959

Formula Best Este Formula Best Est.

$\begin{array}{lcc}\text { Jan. } & 0.51 & 0.57 \\ \text { Feb. } & .67 & .91 \\ \text { March } & 1.48 & 2.14 \\ \text { April } & 2.69 & 2.03 \\ \text { May } & .86 & 2.52 \\ \text { June } & .99 & 0.27 \\ \text { July } & 1.29 & .24 \\ \text { Aug. } & .46 & .15 \\ \text { Sept. } & .21 & .09 \\ \text { Oct. } & .30 & .08 \\ \text { Nov. } & .65 & .02 \\ \text { Dec. } & -.45 & .09 \\ \text { TOTAL } & 10.56 & 9.11\end{array}$




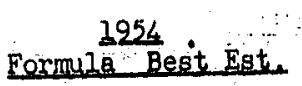

$0.0 * *$

Jan.

Feb.

March

Apri1

May

June

July

Aug .

Sept.

oct.

Nov.

Dec.

TOTAL
.2

.5

.6

.6

.9

.6

.9

.4

.6

.6

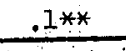

6.0
Cormuls Best Este

$0.1 *$

$11.8 \quad 2.8^{2}$

$4.7 \quad 3.3$

$20.3 \quad 3.9$

$.6 *$

$.4^{*}$

$.2 *$

$.2 *$

$.5 *$

.6

.4

13.9
2956

Cormili Best Est.

$0.4 *$

$.9 *$

$1.1 *$

.9

2.3

.7

1.8

1.0

2.0

.6

.5

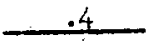

12.6
1952

Eormula Best Est.

0.7

1.1

1.7

3.0

2.1

2.2

$5.2 \quad 3.3$

$1.1 * *$

2.2

1.6

.5

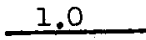

20.3
Jan.

0.9

Feb.

March

Apri1

May

June

July

Aug.

Sept.

Oct.

Nov.

Dec.

TOTAL
1.6

2.9

4.6

1.7

1.8

3.4

1.2

.5

1.0

1.9

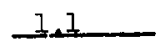

22.6
1.2

1.8

4.1

3.7

$4 \cdot 4$

.5

.4

.2

.1

.1

.0

.1

16.6

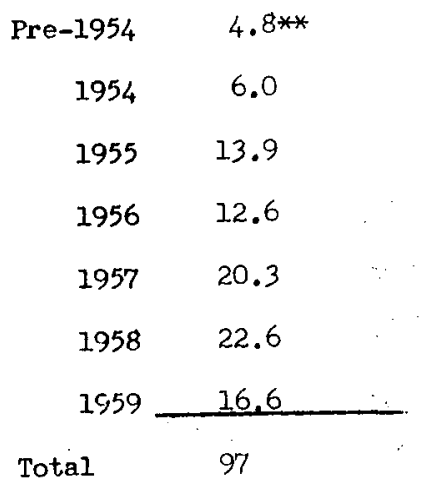




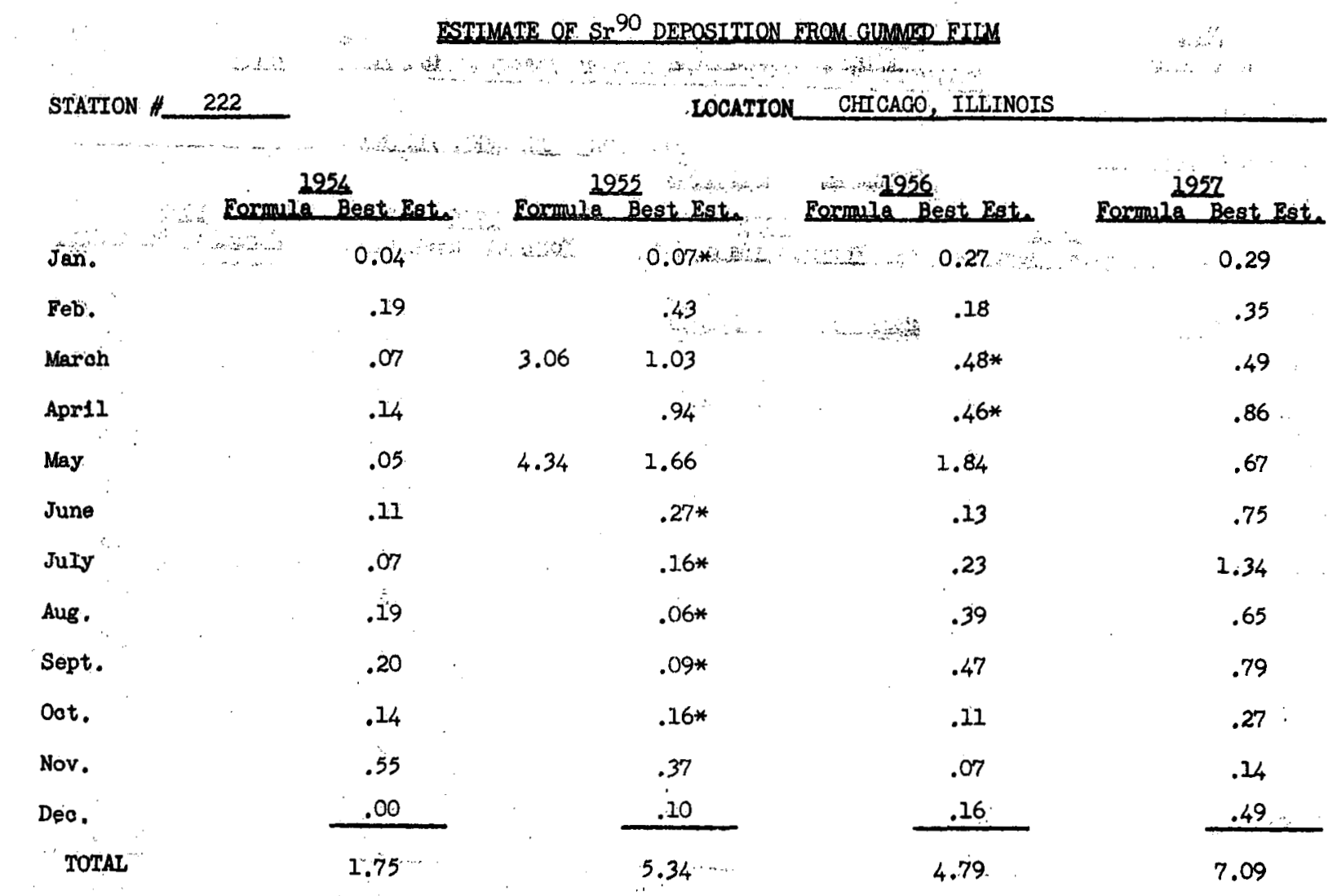


STATION \# 222

LOCATION CHLCAGO. IITINOIS

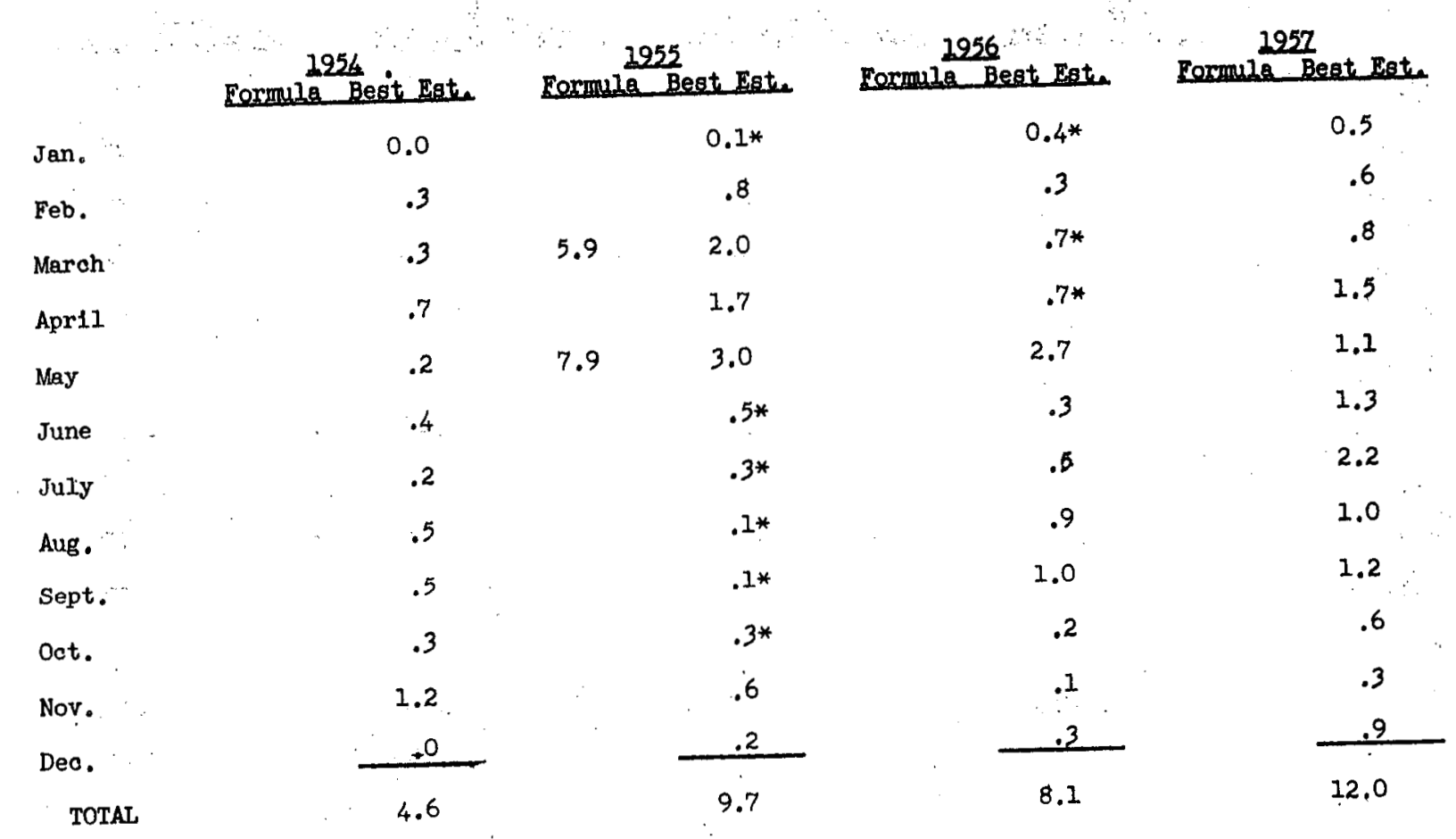

Formula Best Este.

Jan.

0.3

Feb.

March

Apri1

May

June

July

Aug.

Sept.

oct.

Nov.

Dec.

TOTAL $1959^{\circ}$

Formila Best Est.

0.8

1.5

6.4

4.7

3.5

.9

.3

.1

.1

.1

\begin{tabular}{rl} 
Pre-1954 & 7.7 \\
1954 & 4.6 \\
1955 & 9.7 \\
1956 & 8.1 \\
1957 & 12.0 \\
1958 & 18.0 \\
1959 & 18.6 \\
\hline Total & 79
\end{tabular}

.1

.1

18.6 


\section{ESTIMATE OE Sr90 DEPOSITTON FROM GLMMED ELLM}

STATrON \# 225

LOCATION NEW ORLEANS, LOUISIANA

1954

\section{Eormule Best Este.}

0.04

Jan.

Feb.

March

April

May

June

July

Aug .

Sept.

Oot.

Nov.

Deo.

TOTAL

\begin{tabular}{l}
.08 \\
.18 \\
.23 \\
.16 \\
.19 \\
.23 \\
.12 \\
.22 \\
.11 \\
.23 \\
.03 \\
\hline 1.82 \\
\hline
\end{tabular}

2955

Eormula Best Este.

$0.05 *$

.17

.49

1.10

7.40

2.87

$.29 *$

$.34 *$

$.24 *$

$.15 *$

$.10 *$

.21

.22

6.23
2956

Cormula Best Est.

$0.39 *$

.46

$.38 *$

$.20 *$

.63

.47

1.25

.57

.34

.24

.19

25

5.37
2957

Eormila Best Rste

0.35

.40

.74

.94

.45

$3.75 \quad 1.52$

1.37

.82

.90

.41

.24

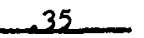

8.49

958

Formula Best Estic Eormula Begt Este

Jan.

Feb.

March

Apr11

May

June

July

Aug.

Sept.

Oct.

Nov.

Dec.

TOTAL
0.38

.53

.55

.71

.70

38

.82

... .82

.52

.26

.21

.17

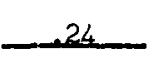

5.47
0.76

1.18

3.13

.89

.87

.45

.12

.07

.08

.06

.06

.01

7.68
Pre-1954 $\quad 7.44$

$1954 \quad 1.82$

$1955 \quad 6.23$

$1956 \quad 5.37$

$1957 \quad 8.49$

$1958 \quad 5.47$

$1959+7.68$

Total

36.5 


\section{ESTIMATE OF INFINITY $\gamma$ DOSE FROM GUMMD FILM}

STATION \#-225

LOCATION NEW ORLEANS, LOUISIANA

1954,1955 Eormule Best Est.

Jan.

Feb.

March

Apr1?

May

June

JuTy

Aug.

Sept.

oot.

Nov:

Deo.

TOTAL
0.0

.1

1.0

1.3

.9

.7

.7

.4

.. .6

.3

.5

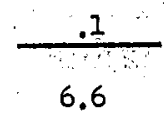

Formule Best Est.

$0.1 *$

.3

.9

2.0

13.4

5.2

$.5 *$

$.6 *$

$.4 *$

$.2 *$

$.2 *$

.3

.4

11.1
Formule Best Est.

$0.6 *$

.7

$.6 *$

$.3 *$

1.0

.9

2.8

1.3

.8

.4

.4

.5

10.3
2957

Formule Best Est.

0.6

.7

1.2

1.6

.8

$6.7 \quad 2.8$

2.2

1.3

1.5

.9

.5

.6

14.7

1958

Formula Best Est. Formule Best Est.

Jan.

Feb.

March

Apri1

May

June

July

Aug.

Sept.

Oct.

Nov.

Dec.

TOTAL
0.6

.9

1.1

1.2

1.4

.8

2.2

1.4

.6

.7

.5

6

12.0
1.6

2.4

6.0

1.6 .

1.5

.8

.2

.1

.1

.1

\begin{tabular}{rl} 
Pre-1954 & 8.0 \\
1954 & 6.6 \\
1955 & 11.1 \\
1956 & 10.3 \\
1957. & 14.7 \\
1958 & 12.0 \\
1959 & 14.5 \\
\hline Total & 77
\end{tabular}




\section{ESTIMATE OF Sr 90 DEPOSITION FROM GUMMED FILM}

STATION \# 304

LOCATION BOISE, IDAHO

1254 2955

1956

1957

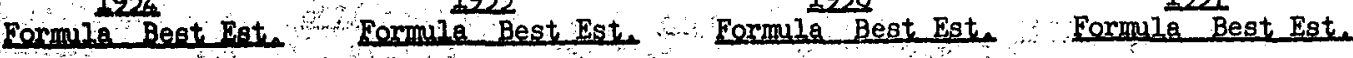

\begin{tabular}{|c|c|c|c|c|c|c|}
\hline Jan. & 0.04 & & $0.05 *$ & $0.37 *$ & & 0.21 \\
\hline Feb. & .09 & & .25 & .30 & & .40 \\
\hline March & .03 & & .35 & $.26 *$ & & .80 \\
\hline Apr11 & .03 & & 1.28 & $.87 *$ & & .84 \\
\hline May & .43 & 5.82 & 1.88 & .87 & & 1.10 \\
\hline June & .11 & & $.25 *$ & .38 & & .62 \\
\hline JuIy & .10 & & $.14 *$ & .58 & 3.54 & 0.85 \\
\hline Aug. & .11 & & $.07 *$ & .24 & & .36 \\
\hline Sept. & .21 & & $.05 *$ & .23 & 1.73 & 1.31 \\
\hline Oat. & .08 & & $.25 *$ & .44 & & 1.13 \\
\hline Nov. & .09 & & .15 & .12 & & .16 \\
\hline Deo. & .03 & $\therefore$ & .53 & $\ldots 18$ & & 47 \\
\hline TOTAL & 1.25 & & 5.05 & 4.84 & & 8.25 \\
\hline
\end{tabular}

TOTAL

8.25

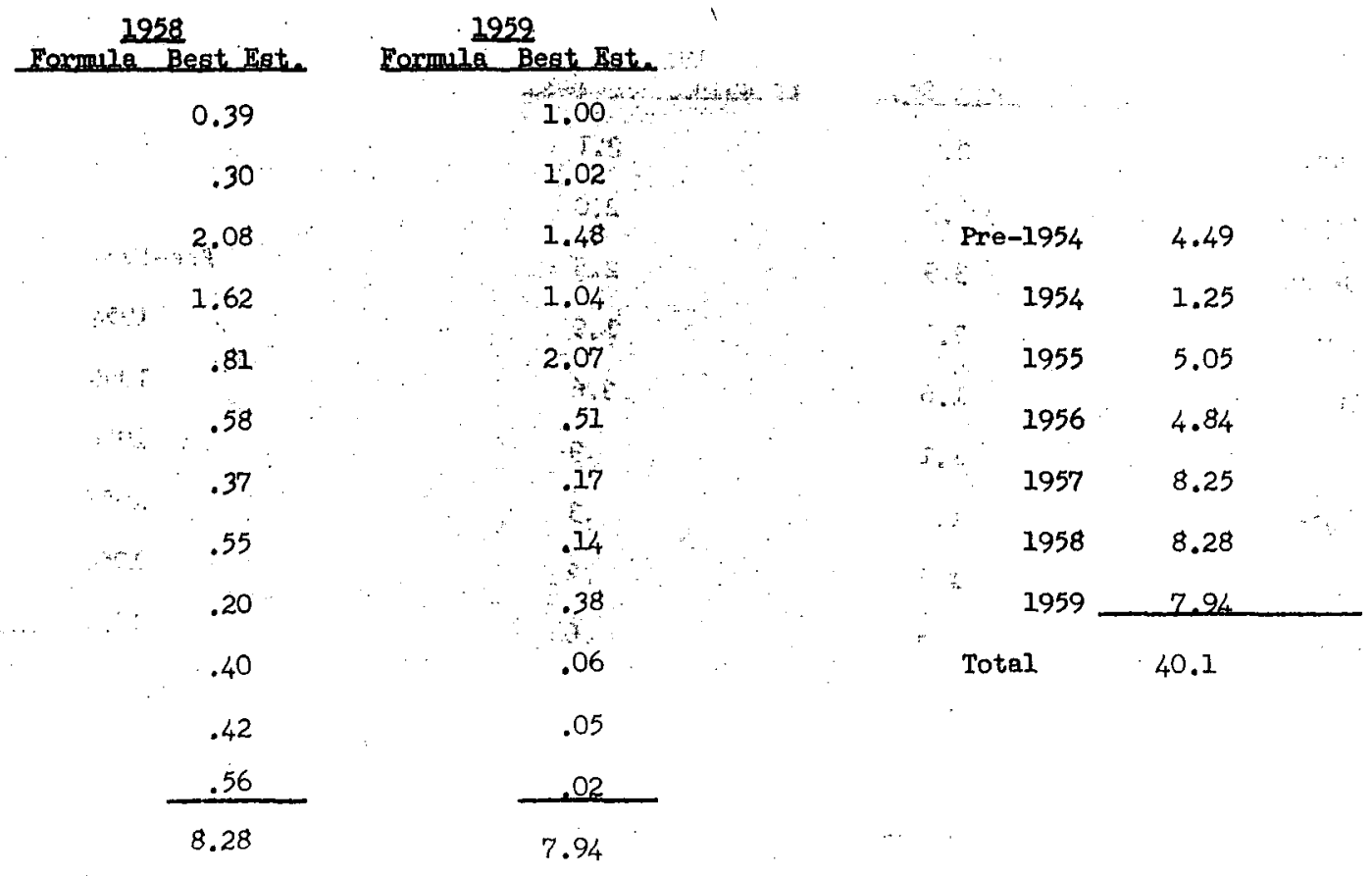


STATION $\quad 304$

सh, 1954 Eormula Best Eat.

Jan.

0.0

Feb.

Maroh

ApriI

Mey

June

JuIy

Aug.

Sopt.

oot.

Nov.

Deo.

TOTAT
.1

.2

.1

2.7

.4

.3

.3

.3

.2

.2

.1

4.9

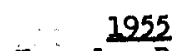

Conmula Besti Egta

$0.1 *$

.3

.7

2.4

10.5

3.4

$.4 *$

$.2 *$

:I*

$.1 *$

$.2 *$

.2

.9

9.0
1956

Formula Best Est

$0.6 *$

.5

$.4 *$

1.3*

1.3

.7

1.3

.5

.5

.8

.2

.3

8.4
2957.

Pormila Best Bst.

0.4

.7

1.3

1.6

1.9

1.2

1.4

.6

$3.5 \quad 2.4$

2.2

.3

.8

14.8
Jen.

Feb

March

Apri1

May

June

July

Aug.

Sept.

oct.

Nov:

Dec.

TOTAL
0.7

.5

3.9

2.8

1.6

1.1

1.0

1.4

.5

1.6

1.2

1.3

17.6
Formula Best Eat.

$$
2.1
$$

2.0

2.8

1.9

3.6

.9

.3

.2

.6

.1

Pre-1954 $\quad 3.6$.

$1954 \quad 4.9$

19559.0

$1956 \quad 8.4$

$1957 \quad 14.8$

$1958 \quad 17.6$

$1959-14.6$

Total.

73 
ESTIMATE OF SF 90 DEPOSITION FROM GUMMED FILM

STATION \#309

LOCATION BILLINGS, MONTANA

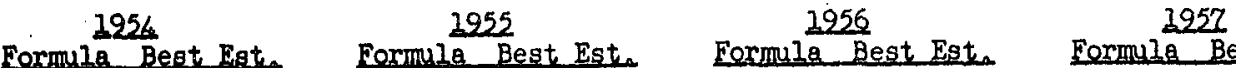

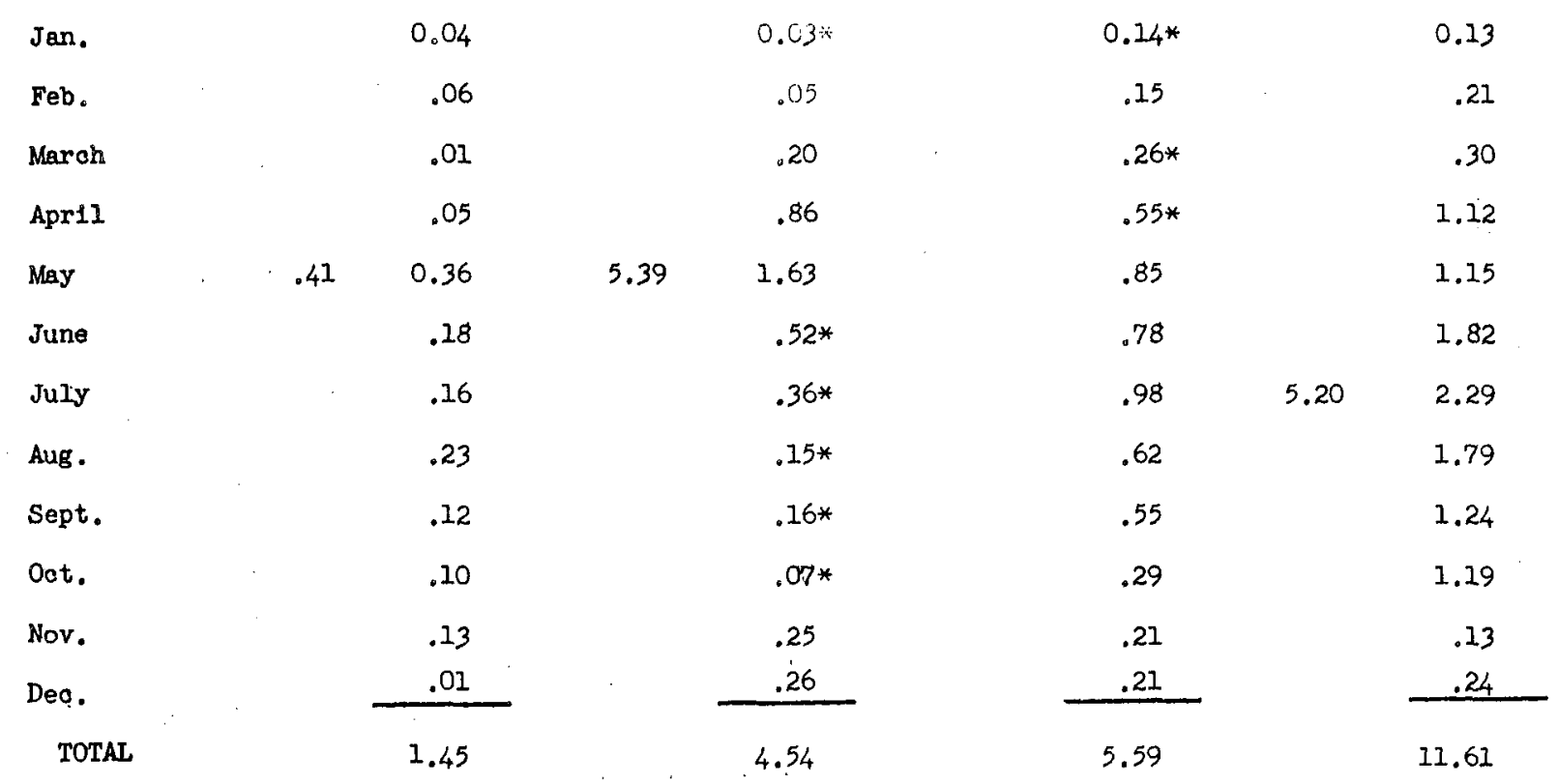

1928

Formula Best Eat.

Jan.

0.16

Feb.

March

Apri1

May

June

July

Aug.

Sept.

oct.

Nov.

Dec.

TOTAL
.10

.39

1.59

1.05

.94

.43

.47

.35

.83

.48

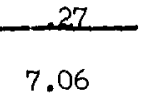

2959

Formula Best Est.

0.41

.22

.80

1.27

2.40

1. 07

.36

.21

.08

.08

Pre-1954 $\quad 1.61$

$1954 \quad 1.45$

$1955 \quad 4.54$

$1956 \quad 5.59$

$1957 \quad 11.61$

$1958 \quad 7.06$

1959

Total

38.9

.06

-

6.99 
195402955

Cormule Best Eat.

Feb.

Maroh

Apr11

May

June

July

Aug.

Sept.

oct.

Nov.

Dec.

TOTAL

$$
0.0
$$

.1

.0

.2

$2.5 \quad 1.9$

.7

.5

.5

.3

.2

.3

.0

4.9
Eormile Begt Esto

$0.1 *$

.1

.4

1.6

$9.8 \quad 2.9$

$.9 *$

$.6 *$

$.6 *$

$.3 *$

$.1 *$

.4

.4

8.1
1956 Formula Best Est.

$0.2 *$

.2

$.4 *$

$.8 *$

1.3

1.5

2.3

2.3

1.2

.6

.4

.4

10.7
1957

Eormula Best Esto

0.2

.3

.5

2.1

1.9

3.4

$8.3 \quad 3.6$

2.9

2.0

2.4

.3

.4

20.0

1958 Formula Best Est.

1959

0.3

Formiz Best Est.

Jan.

.2

Feb.

.7

March

2.7

Apr 11

2.0

May

1.8

June

1.1

July

Aug.

1.2

Sept.

.8

oct.

2.8

Nov.

1.5

Dec.

TOTAL

0.9

.4

1.5

2.3

4.2

1.8

.6

.3

.1

.1

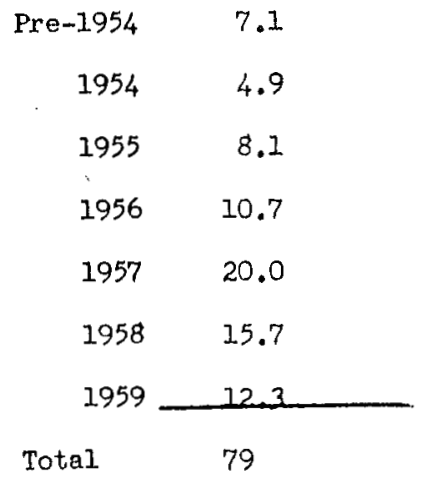

.1

.0

12.3 
rOCATIOA SALT LAKE CITY, UTAH

1956 1955 1956

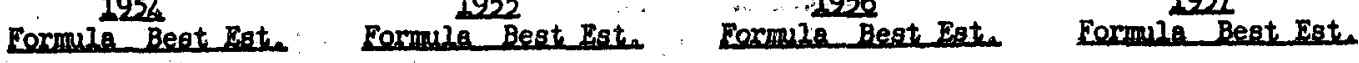

Jan.

$0.04 * *$

Feb.

Maroh

April

May

June

July

Aug .

Sept.

oat.

Nov.

Deo.

TOTAL
.05

.02

.14

.60

.23

.21

.20

.21

.25

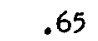

$.01 * *$

2.61
$0.03 *$

4.25

6.78

44.25

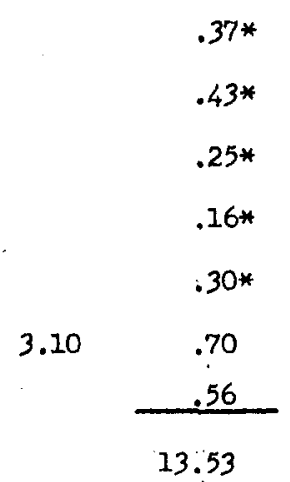

$0.62 *$

.29

$.14^{*}$

$.67 *$

1.30

.33

.79

.49

.64

.80

.30

26

6.63
0.47

.59

1.53

1.77

1.49

2.07

2.40

4.63

2.06

2.66

1.88

1.77

.31

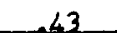

16.77

Formula Best Eat.

1952

Formula Best Eat.

Jan.

0.39

1.27

Feb.

.49

1.24

March

2.26

1.21

April

3.69

2.64

May

1.69

3.77

June

.75

July

.59

Aug.

.57

Sept.

oct.

.24

.38

Nov.

.42

1.44

.41

.25

$\therefore 20$

.14

Pre-1954 9.64

$1954 \quad 2.61$

$1955 \quad 13.53$

$1956 \quad 6.63$

$1957 \quad 16.77$

$1958 \quad 12.42$

1959

12.82

Total

74.4

Dec.

.95

.15

.10

TOTAL

12.42

12.82 
$\therefore$ a

ESTIMATE OF INFINITY y DOSE FROM GMMAD FILM

STATTON $\quad 310$

LOCATION SALT LAKE CITY, UTAH

1955

Eormula Begt Esta

$0.1 *$

Jan,

$0.0 *$

Feb.

Merch

Apr11

May

June

July

Aug.

Sept.

oct.

Nov.

Dec.

TOTAL
.1

.1

.7

3.3

.9

$\therefore .7$

.6

.5

.6

.1 .4

$.0 *$

8.9
.2

8.23 .2

12.6

3.1

80.4

13.3

$.6 *$

$.7 *$

$.4 *$

$.3 *$

$.5 *$

5.0

1.1

1.0

24.5
1956 Formula Best Est. $1.0 *$

.5

$.2 *$

$1.0 *$

2.1

.6

1.8

1.1

1.4

1.5

.6

.5

12.3
1957.

Eormula Best Eate.

0.8

1.0

2.5

3.3

2.6

3.8

3.9

$7.7 \quad 3.2$

$4.2 \quad 2.9$

3.5

.7

.8

29.0

1958

Formutie Best Est.

Jan.

0.7

Feb.

.8

March

4.4

Apr 11

6.4

May

3.3

June

1.4

July

1.5

Aug.

1.5

Sept.

.6

Oct.

1.6

Nov.

1.3

Dec.

TOTAL

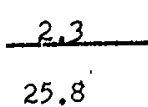

Formula Best Est.

$$
2.7
$$

2.5

2.3

4.7

6.6

2.4

.7

.4

.3

.2

\begin{tabular}{rr} 
Pre-1954 & 34.7 \\
1954 & 8.9 \\
1955 & 24.5 \\
1956 & 12.3 \\
1957 & 29.0 \\
1958 & 25.8 \\
1959 & 23.2 \\
\hline Total & 158
\end{tabular}

.2

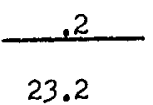


STATION \# 314

LOCATION TUCSON, APIZONA

1254

Formula Begt Est:

1955

Tormula Best Est.

1956

Formule Best Est.

1957

0.04

$0.03 *$

$0.27 *$

0.33

Feb.

.06

.23

.28

.23

March

.17

1.29

$.69 *$

.33

April

.29

.22

$.22 *$

.32

May

.80

.59

.16

.62

June

.18

July

.40

$.24 *$

.49

1.73

1.01

Aug.

.19*

1.56

23.67

3.82

$.13 *$

.53

1.42

Sept.

.10

oct.

.05

Nov.

.04

Deo.

.00

.05*

.32

.51

.23*

.24

.63

$.58 * *$

.20

.46

.11

.49

TOTAL

2.24

.03

5.07

10.17

3.71

Formula Best Est. Eormula Best Esit.

Jan.

Feb.

0.14

.28

March

Apr11

May

June

July

Aug.

Sept.

Oct.

Nov.

Dec.

TOTAL
1.31

1.65

.52

.55

1.48

.74

.25

.33

.57

.08

7.90
0.31

.30

.37

.98

.32

.55

.34

.03

.10

.10

.06

.06

3.52

\begin{tabular}{rr} 
Pre-1954 & 1.17 \\
1954 & 2.24 \\
1955 & 3.71 \\
1956 & 5.07 \\
1957 & 10.17 \\
1958 & 7.90 \\
1959 & 3.52 \\
\hline Total & 33.8
\end{tabular}


IOCATION_TUCSON, ARIZONA

\begin{tabular}{|c|c|c|c|c|c|}
\hline$\therefore \quad$. & Formula Besit Est. & $\frac{1955}{\text { Eormula Best Este. }}$ & $\frac{1956}{\text { Formula Best Este }}$ & Formula & Best Est. \\
\hline Jan. & 0.0 & $0.1 * *$ & $0.4^{*}$ & . & 0.6 \\
\hline Feb. & .1 & .4 & .5 & & .4 \\
\hline March & 1.0 & 2.4 & $1.0 *$ & & .5 \\
\hline Apr11 & 1.5 & .4 & $.3 *$ & & .6 \\
\hline May & 4.6 & 1.1 & .3 & & 1.0 \\
\hline June & .7 & $.2 *$ & .9 & 3.1 & 1.8 \\
\hline July & 1.3 & $.3^{*}$ & 3.8 & 22.7 & 5.8 \\
\hline Aug. & .3 & $.2 *$ & 1.2 & & 2.2 \\
\hline Sept. & .3 & $.1 *$ & .7 & & .9 \\
\hline Oct. & .1 & $\quad .4 *$ & .5 & & 1.3 \\
\hline Nov. & .1 & $1.0 * *$ & .4 & & 1.0 \\
\hline Deo. & .0 & .1 & - & & 0.9 \\
\hline TOTAL & 10.0 & 6.7 & 10.2 & & 17.0 \\
\hline
\end{tabular}

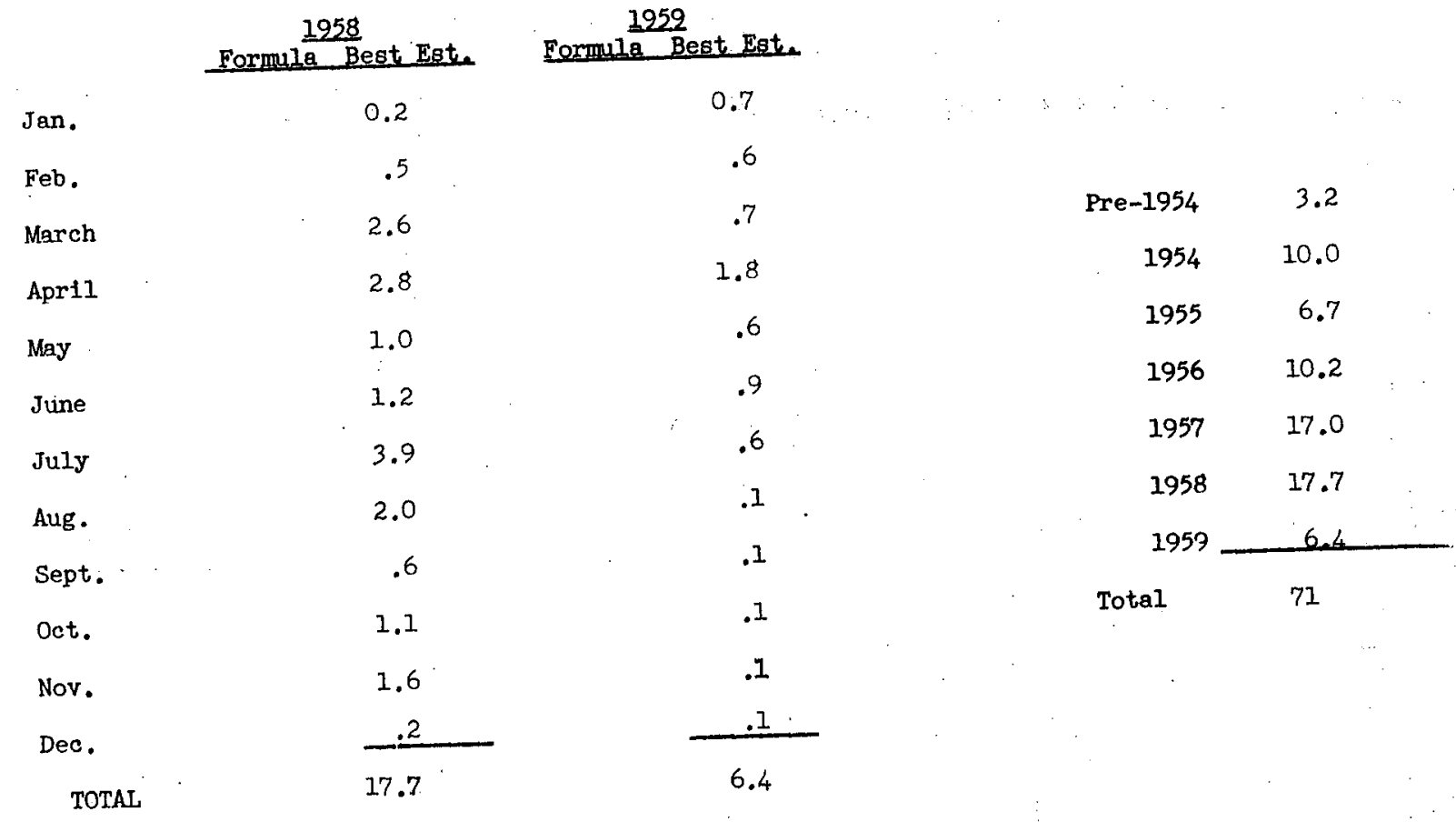


ESTIMATE OF Sr 90 DEPOSITION FROM GUMMD FIU

STATION \# 321

LOCAMION GRAND JUNCTION, COLORADO

.. 2254

1956

ua Best Est.

JE.1.

0.04

$0.04 *$

0.19*

0.36

Feb.

.09

.06

.44

.21

March

.08

4.70

2.88

$.26 *$

.86

ApriI

.19

$11.37 \quad 5.80$

$.84 *$

1.36

May

.45

$9.13 \quad 1.84$

‥ $\quad .90$

1.19

June

.20

$.35 *$

.49

1.23

July

.16

Aug.

.22

$.19 *$

1.06

$2.68 \quad 1.78$

Sept.

.24

$.09 *$

$.09 *$

Oct.

.15

$.09 *$

.37

.66

1.49

Nov.

.43

1.94

.45

Dec.

.00

TOTAL

2.24

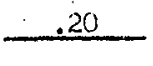

12.08

.32

1.16

$.25 * *$

.14

23

10.78

1958

1259

Formula Best Est. Formula Best Est.

Jan.

0.32

Feb.

March

April

May

June

July

Aug.

Sept.

Oct.

Nov.

Dec.

TOTAL
.18

1.68

1.45

1.22

.83

2.02

.31

.40

.24

.52

$-.25$

8.42

0.23

.70

.90

2.19

2.54

.91

.29

.13

.14

.08

\begin{tabular}{rr} 
Pre-1954 & 5.24 \\
1954 & 2.24 \\
1955 & 12.08 \\
1956 & 5.54 \\
1957 & 10.78 \\
1958 & 8.42 \\
1959 & 8.20 \\
\hline Total & 52.5
\end{tabular}

.04

.05

8.20 
STATION \# 321

LOCATION GRAND JUNCTION, COLORADO

\begin{tabular}{|c|c|c|c|c|c|}
\hline & Eormula Best Este & Eormpla & est Est. & 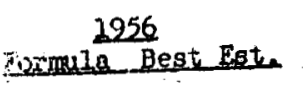 & 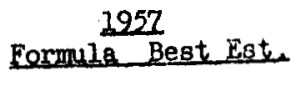 \\
\hline , & 9.3 & & $0.1^{*}$ & $0.3 *$ & 0.5 \\
\hline Fot & $\therefore$ & & .1 & .7 & .4 \\
\hline March & .5 & 9.0 & 5.7 & $.4^{*}$ & 1.4 \\
\hline Apr11 & 1.0 & 21.1 & 10.8 & $1.2 *$ & 2.4 \\
\hline May & 2.5 & 16.5 & 5.0 & 1.4 & 2.0 \\
\hline June & .8 & & $.6 *$ & 1.0 & 2.2 \\
\hline Juty & .5 & & $.3 *$ & 2.4 & 4.4 \\
\hline Aug . & .6 & & $.2^{*}$ & .8 & 1.0 \\
\hline Sept. & .6 & & $.2 *$ & .7 & 2.3 \\
\hline oct. & .4 & & $.1 *$ & .7 & 2.4 \\
\hline Nov, & 1.0 & 3.1 & .8 & .3 & $.5 * *$ \\
\hline Dec. & .0 & & .3 & .3 & .4 \\
\hline TOTAL & 8.0 & & 24.2 & 10.2 & 18.3 \\
\hline
\end{tabular}

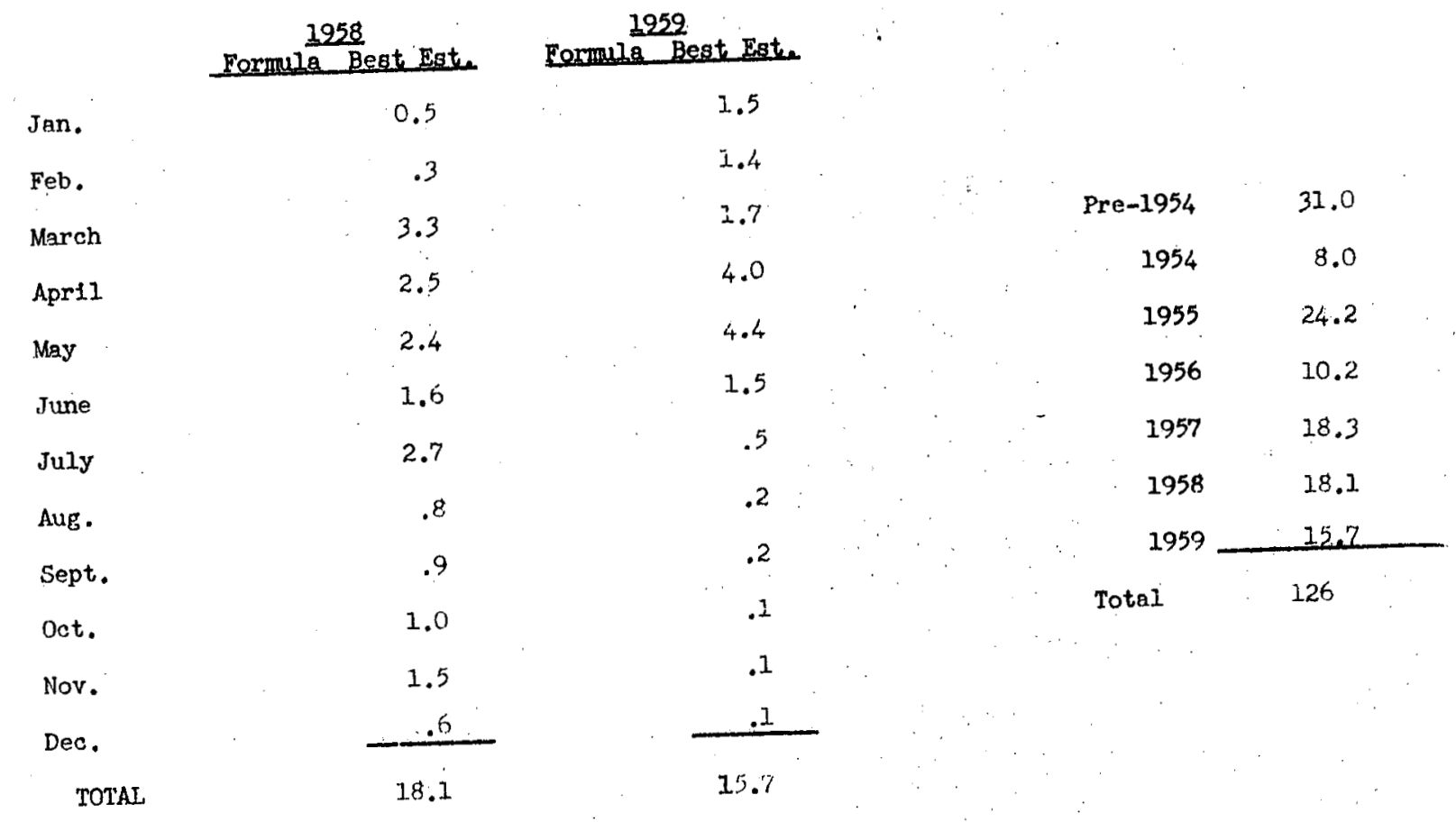




\section{ESTIMATE OF Sr 90 DEPQSITION FROM GUMMED EILW}

STATION \# 323

LOCATION_ ALBUQUERQUE, NEW MEXICO

\section{4}

Formula Best Este Pormula Hest Est.

$$
0.34 *
$$

1957

Jan.

$0.04 * *$

$0.03 *$

.37

0.23

Feb,

.25

$1.07 \quad .18$

.48

$3.78 \quad .62$

April

.22

May

June

.22

.85

$4.99 \quad, 71$

$.06 *$

$.12 *$

$.12 *$

$.52 *$

JuIy

.24

Aug.

.24

$.26 *$

.16

$.28 *$

Sept.

.14

$.22 *$

oet.

.11

$.19 *$

Nov.

.61

.60

Dec.

TOTAL
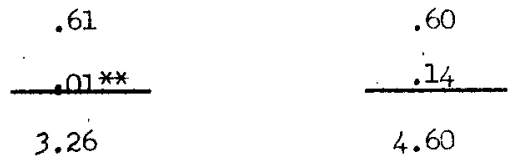

1.37

1.77

$2.11 \quad 1.34$

20

$4.62 \quad 2.64$

.55

.78

.25

$3.17 \quad 1.29$

.30

.96

.24

.30

.08

.47

6.12

11.70

Formula Best Est. Formula Best Est.

Jan.

0.25

0.26

Feb.

.35

.29

March

.83

.93

Apri1

2.45

May

1.29

June

.99

July

1.22

Aug.

.84

Sept.

.27

Oct.

.35

Nov.

Dec.

TOTAL

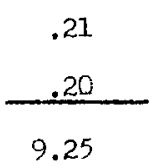

2.16

2.15

.85

.45

.15

.11

.12

$$
\begin{array}{rr}
\text { Pre-1954 } & 9.09 \\
1954 & 3.26 \\
1955 & 4.60 \\
1956 & 6.12 \\
1957 & 11.70 \\
1958 & 9.25 \\
1959 & 7.61 \\
\hline
\end{array}
$$

Total

51.6

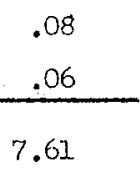


STATION \#_323

LOCATION ALBUQUURQUE, NEW NEXICO

$1254.2955,1956$ arormil Best Este Eormule Best Est 4 Formule Best Est.

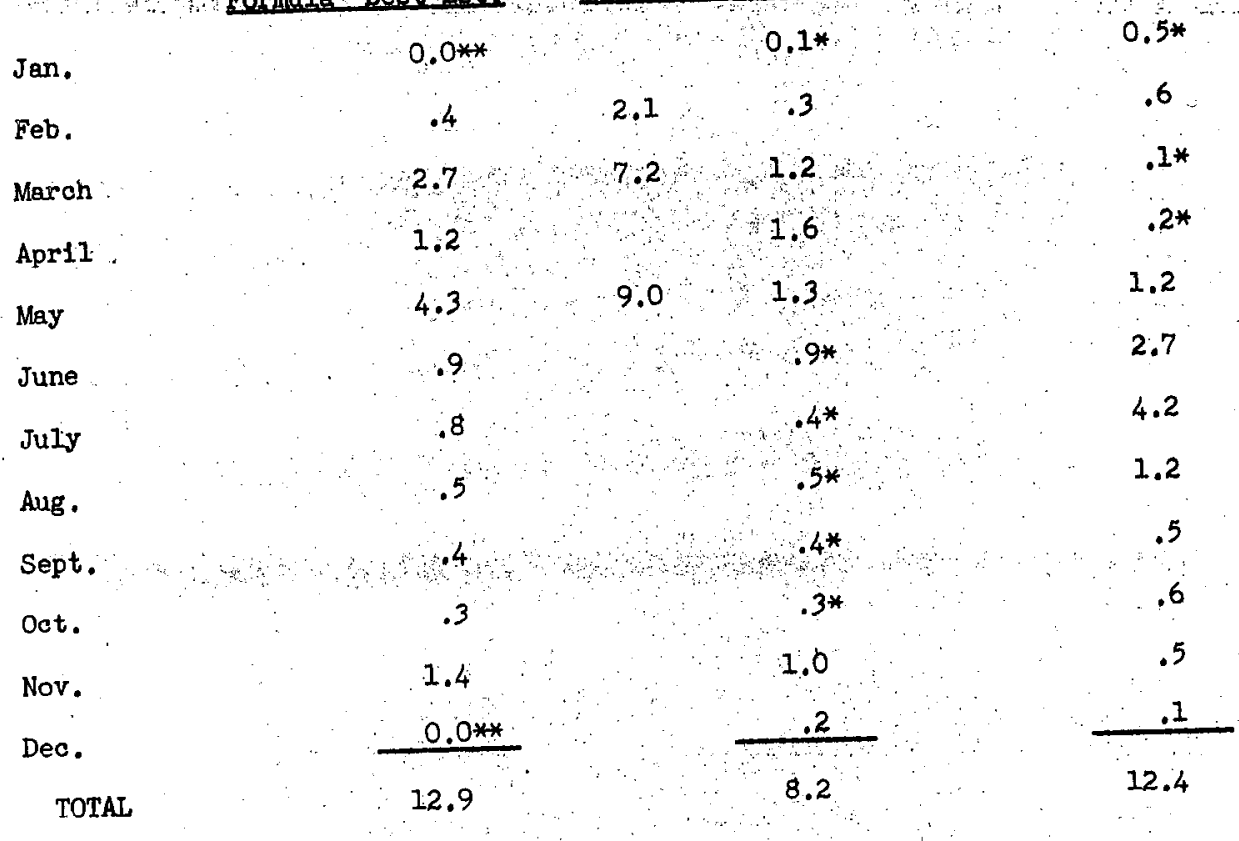

Bormule Best ant.

0.4

.4

1.3

1.6

2.8

2.4

4.3

1.2

2.1

1.9

.6

.9

19.9

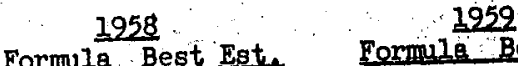

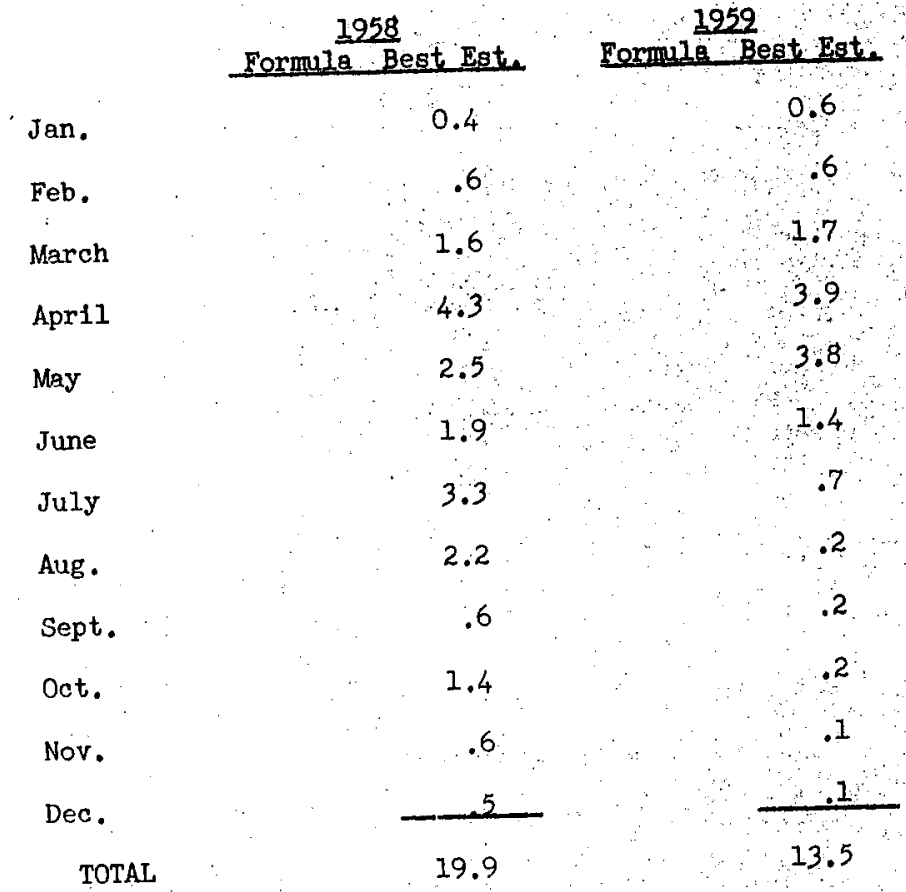

\begin{tabular}{rr} 
Pre-1954 & 54.3 \\
1954 & 12.9 \\
1955 & 8.2 \\
1956 & 12.4 \\
1957 & 19.9 \\
1958 & 19.9 \\
1959 & 13.5 \\
\hline Total & 141
\end{tabular}


STATION \#. 326

1954

Bormula Best Bst.

Jain.

Fèb.

Maroh

April

Mäy

June

JuIy

Aing.

Sept.

oot.

Nov.

Deo.

TOral
0.00

.03

.01

.04

.30

.09

.12

.10

.03

.17

.43

.02

1.34

IOCATION LAS VEGAS, NEVADA

1955

Eormula Best Est.

$0.05 *$

.20

5.48

1.65

2.20

0.90

1.31

$.25 *$

$.03 *$

$.08 *$

$.20 *$

$.17 *$

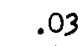

.18

5.05
2956

Eoxmula Best Est.

$0.35 *$

.05

$.31 *$

$.59 *$

.45

.22

.60

.09

.02

.31

.11

.13

3.23
2957

Formula Best Est.

0.33

.12

.32

.37

.57

.37

1.04

.15

.55

.22

.13

.34

4.51

Formula Best Est. Bormila Best Est.

Jan.

0.37

0.29

Feb.

March

.21

.67

Apr11

.74

.65

1.27

.64

May

.50

June.

.16

July

.51

1.36

.46

.15

Aug.

.46

.09

Sept.

.26

.10

oct.

1.00

.15

Pre-1954 $\quad 5.88$

Nov.

.72

.07

Dec.

.25

TOTAL

6.35

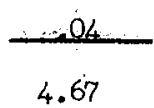

$1955 \quad 5.05$

$1956 \quad 3.23$

$1957 \quad 4.51$

$1958 \quad 6.35$

1959

Total.

31.0 
STATION \#

1954

Formula Besit Est.

$\begin{array}{lc}\text { Jan. } & 0.0 \\ \text { Feb. } & .0 \\ \text { March } & .0 \\ \text { Apr11 } & .2 \\ \text { May } & 1.6 \\ \text { June } & .3 \\ \text { July } & .4 \\ \text { Aug. } & .3 \\ \text { Sept. } & .1 \\ \text { Oct. } & 1.0 \\ \text { Nov. } & .1 \\ \text { Dec. } & 4.4 \\ \text { Total } & \end{array}$
LOCATION IAS VEGAS, NEVÉADA

$1955 \quad 2956 \quad 2957$

Eormule Best Este.

$0.1 *$

10.3

.4

4.1

3.2

1.4

2.4

$.4 *$

$.1 *$

$.1 *$

.3*

.3*

.0

.3

9.0
.1

$.5 *$

$.9 *$

.7

.4

1.5

.2

.0

.6

.2

3

6.0
Formula Best Est.
Formula Best Eet.

0.6

.2

.5

.7

1.0

.7

1.7

.2

1.0

.5

.3

.6.

8.0

1958 1959

Formula Best Eat.

$\begin{array}{lcc}\text { Jan. } & 0.4 & 0.6 \\ \text { Feb., } & .4 & 1.3 \\ \text { March } & 1.5 & 1.2 \\ \text { April } & 2.2 & 1.2 \\ \text { May } & 1.0 & 2.4 \\ \text { June } & .3 & .8 \\ \text { July } & 1.3 & .2 \\ \text { Aug. } & 1.3 & .1 \\ \text { Sept. } & .6 & .2 \\ \text { Oct. } & 4.0 & .2 \\ \text { Nov. } & 2.1 & .1 \\ \text { Dec. } & -.4 & .1 \\ \text { TOTAL } & 15.5 & 8.4 \\ & & \end{array}$

$\begin{array}{rc}\text { Pre-1954 } & 23.7 \\ 1954 & 4.4 \\ 1955 & 9.0 \\ 1956 & 6.0 \\ 1957 & 8.0 \\ 1958 & 15.5 \\ 1959 & 8.4 \\ \text { Total } & 75\end{array}$


ESTIMATE OF Sr 90: DEPOSITLON FHOM GUMMED FIIM

STATION \#_ 401

LOCATION__ SEATTLE, WASHINGTON

Formula Best Est.

$\frac{1955}{\text { Eormula, Best Est. }}$
$.09 *$
.25
.69
.64
.38
$.10 *$
$.11 *$
$.27 *$
$.06 *$
$.16 *$
.31
.58
3.64

Formula Best Egt.

Jan.

$0.02 * *$

$0.60 *$

0.20

Feb.

.07

.38

.40

Merch

.01

$.67 *$

.67

Apri1

.01

May

.34

June

.12

JuIy

.03

Aug.

.17

Sept.

.14

Oct.

.10

Nov.

.30

Dec.

$.08 * *$

TOTAL

2.04

$.18 *$

.49

.35

.28

.53

.42

.31

.40

.12

.22

.36

.40

.37

.37

.19

.39

.39

.38

4.45

4.61

1258

Formula Best Egt.. Formula

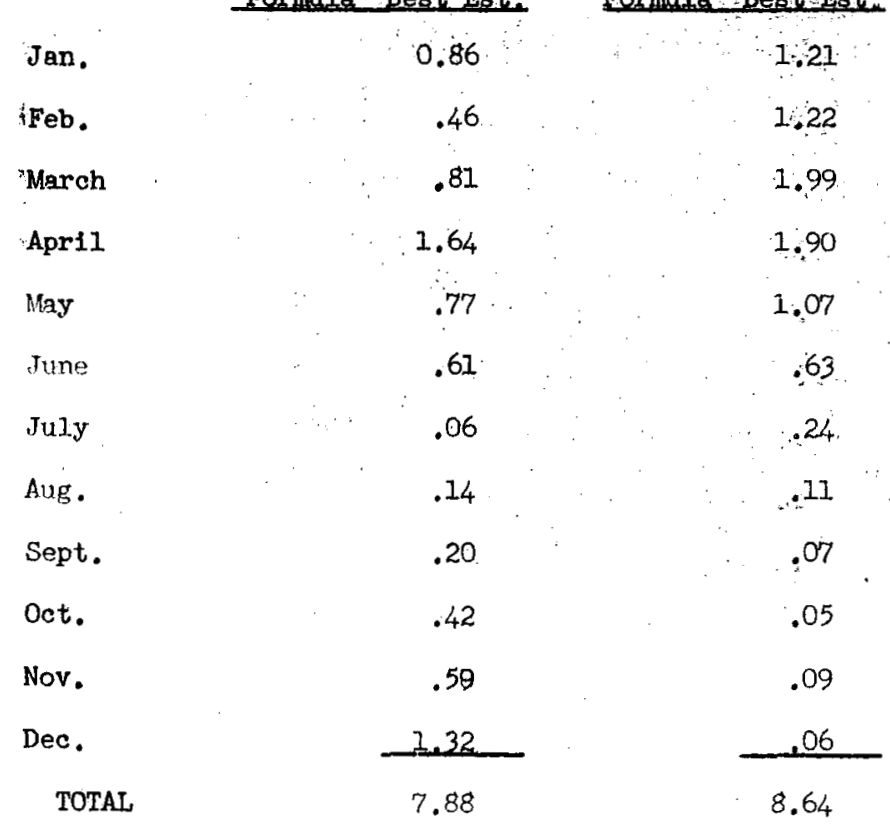

\begin{tabular}{rl} 
Pre-1954 & 0.84 \\
1954 & 2.04 \\
1955 & 3.64 \\
1956 & 4.45 \\
1957 & 4.61 \\
1958 & 7.88 \\
1959 & 8.64 \\
\hline Total & 32.1
\end{tabular}


STATION \# 401

LOCATION SEATTLE, WASHINGTON

1954

2955 Formule Best Est.

Jen.

$0.0 * *$

$0.2 *$

.5

Feb.

.1

1.3

March

Apr11

.0

1.2

May

.1

2.2

.7

June

.5

July

.1

Aug .

.5

Sept.

oct.

.4

Nov.

.2

Dec.

TOTAL

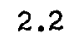

.2*

$.2 *$

$.5 *$

$.1 *$

$.3 *$

.5

1.0

6.7

$\frac{2956}{1.0 *}$
.6
$1.0 *$
$.3 *$
.5
1.0
.7
.3
.7
.7
.4
.7
7.9

Tormuda Best Est.

0.3

.7

1.1

.9

.5

.8

.7

.3

.8

.8

.9

$.2 * *$

6.5

8.5 Formula Best Est.

Jan.

Feb.

March

April

May

June

Ju.ty

Aing.

Sept.

oct.

Nov.

Dec.

TOTAL
1.4

.8

1.5

2.8

1.5

1.2

.2

.3

.5

1.4

1.7

3.2

16.5

1959

Formula Best Este

2.6

2.4

3.8

3.4

Pre-1954 2.2

1.9

1.1

.4

.2

.1

.1

$1954 \quad 6.5$

$1955 \quad 6.7$

$1956 \quad 7.9$

$1957 \quad 8.5$

$1958 \quad 16.5$

$1959-16.2$

Total

62

.1

.1

16.2 


\section{ESTIMATE OF Sr ${ }^{90}$ DEPOSITION FROM GUMMED EIIM}

STATION \# 404

LOCATION__ MEDEOED, OBECON

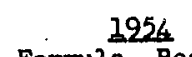

1254

Eormule Beat Est.

Formule Best Est.

Eormula Best Est.

Formulie Best Est.

Jan:

0.02

$0.06 *$

$0.39 *$

0.21

seb:

.06

.20

Maroh

.01

.32

April

.03

May

.09

June

.05

July

.05

Aug.

.03

Sept.

.05

oct.

.05

Nov.

.07

Dec.

.02

TOTAL

.53

.41

.20

.10*

$.01 *$

.01*

$.05 *$

.30

.46

$.20 *$

.55

.37*

.33

.50

.43

.27

.11

.29

.17

.16

.06

.27

.41

$.03 *$

.29

.33

17

.51

.14

39

2.07

.13

3.59

3.25

Formula Best Este Formule Best Est.

Jan.

0.35

0.80

Feb.

.32

.63

March

1.95

Apr11

May

1.54

.37

June

.68

July

.24

Aug.

.19

Sept.

.12

Oct.

.36

Nov.

Dec.

TOTAL

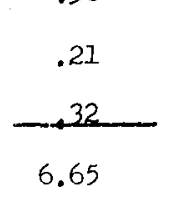

.67

.54

1.28

.36

.09

.05

.05

.07

\begin{tabular}{rr} 
Pre-1954 & 0.77 \\
1954 & .53 \\
1955 & 2.07 \\
1956 & 3.25 \\
1957 & 3.59 \\
1958 & 6.65 \\
1959 & 4.89 \\
\hline Total & 21.8
\end{tabular}

.04

.31

4.89 
ESTTMATE OF INFINITY $y$ DOSE FROM GUMMED FILM

STATION \#_ 404

WOCATION_MEDFORD, ORECON
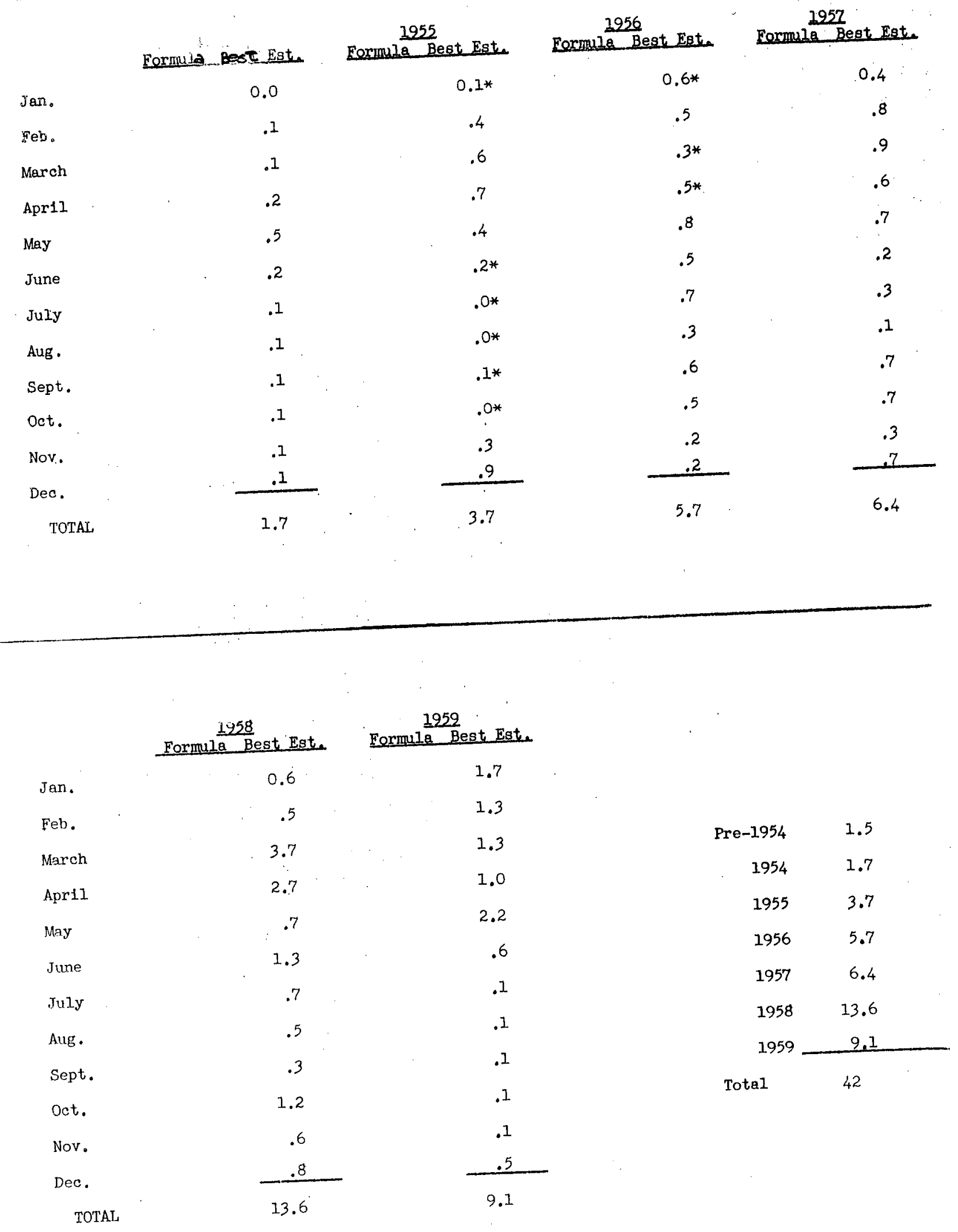


\section{ESTIMATE OF Sr ${ }^{90}$ DEPOSITION FROM GUMMED FILM}

STATION

LOCATION SAN FFANCISCO, CALIFORNIA

2954

1955

Eormula Best Est.

Jen.

Feb。

March

Apri1

May

June

Juiy

Aug.

Sept.

oct.

Nov.

Deo.

TOTAL

\begin{tabular}{l}
.01 \\
.08 \\
.03 \\
.02 \\
.11 \\
.05 \\
.02 \\
.03 \\
.06 \\
.05 \\
.05 \\
.06 \\
\hline .57
\end{tabular}

\section{Eormila Best Est.}

$0.10 *$

.16

.15

.38

$.83 \quad .32$

$.05 *$

$.04 *$

$.02 *$

$.20 *$

$2.81 *$

.20

1.01

5.44
1956 Eormula Best Est.

$0.87 *$

.42

$.23 *$

$.29 *$

.17

.10

.11

.08

.12

. .32

.06

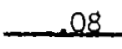

2.85
3.70

1957

Eormila Best Est.

0.49

.47

.55

.33

.38

.15

.21

.09

.40

.21

.06

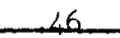

Jan.

Feb.

0.37

March

.55

3.21

April

May

June

July

Aug .

Sept.

Oct.

Nov.

Dec.

TOTAL
1.78

.34

.21

.09

.10

.07

.10

.12

.22

7.16
0.91

1.01

.61

.42

.32

.10

.04

.06

.06

.04

.04

.06

3.67

\begin{tabular}{rr} 
Pre-1954 & 0.67 \\
1954 & .57 \\
1955 & 5.44 \\
1956 & 2.85 \\
1957 & 3.70 \\
1958 & 7.16 \\
1959 & 3.67 \\
\hline & 24.1
\end{tabular}


STATION \#__ 407

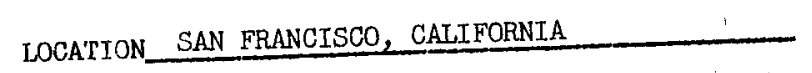

1955 Formula Best Eate

\begin{tabular}{|c|c|c|c|}
\hline Jan. & 0.0 & & $0.2^{*}$ \\
\hline $\mathrm{Feb}$ & .1 & & .3 \\
\hline March & .4 & & .3 \\
\hline Apr11 & .1 & & .7 \\
\hline May & .6 & 1.5 & .6 \\
\hline June & .2 & & $.1 *$ \\
\hline July & .1 & & $.2 *$ \\
\hline Aug. & .1 & & $.0 *$ \\
\hline Sept. & .1 & & $.3^{*}$ \\
\hline Oct. & .1 & & $4.5 *$ \\
\hline Nov. & .1 & & .3 \\
\hline Deo. & .1 & & 1.7 \\
\hline TOTAL & 2.0 & & 9.1 \\
\hline
\end{tabular}

2956 Eormule Best Est.

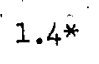

.7

$.3 *$

$.4 *$

.3

.2

.3

.2

.3

.6

.1

.2

5.0
0.8

.8

.9

.6

.6

.3

.2

.1

.7

.5

.1

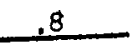

6.4

1958 Formula Best Est.

Jan.

Feb.

March

Apr 11

May

June

July

Aug.

Sept.

oct.

Nov.

Dec.

TOTAL.
0.6

.9

6.0

3.2

.7

.4

.2

.3

.2

.4

.4

$-.5$

13.8
1959

Formule Best Est.

2.0

2.0

1.2

.8

.6

.2

.1

.1

.1

.1

.1

.1

\begin{tabular}{rr} 
Pre-1954 & 1.4 \\
1954 & 2.0 \\
1955 & 9.1 \\
1956 & 5.0 \\
1957 & 6.4 \\
1958 & 13.8 \\
1959 & 7.4 \\
\hline Total & 45
\end{tabular}

7.4 


\section{ESTIMATE OF Sr 90 DEPOSITION FROM GUMMOD FILM}

STATION \# 410

LOCATION LOS ANGEIES, CAJIFORNIA

\section{Formula Best Dst.}

Jan.

Feb.

March

Apr11

May

June

July

Aug .

Sept.

oct.

Nov,

Dec.

TOTAL

\subsection{4}

.04

.01

.04

.19

.06

.04

.05

.08

.04

.19

.16

.94 $\therefore \quad 2952$ Ponmula Best Est:

$0.10 *$

.10

.13

.29

1.74

.48

$.08 *$

$.04 *$

$.04 *$

$.40 *$

$.03 *$

.49

.16

2.34
2956

Eormila Best Esta Eormula Best Est.

$0.23 *$

.30

0.45

.27

$.14 *$

$.38 *$

.37

.56

$.29 \quad .20$

$.06 \quad .16$

.17

.39

.06

.08

.09

.14

.09

.19

.09

.15

.16

.46

2.05

3.43

Formula Best Est. Formula Best Est.

Jan.

Feb.

March

Apr11

May

June

July

Aug.

Sept.

Oct.

Nov.

Dec.

TOTAL
0.23

.36

.93

.76

.27

.05

.06

.15

.08

.24

.18

.12

3.33
0.42

1.54

.42

67

$\because .16$

.08

. .06

. .06

.05

.09

.02

.11

3.68

\begin{tabular}{rr} 
Pre-1954 & 0.65 \\
1954 & .94 \\
1955 & 2.34 \\
1956 & 2.05 \\
1957 & 3.43 \\
1958 & 3.33 \\
1959 & 3.68 \\
\hline
\end{tabular}




\section{ESTTMATE OF INFINITY y DOSE FROM GUMMD FILM}

STATION \#- 410

IOCATTON IOS ANGELES, CALIFORNIA

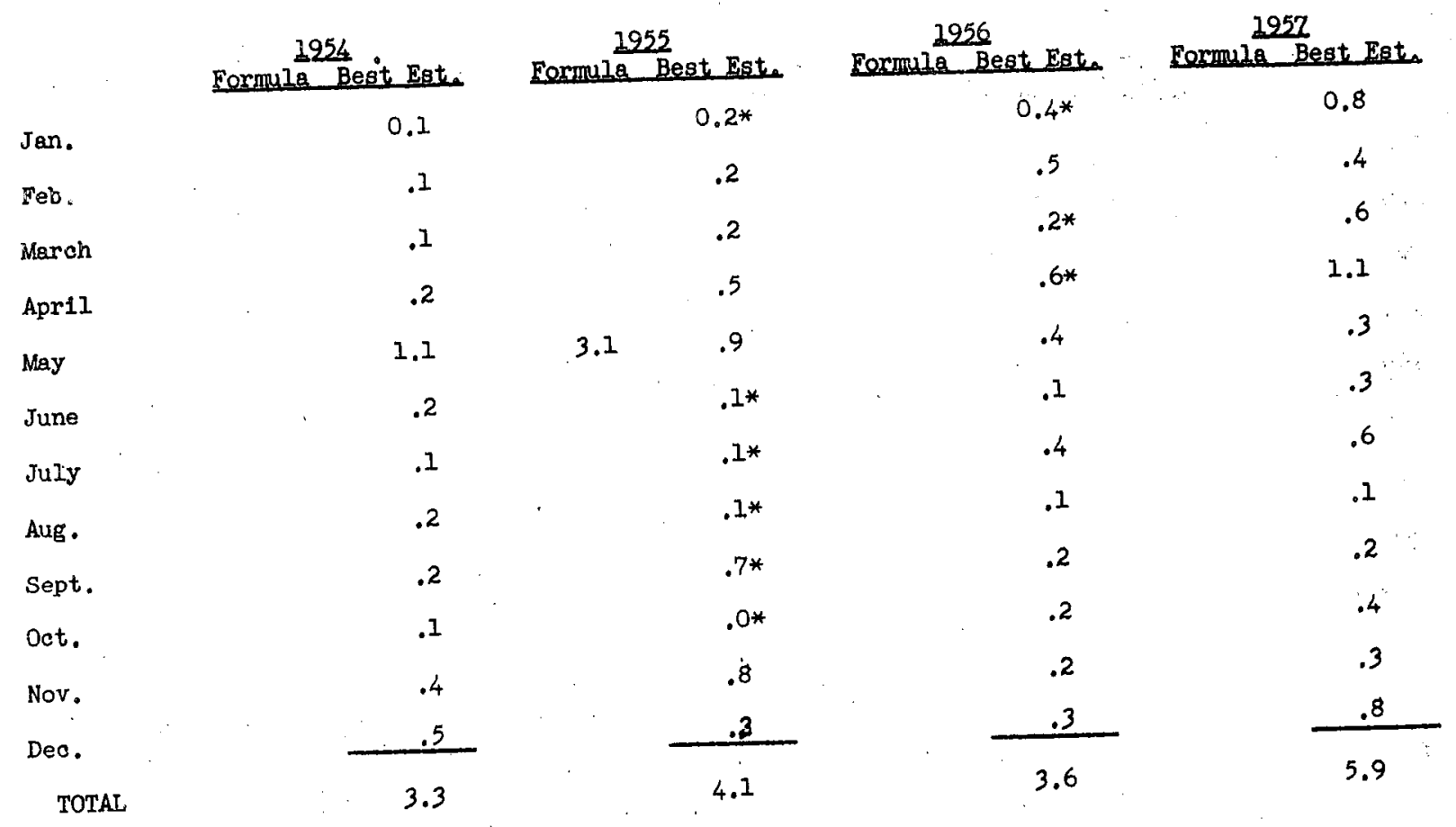

Formula Best Este Formula Best Este

$\begin{array}{lcc}\text { Jan. } & 0.4 & 0.9 \\ \text { Feb. } & .6 & 3.1 \\ \text { March } & 1.9 & .8 \\ \text { Apri1 } & 1.3 & 1.2 \\ \text { May . } & .5 & .3 \\ \text { June } & .1 & .1 \\ \text { July } & .2 & .1 \\ \text { Alug. } & .4 & .1 \\ \text { Sept. } & .2 & .1 \\ \text { Oct. } & .6 & .1 \\ \text { Nov. } & .5 & .0 \\ \text { Dec. } & .3 & 7.0 \\ \text { Total. } & 7.0 & .2\end{array}$

$$
\begin{array}{rr}
\text { Pre-1954 } & 1.2 \\
1954 & 3.3 \\
1955 & 4.1 \\
1956 & 3.6 \\
1957 & 5.9 \\
1958 & 7.0 \\
1959 & 7.0 \\
\hline \text { Total } & 32
\end{array}
$$


STATION \# 501

WOCATION ANCHORAGE, AIASKA

$\therefore \quad 1254$

Formule Best Est.

1955

Formula Best Est.

Formuia Best Est.

1957

Jan.

0.04

$0.00 *$

$0.25 *$

Formula Best Est.

Feb.

.03

.15

March

.00

.13

ApriI

.01

.26

May

.06

.06

June

.03

$.17 *$

JuIy

.06

Aug .

.05

Sept.

.10

Oct.

.09

Nov.

.05

Dec.

TOTAL

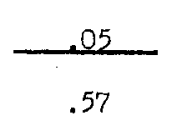

$.15 *$

$15 \quad .41$

0.16

.22

$.21 *$

.16

$.35 *$

.10

.30

.17

.30

.21

.07

.26

$.17 *$

.19

.22

$.07 *$

.31

.69

$.34 *$

.12

.18

.31

.11

.06
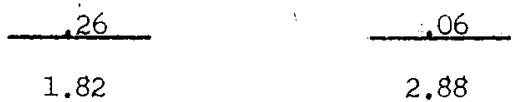

.13

2.61

Formila Best Este. Formula Best Est.

Jan.

0.30

0.32

Feb.

.17

.38

March

.16

Apr11

.67

May

.42

Tune

.45

July

.20

.34

.44

.60

.37

.44

$\begin{array}{rr}\text { Pre-1954 } & 0.74 \\ 1954 & .57 \\ 1955 & 1.82 \\ 1956 & 2.88 \\ 1957 & 2.61 \\ 1958 & 3.57 \\ 1959 & \end{array}$

Aug.

.16

Sept.

.15

Oct.

.54

Total

Nov.

Dec.

TOTAL,

.18

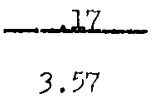


$1954 \quad 1955$ Formula Best Eeta

Jan.

Feb.

March

Apri1

Mry

June

JuLy

Aug .

Sept.

oot.

Nov.

Deo.

TOTAL
0.0

.1

.0

.1

.3

.1

.2

.1

.3

.2

.1

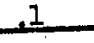

1.6
Formize Best Est.

$0.0 *$

.3

.2

.5

.1

$.3 *$

$.3 *$

$.3 *$

.1*

$.5 *$

.1

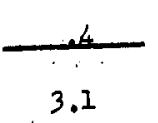

1956 Formula Best Est $\therefore$ Formula Begt Bat.

$$
0.4^{*}
$$

.7

$.3 *$

$.5 *$

.5

.6

.2

.4

.7

.2

.7

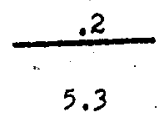

0.3

.4

.3

.2

.3

.4

.4

.3

1.2

.4

.2

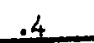

4.6

1958 Formula Best Est.

0.5

Jan.

.3

Feb.

March

.3

Apri1

1.1

May

June

July

Aug.

Sept.

oct.

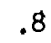

.9

.5

.4

.4

Nov.

1.5

Dec.

TOTAL,

1959

Eormula Best Eat.

0.7

.8

.6

.8

1.0

.6

.7

$\begin{array}{rr}\text { Pre-1954 } & 2.0 \\ 1954 & 1.6 \\ 1955 & 3.1 \\ 1956 & 5.3 \\ 1957 & 4.6 \\ 1958 & 7.6\end{array}$

1959

Total 


\section{ESTIMATE OF SY 90 . DEPOSITION FROM GUMMED FILM}

STAITON \#_ 502

IOCATION NORTH BAY, ONTARIO, CANADA

1254

1957

Formila Best Est. Formula Best Est. Formila Best Este Formila Best Est.

Jan.

0.04

$0.05 *$

$0.13 *$

0.20

Feb.

.09

.66

.10

.29

March

.01

2.59

.75

2.14

1.15

$.33 *$

.14

Apr11

.04

1.18

May

.03

$.20 *$

June
JuIy

.11

$.09 *$

Aug .

.08

$.09 *$

Sept.

.09

$.3 .7 *$

Oct.

.33

$.31 *$

Nov.

.147

.23

$.30 *$

.59

Dec.

.26

TOTAL

.01

1.23

5.35

.77

.81

.35

$.50 * *$

.19

$.65 * *$

.20

.38

.48

.99

.37

.31

.22

.32

.27

5.55

1958

Formuli Best Est.

1959

0.29

Formula Best Est.

Jan.

.36

0.41

Feb.

.45

.38

March

1.64

.76

April

2.15

1.34

May

.92

1.67

June

.19

July

.32

Alug.

.24

Sept

.48

Oct.

.46

Dec.

$-477$

6.97

.75

.34

.13

.14

.10

3.57

20
.29
.4
.59
.1
$.0 * *$
$65 * *$
38
.99
.37
31
32
.55


STATION \# 502

LOCATION NORTH BAY, ONTARIO, CANADA.

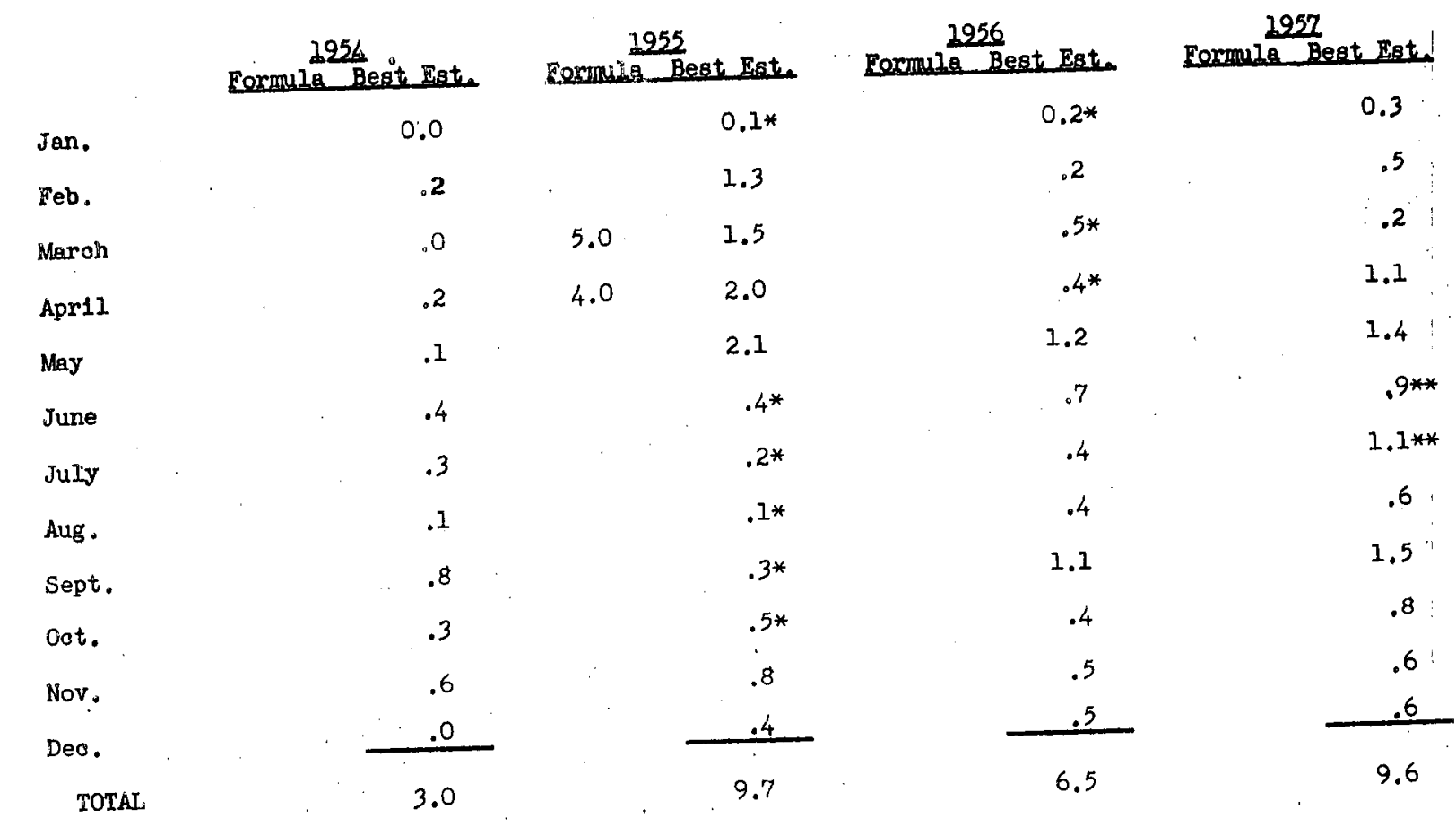

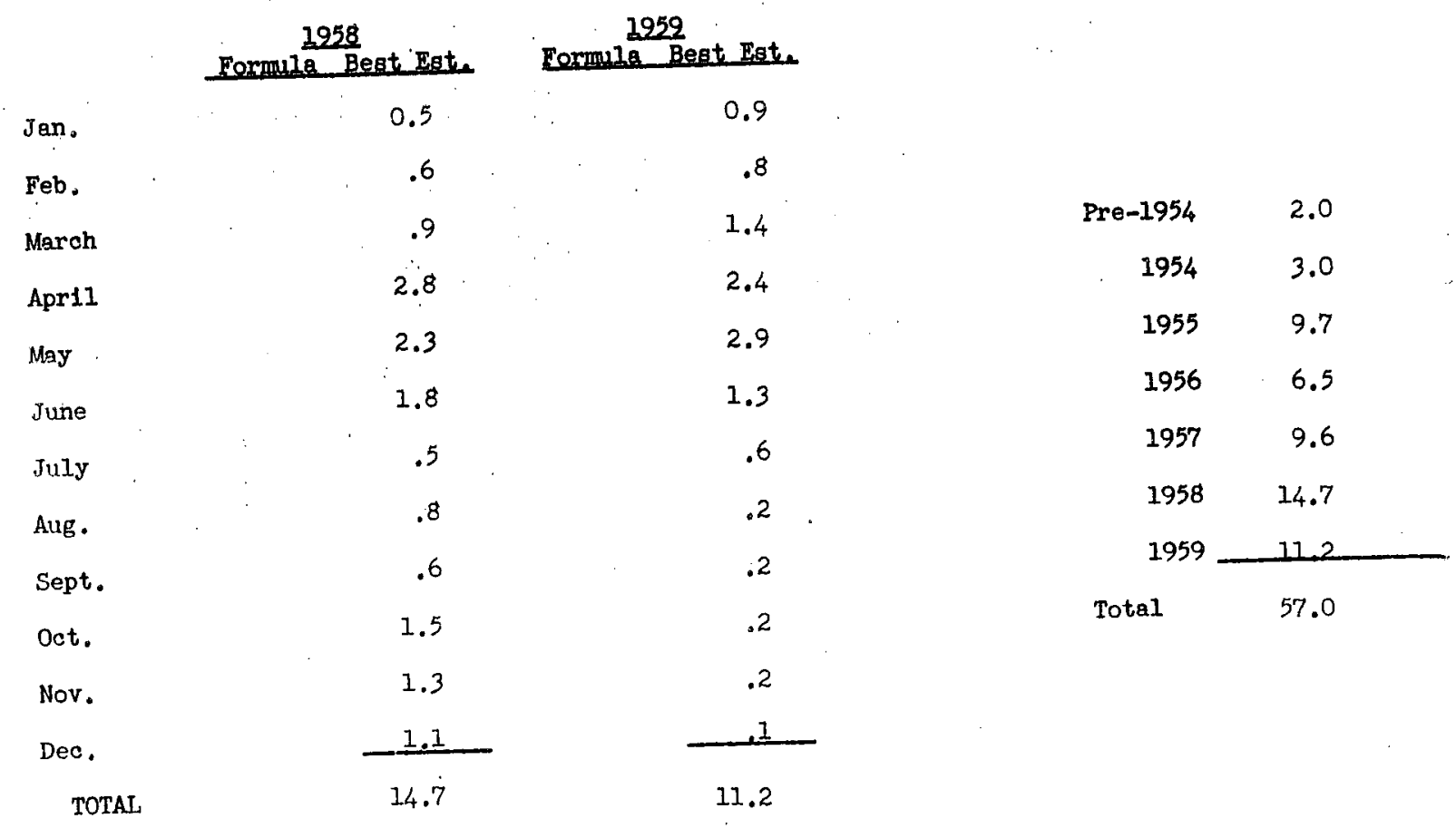




\section{ESTYMIATE OF Sr90 DEPOSITION FROA GUMUDO ETLM}

STATTON \# 503

LOCATION WOWWUEE, ONTAFIS, OANADA

Formula $\frac{1254}{\text { Best Est }}$

Jan.

Feb.

March

Apri1

May

June

JuIy

Aug .

Sept.

Oct.

Nov.

Deò.

TOTAL

$$
0.04
$$

.08

.00

.01

.01

.06

.06

.09

.03

.10

.14

.01

.59
1955

Formule Besc Est

0.0 .2 .5

.11

.09

.16

.51

$.16 *$

$.23 *$

$.13 *$

$.07 *$

$.09 *$

.02

.10

1.68
1956 Forrela Begt Zsto Formula Best Est.

$0.20 *$

$.28 * *$

0.03

.23*

$.37 *$

.53

.64

.32

.15

.40

.35

.06

.06

3.59
.09

.15

.36

.73

.68

.60

.53

.90

.26

.39

.24

4.96

1958

1959

Formila Best Egta Formula Best Este

$\begin{array}{lcc}\text { Jan. } & 0.08 & 0.07 \\ \text { Feb. } & .06 & .11 \\ \text { March } & .24 & .15 \\ \text { Apr11 } & .58 & .72 \\ \text { May } & .69 & .2 .11 \\ \text { June } & .85 & 1.54 \\ \text { July } & .15 & .58 \\ \text { Aug. } & .24 & .20 \\ \text { Sept. } & .23 & .08 \\ \text { Oct. } & .21 & .10 \\ \text { Nov. } & .33 & .08 \\ \text { Dec. } & .08 & .11 \\ \text { TolaL } & 3.74 & 5.85\end{array}$

$$
\begin{array}{rr}
\text { Pre-1954 } & 1.02 \\
1954 & .59 \\
1955 & 1.68 \\
1956 & 3.59 \\
1957 & 4.96 \\
1958 & 3.74 \\
1959 & 5.85 \\
\hline & \\
\text { Total } & 21.4
\end{array}
$$


STATION \#_ 503

IOCATION MOOSOONEE, ONTARIO; CANADA

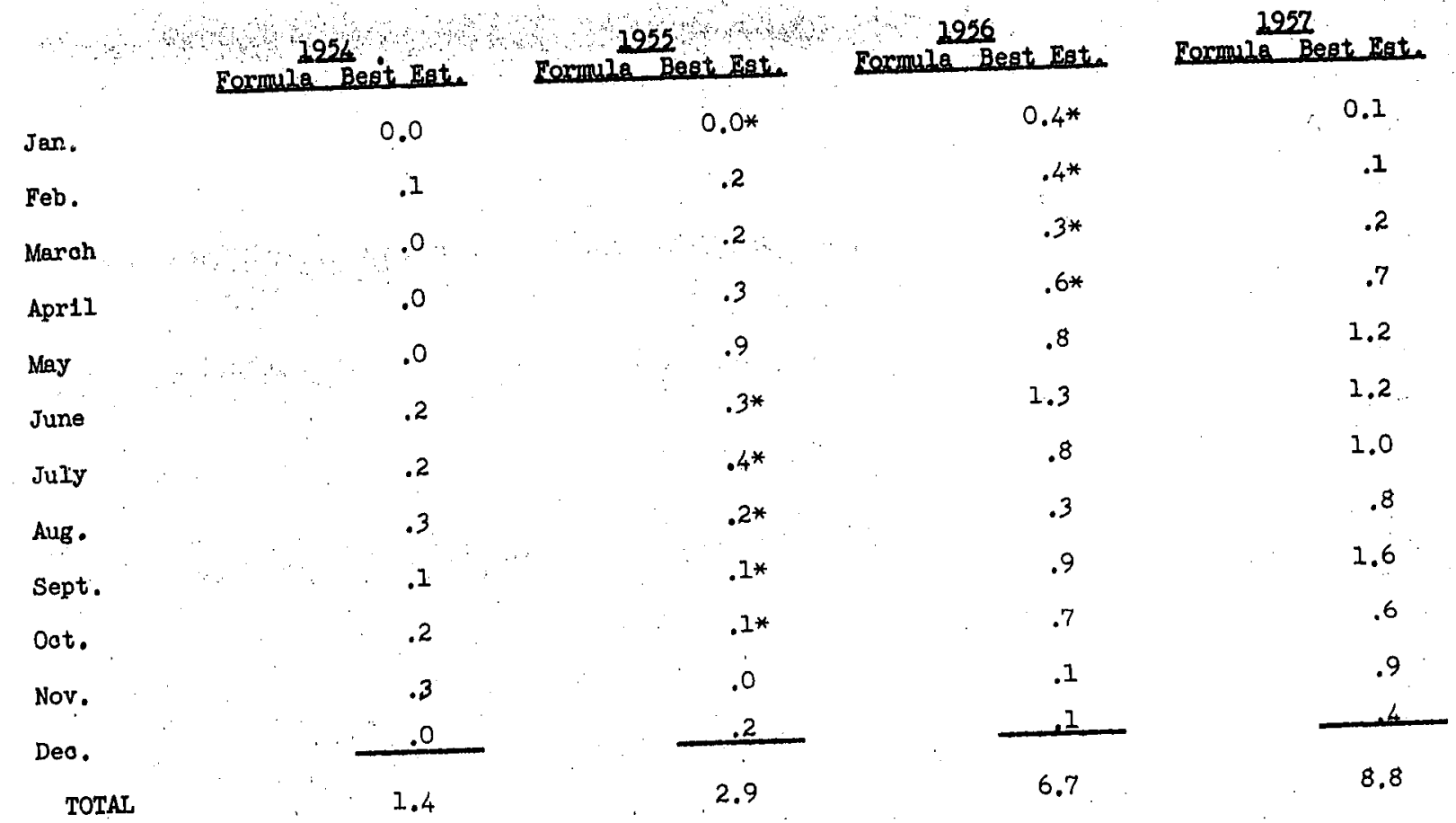

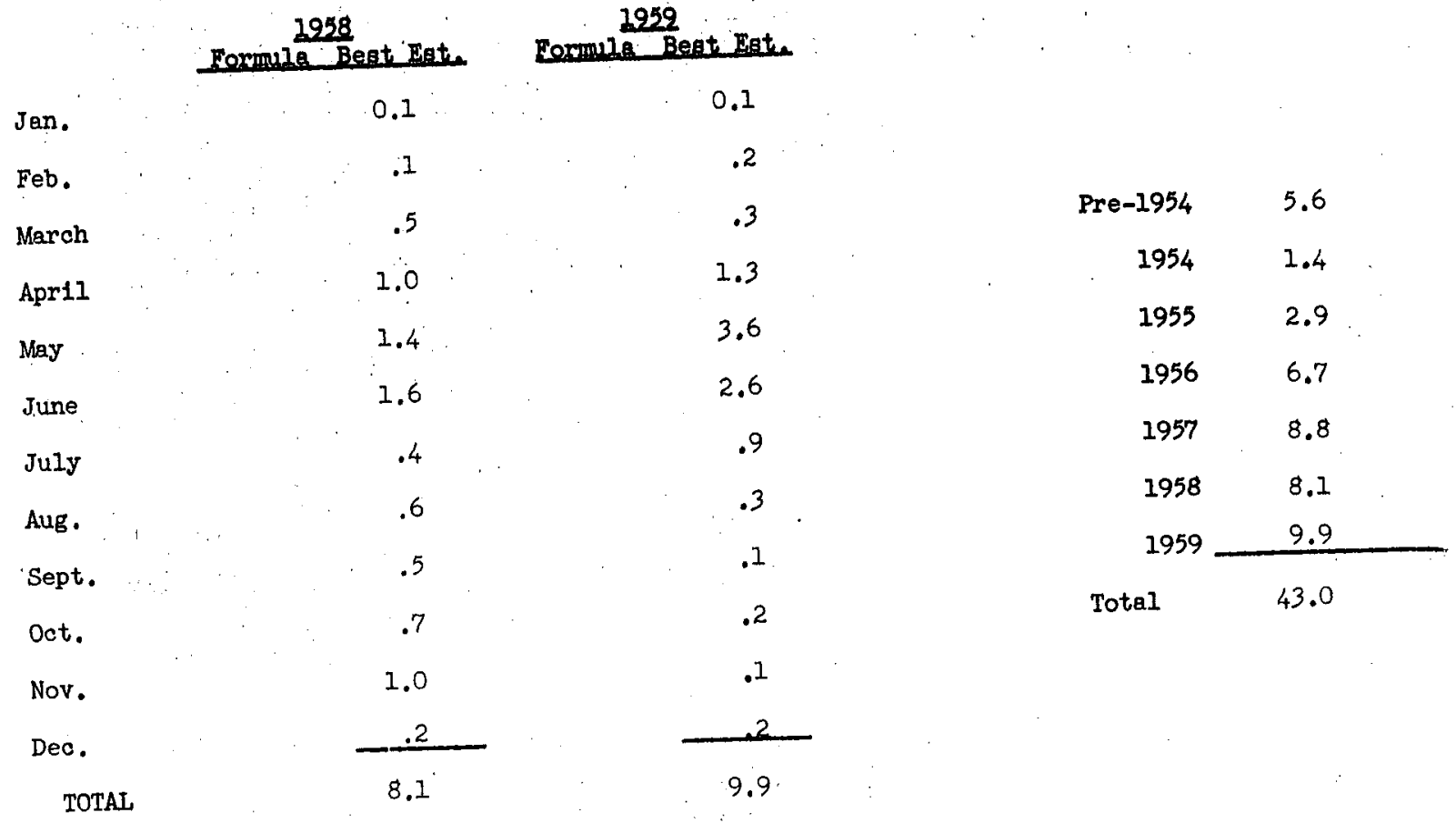


1254

Formula Best Est: Eormula Best Est.

Jan.

530.

March

Apriz

May

June

July

Aug.

Sept.

Oct.

Nov.

Dec.

TOTAL
0.03

.03

.01

.01

.01

.03

.07

.16

.12

.11

.26

.02

.86
$0.03 *$

.10

.44

.34

.71

$.15 *$

$.14 *$

$.12 *$

$.07 *$

$.12 *$

.07

.28

2.47
1956

Formula Best Est.

$0.61 *$

.06

$.18 *$

$.29 *$

.57

.25

.23

.12

.33

.16

.10

.21

3.12
1957

Formule Best Est.

0.10

.08

.18

.38

.51

.38

.36

.34

.64

.28

.16

.40

3.81

1958

1959

Formula Best Este Formula Best Est.

Jan.

0.07

0.27

Feb.

.17

.30

March

.15

Apr11

.28

May

.20

June

.20

July

.08

Aug.

.10

Sept.

.19

oct.

.71

Nov.

Dec.

.38

.70

.96

.97

71

.29

TOTAI

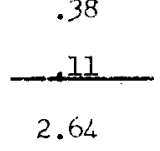

i. .09

8. .12

.11

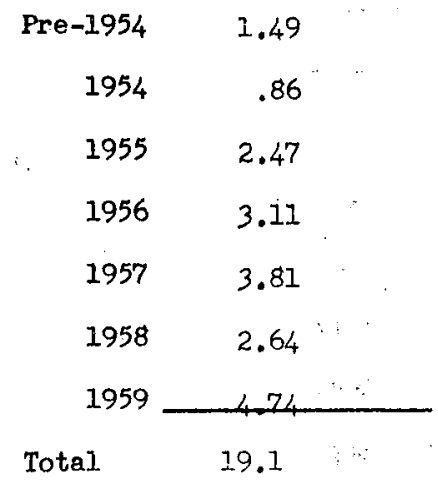

.15

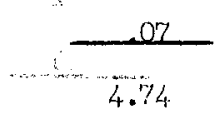




\section{ESTIMATE OF INFINITY $y$ DOSE FROM GUMMED FILM}

STATION \# 504

LOGATION MONCTON, NEW BRUNSWICK, CANADA

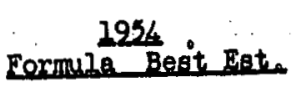

Jan.

Fob.

Maroh

Apr11

May

June

JuIy

Aug.

Sept.

oot.

Nov.

Deo.

TORAL

$$
0.0
$$

.0

.0

.0

.1

.1

.2

.5

.6

.3

.6

1

2.5

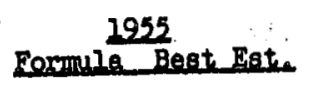

$0.1 *$

.2

.8

.6

1.3

$.3 *$

.2*

$.2 *$

.1*

$.2 *$

.1

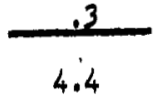

2956

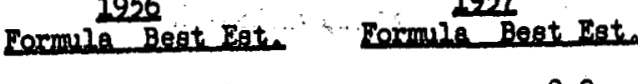

1.0*

.1

.3*

$.4 *$

.9

.5

.5

.3

.7

.3

.2

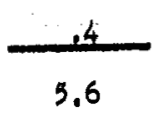

0.2

.1

.3

.7

.8

.7

.6

.5

1.0 :

.6

.3

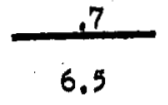

2958

Dommita Bent inat.

Jan.

0.1

Feb.

$: 3$

Maroh

Apr11

May

June

July

Aug.

Sept.

.3

.5

.4

.4

.2

.3

.4

Oct.

2.5

Nov.

Dec.

TOTAL

1059

Dovmil Bant mat.

0.6

.6

1.3

1.7

1.7

1.2

.5

.1

.2

.2

Pre-1954 $\quad 3.6$

29542.5

$1955 \quad 4.4$

$1956 \quad 5.6$

$1957 \quad 6.5$

$1958 \quad 6.8$

1959

8

Total

38.0 
1954

1256

1957

Eormula Best Est. Formule Best Est Eormule Best Este

Formula Best Est.

Jạn.

0.03

$0.02 *$

$0.22 *$

0.13

Feb.

.06

.17

.07

.18

March

$1.38 \quad .94$

Apr1I

.01

$1.44 \quad .88$

$.26 *$

.23

May

.93

$.53 *$

.46

June

.02

1.00

.76

JuIy

$.26 *$

Aug.

$.10 *$

Sept.

$.10 *$

$.14 *$

oct.

$.08 *$

Nov.

.15

.22

.41

.08

.21

.54

.06

.14

.24

.35

1.06

.17

.18

.27

Dec.

.31

.26

.13

.19

TOTAL

.28

1.13

4.03

.26

.42

3.57

4.89

Formula Best Est.

1959

0.29

Formi1a Best Est.

Jan.

.60

1.27

Feb.

.36

.94

March

.69

Apri1

1.40

1.46

May

1.17

.59

June

.87

.88

July

.31

.41

Aug.

.21

.28

Sept.

.32

.16

Det.

.39

.24

\begin{tabular}{rr} 
Pre-1954 & 1.00 \\
1954 & 1.13 \\
1955 & 4.03 \\
1956 & 3.57 \\
1957 & 4.89 \\
1958 & 6.79 \\
1959 & 7.26 \\
\hline Total & 28.7
\end{tabular}

Nov.

.45

.27

Dec.

$-.42$

TOTAL

6.79

.07

7.26 
ESTIMATE OF INFINITY $\gamma$ DOSE FROM GUMMED FILM

STATION \# 505

LOCATION MONTREAL, QUEBEC, CANADA

1254

2955

1956 .

Coxula Best Est.

Formia Best Est.

Formula Best Eat.

Eormula Best Est.

$0.0 *$

Jan.

0.0

.3

$0.3 *$

0.2

Feb.

.1

2.7

1.8

Merch

.$I$

2.7

1.6

Apr11

.1

May

.1

June

.3

JuIy

.3

Aug .

.2

sept.

oct.

.3

Nov.

.4

Deo.

.7

TOTAL

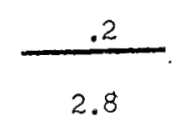

1.7

$.4 *$

$.2 *$

. $2 *$

$.2 *$

.1*

.2

.4

7.1

.1

$.4 *$

$.8 *$

1.5

.4

.4

.3

.4

.8

1.3

.8

.9

.4

1.6

.5

.4

.2

.5

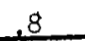

6.1

8.4

Jan.

Feb.

March

Apri1

May

June

July

Aug.

Sept.

oct.

Nov.

Dec.

TOTAL
0.5

1.0

.7

2.4

2.3

1.7

.8

.5

.8

1.5

1.3

$-1.0$

14.5
2.7

1.9

1.3

2.6

1.0

1.5

.7

.4

.3

.4

\begin{tabular}{rr} 
Pre-1954 & 3.2 \\
1954 & 2.8 \\
1955 & 7.1 \\
1956 & 6.1 \\
1957 & 8.4 \\
1958 & 1.4 .5 \\
1959 & 13.3 \\
\hline Totel & 55.0
\end{tabular}

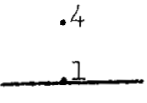

13.3 
ESTIMATE OF Sr 90 DEPOSITION FROM GUMMED FIIM

STATION \#_ 507

IOCATION SEVEN ISLANDS, QUEBEC, CANADA

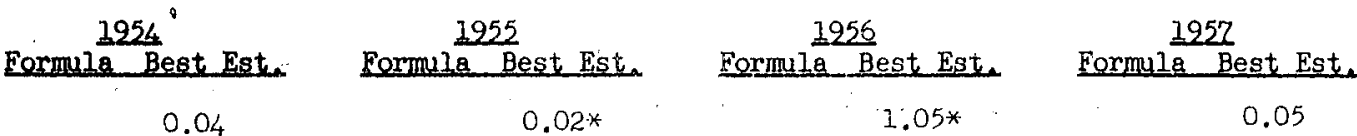

Jan.

0.04

.15

Feb.

.05

.23

Merch

.00

April

May

.02

.09

June

.02

.30

July

.03

Aug.

.08

Sept.

.09

oct.

.08

Nov.

.22

Deo.

.32

TOTAL

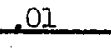

.96

$.25 \%$

$.27 *$

$.17 *$

$.12 *$

$.03 *$

.05

$.11 *$

.10

.12

.26

.45

.58

.33

.58

.19

.46

.19

.38

$.11 *$

.27

1.39

.20

.30

.30

$.10 * * *$

.08

.49

$\frac{.27}{3.32}$

.18

2.48

4.41

1958

Formula Best Est.

Jan.

0.09

Formul Best Est.

0.09

Feb.

.12

.10

March

.13

.08

Apr11

.72

.40

May

.67

June

.90

July

.17

Aug.

.26

Sept.

.23

oct.

.30

Nov.

.26

Dec.

TO'TAL

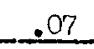

3.92

1. 32

1.21

.54

.32

.47

.21

\begin{tabular}{rr} 
Pre-1954 & 1.12 \\
1954 & .96 \\
1955 & 2.48 \\
1956 & 3.31 \\
1957 & 4.41 \\
1958 & 3.92 \\
1959 & 4.96 \\
\hline Total & 21.2
\end{tabular}

.14

$\frac{.08}{4.96}$ 


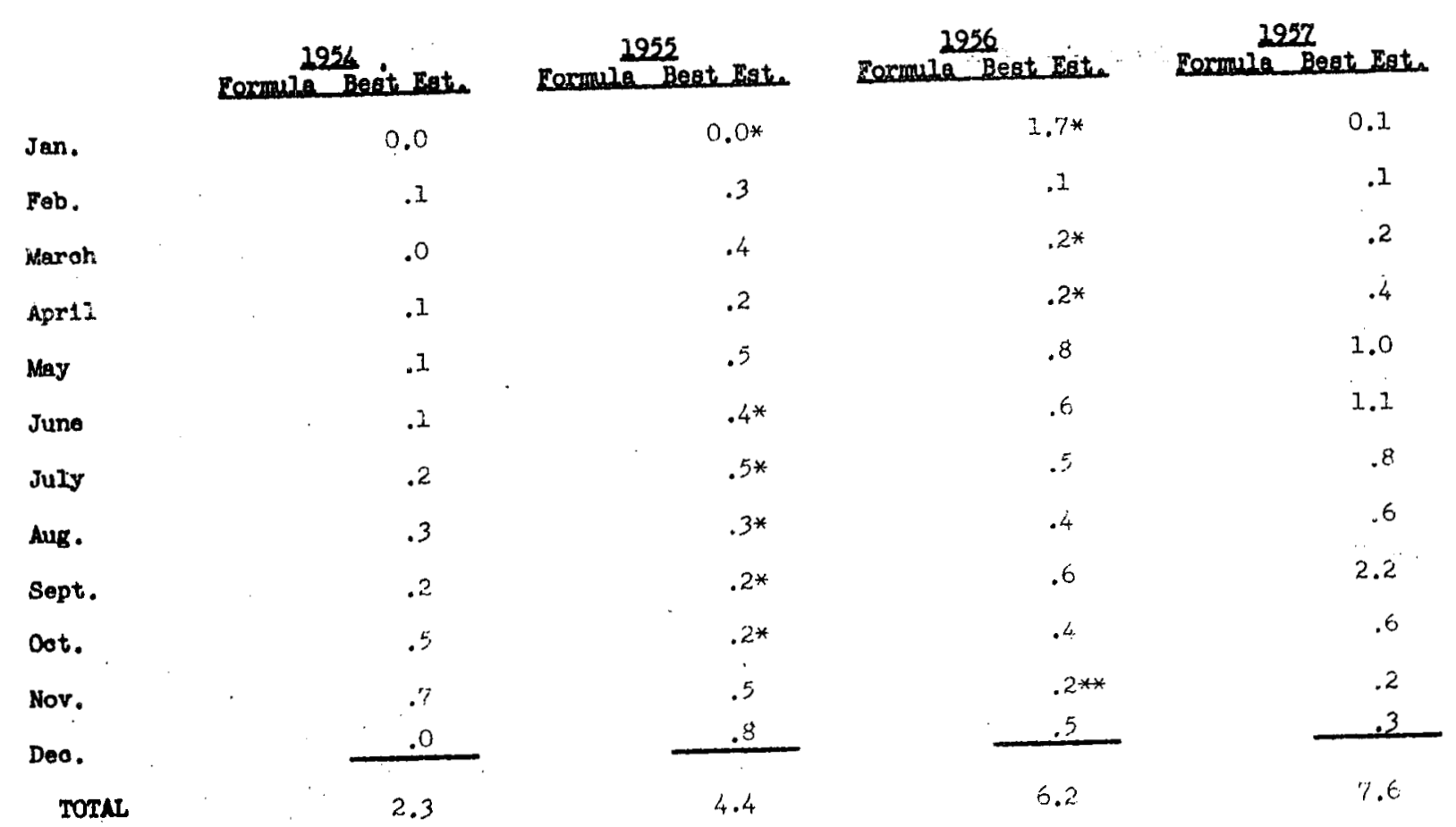

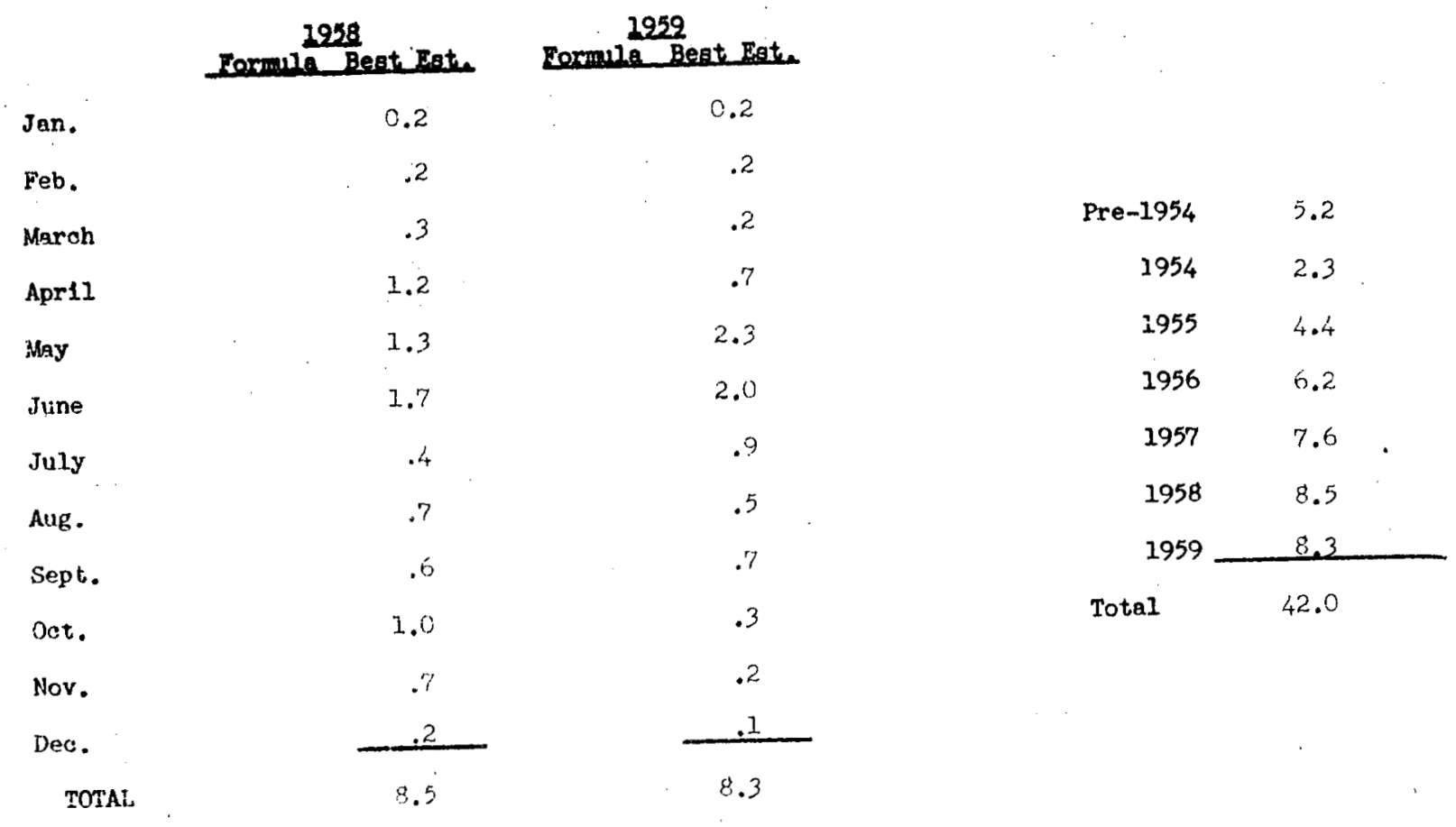


ESTTMATE OF Sr90 DEPOSITION EROM GUMMO ELUM.

STATTON \# 508

LOCATION WINNIPEG, MANITOBA, CANADA

Foxmula Beat jet.

0.04

Jan.

Feb.

Merch

A.priI

May

June

JuIy

Aug.

Sept.

Dot.

Nov.

Dec.

TOTAL
.03

.02

.02

.05

.08

.08

.04

.09

.07

.07

$\frac{.08}{.67}$
1255

Formuia Best Est.

$0.03 *$

.05

.11

.80

$1.67 \quad 1.01$

$.34^{*}$

$.16 *$

$.13 *$

$.20 *$

$.23 *$

.13

.46

3.55
1956

Eormulie Best Est.

$0.83 *$

$.05 *$

$.16 * *$

$.11 *$

.91

.28

.24

.25

.39

.24

.13

.14

3.73
2957

Cormila Best Est.

0.19

1.74

.56

.46

.45

.89

4.38

1.58

1.00

.83

.34

.05

.34

8.43

1958

Formule Best Eat. Eormule Best Est.

Jan.

0.08

i. 05

Feb.

.30

March

.78

$.15 * *$

Apr11

1.06

.91

May

1.12

June

.46

Juiy

Aug.

Sept.

oct.

Nov.

Dec.

TOTAL

.17

.34

.24

.22

.34

1.15

6.25

1.25

1.14

.70

.49

.14

.13

.08

.06

6.38

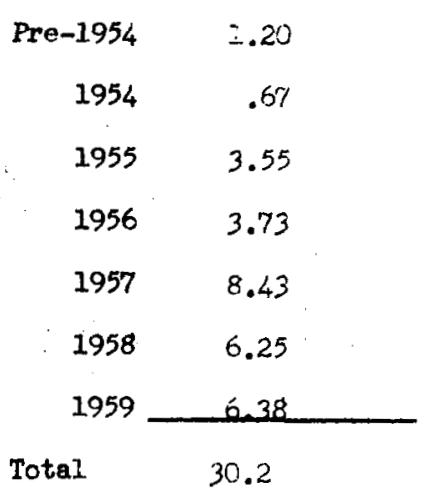




\section{ESTIMATE OF INFINITY $Y$ DOSE FROM GUMMED FILM}

STATION \#

LOCATION WINNIPEG, MANITOBA, CANADA

1954 Eormula Best Eat.

Jan,

Feb.

Maroh

Apr11

May

June

July

Aug.

Sept.

oot.

Nov.

Deo.

TOTAL
0.0

.0

.1

.1

.3

.3

.3

.1

.1

.1

.1

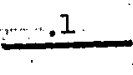

1.6
1955 Formile Begit Est.

$0.1 *$

.1

.2

1.5

3.0

1.9

$.6 *$

$.3 *$

$.2 *$

$.2 *$

$.4^{*}$

.2

. .8

6.5
1956 Tormula Begt Eat.

$1.3 *$

$.1 *$

$.3 * *$

$.2 *$

1.4

.5

.5

.5

.9

.5

.$^{2}$

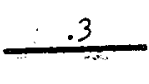

6.7
1957

Eormula Best Dat.

0.3

2.9

.9

.9

.7

1.6

7.1

2.6

1.6

1.3

.7

.1

.6

14.2

$\begin{array}{lcc}\text { Jan. } & 0.1 & 2.3 \\ \text { Feb. } & .5 & .3 * * \\ \text { March } & 1.5 & .5 * * \\ \text { Apr11 } & 1.8 & 1.6 \\ \text { May } & 2.1 & 2.2 \\ \text { June } & 1.0 & 1.9 \\ \text { July } & .5 & 1.1 \\ \text { Aug. } & .9 & .8 \\ \text { Sept. } & .6 & .2 \\ \text { Oct. } & .8 & .2 \\ \text { Nov. } & 1.0 & .1 \\ \text { Dec. } & 2.8 & 11.3 \\ \text { Total } & 13.6 & .1\end{array}$

Pre-1954

6.0

$1954 \quad 1.6$

$1955 \quad 6.5$

$1956 \quad 6.7$

$1957 \quad 14.2$

$1958 \quad 13.6$

$1959 \quad 11.3$

Total

60 


\section{ESTLMATE OF Sx.90 DEPOSILION FROM GUNAED FILM}

STATION \# 509

LOCATION CHURCHILI, MANITOBA, CANADA

1954

Eoxmula Best Est.

$$
1255
$$

Eormule Best Est.

2956.

Eormula Best Esti:

1957

Jen.

0.04

$0.01 *$

$0.05 *$

0.09

Feb.

.03

.02

Maroh

.01

.08

$.05 * *$

$.04 *$

.07

April

.00

.17

May

.01

.12

$.05 *$

.15

June

.04

July

.03

Aug .

.04

Sept.

.09

Oct.

.04

Nov.

Deo.

.09

TOTAL

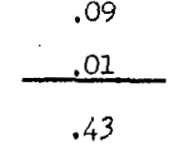

.43

$.24 *$

$.12 *$

$.11 *$

$.08 *$

.11

.11

.12

.14

.16

.10

.49

.03

.33

.21

.83

$.08 *$

.20

.13

.12

.03

.02

.07

.11

.06

1.21

1.12

2.56

(1) 1958

Formule Best Est.

1959 .

0.49

Formla Best Est.

Jan.

.22

0.07

Feb.

$: 12$

.04

March

.63

Apr 11

.10

.08

.11

May

.22

.24

June

.12

.25

July

.10

Aug.

.13

Sept.

.09

oct.

.23

Nov.

Dec.

.06

TOTAL :

$2.5 I$

.77

.36

.23

.08

\begin{tabular}{rr} 
Pre-1954 & 0.65 \\
1954 & .43 \\
1955 & 1.21 \\
1956 & 1.12 \\
1957 & 2.56 \\
1958 & 2.51 \\
1959 & 2.48 \\
\hline Total & 11.0
\end{tabular}

.04

.21

2.48 
ESTIMATE OF INFINITY y DOSE FHOM GUMMED FILM

STATION \# 509

IOCATION CHURCHIL, MANITOBA, CANADA

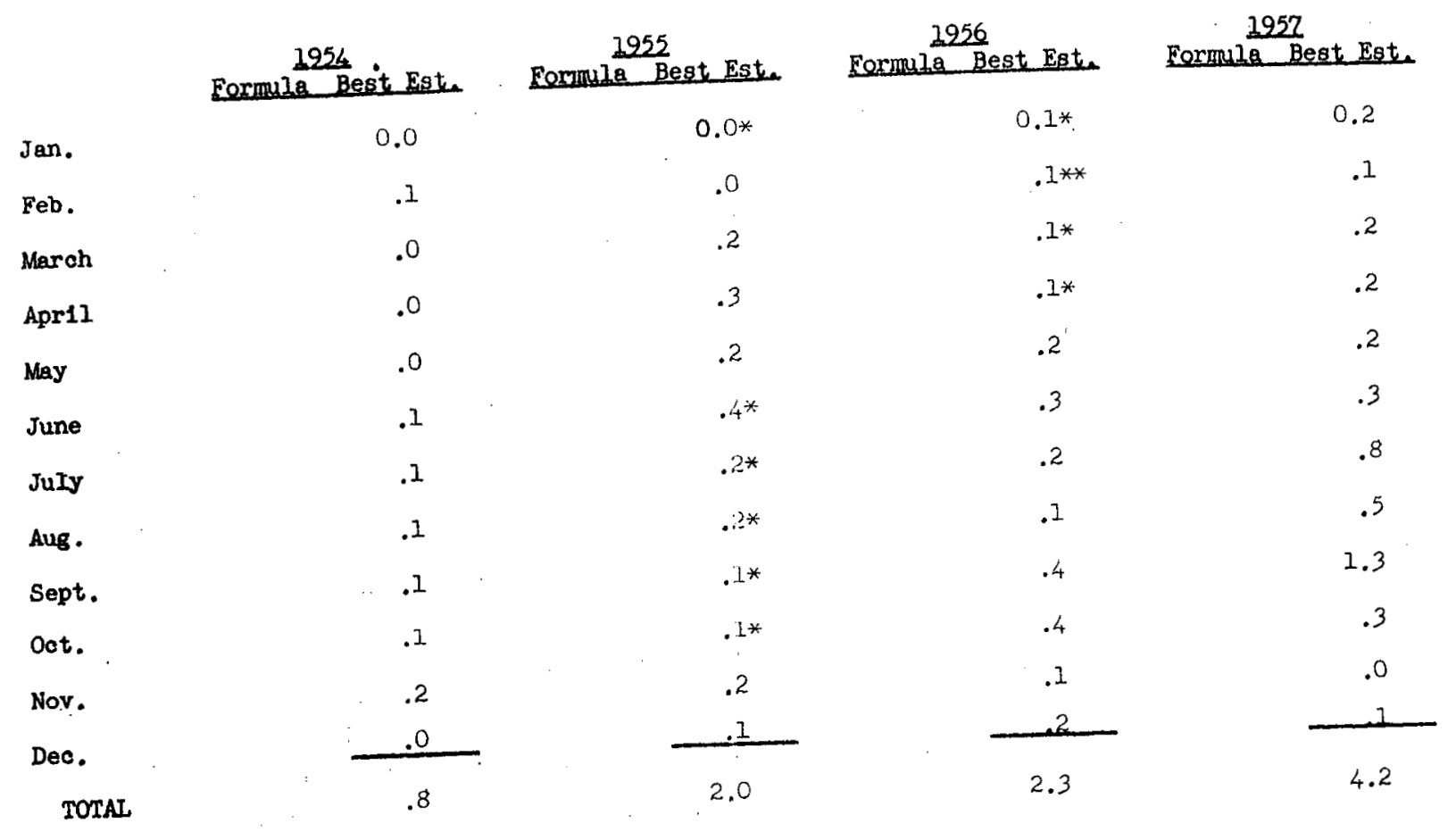

1958 Formule Best Est.

0.8

Jan.

Feb.

March

Apr11

May

June

iTuly

Aug.

Sept.

oct.

Nov.

Dec.

TORAL
.4

.2

1.1

.2

.4

.3

.3

.3

.3

.7

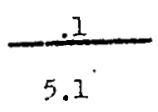

2952

Eormula Begt Est.

0.1

.1

$$
.1
$$

.2

.4

.4

1.3

.6

.4

.1

\begin{tabular}{rr} 
Pre-1954 & 1.3 \\
1954 & .8 \\
1955 & 2.0 \\
1956 & 2.3 \\
1957 & 4.2 \\
1958 & 5.1 \\
1959 & 4.1 \\
\hline & 20.0
\end{tabular}

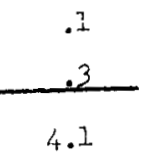




\section{ESTIMATE OF Sr 90 DEPOSITION EROM GUMMED FIIM}

STATION \# 510

LOCATION REGINA, SASKATCHEWAN, CANADA

\begin{tabular}{|c|c|c|c|c|c|}
\hline Eormula Best Este & Formul & $\frac{55}{\text { Best ist }}$ & Eormila Best Est & \multicolumn{2}{|c|}{ Formula Best Est. } \\
\hline 0.04 & & $0.01 *$ & $0.06 *$ & & 0.09 \\
\hline .02 & & .014 & .03 & & .09 \\
\hline .00 & & .05 & $.20 *$ & & .16 \\
\hline .02 & & 1.01 & $.14 *$ & & .59 \\
\hline .09 & 1.44 & .81 & .63 & & .61 \\
\hline .04 & & $.33 *$ & .80 & & .65 \\
\hline .12 & & $.14^{*}$ & .22 & 7.38 & 2.92 \\
\hline .18 & & $.15 *$ & .23 & & 2.76 \\
\hline .01 & & $.09 *$ & .24 & & 1.51 \\
\hline .16 & & $.12 *$ & .21 & & .66 \\
\hline .18 & & .15 & .06 & & II. \\
\hline .01 & & .09 & .24 & & .33 \\
\hline .87 & & 2.99 & 3.06 & & 10.48 \\
\hline
\end{tabular}

Jan.

Feb.

March

April

May

June

Juty

Aug .

Sept.

Oat.

Nov.

Deo.

TOTAL

.87

1958

Pormila Best Est.

Jan.

0.03

Feb.

Maroh

Apr11

May

June

July

Aug.

Sept.

Oct.

Nov.

Dec.

TOTAL
.17

.10

1.07

1.15

.61

.20

.45

.20

.49

.12

.10

4.69

1959

Formula Best Est.

$\therefore 0.17$

.15

.32

.92

$\therefore 1.95$

$=1.53$

$\therefore .55$

.32

.30

.14

.08

.08

6.51

\begin{tabular}{rr} 
Pre-1954 & 0.90 \\
1954 & .87 \\
1955 & 2.99 \\
1956 & 3.06 \\
1957 & 10.48 \\
1958 & 4.69 \\
1959 & 6.51 \\
\hline
\end{tabular}

Total

29.5 
STATION \# 510 Formula Best Est.

Jan.

0.0

Feb.

March

April

May

June

JuIy

Aug.

Sept.

oct.

Nov.

Deo.

TOTAL
.0

.0

.1

.5

.1

.4

.5

.0

.4

.4

.0

2.4

IOCATION REGINA, SASKATCHEWAN, CANADA

1956 Eormula Best Est.

$0.1 *$

$0.0 *$

.1

.1

1.9

2.6

1.7

$.6 *$

$.2 *$

$.3 *$

. $1 *$

.2*

.2

.2

5.6
.0

. 3*

$.2 *$

1.0

1.5

.6

.5

.5

.4

.1

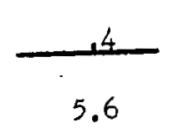

5.6
2957 Formula Best Det.

0.1

.2

.3

2.1

1.0

1.2

11.8

3.9

4.0

2.5

1.3

.2

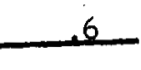

16.5

1952

Formula Best Est. Formula Best Eat.

$\begin{array}{lc}\text { Jan. } & 0.1 \\ \text { Feb. } & .3 \\ \text { March } & .2 \\ \text { April } & 1.8 \\ \text { May } & 2.2 \\ \text { June } & 1.2 \\ \text { Ju1y } & .5 \\ \text { Aug. } & 1.2 \\ \text { Sept. } & .5 \\ \text { Oct. } & 2.1 \\ \text { Nov. } & .3 \\ \text { Dec. } & -10.6 \\ \text { Total } & 10.2\end{array}$

0.4

.3

.6

1.7

3.4

2.6

.9

.5

.5

.2

Pre-1954 2.8

$1954 \quad 2.4$

$1955 \quad 5.6$

$1956 \quad 5.6$

$1957 \quad 16.5$

$1958 \quad 10.6$

$1959 \frac{11.3}{55.0}$

Total 


\section{ESTIMATE OF Sr 90 DEPOSITION FROM GUMMD FIL}

STATION \# 511

LOCATION EDMONTON, ALBERTA, CANADA

1954

1952

1256

1957

$\frac{\text { Eormula Best Est. }}{0.04} \frac{\text { Formula Best Est. }}{0.01 *} \quad \frac{\text { Formula Best Este }}{0.10 *} \quad \frac{\text { Formula Best Est. }}{0.09}$

Jen.

.02

.04

Feb.

.01

.35

Apr11 $\quad .05$

May $\quad .12$

June $\quad .06$

JuIy $\quad .13$

Aug. $\quad .14$

Sept. $\quad .09$

Oct.

.05

Nov.

.10

Dec.

.00

TOTAL

.81

.24

.50

$.23 *$

$.24 *$

$.24 *$

$.05 * *$

.10

$.25 *$

.18

.29*

.78

1.19

1.02

.83

.75

.54

.85

.23

.59

$.15 *$

1.37

$.16 *$

.31

.17

.08

.05

.10

.10

$\frac{.08}{2.29}$

$\frac{.11}{4.79}$

6.22

1958

Formula Best Est.

1959

\begin{tabular}{rrr} 
& & Formula Best Este \\
& 0.13 & 0.23 \\
.23 & .26 \\
.27 & .43 \\
1.19 & .67 \\
1.68 & 1.42 \\
1.19 & 1.78 \\
.25 & $.82^{\circ}$ \\
.44 & .39 \\
.31 & .17 \\
.32 & .11 \\
.13 & .10 \\
.16 & .07 \\
\hline 6.30 & 6.35
\end{tabular}

Jan.

Feb.

March

Apr11

May

June

July

Aug.

Sept.

oct.

Nov.

Dec.

TOTAL

6.30

6.35 
ESTIMATE OF INFINITY $y$ DOSE FROM GUMMED FILM

STATION \# 511

LOCATION EDMONTON, ALBERTA, CANADA

\begin{tabular}{|c|c|c|c|c|}
\hline & Formula Best Est. & $\frac{1955}{\text { Formula Best Est. }}$ & Eormula Best Est. & Formula Best Est. \\
\hline Jan。 & 0.0 & $0.0 *$ & $0.2 *$ & 0.2 \\
\hline Feb. & .0 & .1 & $.1 * *$ & .2 \\
\hline March & .0 & .7 & $.4 *$ & .3 \\
\hline Apr11 & .3 & .4 & $.4^{*}$ & 1.5 \\
\hline May & .6 & .9 & 1.9 & 1.7 \\
\hline June & .2 & $.4^{*}$ & 1.6 & 1.4 \\
\hline July & .4 & $.4^{*}$ & 1.2 & 1.4 \\
\hline Aug. & .4 & $.4 *$ & .5 & .9 \\
\hline Sept. & ? & $.2 *$ & 2.1 & 2.8 \\
\hline oot. & .1 & $.3 *$ & .3 & .6 \\
\hline Nov: & .2 & .1 & .2 & .2 \\
\hline Dec. & .0 & .1 & - & 2 \\
\hline TOTAL & 2.3 & 4.0 & 9.1 & 11.4 \\
\hline
\end{tabular}

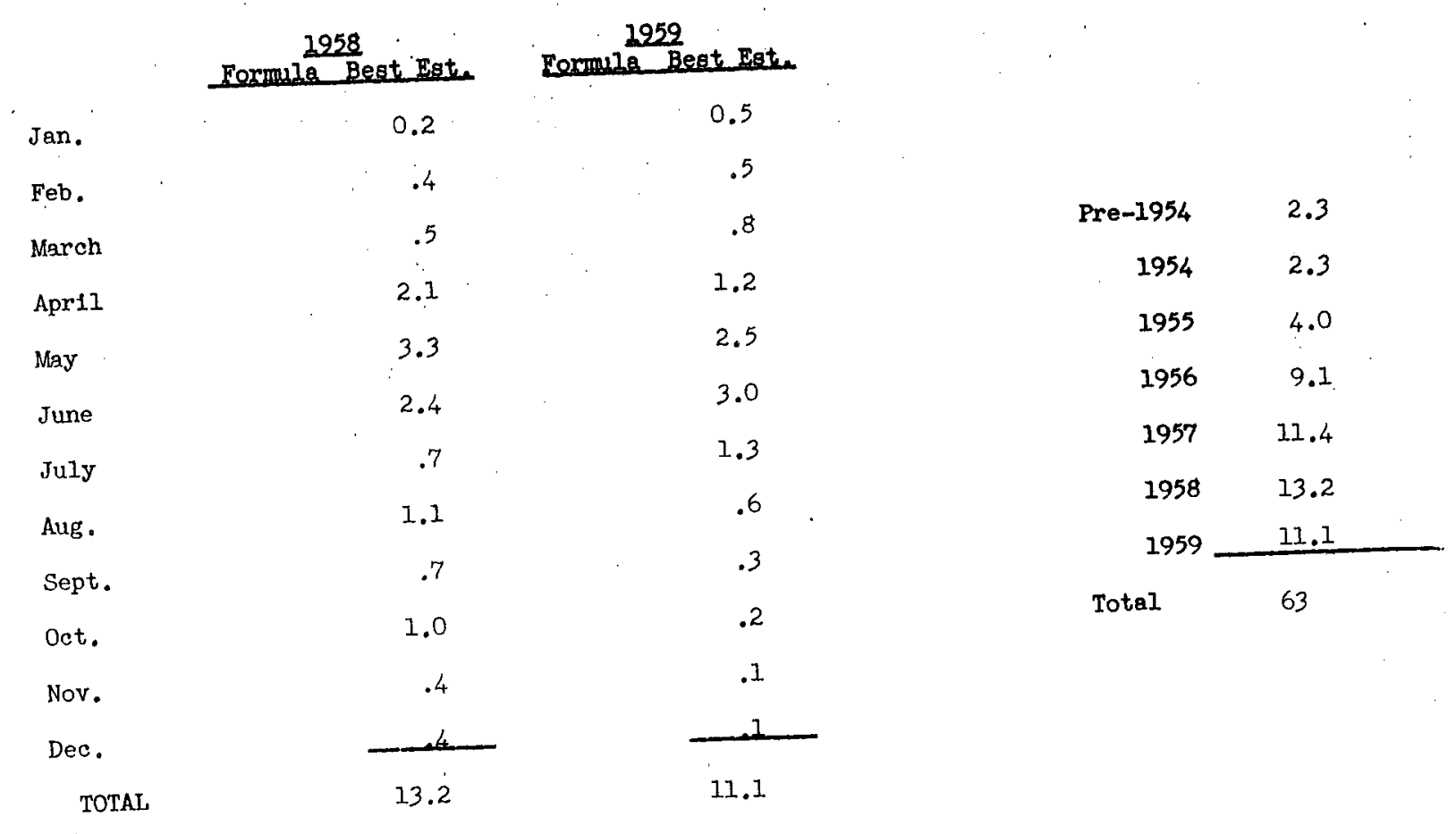




\section{ESTIMATE OF $5{ }^{90}$ DEPOSITION RRO GUMAOD FIL}

STATOON H-.515

LOCATION DEEP RIVER, ONTARIO, CANADA

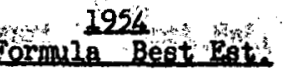

1255

1256

Eormula Best Est:

Jan.

0.04

freb.

.05

$.12 *$

Narch

.07

.20

April

May

.05

.28

$1.51 \quad .59$

Eormila Best Est.

Formi2 Best Est.

$0.11 *$

0.10

June

.05

$5.52 \quad 1.52$

.05

.10

$.12 *$

.16

Juiy

.05

.07

Aug.

.08

Sept.

.09

oct.

.07

Nov.

.07

Dea.

TOTAL

$\frac{.08}{.77}$

$.24 *$

$.67 *$

.46

.66

.39

.23

.41

$.02 *$

$.04 *$

.18

.80

.08

.21

.11

.99

.18

.08

.10

.14

.12

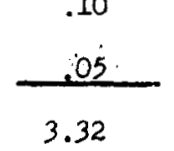

$\frac{.13}{2.66}$

.13

3.95

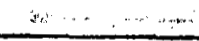

2958

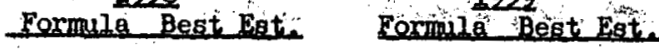

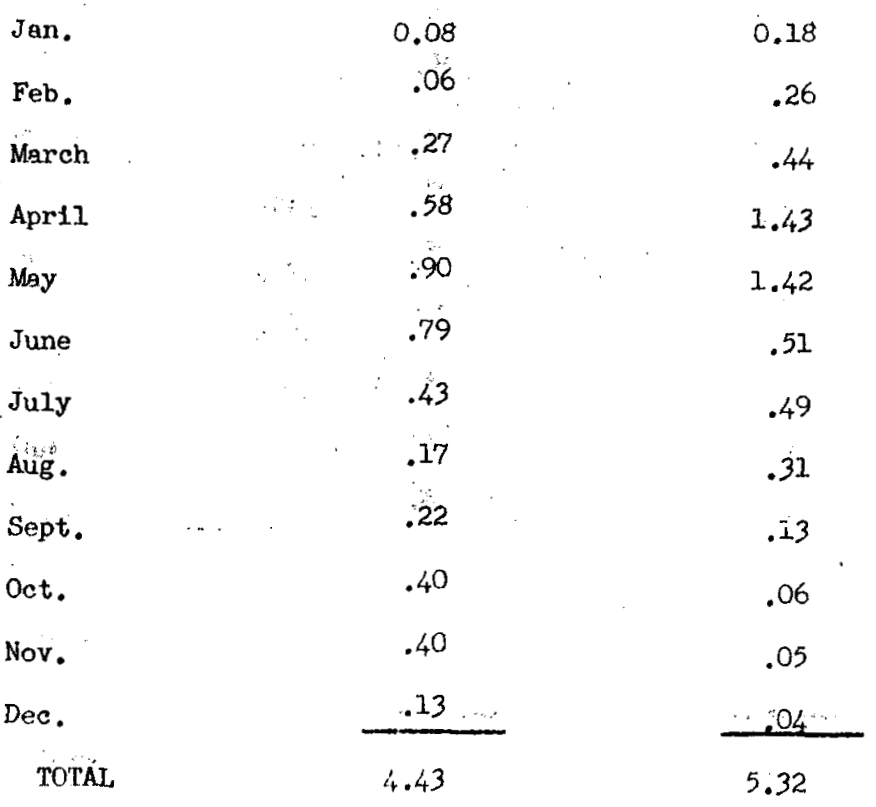

\begin{tabular}{rr} 
Pre 1954 & 0.94 \\
1954 & .77 \\
1955 & 3.32 \\
1956 & 2.66 \\
1957 & 3.95 \\
1958 & 4.43 \\
1959 & 5.32 \\
\hline Total & 21.4
\end{tabular}




\section{4} Formula Best Est.

Jan.

Feb.

March-

April

May

June

July

Aug.

Sept.

oct.

Nov.

Dec.

TOTAL
0.0

.1

.1

.3

.3

.2

.2

.1

.1

.1

.1

1.7
1955

Formula Best Est.

$.2 *$

.4

.5

2.8

1.1

9.9

2.8

$.4 *$

$.0 *$

..$*$

.L*

$7 *$

.2

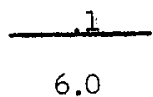

2956 Eormula Best Est.

$0.2 *$

$.1 *$

.2

$1.0 *$

1.0

.5

.4

.2

.3

.3

.3

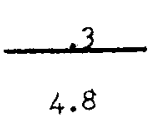

1957

Formula Best Est.

0.2

.2 .

.2

.8

.7

.7

1.3

.3

1.6

.2

.3

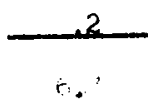

1958

Formulia Best Est.

Jan.

Feb.

March

Apr 111

May

June

July.

Aug:

Sept.

oct.

Novi..

DEC

TOTAL
0.1

.1

.5

1.0

I. . 7

1.5

$1: 1$

.4

.5

1.5

1.2

$-.3$

9.9
1959

Formula Best Est.

0.4

.5

.8

2.6

2.5

.9

.8

.5

.2

.1

Pre-1954 2.8

$1954 \quad 1.7$

19556.0

1956. $4.8^{\circ}$

1957 6.7

1958: 9.9

1959

9. 5

Total:

41.0

.1

.1

9.5 


\section{ESTIMATE OF Sr 90 DEPOSIYION FROM GUMMED FILM}

STATION \# $\quad 516$

LOCATION_GOOSE BAY, NEWFOUNDIAND, CANADA

1954

1955

1256

1957

Formula Best Esti.-. Eormula Best Este Formule Best Est. Formula Best Est.

San.

0.04

$.04 *$

$.26 *$

0.09

Féb.

.09

.04

.05

.11

March

.02

.07

$.08 *$

.18

ApriI

.01

.42

$.05 *$

.39

May

.02

.50

.50

.53

June

.07

$.43 *$

.31

.68

July

.07

$1.88 *$

.40

.55

Aug .

.08

$.56 *$

.13

.56

Sept.

.15

Oct.

.12

$.41 *$

.25

1.16

Nov.

.13

$.15 *$

.25

.29

Deo.'

.03

.09

.15

.27

TOTAL

.82

$-18$

4.77

.06

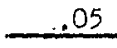

2.49

4.86

$+\quad+\cdots+\cdots$

1958

Formula Best Est. Eormila Best Est.

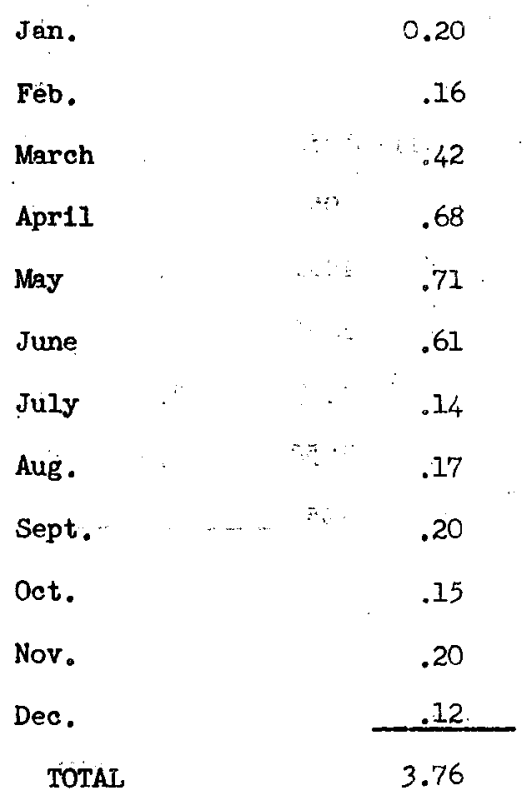

0.18

20

.23

.73

Pre-1.954 $\quad 1.08$

1.52

$1954 \quad .82$

.89

$\therefore 40$

.33

.11

$1955 \quad 4.77$

$1956 \quad 2.49$

$1957 \quad 4.86$

$1958 \quad 3.76$

.10

1959

Total 
1954<smiles>[R16][R16]</smiles>

1956 1957

Formula Best Este Formula Best Est.

Jan.

Feb.

March

April

May

June

JuIy

Aug .

Sept.

Oet.

Nov:

Dec.

TOTAL
0.0

.2

.0

.0

.1

.3

.2

.2

.4

.3

.3

.0

2.0
$.1 * *$

.1

.1

.8

.9

$.7 *$

$3.2 *$

$.9 *$

$.7 *$

$.2 *$

.2

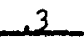

8.2
Eormula Best Est.

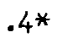

.1

$.1 *$

$.1 *$

.8

.6

.9

.3

.5

.5

.3

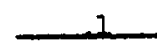

4.7
Formila Best Est.

0.2

.2

.3

.7

.9

1.2

.9

.9

1.8

.6

.6

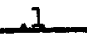

8.4
2958

Formula Beat Est.

0.3

.3

Feb.

March

Apri]

May

June

July

Aug.

Sept.

Det.

Nov.

Dec.

TOTAL
.8

1.2

1.4

1.2

.4

.4

.5

.5

.6

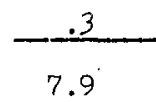

1959

Eormula Best Est.

0.4

.4

.4

1.3

2.6

1.5

.6

.5

.2

.1

\begin{tabular}{rl} 
Pre-1954 & 4.4 \\
1954 & 2.0 \\
1955 & 8.2 \\
1956 & 4.7 \\
1957 & 8.4 \\
1958 & 7.9 \\
1959 & \\
\hline
\end{tabular}

Total 
ESTIMA'SE OE Sr 90 DEPOSITION FROM GUMMED EILM

STATION \# 517

LOCATION STEPHENVILLE, NEWFOUNDLAND, CANADA

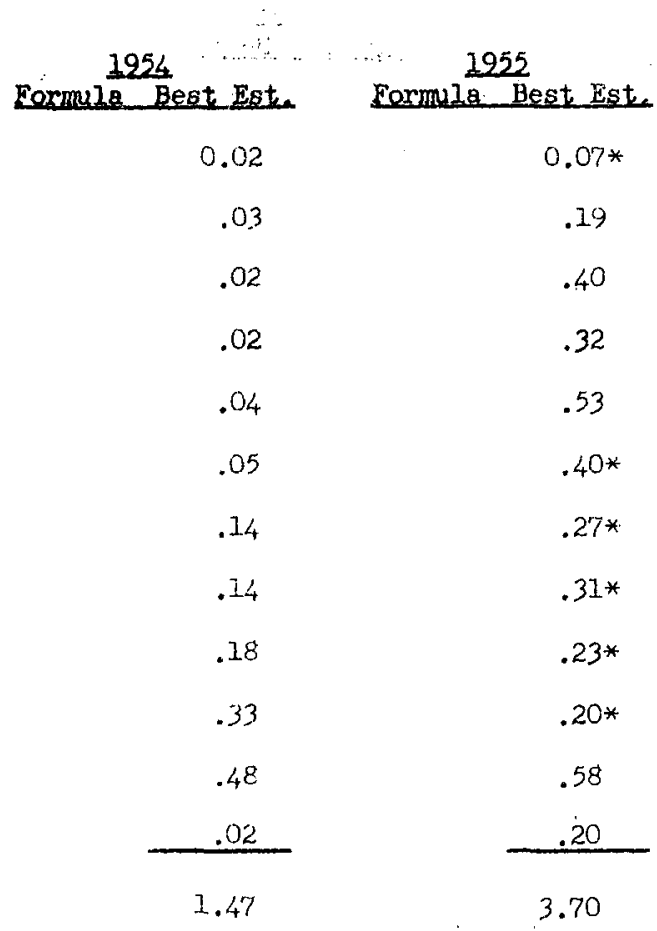

2956

1957

Formula Best Est.

$0.26 *$

.22

$.30 *$

$.48 *$

.90

.35

.14

.21

.39

.26

.32

.33

4.16
Formule Best Est.

0.19

.13

.19

1.17

.67

.54

.51

.50

1.20

.47

.22

.68

6.57

(n)

TOTAL

3.70 1952.

Formula Best Est. Formula Best Est.

$\begin{array}{lcc}\text { Jan. } & 0.23 & 0.16 \\ \text { Feb. } & .27 & .38 \\ \text { March } & .28 & .27 \\ \text { Apr11 } & .94 & .91 \\ \text { May } & .99 & 1.95 \\ \text { June. } & .55 & .67 \\ \text { Ju1y } & .19 & .61 \\ \text { Aug. } & .21 & .36 \\ \text { Sept. } & .29 & .25 \\ \text { Oct. } & .58 & \\ \text { Nov. } & .56 & \\ \text { Dec. } & .19 & \\ \text { ToTAL } & 5.28 & \end{array}$

\begin{tabular}{rr} 
Pre-1954 & 1.53 \\
1954 & 1.47 \\
1955 & 3.70 \\
1956 & 4.16 \\
1957 & 6.57 \\
1958 & 5.28 \\
1959 & \\
\hline
\end{tabular}


1254 Formula Best Est.

$\begin{array}{lc}\text { Jan. } & 0.0 \\ \text { Feb. } & .1 \\ \text { March } & .0 \\ \text { Apr1I } & .1 \\ \text { May } & .2, \\ \text { June } & .2 \\ \text { JuIy } & .4 \\ \text { Aug. } & .4 \\ \text { Sept. } & .5 \\ \text { Oct. } & .8 \\ \text { Nov. } & 1.1 \\ \text { Dec. } & .1 \\ \text { TOTAL } & 3.9\end{array}$

1955 Formula Best Este.

$0.2 *$

.4

.8

.6

1.0

$.7 *$

$.4^{*}$

$.5 *$

$.4^{*}$

$.3 *$

1.1

.3

6.7
1956

Formule Best Est.

$0.4^{*}$

.4

$.5 *$

$.7 *$

1.4

.7

.3

.5

.8

.5

.6

.6

7.4
1957 Formula Best Est.

0.3

.2

.3

2.0

1.2

1.0

.8

.9

1.9

1.0

.5

1.2

11.3

1959

Formula Best Est. Formula Best Est.

\begin{tabular}{lr} 
Jan. & 0.4 \\
Feb. & .4 \\
March & .5 \\
Apr1I & 1.6 \\
May & 1.9 \\
June & 1.1 \\
July & .5 \\
Aug. & .5 \\
Sept. & .7 \\
Oct. & 2.2 \\
Nov. & 1.6 \\
Dec. & .5 \\
\multicolumn{1}{c}{ ToTAL } & 11.9
\end{tabular}

0.3

.8

.5

1.6

3.4

1.1

1.0

.6

.4

$\begin{array}{rr}\text { Pre-1954 } & 7.9 \\ 1954 & 3.9 \\ 1955 & 6.7 \\ 1956 & 7.4 \\ 1957 & 11.3 \\ 1958 & 11.9 \\ 1959 & \\ \text { Total }\end{array}$


ESTIMATE OF Sr90 DEPOSYTYON FROM GUMMED FIIM

STATION \# 518

WOCATION THULE, GREENLAND

\section{$\therefore \quad 1954$}

1955

1256

1957

\section{Cormula Best Est}

Jan.

0.04

$0.01 *$

$0.13 *$

0.06

Feb.

.04

.17

March

Apri1

.00

.02

May

.01

.02

June

.04

July

.07

Aụg .

.05

Sept.

.04

Oct.

.04

Nov.

.00

Dec.

.03

TOTAL

.38

.09

.18

$.10 *$

$.09 *$

$.17 *$

$.13 *$

$.24^{*}$

.28

12

.11

.08

$.06 *$

.08

$.09 *$

.14

.39

.46

.23

.68

.25

1.00

.18

.57

.14

.63

.16

$.31 * *$

.05

$.14 * *$

.05

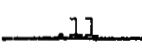

1.60

1.84

4.26

1958.

Formula Best Est.

1922 Best Este

Jan.

0.35

0.38

Feb.

.17

March

.40

April

.31

May

.37

June

.28

Ju1y

.17

Aug.

.15

Sept.

.10

Oct.

.24

.29

Nov.

.11

Dec.

TOTAI,

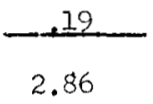
Pre-1954
0.67
$1954 \quad .38$
$1955 \quad 1.60$
19561.84
$1957 \quad 4.26$
$1958 \quad 2.86$
1959

Total 
1254 Fordulis Best Est.

\begin{tabular}{lc} 
Tan. & 0.0 \\
Feb. & .1 \\
March & .0 \\
April & .0 \\
May & .1 \\
June & .1 \\
JuIy & .2 \\
Aug. & .1 \\
Sept. & .1 \\
Oct. & .1 \\
Nov. & .0 \\
Dec. & .0 \\
\hline ToTAL & .8
\end{tabular}

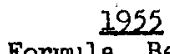

Formula Best Est.

$0.0 *$

.3

.0

.2

.3

$.2 *$

$.2 *$

$.3 *$

$.2 *$

$.4^{*}$

.4

.2

2.7
1256

Formula Best Est.

$0.2 *$

.2

$.1 *$

$.1 *$

.6

.4

.6

.4

.3

.3

.1

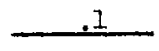

3.4
1957

Formule Best Est.

0.1

.1

.1

.3

.8

1.2

1.6

.9

1.0

$.7 * *$

$3 * *$

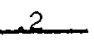

7.3

1958

Formile Best Est.

1959

Formula Best Est.

Jan.

0.6

0.8

Feb.

.3

March

.8

$\Lambda$ pril

May

.6

.7

June

.5

July

.4

Aug .

.4

Sept.

.2

Oct.

.8

.6

Nov.

.3

Dec.

TOTAL

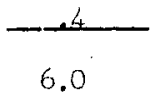

.4

.6

1.1

1.0

.9

.2

.2

.2

\begin{tabular}{rr} 
Pre-1954 & 1.0 \\
1954 & .8 \\
1955 & 2.7 \\
1956 & 3.4 \\
1957 & 7.3 \\
1958 & 6.0 \\
1959 & 1 \\
\hline
\end{tabular}

Total 
1954

Eormula Best Est.

0.04

Feb.

March

April

May

June

July

Aug .

Sept.

oct.

Nov.

Deo.

TOTAL

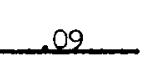

.87
1955

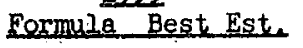

$0.03 *$

$.03 *$

.27

.87

.42

$.25 *$

$.35 *$

$.21 *$

$.17 *$

$.03 *$

.20

$36 * *$

3.19
1956

Formula Best Este Formula Best Est.

$0.34 *$

0.43

$.11 * *$

$.60 *$

$.52 *$

1.03

.64

.22

.13

.36

.34

.30

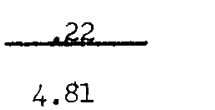

.15

.30

.84

.48

.28

.50

.47

.77

.62

4.81

1958

Jan.

Feb.

March

Apri1

May

June

July

$\begin{array}{rr}\text { Pre-1954 } & 0.90 \\ 1954 & .87 \\ 1955 & 3.19 \\ 1956 & 4.81 \\ 1957 & \\ 1958 & \end{array}$

Aug.

1959

Sept.

Total.

Oct.

Nov.

Dec.

TOTAL 
STATION \# 519

LOCATION YEFTAVIK, ICETAND

1254

1952

Formula Best Est. Eormula Best Est.

$0.1 *$

1956

1957

\begin{tabular}{lc} 
Jan. & 0.0 \\
Feb. & .1 \\
March & .2 \\
April & .0 \\
May & .1 \\
June & .1 \\
July & .2 \\
Aug. & .2 \\
Sept. & .2 \\
Oct. & .3 \\
Nov. & .7 \\
Deo. & .1 \\
\hline TOTAL & 2.2 \\
\hline
\end{tabular}

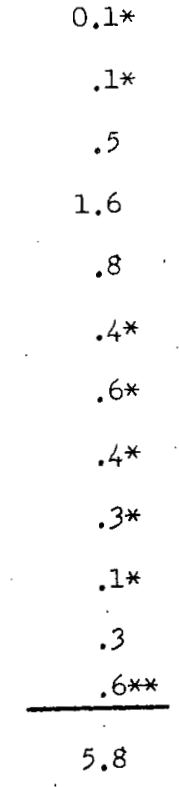

Formula Best Est.

$$
0.5 *
$$

$.2 * *$

$.9 *$

$.8 *$

1.9

1.3

.5

.3

.8

.6

.6

.4

8.8

Formula Best Est.

0.8

.2

.5

1.6

.8

.5

.8

.7

1.3

1.5

5.8

Jan,

Feb.

March

Apr11

May

June

July

Aug.

Sept.

oct.

$\begin{array}{rr}\text { Pre-1954 } & 2.0 \\ 1954 & 2.2 \\ 1955 & 5.8 \\ 1956 & 8.8 \\ 1957 & \\ 1958 & \\ 1959 & \end{array}$

Total

Nov.

Dec.

TOTAL 
522

1254

Formula Best Est.

Jan.

0.04

Feb.

March

April

May

June

JuIy

Aug.

Sept.

Dot.

Nov.

Deo.

TOTAL
.03

.02

.00

.01

.03

.06

.10

.13

.09

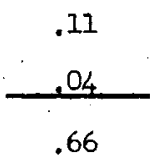

1955

Formula Best Est.

$0.02 *$

.16

.04

.02

.05

$.20 *$

$.11 *$

$.14 *$

$.13^{*}$

$.05 *$

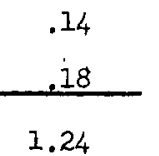

1956

Formula Best Est.

$0.09 *$

$.10 *$

.11

$.19 *$

.11

.13

.08

.14

.24

.14

.05

.15

1.53
1957

Formule Best Est.

0.16

.07

.15

.23

.36

.24

.15

.32

.67

.39

.21

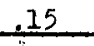

3.10

1958

Formula Best Eat.

1952

Jan.

Feb.

March

Apr11

May

June

July

Aug.

Sept.

oct.

Nov.

Bec.

TOTAI.
0.06

.12

.42

1.50

.26

.49

.15

.14

.11

.30

.16

$-.16$

3.87

Formula Best Est.

0.39

.24

.24

.36

.73

.58

.23

.24

.24

.09

Pre-1954 0.99

1954.66

$1955 \quad 1.24$

$1956 \quad 1.53$

$1957 \quad 3.10$

$1958 \quad 3.87$

$1959 . \quad 3.40$

Total 14.8 
ESTIMATE OF INFINITY Y DOSE FROM GUMED FILM

STATTON \# 522

LOCATION NOME AIASKA

1254 Formula Begit Eate.

Jan.

Feb.

March

Apri1

May

June

July

Aug.

Sept.

oct.

Nov.

Deo.

TOTAL
0.0

.1

.2

.0

.1

.1

.2

.3

.3

.2

.2

.1

1.8
1955 Formula Begt Est.

$0.0 *$

.3

.1

.0

.1

$.4 *$

$.2 *$

$.2 *$

$.2 *$

$.1 *$

.3

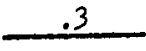

2.2
1956 Eormula Best Est.

$0.1 *$

.2

$.2 *$

$.3 *$

.2

.3

.2

.3

.5

.3

.1

3

3.0
1957

Rormula Best Est.

0.3

.1

.2

.4

.6

.4

.2

.5

1.1

.9

.5

.3

5.5
0.1

Jen.

Feb.

March

Apri1

May

June

July

Aug.

Sept.

oct.

Nov.

Dec.

TOTAL
.2

.8

2.6

.5

.9

.4

.3

.3

1.0

.5

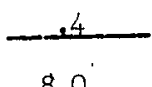

8.0
1959

Cormula Best Est.

0.8

.5

.3

.7

1.3

1.0

.4

.4

.4

.1

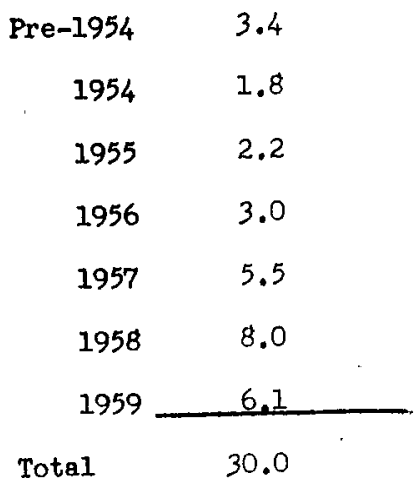

.1

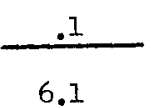




\section{ESTIMATE OF SY 90 DEPOSILION RROM GUMMED ELLM}

STATION \#_.523

LOCATION FAIRBANKS, ALASKA

1254

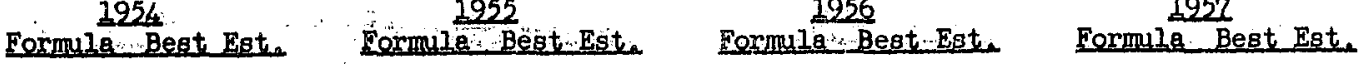

Formuli Best Est. Formule Best Esta Formula Best Est. Formula Best Est.

1957

Jan.

0.04

$0.01 *$

$0.03 *$

0.06

Feb。

.04

.04

.06

.09

March

.00

.09

April

.00

.13

Mấy

.01

.21

June

.04

$.45 *$

JuIy

.05

$.26 *$

Aug.

.05

$.27 *$

Sept.

.07

$.12 *$

oct.

.05

$.03 *$

Nov.

.06

Deo.

.04

.07

TOTAL

.04

1.74

$2.38 *$

.15

$.48 *$

.18

.47

.61

.28

.62

.10

.40

.23

.50

.32

.67

.13

.17

.01

.04

.43

$\frac{.02}{4.51}$

.11

3.60

1958

Formula Best Est. Formulia Best Est.

$\begin{array}{lcc}\text { Jan. } & 0.06 & .0 .10 \\ \text { Feb. } & .10 & .07 \\ \text { March } & .22 & .14 \\ \text { April } & .54 & .31 \\ \text { May } & .45 & .89 \\ \text { June } & .70 & .78 \\ \text { July } & .27 & . \cdots .65 \\ \text { Aug. } & .08 & .48 \\ \text { Sept. } & .16 & .19 \\ \text { Oct. } & .09 & .39 \\ \text { Nov. } & .06 & .06 \\ \text { Dec. } & -.06 & .04 \\ \text { TotAL } & 2.79 & 4.10\end{array}$

\begin{tabular}{rr} 
Pre-1954 & 0.99 \\
1954 & .43 \\
1955 & 1.74 \\
1956 & 4.51 \\
1957 & 3.60 \\
1958 & 2.79 \\
1959 & 4.10 \\
\hline Total & 18.2
\end{tabular}


1954 Formula Besit Est.

Jan.

Feb.

March

Apr11

May

June

JuIy

Aug.

Sept.

Oot.

Nov.

Dec.

TOTAL
0.0

.1

.0

.0

.1

.1

.2

.1

.2

.1

.1

.1

1.1
1955 Eocmula Best Est.

$0.0 *$

.1

.2

.2

.4

$.8 *$

$.4 *$

$.4 *$

$.2 *$

$.0 *$

.1

.1

2.9
2956 Eormula Best Est.

$0.0 *$

.1

$3.6 *$

$.7 *$

.7

.5

.2

.5

.7

.2

.0

.9

7.2
1952

Eormila Best Est.

0.1

.2

.2

.3

1.0

1.1

.6

.8

1.1

.4

.1

6.1

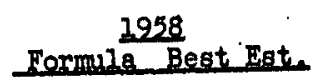

0.1

Jen.

Feb.

March

Apr11

May

June

July

Aug.

Sept.

oct.

Nov.

Dec.

TOTAL
.2

.4

1.0

.9

1.4

.7

.2

.4

.3

.2

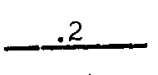

$6.0^{\circ}$

1952

Coxmula Best Eat.

0.2

.1

.3

.6

1.5

1.3

1.1

.8

.3

.6

.1

1

7.0

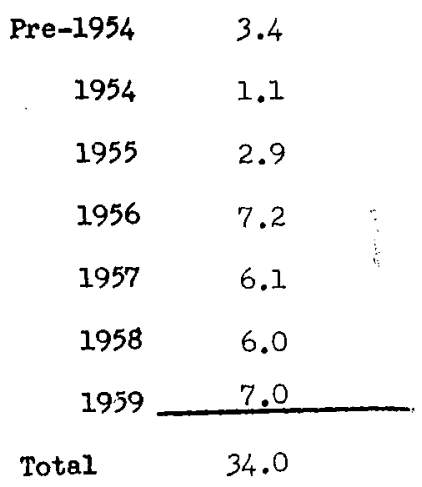


STATION \# 524

IOCATION JUNEAU, AIASKA

1924

1955

1256

1957

Eormula Best Eete Formila Best Est. Eormula Best Est. Formuli Best Est.

Jan.

0.04

$0.08 *$

$0.13 *$

0.21

Feb.

.15

.12

.31

.28

March

.00

.23

ApriI

.00

.31

$.26 *$

.29

May

.02

.61

$.44^{*}$

.63

June

.02

JuIy

.06

Aug.

.03

Sept.

.10

oct.

.23

Nov.

.30

Dec.

.04

$.19 *$

.37

.67

$8 *$

.09

.27

$.08 *$

$.22 *$

$.10 *$

$.15 *$

TOTAL

.99
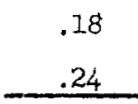

.04

.17

.21

.30

.44

.48

.25

.40

.43

.22

.46

.26

2.51

3.43

4.18

1958

Formula Best Est. Formula Best Est.

Jan.

0.53

0.55

Feb.

.36

.91

March

.62

1.52

Apr11

.61

1.14

May

.34

.96

June

.47

1.06

July

.11

.38

Aug.

.08

Sept.

.18

Oct.

.70

Nov.

.86

Dec.

TOTAL

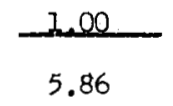

$$
5.86
$$




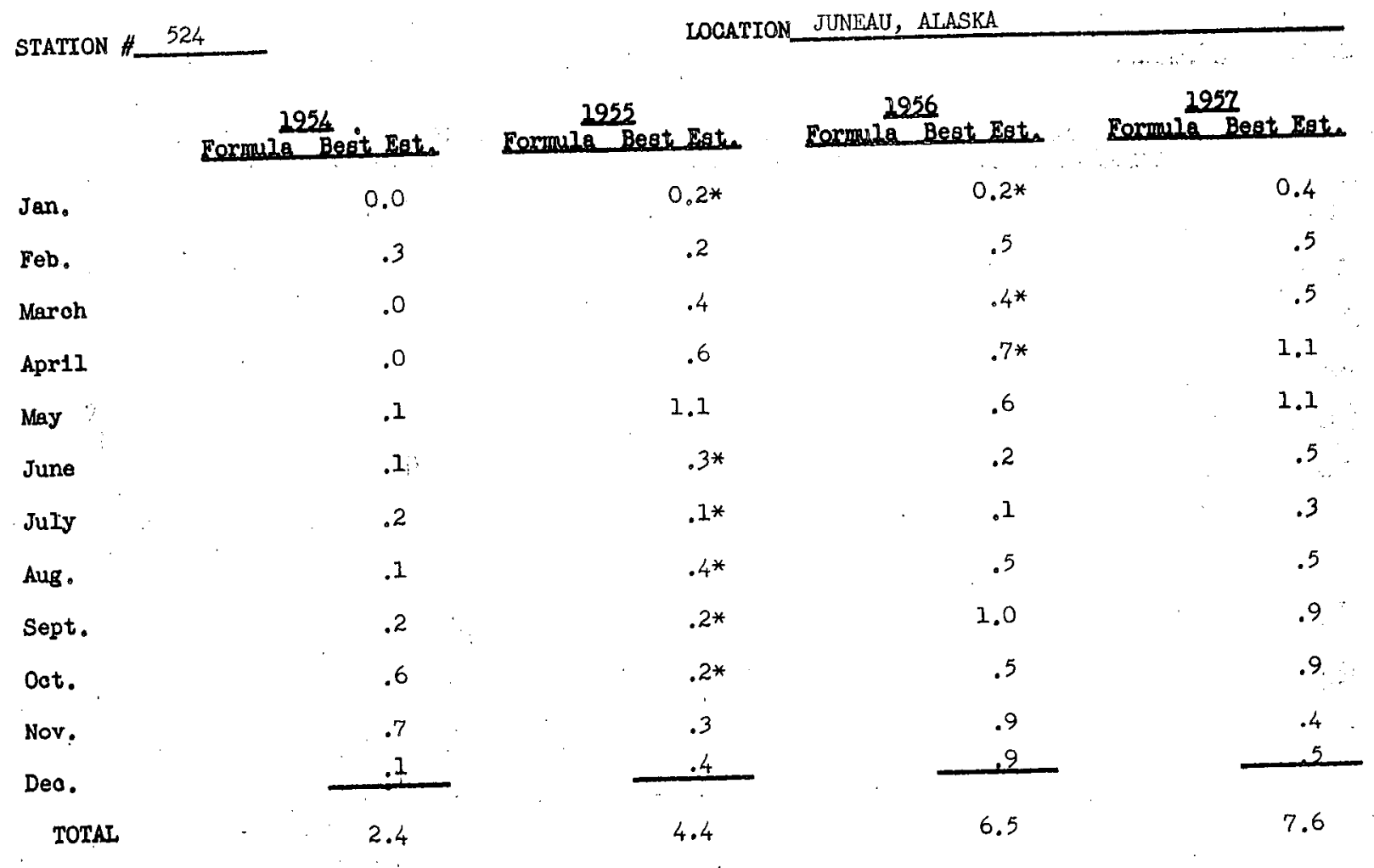

\section{Formula Best Eat. Bormula Best Esta}

$\begin{array}{lcc}\text { Jan. } & 0.9 & 1.2 \\ \text { Feb. } & .6 & 1.8 \\ \text { March } & 1.2 & 2.9 \\ \text { April } & 1.1 & 2.1 \\ \text { May } & .7 & 1.7 \\ \text { June } & .9 & 1.8 \\ \text { July } & .3 & .6 \\ \text { Aug. } & .2 & \\ \text { Sept. } & .4 & \\ \text { Oct. } & 2.5 & \\ \text { Nov. } & 2.6 & \\ \text { Dec. } & 2.4 & \\ \text { TotaL } & 13.8 & \end{array}$

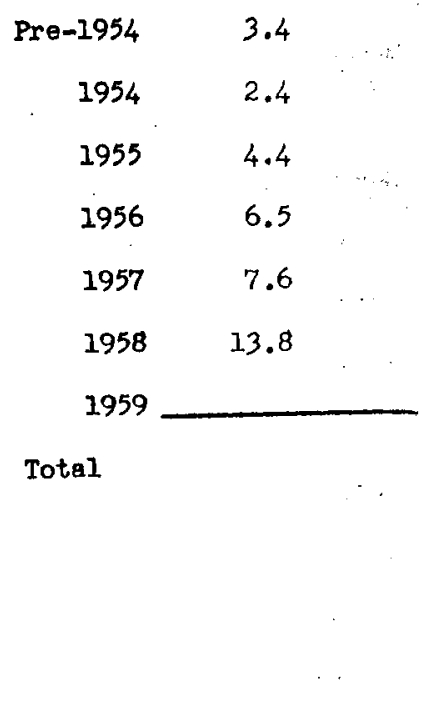


1954 0.04

Jan.

Feb,

Maroh

April

May

June

JuIy

Aug .

Sept.

Oct.

Nov.

Deo.

TOTAL
.04

.16

.05

.16

.23

.06

.11

.15

.11

.10

.02

1.23
Formula Best Est.

$.07 *$

.15

.92

.40

$1.44 \quad .82$

$.14 *$

$.09 *$

$.08 *$

$.06 *$

$.10 *$

.11

.26

3.20
1956

Eormula Best Est.

$0.25 *$

1.17

$.23 *$

$.32 *$

.54

.28

.14

.24

.15

.25

.18

.13

3.88
1957

Formula Best Est.

0.20

.08

.36

.21

.14

.21

.53

.48

.53

.14

.08

.25

3.21

1958

Formula Best Est.

Jan.

0.23

1959

Feb.

.26

March

.13

.23

April

.29

May

.33

June

.15

July

Aug.

.31

Sept.

.18

Oct.

.13

Nov.

.21

Dec.

TOTAL

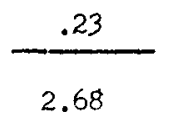

Formula Best Est.

0.60

.72

.70

.85

.73

.46

.25

.12

.11

.25

.11

.15

5.05

\begin{tabular}{rl} 
Pre-1954 & 1.03 \\
1954 & 1.23 \\
1955 & 3.20 \\
1956 & 3.88 \\
1957 & 3.21 \\
1958 & 2.68 \\
1959 & 505 \\
\hline & \\
Total & 20.3
\end{tabular}


STATION \# 525

IOCATIONSAN JUAN, PUERTO RTCO

1954 Formula Besit Est.

Jan。

Feb。

Maroh

April

May

June

July

Aug .

Sept.

Oet.

Nov.

Deo.

TOTAL
0.0

.1

.9

.3

.8

.9

.2

.3

.4

.3

.2

.1

4.5 Formula Best Est.

$.2 *$

.3

1.8

.7

2.6

1.5

$.2 *$

$.1 *$

$.1 *$

$.1 *$

$.2 *$

.2

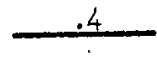

5.8
2956 Eormule Best Est.

$0.4^{*}$

1.9

$.3 *$

$.5 *$

.8

.5

.4

.5

.3

.5

.3

.2

6.6
1957

Pormula Beat Bet.

0.4

.1

.6

.4

.2

.4

.9

.7

.9

.3

.2

5.5

2952

Formula Best Est. Eomula Best Est.

\begin{tabular}{lcc} 
Jan. & 0.4 & 1.3 \\
Feb. & .4 & 1.4 \\
March & .3 & 1.3 \\
Apr11 & .4 & 1.5 \\
May & .6 & 1.3 \\
June & .6 & .8 \\
July & .4 & .4 \\
Aug. & .8 & .2 \\
Sept. & .4 & .2 \\
Oct. & .4 & .4 \\
Nov. & .6 & .2 \\
Dec. & .5 & 9.2 \\
Total & 5.8 & .2 \\
\hline
\end{tabular}

\begin{tabular}{rl} 
Pre-1954 & 3.4 \\
1954 & 4.5 \\
1955 & 5.8 \\
1956 & 6.6 \\
1957 & 5.5 \\
1958 & 5.8 \\
1959 & 9.2 \\
\hline Total & 41
\end{tabular}


1954

Eormula Best Est.

Jan.

Feb.

March

Apri1

May

June

JuIy

Aug.

Sept.

Oat.

Nov.

Deo.

TOTAL
0.04

.02

.13

.10

.16

.18

$.04 * *$

.12

.09

.05

$.08 * *$

.06

1.07
1255

Eormule Best Est.

$0.03 *$

.07

$.02 *$

$.04 *$

$.06 *$

$.02 *$

$.03 *$

$.05 *$

$.21 *$

$.05 *$

.10

.10

.78
1956 Formula Best Est. $0.08 *$

.20

$.14 *$

$.12 *$

.25

.05

.45

.31

.12

.19

.24

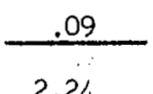

2.24
2957

Formula Best Est.

0.07

.08

.05

.06

.09

.18

.43

$.58 * *$

.15

.20

.07

.26

2.22

1958

Formula Best Est.

Jan.

0.37

Formila Best Eat.

Feb.

.06

0.18

March

.01

.12

April

.10

.10

May -

.10

June

.09

July

Aug .

.28

.39

Sept.

.26

Oct.

.21

.17

.30

$\therefore \quad .12$

.$\quad .23$

$\therefore \quad .08$

Nov.

.12

Dec.

TOTAL

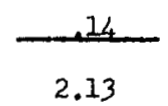

\begin{tabular}{rr} 
Pre-1954 & 0.72 \\
1954 & 1.07 \\
1955 & .78 \\
1956 & 2.24 \\
1957 & 2.22 \\
1958 & 2.13 \\
1959 & \\
\hline
\end{tabular}

Total 
STATION \# 601 Formula Besit Est.

Jen.

Feb.

March

Apr1I

May

June

Juìy

Aug.

Sept.

oct.

Nor.

Dec.

TOTAL

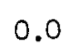

0.0

.7

.6

.8

.7

$.1 * *$

.3

.2

.1

$.2 * *$

.1

3.8

IOCATION PANAMA CANAL ZONE, ALBROOK AFB Formula Best Est.

$0.1 *$

.1

$.0 *$

$.1 *$

$.1 *$

$.0 *$

$.1 *$

$.1 *$

$.3 *$

$.2 *$

.2

.2

1.4
2956 Formula Best Est. $0.1 *$

.3

$.2 *$

$.2 *$

.4

.1

1.0

.7

.3

.3

.5

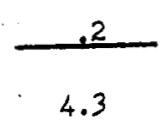

1957

Formula Best Est.

0.1

.1

.1

.1

.2

.3

$\ldots 7$

$.9 *$

.2

.4

.1

5

3.7
$J$ an.

Feb.

March

April

May

June

July

Aug.

Sept.

oct.

Nov.

Dec.

TOTAL
0.6

.1

.0

.2

.2

.2

.7

1.0

.6

.7

.3

.3

4.9

\section{9}

Formula Best Este.

0.4

.2

.2

.3

.5

.2

.4

.1

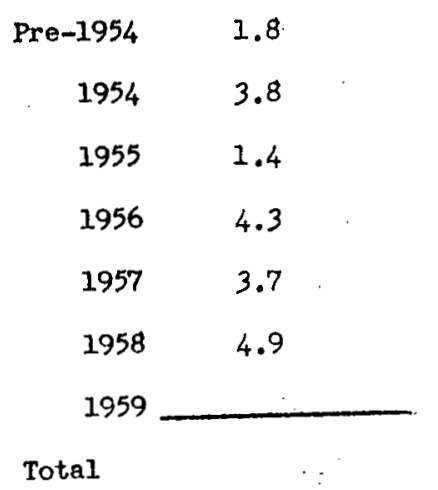


1954

Cormula. Best Est.

Jan.

Feb.

Maroh

Apr11

May

June

Juity

Aug.

Sept.

Oot.

Nor.

Deo.

TOTAL

$$
0.04
$$

.06

.07

.02

.20

.08

.07

.10

.12

.09

.27

.34

1.46
1955 Eormula Best Est.

$0.03 *$

.16

$.55 *$

$.14^{*}$

$.33 *$

$.15 *$

$.10 *$

$.08 *$

$.06 *$

$.13 *$

.23

.51

2.47
1956

Eormula Best Est. Formuli Best Est.

$0.77 *$

0.48

40

.28

$.83 *$

.67

$.31 *$

.48

.27

.42

.06

1.99

.48

.22

.40

.38

.21

.25

.27

4.50

\section{8}

1959

Formula Best Est. Eormula Best Esta

Jan.

1.14

1.49

Feb.

1.14

March

1.36

April

May

.98

.55

June

.60

July

.24

Aug.

.28

Sept.

.18

\begin{tabular}{rr} 
Pre-1954 & 1.57 \\
1954 & 1.46 \\
1955 & 2.47 \\
1956 & 4.50 \\
1957 & \\
1958 & \\
1959 & \\
\hline
\end{tabular}

Oct.

Total

Nov.

Dec.

1.28

TOTAL 


\section{ESTIMATE OF INFINITY $y$ DOSE FROM GUMMED FIIM}

STATION \# 602

IOGATION BERMUDA

1954

Besit Xat. Eormula Bet. Est.

Pomula $\frac{2956}{8}$

$0.1 *$

Jan.

$$
0.0
$$

.3

Peb.

Maroh

Apr11

.1

.4

1. $0 *$

.1

May

1.1

June

JuIy

Aug.

Sept.

Oot.

Nov.

Deo.

TOTAL

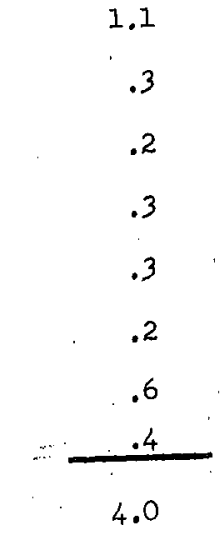

$.3 *$

$.6 *$

:3*

$.2 *$

$.1 *$

.1*

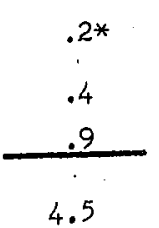

$1.2 *$

.7

$1.3 *$

$.5 *$

.4

1957

Dormiza Beat Eet.

0.8

.5

1.1

.9

.7

.1

3.6

1.0

.5

.9

.7

.2

5

.5

8.0

1958

Forming Best Eat.

1.9

Jan.

Feb.

Maroh

Apr11

May

June

July

Aug .

Sept.

Dot.

Nov.

Dec.

TOTAL
1952

Formu2a Beat Eat.

$$
3.2
$$

2.3

2.6

1.8

1.0

1.0

.4

.4

.3

\begin{tabular}{rr} 
Pre-1954 & 8.3 \\
1954 & 4.0 \\
1955 & 4.5 \\
1956 & 8.0 \\
1957 & \\
1958 & \\
1959 & \\
\hline
\end{tabular}

Totel 


\section{ESTIMATE OF Sr 90 DEPOSITION FROM GUMMED FILM}

STATTON \# 603

LOCATION IIMA, PERU

\section{Eormula Best Est.}

Jan.

Feb.

March

April

May

June

July

Aug.

Sept.

Oat.

Nov.

Deo.

TOTAL

$$
0.04
$$

$.04 * *$

.03

.07

.04

.03

.07

.05

.08

.10

09

.01

.65
2955

Cormula Best Este

$0.07 *$

.04

$.07 *$

$.09 *$

$.07 *$

$.02 *$

$.04 *$

$.06 *$

$.13 *$

$.11 * *$

$.07 * *$

$.10 * *$

.87
1256

Eormula Best Est.

$0.03 *$

$.10^{\circ}$

$.18 *$

$.12 *$

.03

.08

.08

.19

.17

.19

.09

.02

1.29
1957

Formu18 Best Est.

0.07

$.08 * *$

.07

.08

.08

.11

.14

.13

.20

.13

.22

.25

1.46

1958

1959

Formula Best Est. Eormula Best Est.

Jan.

0.07

0.06

Feb.

$.15 * *$

.06

March

.02

.03

Apr11

.02

.09

May

.04

.15

June

.11

July

.18

Aug.

.13

Sept.

.19

Oct.

.18

Nov.

.05

Dec.

$.07 * *$

TOTAL

1.21 
ESTIMATE OF INFINITY $y$ DOSE FROM GUMMED FILM

STATION \#-603

IOCATION LIMA, PERU

1954

Formula Best Est.

Jan.

0.0

Feb.

March

April

May

June

July

Aug .

Sept.

Oct.

Nov.

Dec.

TOTAL

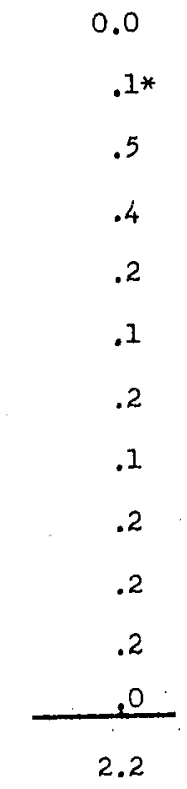

.5

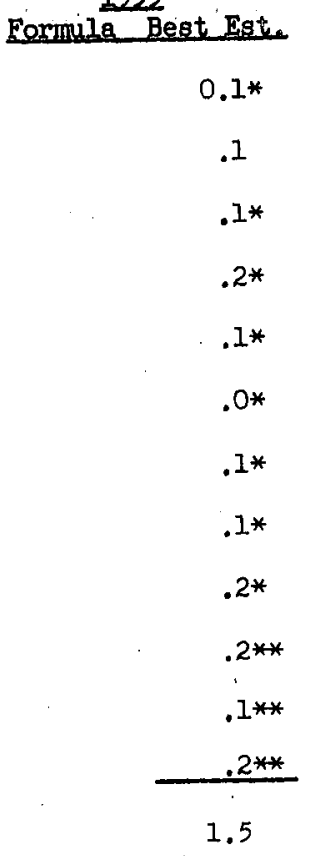

1956 Formula Best Esta.

$0.0 *$

0.1

.2

$.2 * *$

$.3 *$

.1

$.2 *$

.1

.1

.1

.2

.2

.2

.2

.4

.2

.4

$.4 \quad .3$

.2

.5

.0

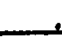

2.6

2.7

1958

1959

Formula Best Est. Formula Best Est.

$\begin{array}{lc}\text { Jan. } & 0.1 \\ \text { Feb. } & .3 * * \\ \text { March } & .0 \\ \text { Apri1 } & .0 \\ \text { May } & .1 \\ \text { June } & .2 \\ \text { July } & .5 \\ \text { Aug. } & .3 \\ \text { Sept. } & .4 \\ \text { Oct. } & .5 \\ \text { Nov. } & .2 \\ \text { Dec. } & .2^{* *} \\ \text { TotaL } & 2.8\end{array}$

0.1

.1

.0

.2

.3

2.2

$1955 \quad 1.5$

$1956 \quad 2.6$

$1957 \quad 2.7$

$1958 \quad 2.8$

1959

Total

\begin{tabular}{rr} 
Pre-1954 & $3.7 * *$ \\
1954 & 2.2 \\
1955 & 1.5 \\
1956 & 2.6 \\
1957 & 2.7 \\
1958 & 2.8 \\
1959 & \\
\hline
\end{tabular}




\section{ESTTMATE OF $\mathrm{Sr}^{90}$ DEPOSITION FRQM GUMMED FILM}

STATION \#\# 604

LOCATION SAN JOSE, COSTA RICA

\section{6}

Formula Best Est.

1955

Formula Best Est.

$\frac{1256}{\text { Formula Best Est. }}$

Formulie Best Est.

Jan.

0.04

$0.00 *$

$0.07 *$

0.04

Feb.

.02

.03

March

.16

ApriI

.05

May

.15

June

.28

July

.07

Aug.

.14

Sept.

.10

oct.

.10

Nov.

.07

$.04 *$

.12

.05

Dec.

TOTAL

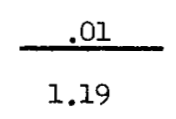

$.02 *$

$.05 *$

$.04 *$

$.02 *$

$.04 *$

$.05 *$

$.11 *$

.07

$.02 *$

.06

.11

.12

.09

.20

.23

.31

.31

.30

.12

.28

$.03 *$

.02

.14

.09

.05

.06

.07

$\frac{.05}{1.42}$

1259

Formula Best Este Formila Best Est.

Jan.

0.27

0.05

Feb.

.14

.15

March

.07

.13

April

.23

May

.21

July

.52

.12

.13

.21

.15

.19

$$
\begin{array}{rr}
\text { Pre-1954 } & 0.84 \\
1954 & 1.19 \\
1955 & .43 \\
1956 & 1.42 \\
1957 & 1.89 \\
1958 & 2.99
\end{array}
$$

Aug .

.53

Sept.

.24

oct.

.15

Total

Nov.

.06

Dec.

TOTAL

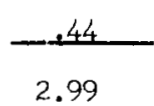


STATION \# 604

2254 Formula Best Est.

Jan。

0.0

Feb.

March

Apr11

May

June

July

Aug .

Sept.

Oot.

Nov.

Deo.

TOTAL

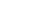

1955 Cormula Best Est.

$0.0 *$

.1

$.1 *$

$.0 *$

$.1 *$

$.1 *$

$.0 *$

$.1 *$

$.1 *$

$.0 *$

.0

.1

0.7
1956

Cermula Best Eat.

$0.1 *$

Cormula Best Est.

0.1

.2

.1

$.2 *$

.1

$.0 *$

.1

.2

.2

.2

.4

.5

.5

.7

.5

.3

.3

.4

.1

.1

2.9

3.3 $\frac{1058}{102}$ Formula Best Est.

$\therefore n$,

Feb.

March

Apri1

May

June

July

Aug.

Sept.

Oct.

Nov.

Dec.

TOTAL $\therefore .4$

.2

.1

.2

.4

.4

1.4

1.3

.5

.6

.2

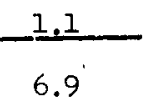

1959

Formula Best Eak.

0.1

.3

.2

.2

.4

.3

.3

\begin{tabular}{rr} 
Pre-1954 & 3.7 \\
1954 & 4.2 \\
1955 & 0.7 \\
1956 & 2.9 \\
1957 & 3.3 \\
1958 & 6.9 \\
1959 & \\
\hline
\end{tabular}

Total 


\section{ESTIMATE OF Sr 90 DEPOSITION FROM GUMMED FILM}

STATION \# 605

LOCATION IAGENS, AZORES

Cormule Best Est.

Jan.

Feb.

March

Apri2

May

June

July

Aug .

Sept.

Oat.

Nov.

Deo.

TOTAL
0.04

.06

.03

.04

.02

.07

.05

.13

.12

.19

.13

.30

1.18
1955

Fermula Best Est.

$0.04 *$

$.08 \%$

$.18 *$

$.24 *$

$.04 *$

.39*

$.01 *$

$.15 *$

$.12 *$

$.10 *$

$.22 * *$

$.52 * *$

2.09
1256

Formula Best Est.

$0.21 *$

$.64 * *$

$1.47 *$

$.54^{*}$

.55

.16

.22

.26

.26

.40

.13

.42

5.26
1927

Formula Best Est.

0.36

.41

.68

.29

.25

.39

.98

.40

.42

.28

.19

.24

4.85

1959

Formula Best Est. Formila Best Est.

Jen.

Feb.

March

April

May

June

July

Aug.

Sept.

oct.

Nov.

Dec.

TOTAL
0.25

.31

1.22

.88

.50

.54

.19

.18

.21

.25

.45

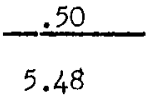

1.54

1.05

2.03

1.02

1.72

.80

.46

.45

.33

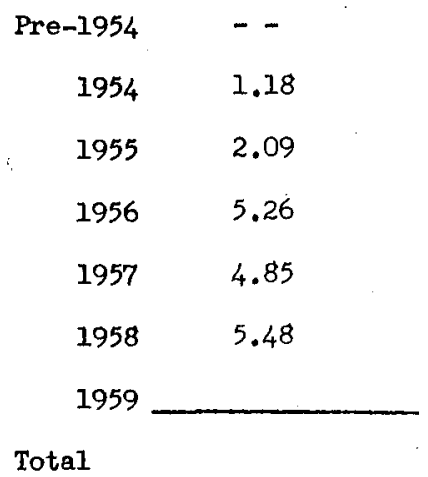


STATION \# 605

IOCATION IAAENS, AZORES

1054

1955

1921 Cormula Best Eat.

Jan.

0.0 Formula Best Eist.

$0.1^{*}$

Eormula Best Est.

Eormula Best Eat.

Maroh

.1

April

.2

May

.3

June

$$
.1
$$

.3

July

.1

Aug.

.4

Sept.

.3

oot.

.5

Nov.

.3

Deo.

TOTAL

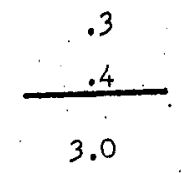

$.2 *$

.3*

$.4 *$

. $1 *$

$.7 *$

$.1 *$

$.2 *$

$.2 *$

$.2 *$

$.4 * *$

$.9 * *$

3.8

\section{$0.3 *$}

0.6

$1.1 * *$

.7

$2.2 *$

1.1

$.8 *$

.5

.9

.4

.3

.7

.5

1.6

.6

.6

.7 :

.6

.8

.3

8

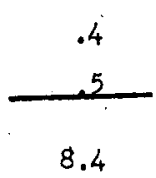

9.2

1958

Formule Best Est.

0.4

Jan.

Feb.

March

Apri1

May

June

July

Aug.

Sept.

Oct.

Nov.

Dec.

TOTAL
.5

2.4

1.6

1.0

1.0

.5

.5

.5

.9

1.3

1.2

11.8
2959

Cormula Best Eat.

3.3

2.1

3.9

1.8

3.0

1.3

.7

.7

.5

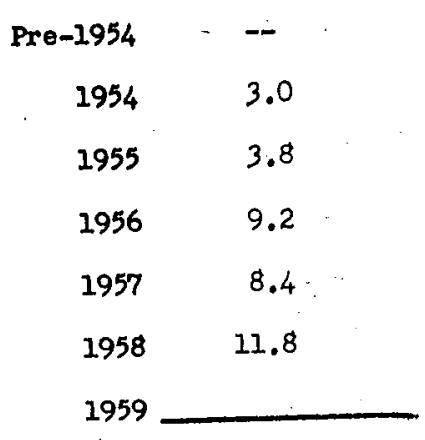

Total 
1254

Formula Best Est.

Jan.

Feb.

March

Apr11

May

June

July

Aug.

Sept.

Oct.

Nov.

Dec.

TOTAL
0.04

.07

.04

.01

.03

.02

.04

.04

.07

.10

.15

.01

.62
1955

Formula Best Est.

$0.06 *$

.10

$.05 *$

$.04 *$

$.20 *$

$.04 *$

$.03 *$

$.04 *$

.$\therefore 8 *$

$.16 *$

.13

.16

1.19
1956

Formula Best Est.

$0.23 *$

Formula Best Est.

0.13

.06

.11

$.54 *$

.07

$.08 *$

.05

.13

.16

.20

.21

.15

.09

.15

.12

.18

1.52

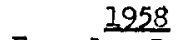

Formula Best Est. Formula Best Est.

Jan.

0.15

0.13

Feb.

.13

.21

March

.04

.09

Apri1

.03

.11

May

.11

June

.10

JuIy

.29

.10

.11

.12

Aug.

.18

Sept.

.14

Oct.

.13

Nov.

.15

Dec.

.16

TOTAL

1.61

\begin{tabular}{rr} 
Pre-1954 & 0.89 \\
1954 & .62 \\
1955 & 1.19 \\
1956 & 1.91 \\
1957 & 1.52 \\
1958 & 1.61 \\
1959 & \\
\hline
\end{tabular}

Total 
1254. Formula Best Eat.

\begin{tabular}{lc} 
Jan. & 0.0 \\
Feb. & .1 \\
March & .2 \\
April & .0 \\
May & .1 \\
June & .1 \\
July & .1 \\
Aug. & .1 \\
Sept. & .2 \\
Oot. & .2 \\
Nov. & .3 \\
Deo. & .0 \\
Total & 1.4 \\
\hline
\end{tabular}

1955

Sormula Best Est.

$0.1 *$

.2

.1*

$.1 *$

$.4^{*}$

$.2 *$

$.1 *$

$.1 *$

$.3 *$

$.2 *$

.2

.3

2.1
2956 Exmula Best Est.

$$
0.4^{*}
$$

.1

$.8 *$

$.1 *$

.2

.1

.1

.5

.3

.2

.3

.2

3.2
2957

Formula Beat Este

0.2

.2

.1

.1

.3

.2

.3

.2

.1

.3

.2

.3

2.5

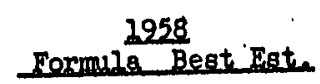

0.3

Jan.

Feb.

March

Apr1.1

May .

June

July

Aug.

Sept.

oct.

Nov.

Deo.

TOTAL

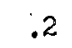

.1

.0

.2

.2

.8

.5

.3

.3

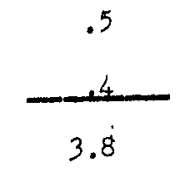

1952

Formula Best Eate

0.3

.2

.2

.2

.2

.2

.2

$\begin{array}{rr}\text { Pre-1954 } & 3.8 \\ 1954 & 1.4 \\ 1955 & 2.1 \\ 1956 & 3.2 \\ 1957 & 2.5 \\ 1958 & 3.8\end{array}$

1959

Total 
LOCATION SAO PAULO, BRAZIL

\section{Cormula Best Est.}

Jan.

Fèb.

March

Apri1

May

June

JuTy

Aug.

Sept.

oct.

Nov.

Dec.

TOTAL
.04

.05

.03

.03

.15

.10

.03

.10

.10

.11

.04

.01

.79
1955

Eermule Best Est.

$0.06 *$

$.06 *$

$.06 *$

$.03 *$

$.15 *$

$.03 *$

$.05 *$

$.06 *$

$.05 *$

$.12 *$

.12

.04

.83
1256

Eormula Best Est.

$0.11 *$

.04

$.22 *$

$.07 *$

.02

.13

.08

.12

.22

.11

$.09 * *$

.09

1.30
1957

Formula Best Est.

0.16

.10

.08

.05

.06

.20

.21

.02

.09 


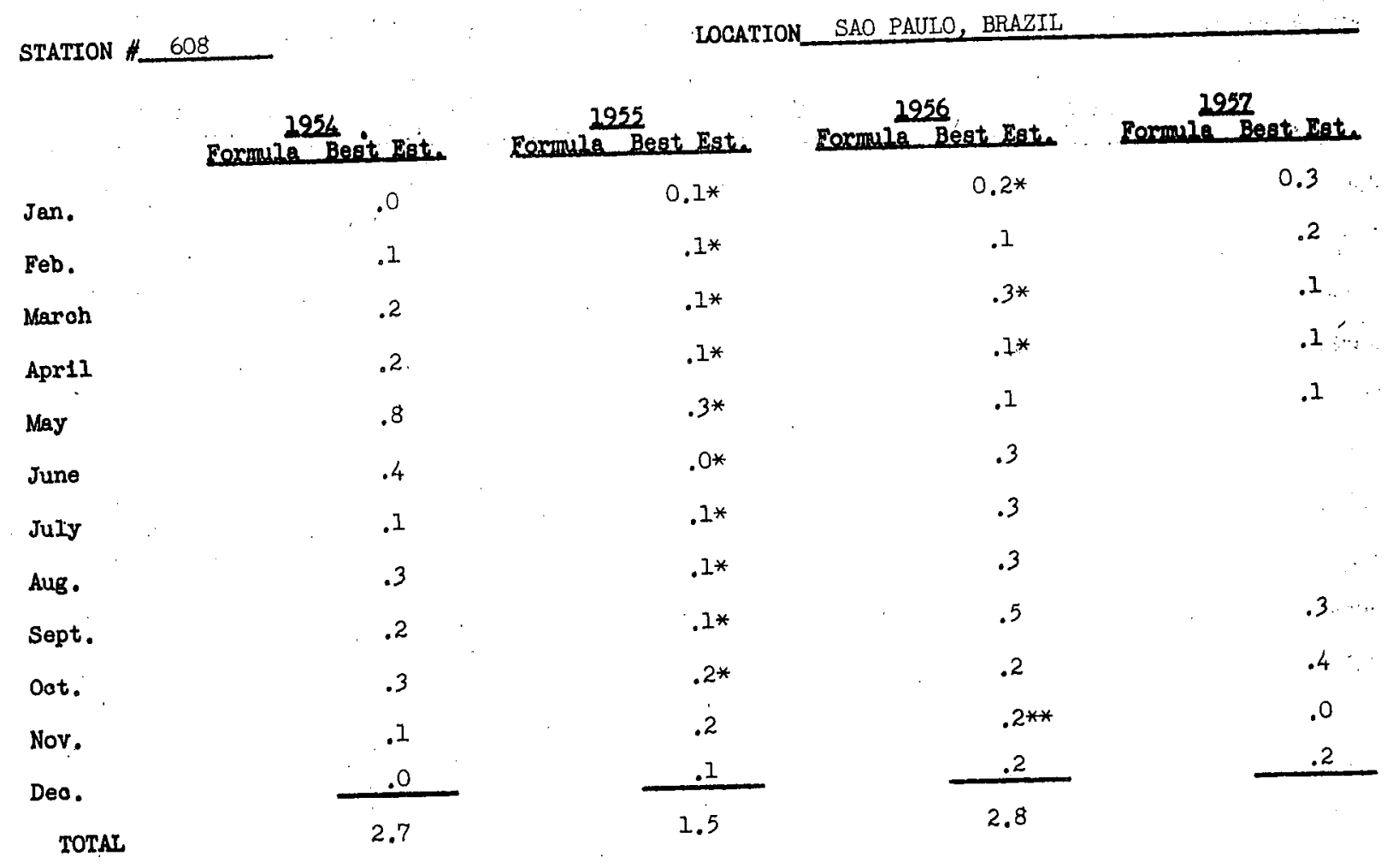

Jan.

Feb.

Maroh

April

May

June

July

Aug.

Sept.

Oot.

Nov.

Deo.

TOTAL
0.1

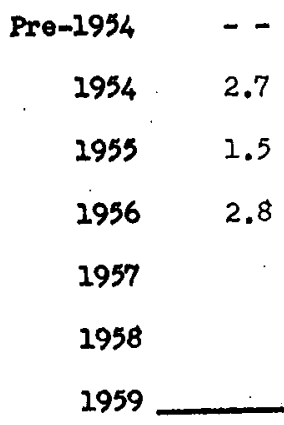

Totel 
ESTTMATE OF Sr90 DEPOSITHON EROM GUMAOD FIUX

STATION \# 611

roaATION BELEM, BRAZIL

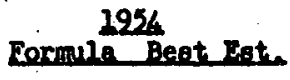

0.04

Jan.

Feb.

March

Apr11

May

June

JuIy

Aug .

Sept.

Oot:

Nov.

Dec.

TOTAL
.06

.04

.08

.27

.18

.09

.06

.25

.21

.05

.01

1.34
1955

Eormula Best Este Eocmule Hest Esta

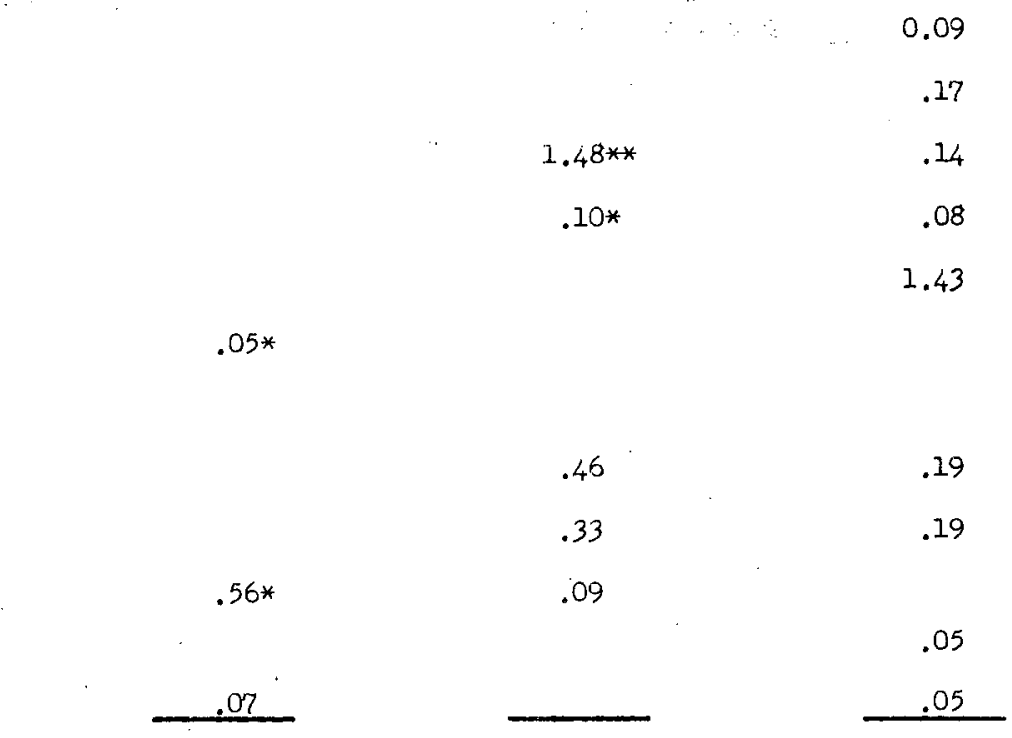

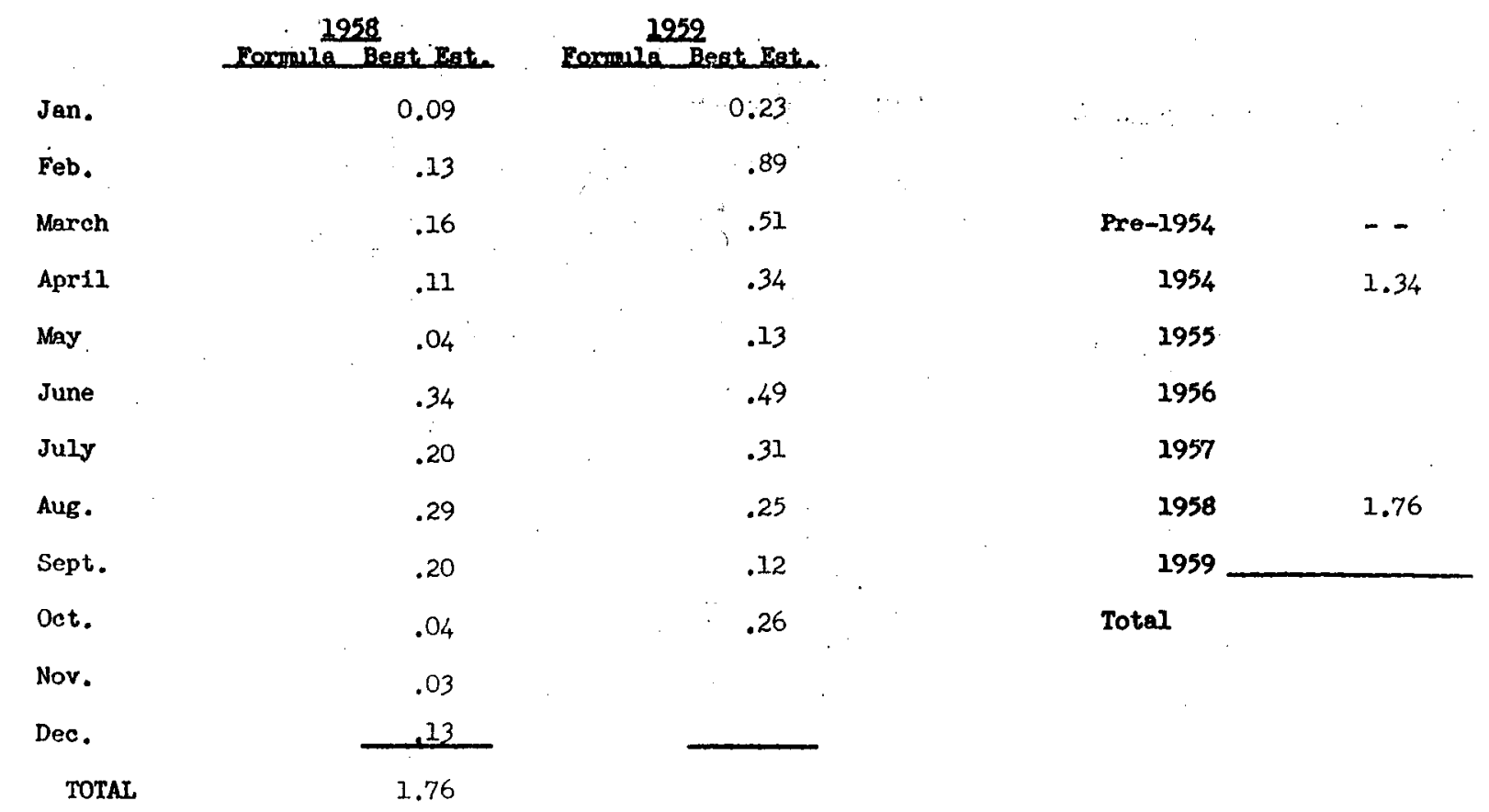




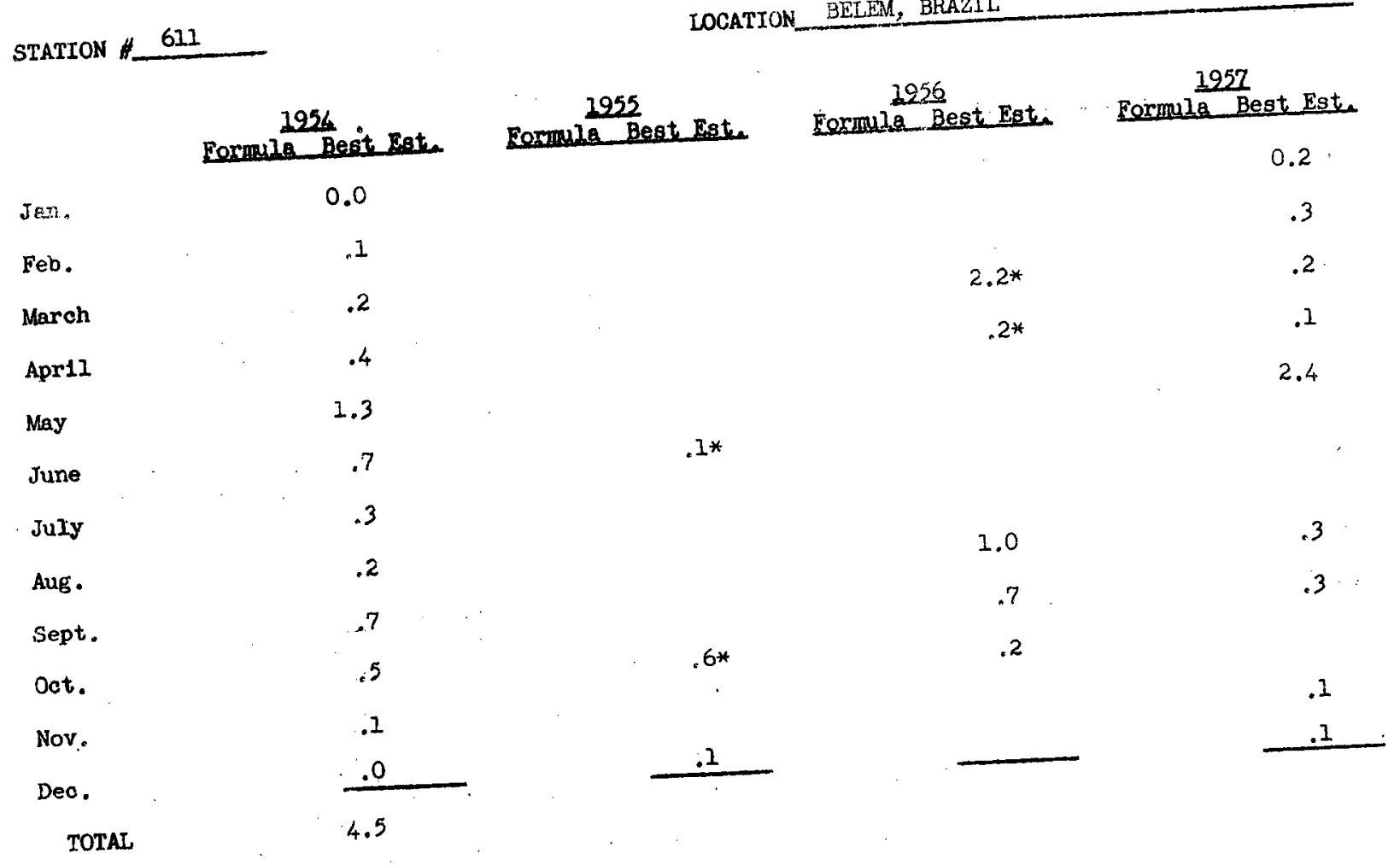

Formula Begt Est. $\frac{1958}{1259}$

$\begin{array}{lc}\text { Jan. } & 0.2 \\ \text { Feb. } & .2 \\ \text { March } & .3 \\ \text { April } & .2 \\ \text { May } & .1 \\ \text { June } & .6 \\ \text { July } & .5 \\ \text { Nug. } & .7 \\ \text { Sept. } & .5 \\ \text { Oct. } & .3 \\ \text { Nov. } & .3 \\ \text { Dec. } & 3.8 \\ \text { Total } & \end{array}$

0.5

1.8

1.0

.6

.2

.8

.5

.4

2

4

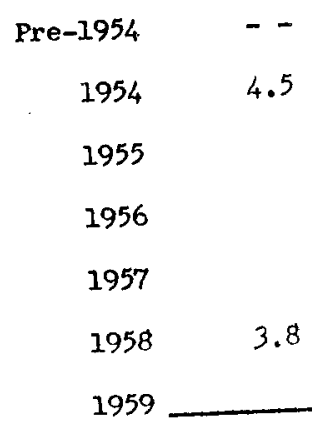

Total 


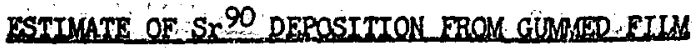

STATTON \# 612 .

LOOATION LA PAZ, BOLIVIA

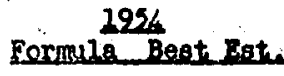

Jen.

Feb.

Maroh

Apr11

May

June

July

Aug.

Sept.

Oot.

Nov.

Dec.

TOTAL
0.04

.04

.10

.08

.45

.08

.07

.34

.48

.21

.42

.01

2.32
1955 Poxmita Best Est.

$0.19 *$

.06

$.04 *$

$.03 *$

$.30 *$

$.04 *$

$.04 *$

$.04 * *$

$.09 *$

$.23 *$

.13

.09

1.28

1956

1957

Formula Best Est. Formil Best Est.

$0.06 *$

0.10

.05

.11

$.12 *$

.04

$.05 *$

.04

.08

.35

.03

.10

.26

.08

.23

.49

.31

.23

.11

.05

.08

.06

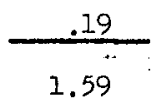

1.76

Jan.

Feb.

0.09

March

.18

.06

.08

Apr11

May

June

July

Aug .

Sept.

Oct.

Nov.

Dec.

TOTAI
1959

Pocmule Best Eat.

0.07

.08

.03

.05

.09

.08

.39

\begin{tabular}{rr} 
Pre-1954 & 0.90 \\
1954 & 2.32 \\
1955 & 1.28 \\
1956 & 1.59 \\
1957 & 1.76 \\
1958 & 2.29 \\
1959 & \\
\hline
\end{tabular}

Total 


\section{4}

Formula Best Est. Formula Best Est.

Jan.

0.0

$0.4^{*}$

Feb.

March

April

May

June

JuIy

Aug.

Sept.

Oet.

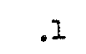

.1

.5

.4

2.5

.3

.2

.5

Nov.

, 5

Dec.

TOTAL

.1 .2

1.0

.0

7.2

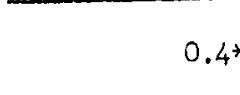

.1*

$.1 *$

$.5 *$

.1*

$.1 *$

$.1 * *$

.1*

$.4^{*}$

.2

1956

1952

Cormula Best Est.

Formula Best Est.

$0.1 *$

0.1

.1

.2

$.2 *$

. I*

.1

.1

.5

.5

.1

.6

.6

.2

.1

.8

.7

.3

.2

.I

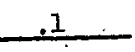

2.3

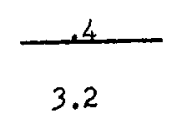

.1

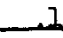

3.1

1958

Formula Best Est.

Jan.

Feb.

March

Aprt1

May

June

July

Aug .

Sept.

Oct.

Nov.

Dec .

TOTAL
0.1

.3

.1

.2

.1

.3

.6

.4

.8

1.8

.5

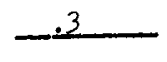

5.5
1959

Formula Best Est.

0.2

.2

.1

.1

.2

.1

.6

$\begin{array}{rr}\text { Pre-1954 } & 3.8 \\ 1954 & 7.2 \\ 1955 & 2.3 \\ 1956 & 3.2 \\ 1957 & 3.1 \\ 1958 & 5.5\end{array}$

1959

Totel 
STATTON \# 613

1954

Tormuln Best Int.

Jan.

Feb。

Maroh

April

May

June

July

Aug.

Sept.

oot:

Nov.

Deo.

TOTAL
0.04

.04

.11

.20

.13

.04

.03

.03

.10

.08

.01

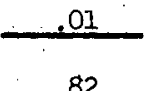

.82

LOATIOK QUITO, ECUADOR

1955

Eormule Best Est.

$0.07 *$

.12

$.02 *$

$.01 *$

$.14 *$

$.01 *$

$.02 *$

$.03 *$

$.08 *$

$.02 *$

.02

.07

.61
1956

Eormula Best Est.

$0.04 *$

$.03 \quad .04$

$.06 *$

$.02 *$

.00

.00

.02

.08

.11

.08

.09

.09

.62
1957

Eormuls Best Est.

0.05

.06

.04

.19

.08

.07

.13

.18

.10

.08

.07

1.09

2958

Formula Best Eat. Cormule Best Eat.

$\begin{array}{lcc}\text { Jan. } & 0.44 & 0.09 \\ \text { Feb. } & .09 & .15 \\ \text { March } & .10 & .08 \\ \text { Apr11 } & .02 & .07 \\ \text { May. } & .05 & .06 \\ \text { June } & .06 & .10 \\ \text { July } & .07 & .11 \\ \text { Aug. } & .09 & .06 \\ \text { Sept. } & .13 & \\ \text { Oct. } & .24 & \\ \text { Nov. } & .03 & \\ \text { Deo. } & .03 & \\ \text { Torat } & & 1.35\end{array}$

\begin{tabular}{rr} 
Pre-1954 & 0.84 \\
1954 & .82 \\
1955 & .61 \\
1956 & .62 \\
1957 & 1.09 \\
1958 & 1.35 \\
1959 & \\
\hline
\end{tabular}

Total 


$$
\text { STATION \# } 613
$$

1254 Formula Best Este

Jan,

$\mathbb{E} \in b$ :

March

April

May

June

July

Aug.

Sept.

oct.

Nov.

Dec.

TOTAL

$$
0.0
$$

.1

.6

1.1

.7

.1

.1

.1

.3

.2

.0

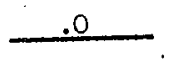

3.3

IOCATION

QUIFO, ECUADOR

1955 Formula Best Est.

$0.4 *$

.2

$.0 *$

$.0 *$

.3*

$.0 *$

$.0 *$

$.0 *$

. .

$.0 *$

.0

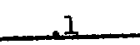

1.1

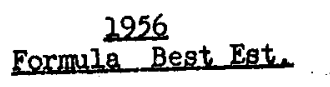

$0.1 *$

.1

$.1 *$

$.0 *$

.0

.0

.1

.2

.3

.2

.2

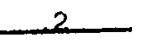

1.5
1957

Formula Best Est.

0.1

.1

.1

.1

.3

.I

.1

.2

.3

.2

.2

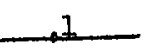

1.9

1952

Formula Best Este. Formula Best Est.

$\begin{array}{lcc}\text { Jan. } & 0.7 & 0.2 \\ \text { Feb. } & .1 & .3 \\ \text { March } & .2 & 2 \\ \text { Apri1 } & .0 & 1 \\ \text { May } & .1 & .1 \\ \text { June } & .1 & .2 \\ \text { July } & .2 & .2 \\ \text { Aug. } & .2 & \\ \text { Sept. } & .3 & \\ \text { Oct. } & .6 & \\ \text { Nov. } & .1 & \\ \text { Dee. } & -1 & \\ \text { TOTAL } & 2.7 & \end{array}$

\begin{tabular}{rr} 
Pre-1954 & 3.6 \\
1954 & 3.3 \\
1955 & 1.1 \\
1956 & 1.5 \\
1957 & 1.9 \\
1958 & 2.7 \\
1959 & \\
\hline
\end{tabular}

Totel 


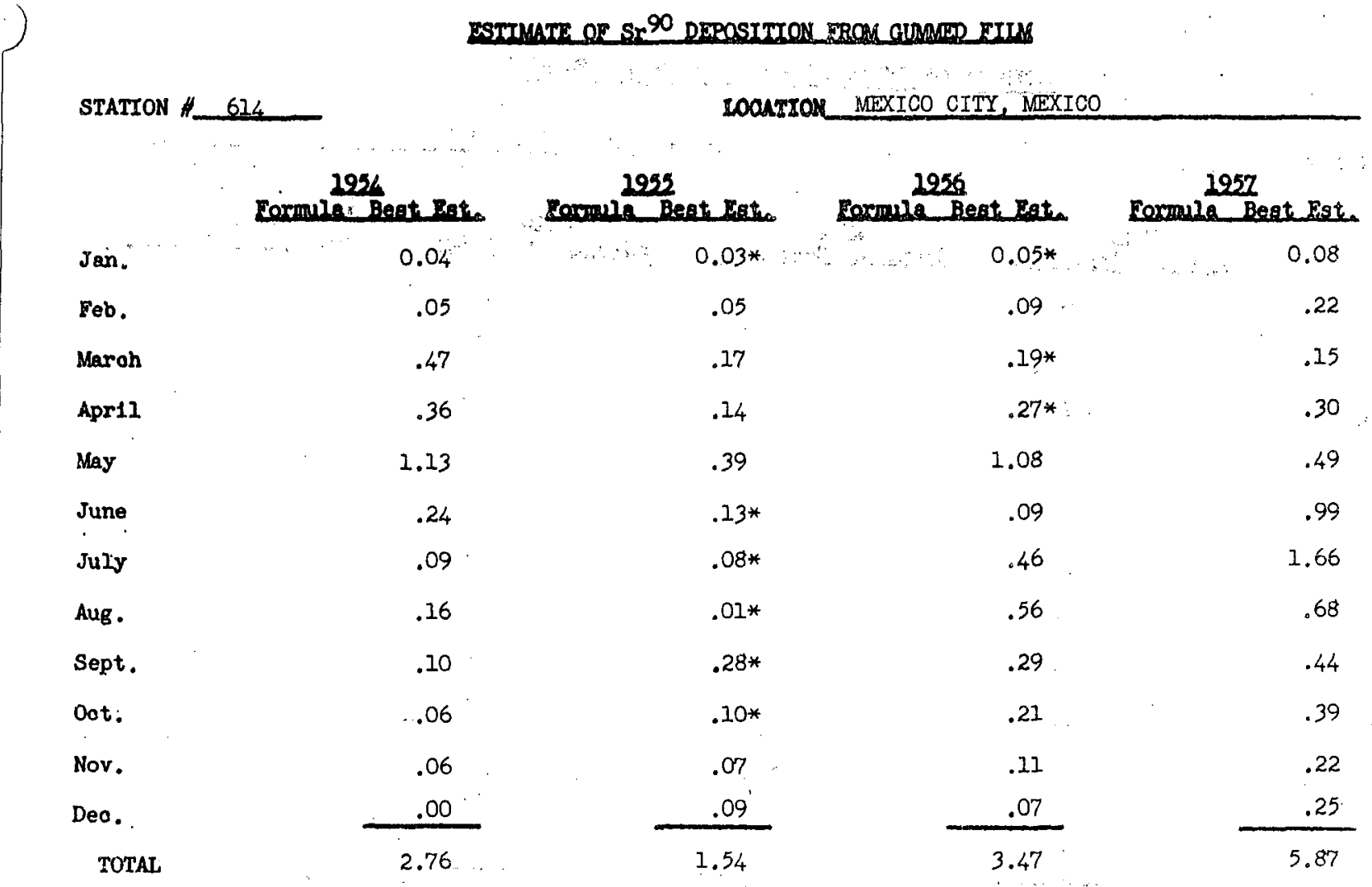

Formula Best Fot. Cormula Best set.

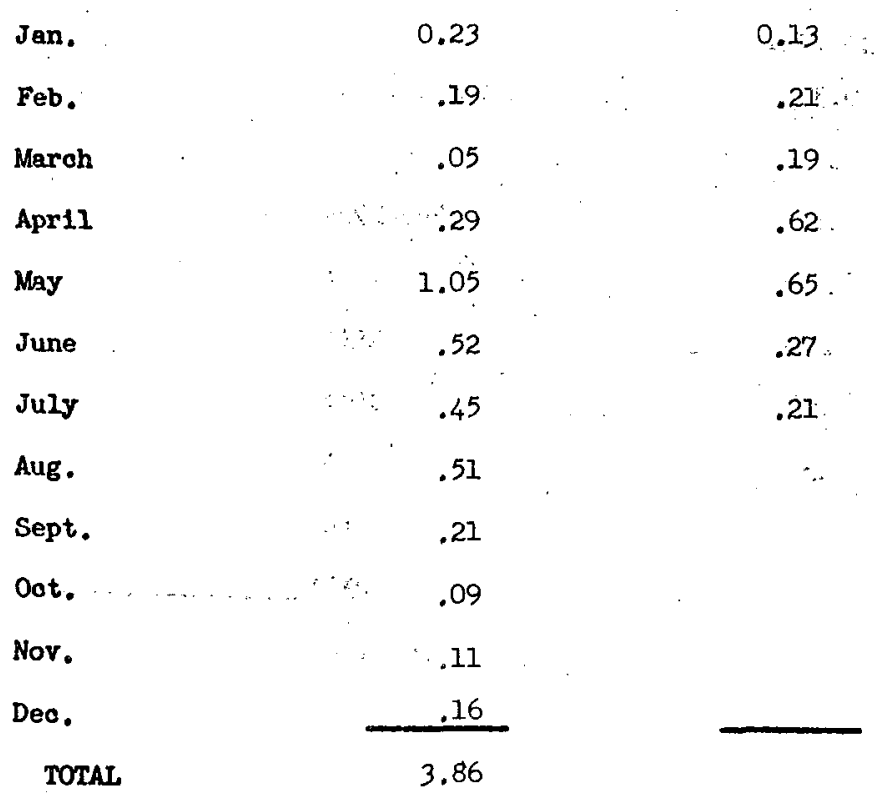

\begin{tabular}{rr} 
Pre-1954 & 0.92 \\
$\therefore 1954$ & 2.76 \\
1955 & 1.54 \\
1956 & 3.47 \\
1957 & 5.87 \\
1958 & 3.86 \\
1959 & \\
\hline
\end{tabular}

Total 


\section{ESTIMATE OF INFINITY $\gamma$ DOSE FHOM GUMMD FILM}

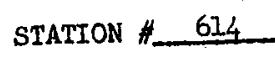

\section{LOCATION MEXICC CITY, MEXICO}

2954.2955 Eormula Best Eat.

0.0

Jan.

Feb.

March

April

May

June

JuIy

Aug.

Sept.

oct.

Nov.

Dec.

TOTAL
.1

2.6

1.9

6.5

.9

.2

.2

.1

.1

.1
12.7
Eecrula Best Eat.

$0.1 *$

.1

.3

.3

.7

$.2 *$

$.1 *$

$.0 *$

$.5 *$

$.2 *$

.1

.1

2.7
1956 Eacmula Best Est.

$0.1 *$

.1

$.3 *$

$.4^{*}$

1.6

.2

1.0

1.2

.6

.4

.2

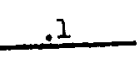

6.2
2957

Formula Best Est.

0.1

.4

.2

.6

.8

1.8

2.7

1.1

.8

.9

.4

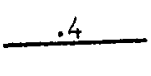

10.2
Jan.

Feb.

March

Apr11

May

June

July

Aug.

Sept.

oct.

Nov.

Dec.

TOTAL
0.4

.3

.1

.5

2.1

1.0

1.2

1.3

.5

.3

.3

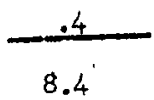

0.3

.4

.4

1.1

1.1

.5

.3
Pre-1954 $\quad 3.9$

$1954 \quad 12.7$

$1955 \quad 2.7$

$1956 \quad 6.2$

$1957 \quad 10.2$

$1958 \quad 8.4$

1959

Total 
STATTON \#-615

1254

Eormule Best rst.

Jan.

seb.

Maroh

April

May

June

July

Aug.

Sept.

oct.

Nov.

Dec.

TOTAL
0.04

.03

.14

.06

.05

.04

.02

.05

.07

$.01 * *$

.09

$.03 * *$

.63
1955

Foxmule Best Rst.

$0.03 *$

.08

$.05 *$

$.02 *$

$.10 *$

$.01 *$

$.02 *$

$.04 *$

$.43 *$

$.08=4$

.05

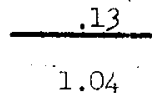

2956

Cormula Best Est.

$0.11 *$

.18

$.23 *$

$.09 * *$

.33

.16

.28

$.23 * *$

$.12 * *$

$.14 * *$

$.13 * *$

$.08 * *$

2.08
1957

Formula Best Est.

$0.05 * *$

$.06 * *$

$.06 * *$

$.05 * *$

.07

.12

.08

.14

.17

.07

.03

.41

1958

Formula Best Est.

Jan.

0.18

F́eb.

.17

March

.08

April

.02

May

.02

June

.06

July

.15

Aug.

.06

Sept.

.05

oct.

.05

Nov.

.03

Dec.

TOTAL

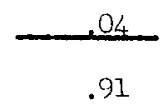

1259

Cormula Best Eat.

0.13

.08

.02

.09

.07

.03

$\therefore$

\begin{tabular}{rc} 
Pre-1954 & $\cdots$ \\
1954 & .63 \\
1955 & 1.04 \\
1956 & 2.08 \\
1957 & 1.31 \\
1958 & .91 \\
1959 & \\
\hline
\end{tabular}

Total 
STATION \# 615

IOGATION BOGOTA, COLOMBIA

1954 Formula Best Est.

Jars.

Feb.

March

April

May

June

JuIy

Ang.

Sept.

Oct.

Nov.

Deo.

TOT'AL
0.0

.1

.8

.3

.3

.2

.1

.1

.2

$.0 * *$

.2

$.1 * *$

2.4
1956 Formuli Best Est.

$0.2 *$

$0.1 *$

.2

$.1 *$

$.0 *$

$.2 *$

$.0 *$

$.0 *$

$.1 *$

$.7 *$

$.1 *$

.1

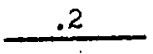

1.8
.3

$.3 *$

$.2 * *$

.5

.3

.6

$.5 * *$

$.3 * *$

$.3 * *$

. 3**

$.2 * *$

4.0
1957

Formula Best Est.

$.1 * *$

$.1 * *$

$.1 * *$

$.1 * *$

.1

.2

.1

.2

.3

.1

.1

.7

2.2

1958 Formula Best Est.

Jan.

0.3

1959

Eormula Best Eat.

Feb.

.3

March

.2

Apri1

.0

May

.0

June

.1

July

Aug.

Sept.

oct.

Nov.

.2

0.3

.2

.0

.2

.1

.0

\begin{tabular}{rr} 
Pre-1954 & -- \\
1954 & 2.4 \\
1955 & 1.8 \\
1956 & 4.0 \\
1957 & 2.2 \\
1958 & 1.9 \\
1959 & \\
\hline
\end{tabular}

Total

.1

Dec.

TOTAL

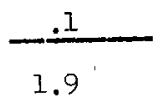

1.9 


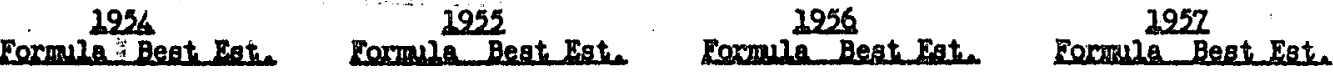

Jain.

Feb.

Maroh

Apr11

May

June

July

Aug.

Sept.

Oot:

Nov.

Deo.

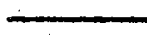

TOTAL

\begin{tabular}{lc} 
& \multicolumn{2}{c}{$\frac{1958}{29}$} \\
Jan. & \\
Féb. & 0.17 \\
Maroh & .03 \\
April & .02 \\
May. & .10 \\
June & .04 \\
July & .08 \\
Aug. & .16 \\
Sept. & .03 \\
Oct. & .04 \\
Nov. & .06 \\
Dec. & .05 \\
Tornt & -
\end{tabular}

\section{9}

Rormula Bert Eat.

0.06

.09

.06

.12

Pre-1954

1954

.00

2955

1956

1957

1958

1959

Total

.07

.07

.03

.03

TOTAL 
STATION \#-622

2954 Formula Best Est.

Jen.

Feb.

March

April

May

June

July

Aug .

Sept.

Det.

Nov.

Dec.

TOTAL

Corme thest Esta
LOCATYON BOLOTA COLOMBIA, TIBAITATA Eormula Best Est. Formula Best Est. Formula Beat Est. Eormula Best Est. Forrula Best Est. Formula Beat Est. Eormula Best Est. Formula Best Est. Formula Beat Est. 
ESTIMATE OF Sr 90 DEPOSTTION FROM GUMMED FILM

STATION \#. 701

LOCATION PRESTWICK, SCOTLAND

Eormula Best Est.

0.02

Jan.

Feb.

March

Apr11

May

June

July

Aug.

Sept.

Oot.

Nov.

Deo.

TOTAL
.13

.01

.01

.02

.04

.07

.17

.37

.. .26

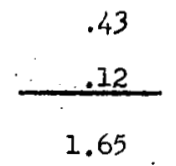

$\frac{1955}{\text { Formula Best Este }}$

$0.05 *$

$.03 *$

$.07 *$

$.24 *$

$.10 *$

$.24^{*}$

$.07 *$

$.10 *$

$.10 *$

$.15 *$

.37

.66

2.18
1956

Eormila Best Est.

$0.41 *$

.18

$.21 *$

$.24 *$

.72

.20

.58

.11

.48

.38

.35

.38

4.24
1957

Eormula Best Est.

0.30

.23

.59

.64

.46

.33

.30

.37

.54

.38

.29

19

4.61

1958

1959.

Formula Best Este Eormula Best Est.

Jan.

0.42

0.52

Feb.

.22

1.04

March

.47

April

.94

May

.49

.93

1.72

June

.49

JuIy

.23

Aug.

.19

Sept.

.17

oet.

.21

1.16

.89

.44

.16

.19

.19

\begin{tabular}{rr} 
Pre-1.954 & 1.05 \\
1954 & 1.65 \\
1955 & 2.18 \\
1956 & 4.24 \\
1957 & 4.61 \\
1958 & 4.84 \\
1959 & \\
\hline
\end{tabular}

Nov,

.22

.15

Dec.

TOTAL

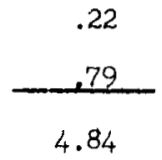

Total 
STAIION \# 701

LOCATION PRESTWICK, SCOTLAND

1954

$1955 \quad \underline{1956}$

Formila Best Est.

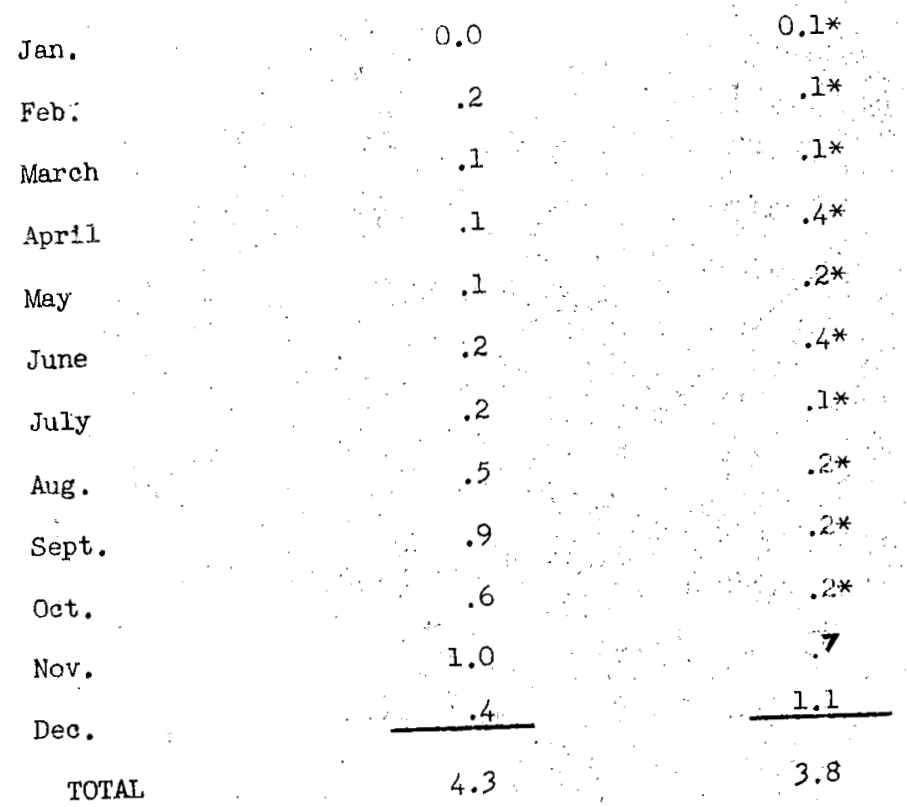

$0.7 *$

.3

$.3 *$

$.4^{*}$

1.1

.4

1.4

.2

1.0

.7

.7

.7

7.9
0.5

.4

9

1.2

.8

$\therefore 6$

.5

$\because 6$

.9

.8

$\therefore 6$

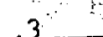

8.1 Formula Best Est.

1952

cormila Best Est.

Jan.

Feb.

March

April

May

June

July

Aug.

Sept.

oct.

Nov.

Dec .

TOTAL
0.7

.4

.9

1.6

1.0

1. 0

.6

.5

.4

.7

.8

$-1.9$

10.5
1.1

2.1

1.8

3.1

2.0

1.5

.7

.2

.3

$\therefore 3$

.2
Pre-1954 $\quad 1.8$

$1954 \quad 4.3$

$1955 \quad 3.8$

$1956 \quad 7.9$

$1957 \quad 8.1$

$1958 \quad 10.5$

1959

Total 
$195 \%$

1955

Eormula Beat Pat. Formul Beat Ests

Jain.

0.07

Feb.

Maroh

Apr11

May

June

July

Aug .

Sept.

Oot:

Nov.

Deo.

TOTAL
.07

.08

.01

.01

.04

.09

.12

.11

.10

.40

.28

1.38
$0.03 *$

.68

$.24 *$

$.21 *$

$.38 *$

.29*

$.15 *$

$.11 *$

$.33 *$

$.20 *$

.11

.36

3.09
1956

Eormula Best Dat.

$0.24 *$

.06

$.18 *$

$.56 *$

.59

.25

.31

.20

.16

.17

.12

.13

2.97
2957

Eormula Best Est.

0.15

.39

.88

.47

.27

.37

$.89 * *$

$.87 * *$

$2.85 * *$

$.09 * *$

$.06 * *$

.31

7.60

2958

Formula Best Egte. Cocmula Beat Eat.

Jan.

0.24

0.52

Feb.

.27

.41

March

.66

Apri1

.83

May.

1.16

June

July

.79

.29

Aug.

.34

Sept.

.15

Dot.

.32

1.35

1.51

.90

.90

.33

.37

.39

\begin{tabular}{rl} 
Pre-1954 & 0.58 \\
1954 & 1.38 \\
1955 & 3.09 \\
1956 & 2.97 \\
1957 & 7.60 \\
1958 & 5.85 \\
1959 & \\
\hline
\end{tabular}

Nov.

.18

Dec.

TOTAL

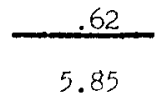


STATION \# 702

LOCATTON_BHEXN MAIN_ CERMANY

1956 $1955 \quad 1256 \cdots 1927$ Formule Best Egt. Formila Begt Este Formle Best Este Eormula Best Est.

Jan.

0.1

Feb.

March

Apr11

May

June

July

Aug .

Sept.

Det.

Nov.

Dec.

TOTAT.

\begin{tabular}{l}
.1 \\
.2 \\
.1 \\
.1 \\
.1 \\
.3 \\
.3 \\
.3 \\
.2 \\
.9 \\
.4 \\
\hline 3.1
\end{tabular}

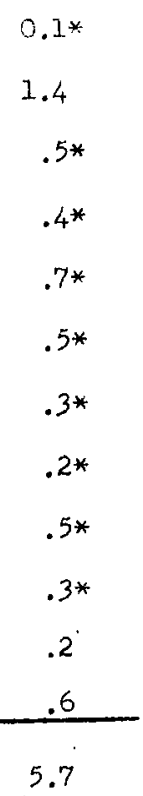

$0.4 *$

.1

$.3 *$

$.8 *$

1.0

.5

.7

.4

.3

.3

.2

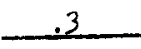

5.3

0.3

.6

1.4

.9

.5

.7

$1.5 * *$

$1.4 * *$

$3.6 * *$

$.2 * *$

$.1 * *$

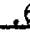

11.8

Formula Best Eata Cormula Best Est.

Jan.

0.4

1.1

Feb.

.4

March

1.3

.8

Apr11

1.4

2.6

2.7

May

2.3

1.6

1.5

1.5

June

.8

.5

July

.9

Aug.

.6

.6

\begin{tabular}{rr} 
Pre-1954 & 2.1 \\
1954 & 3.1 \\
1955 & 5.7 \\
1956 & 5.3 \\
1957 & 11.8 \\
1958 & 12.4 \\
1959 & \\
\hline
\end{tabular}

Sept.

.4

oct.

1.0

Totel

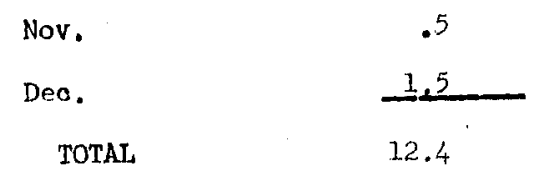


ESTTMATE OF $5 r^{90}$ DEPOSITTON RROM GLAMRD EIUK

STATION

LOATION PRETORIA, UNION OF SOUTH AFRICA

\begin{tabular}{|c|c|c|c|}
\hline Dormul 1954 Beat rat. & Bermuln Best Bstn & 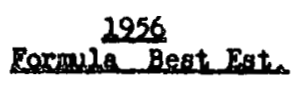 & Eermula Begt Est. \\
\hline 0.04 & $0.03 *$ & $0.03 *$ & 0.10 \\
\hline .03 & .06 & .04 & .03 \\
\hline .01 & $.03 *$ & $.02 *$ & .06 \\
\hline .01 & $.02 *$ & $.04 *$ & .05 \\
\hline .09 & $.19 *$ & .22 & .06 \\
\hline .03 & $.02 *$ & .04 & .21 \\
\hline .02 & $.02 *$ & .08 & .43 \\
\hline .09 & $.02 *$ & .03 & .11 \\
\hline .23 & $.03 *$ & .19 & .14 \\
\hline .21 & $.16 *$ & .25 & .13 \\
\hline .21 & .02 & .12 & .05 \\
\hline 01 & $.18^{\circ}$ & .09 & .08 \\
\hline .88 & 0.78 & 1.15 & 1.45 \\
\hline
\end{tabular}

Jain.

Feb.

Maroh

Apr11

May

June

July

Aug.

Sept.

Oot:

Nov.

Deo.

TOTAL

.88

0.78

1.45

1958

Formila Best Bat. Formula Best Bat.

Jan.

0.08

0.11

Feb.

.18

.06

March

.10

Apr11

May.

.04

$.14 * *$

June

.46

July

.07

July

.05

Aug.

.04

.16

Sept.

.22

.20

.22

.20

.19

oot.

.19

Nov.

.19

Dec.

TOTAL.

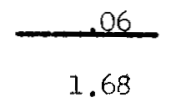

.18

.II

.07

.08

\begin{tabular}{rr} 
Pre-1954 & 0.68 \\
1954 & .88 \\
1955 & 0.78 \\
1956 & 1.15 \\
1957 & 1.45 \\
1958 & 1.68 \\
1959 & 1.72 \\
\hline Total & 8.3
\end{tabular}

1.72 
1254 Formula Best Esti: 0.0

\begin{tabular}{lc} 
Jan. & 0.0 \\
Feb. & .1 \\
March & .0 \\
AprtI & .1 \\
May & .4 \\
June & .1 \\
JuIy & .1 \\
Aug. & .1 \\
Sept. & .6 \\
Oct. & .5 \\
Nov. & .2 \\
Dec. & .0 \\
\hline TOTAI & 2.2 \\
\hline
\end{tabular}

Formula Best Est.

$0.1 *$

.I

$.1 *$

$.0 *$

$.3 *$

$.0 *$

$.0 *$

$.0 *$

$.0 *$

$.2 *$

.0

.3

1.1
1956 Eormula Best Est.

$0.0 *$

. I

$.0 *$

$.1 *$

.6

.1

.2

.1

.4

.5

.2

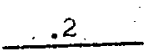

2.5
1252 Formula Best Est. 0.2 .1 $.1 \cdot \cdots$ .1 .1 .4 .7 .2 .2 .3 .1 .1 2.6
1958 Formila Best Est.

Jan.

Feb.

March

Aprit.

May

June

isuly

Nug.

Sept.

oct.

Nov.

Dec.

TOTAL.
0.1

.3

.2

.1

.9

.1

.1

.1

.5

.6

.6

$-1$

3.7
0.2

$$
.1
$$$$
.3 * *
$$

.3

.3

.4

.3

.3

.3

.2

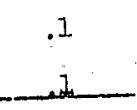

2.9 


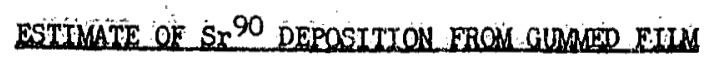

STATTON :

LOdATYON DHAIIRAN, SAUDI ARABIA

2954

1956

2257

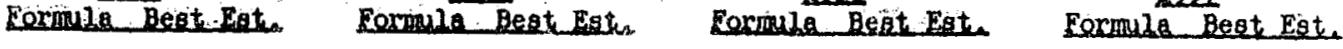

Jain。

0.04

$.05 *$

$0.20 *$

0.33

Feb.

.10

.05

.28

.40

March

.09

Apri1

.08

May

.07

June

.06

July

.06

Aug.

.04

Sept.

.09

oct.

.03

Nov.

.00

Deo.

.09

TOTAL

.75

$.02 *$

$.53 *$

.39

$.15 *$

$.26 *$

1.24

$.10 *$

.24

1.16

$.02 *$

.13

.22

$.01 *$

.12

.20

$.01 *$

.29

.30

$.00 *$

$.04 *$

.19

.25

$.01 *$

.05

.10

.08

.08

.15

$\frac{.18}{2.45}$

.19

.61

4.86

Jan.

Feb.

March

Apriz

May.

June

July

Aug.

Sept.

Det.

Nov.

Dec.

TOTAI.

\section{Formula Best Eat.}

1.07

.19

.42

.60

.36

.23

.18

.12

.06

.03

.23

39

3.88
2959

Rermila Best-Eit.

1.16

.59

1.37

.54

1.26 .

.32

.15 .

.28

.08

.10

$\begin{array}{rr}\text { Pre-1954 } & 0.93 \\ 1954 & .75 \\ 1955 & .61 \\ 1956 & 2.45 \\ 1957 & 4.86 \\ 1958 & 3.88\end{array}$

1959

Total

.12 
slintols 4705

LOCATION DHAHRAN, SAJDI ARABIA

1954

1956

1252 Formula Best Est Formule Best Est. Formula Best Este Formula Best Est.

$\begin{array}{lc}\text { Jas. } & 0.0 \\ \text { Fub. } & .8 \\ \text { Merch } & .5 \\ \text { Aprit } & .4 \\ \text { May } & .4 \\ \text { Mune } & .2 \\ \text { Hity } & .2 \\ \text { Aug. } & .1 \\ \text { Sept. } & .2 \\ \text { Det. } & .1 \\ \text { Nov. } & .0 \\ \text { Dec. } & .2 \\ \text { notal } & 2.5\end{array}$

\begin{tabular}{l}
$0.1 *$ \\
.1 \\
$.3 *$ \\
$.3 *$ \\
$.2 *$ \\
$.0 *$ \\
$.0 *$ \\
$.0 *$ \\
$.0 *$ \\
$.0 *$ \\
$.0 *$ \\
.2 \\
\hline 1.2 \\
\hline
\end{tabular}

$0.3 *$

.4

$.8 *$

$.4 *$

.4

.3

.3

.4

.4

.1

.2

.3

4.3
0.6

.7

.6

2.3

2.0

.4

.3

.5

.4

.2

.2

.3

8.5

Foymla Best ist. Formula Best Est.

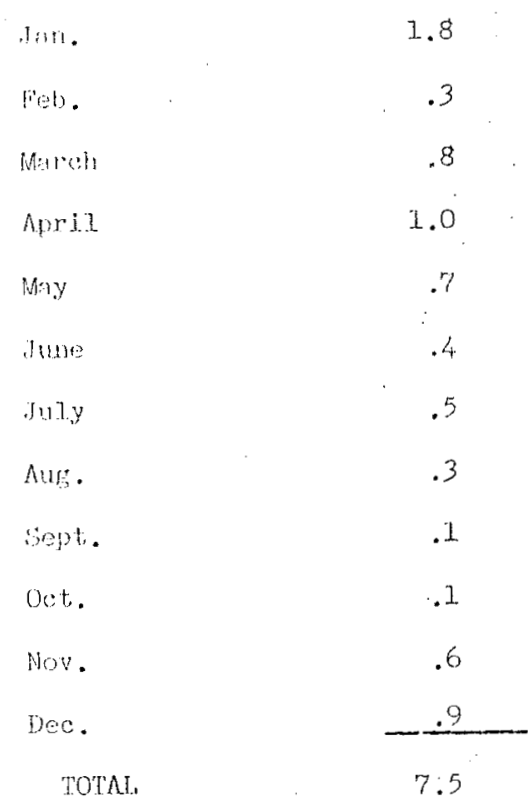

2.5

I.2

2.6

1.0

2.2

.5

.2

.4

.1

.1

\begin{tabular}{rr} 
Pre-1.954 & 2.5 \\
1954 & 2.5 \\
1955 & 1.2 \\
1956 & 4.3 \\
1957 & 8.5 \\
1958 & 7.5 \\
1959 & \\
\hline
\end{tabular}

Tota1 


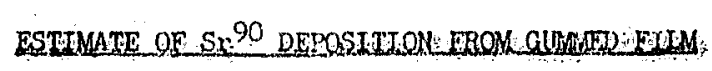

ETATSON H. 706

TOOATIOHL SIDI SLIMANE, MOROCCO

$125 \%$

$\cdot$

Jain.

0.04

Cosmula Beat Est.

$0.06 *$

2956

1257.

Fint.

.04

.15

waichi

.07

Aprest

.03

Wey

.03

none?

.04

.143

.02

Aug.

.02

Sept.

.03

ont.

.06

Nov.

.19

nee.

Tornt
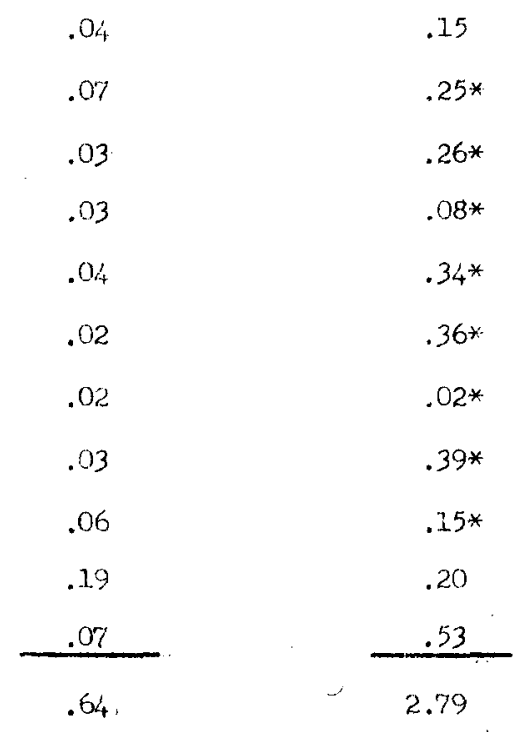

Eormula Beste Fat.

Cocmula Best Est.

$0.48 *$

0.26

.87

.15

$.86 *$

.51

$.99 * \quad .57$

.93

.35

.25

.13

.09

.32

.24

.28

.11

.59

.27

.53

.22

.26

.13.

.65

5.44

4.60

1958.

Fornuda Best Eate Formula Bestukst.

\begin{tabular}{|c|c|}
\hline Jani, & 0.36 \\
\hline Febs. & .30 \\
\hline Maroh & .42 \\
\hline Ar:i1 & 4.27 \\
\hline May. & .75 \\
\hline Mune & .72 \\
\hline July & .18 \\
\hline Aur. & $.10^{* * *}$ \\
\hline Sept. & $.33 * *$ \\
\hline bet. & $.53 * *$ \\
\hline Nov. & .86 \\
\hline Dec. & 2.13 \\
\hline IOTAL. & 10.95 \\
\hline
\end{tabular}

0.62

1.01

1.59

1.02

1.51

.15

.38

.17

.12

\begin{tabular}{rc} 
Pre-1954. & $0.79 * *$ \\
1954 & .64 \\
1955 & 2.79 \\
1956 & 5.44 \\
1957 & 4.60 \\
1958 & 10.95 \\
1959 & \\
\hline
\end{tabular}

Total 


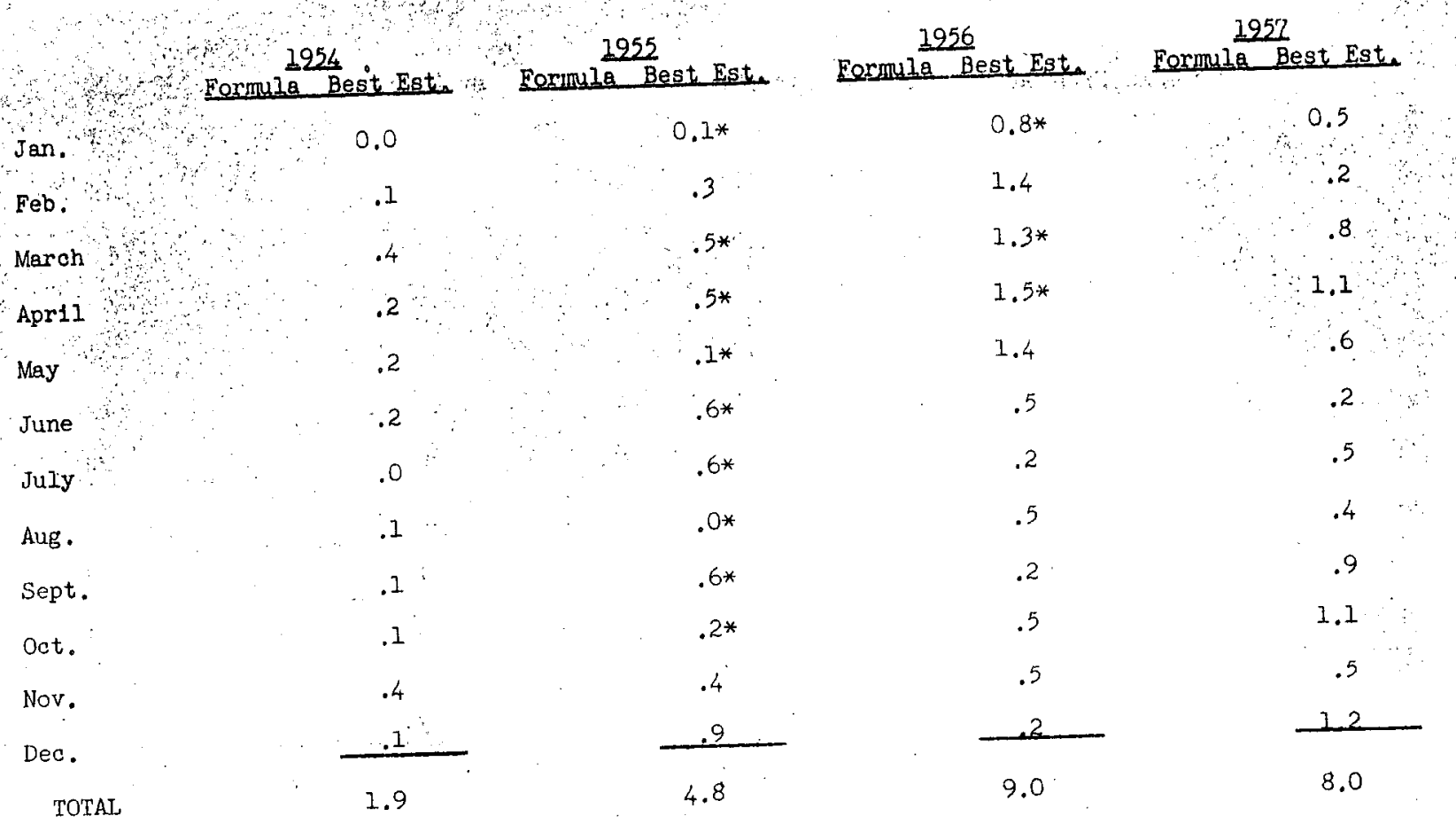

\begin{tabular}{|c|c|c|c|c|}
\hline & Formula Best Est. & Formula Best Est. & & \\
\hline Jan. & 0.6 & 1.3 & & \\
\hline Feb. & .5 & 2.0 & & \\
\hline March & .8 & 3.0 & Pre-1954 & $2.0 * *$ \\
\hline April & 7.2 & 1.9 & 1954 & 1.9 \\
\hline May & 1.5 & 2.7 & 1955 & 4.8 \\
\hline June & 1.4 & .3 & 1956 & 9.0 \\
\hline July & .5 & .6 & 1957 & 8.0 \\
\hline Aug. & $.3 * *$ & .3 & 1958 & 23.4 \\
\hline Sept. & $.8 * *$ & .2 & 1959. & L \\
\hline oct. & $2.3 * *$ & & Total & \\
\hline Nov. & 2.4 & & & \\
\hline Dec. & 5.1 & - & & \\
\hline TOTAL. & 23.4 & & & \\
\hline
\end{tabular}




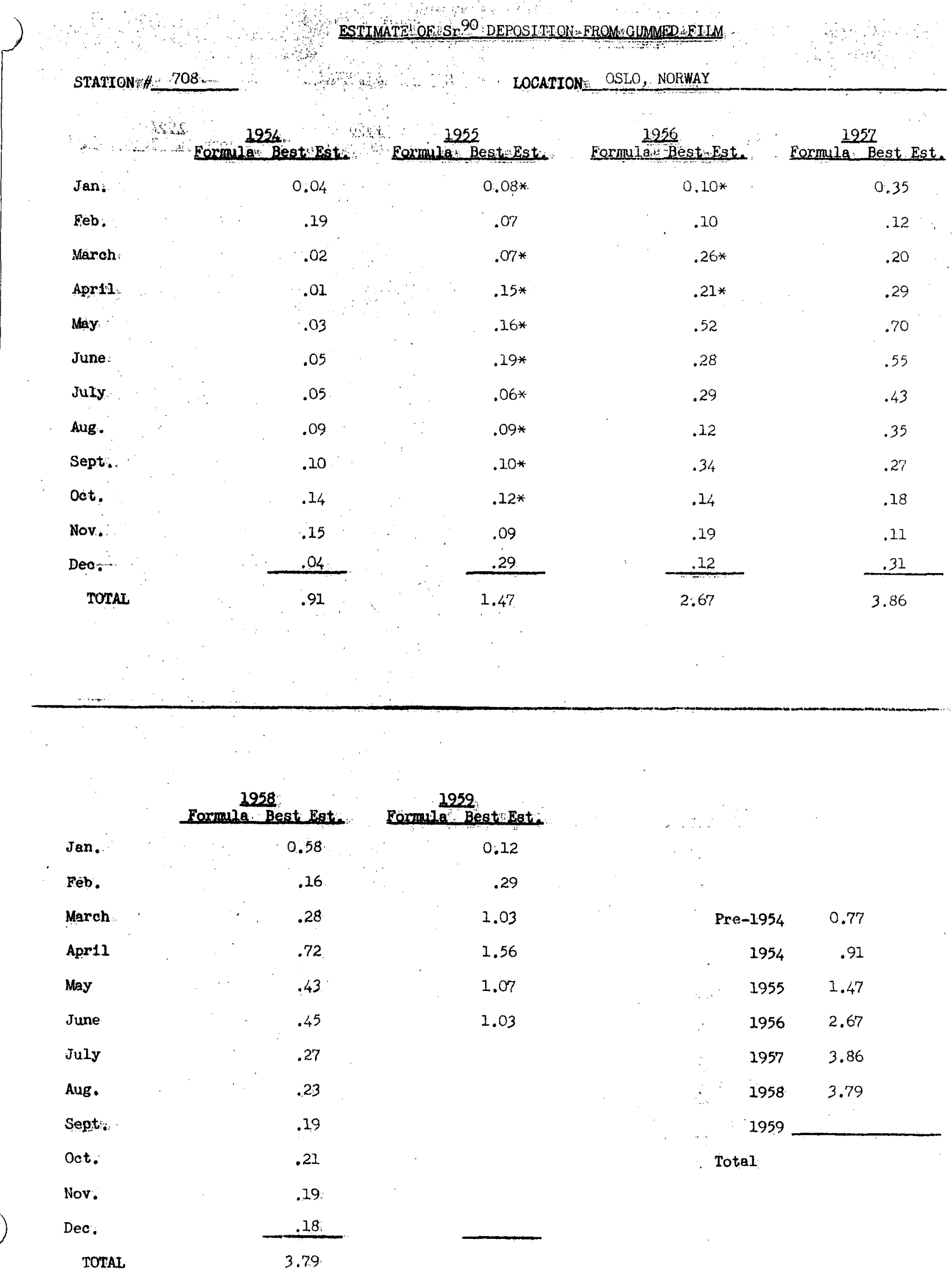


STATION \#. 708

LOCATION_ OSLO, NORWAY

1955

$\frac{1254}{\text { Best Est. }}$

Jan.

Feb.

March

Apri1

May

June

July

Aug.

Sept.

oct.

Nov.

Dec.

TOTAL

$$
.3
$$

\section{Formule Best E}

.1

.1

.1

.2

.2

.2

.3

.3

.3

.1

2.2

\section{Forrula Best Este.}

$0.2 *$

.1

$.1 *$

. 3*

. 3*

. 3*

$.1 *$

$.1 *$

$.2 *$

$.2 *$

.2

.5

2.6

\section{Formula Best Est. \\ $0.2 *$}

.2

$.4 *$

$.3 *$

.8

.5

.7

.3

.7

.3

.4

.2

5.0
1952

Eormula Begt Est.

0.6

.2

.3

.6

1.2

1.0

.7

.5

.5

.4

.2

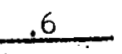

6.8

Formula Best Est.

1959

1.0

Formila Best Est.

Jan.

.3

Feb.

.5

March

1.2 .

Apr 11.

May

June

.9

July

.9

Aug.

.7

Sept.

.3

Oct.

.4

Nov.

.6

0.3

Dec.

TOTAL

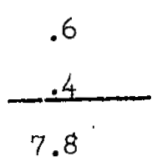

2.0

2.8

1.9

1.7

$\begin{array}{rr}\text { Pre-1954 } & 1.9 \\ 1954 & 2.2 \\ 1955 & 2.6 \\ 1956 & 5.0 \\ 1957 & 6.8 \\ 1958 & 7.8\end{array}$

1959

Total 
STATON 710

LOQRIOX TRIPOLI, LIBYA, WHEELUS AFB

$\begin{array}{rrr}1956 & 2953 & 1957\end{array}$

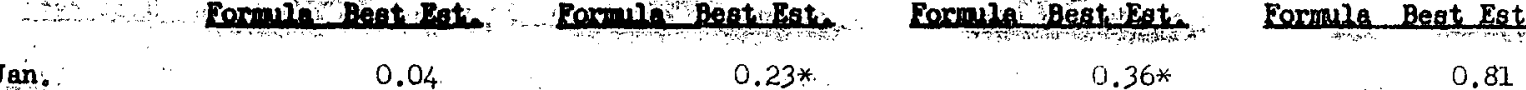

Feb $\quad .11$

March:

Apr:11:

May .

June

JuIy,

Aug.

Sept.

Oot:

Nov.

Deo.

TOTAL
.06

.11

.11

.18

.07

.03

.11

.24

.42

30

1.78

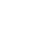
.

\author{
$\therefore .09 * *$
}

$.17 *$

$.22 *$

$.31 *$

$.27 *$

$.10 *$

$.01 *$

$.08 *$

$.42 *$

.33

.80

3.03
$9.36 *$

.77

$.61 *$

$2.53 *$

.46

.13

.05

.08

.3I

.33

.41

.37

6.41
6.92

.17

.31

.63

.72

1.12

.20

.28

.76

1.15

.38

.39

\section{. 2958}

Formula Best Dat.

Jan.

Féb.

March

Apri1

May.

June

July

Aug.

Sept.

Oct.

Nov.

Dec.

TOTAL
1.50

.57

.36

.84

.42

.62

.18

.10

.33

.53

$.35 * *$

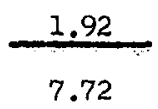

2959

Bormula Peat Bat,

2.76

3.20

1.92

1.23

Pre-1954

0.80

1954

1.78

3.81

.74

.73

.16

.27

1955

3.03

1956

6.41

1957

6.92

1958

7.72

1959

Total 
STATION \#_ 710

1254 Formula Best Est.

Jan.

0.0

Feb.

March

Apr11

May

June

JuIy

Aug.

Sept.

Det.

Nov.

Dec.

TOTAI.
.2

.4

.6

.6

.7

.2

.1

.3

.6

1.0

.5

5.2
LOCATION_TRIPOLI, LIBYA, WHEELUS AFB

1956 Formula Best Est.

$$
0.6 *
$$$$
1.2
$$$$
.9 *
$$$$
3.8 *
$$$$
.7
$$$$
.3
$$$$
.1
$$$$
.2
$$$$
.6
$$

.6

.8

.6

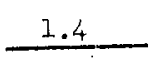

5.5

10.5
1957

Formula Best Est.

1.4

.3

.5

1.1

1.2

2.0

.3

.4

1.2

2.6

.8

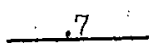

12.5
Jan.

Feb.

March

April

May

June

July

Aug.

Sept.

oct.

Nov.

Dec.

TOTAI.
2.5

1.1

.7

1.4

.8

1.2

.5

.3

.8

2.3

1. $0 * *$

4.6

17.2
5.9

6.4

3.7

2.2

6.6

1.2

1.2

.3

.4

\begin{tabular}{rr} 
Pre-1954 & 3.0 \\
1954 & 5.2 \\
1955 & 5.5 \\
1956 & 10.5 \\
1957 & 12.5 \\
1958 & 17.2 \\
1959 & \\
\hline
\end{tabular}

Tota1 

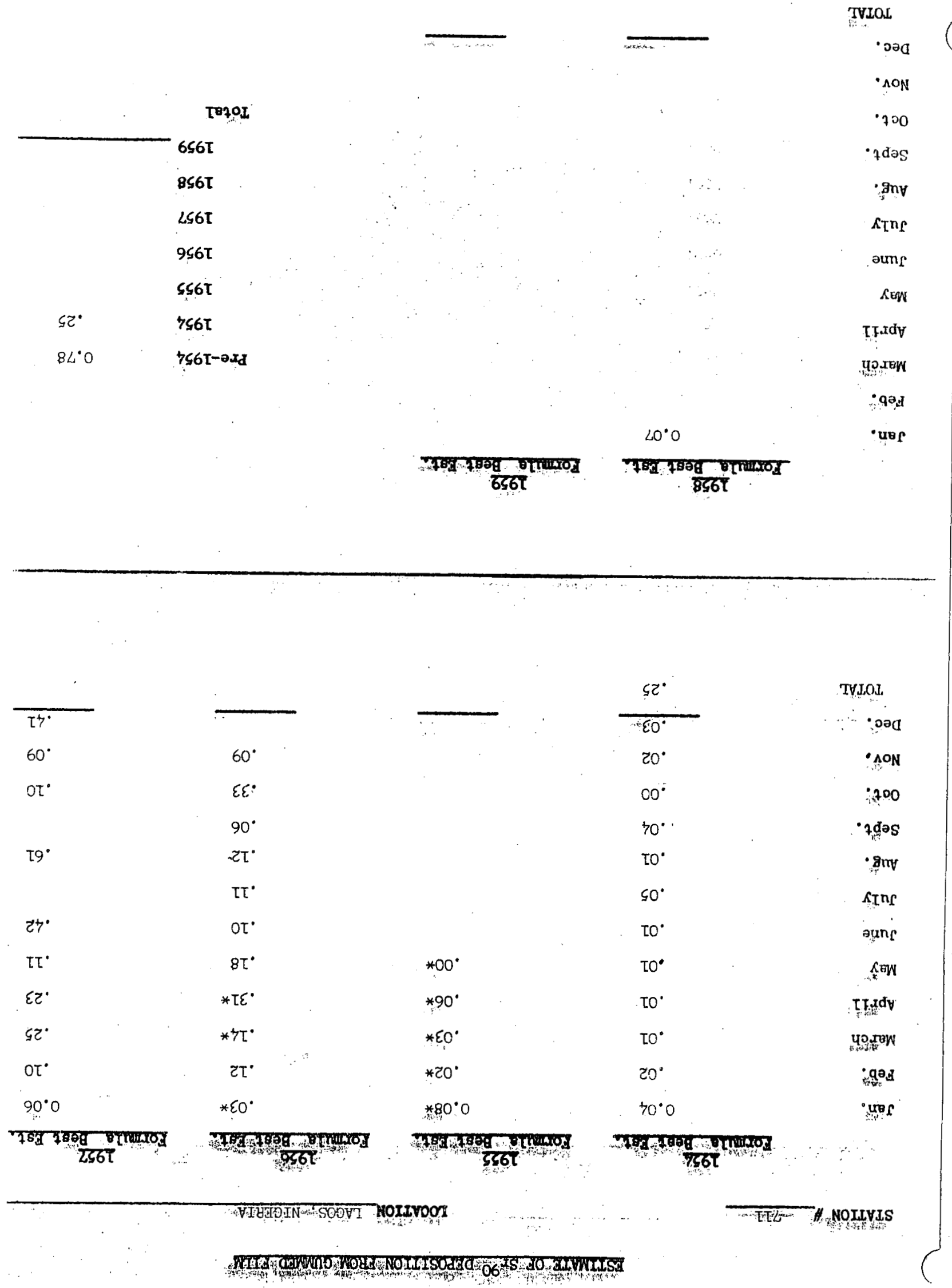
$1954 \quad 1955$

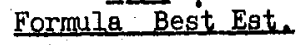

0.0

Jan.

Feb.

March

April

May

June

July

Aug.

Sept.

oct.

Nov.

Deo.

TOTAL
.0

.0

.1

.1

.0

.1

.0

.1

.0

.0

.0

.4
1956

Formula Best Est.

$0.2 *$

$.0 *$

$.1 *$

$.1 *$

$.0 *$

Eormule Best Est.

$0.0 *$

.2

$.2 *$

$.5 *$

.3

.2

.2

.3

1.0

.1

.6

.2
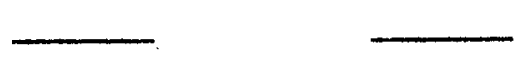

Formula Best Est.

0.1

.2

.4

.4

.2

.7

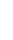

.2

.2

.7

\section{Formula Best Est. Formula Best Est.}

Jan.

0.1

Feb.

March

Apri1

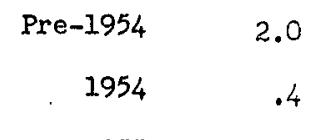

May

1955

June

1956

July

1957

Aug.

1958

Sept.

1959

Oct.

Total

Nov.

Dec.

TOTAL. 
STATION \# 713

LOOATION DUREAN, UNTON OF SOU'SH AFRICA

\begin{tabular}{|c|c|c|c|c|}
\hline & Eormula Beat Yato & Cormula Beet Rst. & Eacsula Best Eat. & $\frac{2957}{\text { Eormula Be日t Est. }}$ \\
\hline Jan. & 0.04 & $0.03 * *$ & $0.03 *$ & 0.07 \\
\hline Feb. & .02 & $.06 * *$ & .05 & .05 \\
\hline March & .01 & $.03 * *$ & .02 & .06 \\
\hline ApriI & .02 & $.02 * *$ & $.04 * *$ & .05 \\
\hline May & .02 & $.19 * *$ & $1.51 * *$ & .06 \\
\hline June & .01 & $.01 *$ & $.04 * *$ & .04 \\
\hline July & .03 & $.02 * *$ & .09 & .13 \\
\hline Aug. & .02 & $.02 * *$ & .08 & .20 \\
\hline Sept. & .04 & $.26 *$ & .05 & .20 \\
\hline Oot. & .09 & $.06 *$ & .06 & .21 \\
\hline Nov, & .10 & .06 & .14 & .08 \\
\hline Dec. & .05 & .04 & .07 & .07 \\
\hline TOTAL, & .45 & .80 & 2.18 & 1.22 \\
\hline
\end{tabular}

Cormula Best Est. Bormula Best. EBt.

Jan.

0.08

Feb.

March

April

May

June

July

Aug.

Sept.

oct.

Nov.

Dec.

TOTAL
.22

.01

.01

.10

.03

.07

.05

.18

.10

.09

.09

1.03
.10

.12

.07

.72

.04

.04

.13

.10

$.0 \%$

.22

.09

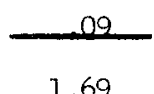

1.69

\begin{tabular}{rc} 
Pre-1954 & $0.79 * *$ \\
1954 & .45 \\
1955 & .80 \\
1956 & 2.18 \\
1957 & 1.22 \\
1958 & 1.03 \\
1.959 & 1.69 \\
\hline Total & 8.2
\end{tabular}

Note: Pretoria only station available for interpolation 
1254 - 1955 Formula Best Est.

Formula Best Est.

$0.1 * * *$

$.1 * *$

Feb.

March

Apr11

May

June

July

Aug.

Sept.

oet.

Nov.

Dec.

TOTAL
0.0

.0

.1

.1

.1

.0

.1

.1

.2

.2

.2

.1

1.1
$.1 * *$

$.0 * *$

$.3 * *$

$.0 *$

$.0 * *$

$.0 * *$

$.4 *$

$.1 *$

.1

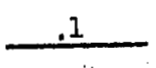

1.3
2956 Eormuia Best Est.

$0.0 *$

.1

$.0 *$

$.1 * *$

$3.6 * *$

$.1 * *$

.2

.2

.1

.1

.3

.1

4.9
1957 - Cormula Best Est.

0.1

.1

.1

.1

.1

$\because 1$

.2

.4

.3

.5

.2

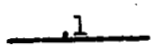

2.3

1952

Formula Best Est.

Formila Best Eat.

Jan.

0.1

0.2

Feb.

.5

March

.0

$$
.2
$$

.1

Apr 11

.0

May

June

July

.2

.1

.2

Aug.

.1

Sept.

.4

oct.

.3

Nov.

.3

Dec.

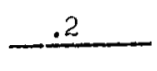

TO'TAI,

2.4

1.3

.1

.1

.2

.2

.1

.2

.1

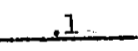

2.9

\begin{tabular}{rl} 
Pre-1954 & $2.0 * *$ \\
1954 & 1.1 \\
1955 & 1.3 \\
1956 & 4.9 \\
1957 & 2.3 \\
1958 & 2.4 \\
1959 & 2.9 \\
\hline Tota1 & 17
\end{tabular}




\section{ESTTMATE OF SI 90 DEPOSITION FROM GUMAED FILM}

STATYON

LOCATYON MONROVIA. IIBERIA

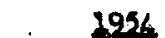

1955

1256

1952

Dormula Beat. Det.

Jan.

0.04

Feb.

Maroh

April

May

June

Juty

Aus.

Sept.

oot.

Nov.

Deo.

TOTAL
Eormuze Best Est.

$0.05 *$

$.02 *$

$.05 *$

$.12 *$

$.10 *$

$.09 *$

$.06 *$

$.07 *$

$.07 *$

.19*

.09

.05

.96
Eormuia Best Est.

$0.08 *$

.06

$.13 *$

$.16 *$

.30

.29

.22

.40

.29

.19

.17

.16

2.45
Formule Best Est.

0.11

.10

.13

.07

.13

.35

.77

.50

.32

.13

.09

.09

2.79

1958 1959

Formule Best Date Eormula Best Eat.

Jen.

0.60

0.27

Feb.

.19

.45

March

.19

.41

April

.30

.33

May

.33

June

.29

July

.30

.31

.38

Aug.

.32

Sept.

.24

oct.

.21

.14

Nov.

.09

Dec.

.15

TOTAL

3.21

\begin{tabular}{rr} 
Pre-1954 & $0.79 * *$ \\
1954 & 1.38 \\
1955 & .96 \\
1956 & 2.45 \\
1957 & 2.79 \\
1958 & 3.21 \\
1959 & \\
\hline
\end{tabular}

Total 
STATION \# $711_{4}$

LOCATION MONROVIA, LIBERIA

1254 Formule Best Est.

$\begin{array}{lc}\text { Jan. } & 0.0 \\ \text { Feb. } & .1 \\ \text { Merch } & .5 \\ \text { April } & .4 \\ \text { May } & .5 \\ \text { June } & 1.0 \\ \text { July } & .6 \\ \text { Aug. } & .4 \\ \text { Sept. } & .2 \\ \text { Oct. } & .3 \\ \text { Nov. } & .3 \\ \text { Dec. } & .2 \\ \text { ToTAL } & 4.5\end{array}$

1952 Eorinule Best. Est.

$0.1 *$

$.0 *$

$.1 *$

$.2 *$

$.2 *$

$.2 *$

. . $*$

.].*

$.1 *$

$.3 *$

.1

.1

1.6
1956

Eormula Best Est.

$0.1 *$

$.2 *$

$.2 *$

$.2 *$

.5

$: 6$

.5

.9

.7

.4

.3

.3

4.8
1927

Formula Best Est:

0.2

.2

.2

$: 1^{1}$

.2

.6

1.3

.8

.5

.3

.2

.2.

4.8 .

1958 Formula Best Est.

1959

Formula Best Est.

Jan.

Feb.

March

Apr 11

May

June

JuIy

Aug.

Sept.

oct.

Nov.

Dec.

TOTAI.
1.0

.3

.4

.5

.7

.6

.8

.8

.5

.6

.3

$-4$

6.9
0.6

.9

.8

.6

.5

.6

.2

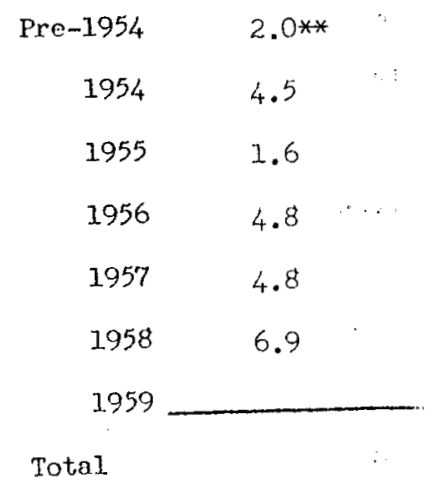


STATION

LOOATION: MLAN, ITAIY.

2934

1953.

2956

1957.

Dormula Degt Pst.

Jen:

Peis:

0.07

Maroh

Apri1

May:

June

Juty.

Aug.

Sept.

Oot.

Nov.

Dec.

TOTAL
Borule Beat Bati.

$0.02 *$

$.04 *$

$.05 *$

$.07 *$

$.34 *$

$.73 *$

$.30 *$

$.16 *$

$.10 *$

$.48 *$

.06

.14

2.49
Bormula Bent. Fat.

$0.21 *$

.06

$.39 *$

$.76 *$

.98

.39

.29

.20

.55

.17

.03

.06

4.09
Eermula Beet Est.

0.08

.09

.10

.19

.13

.25

.21

.18

.19

.09

.04

.05

1.60

2958

Formula Best Bgt. Formula Beat Eat.

Jan.

0.17

0.27

Feb:

.29

March

.23

.34

April

.17

May

.19

June

.19

July

.08

Aug.

.10

Sept.

.08

oct.

.08

Nov.

.07

Deo.

TOTAL

.15

1.80

.74

1.54

1.40

.96

.74

20

ep.

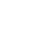

\begin{tabular}{rc} 
Pre-1954 & $0.79 * *$ \\
1954 & .79 \\
1955 & 2.49 \\
1956 & 4.09 \\
1957 & 1.60 \\
1958 & 1.80 \\
1959 & \\
\hline
\end{tabular}

Total 
ESTTMATE OF INFINITY $y$ DOSE FROM GUMMED FILM

STATION \#_...715

LOCATION MIIAN, ITALY

$1954 \quad 2955$ Formula Best Est. Eormule Best Est. $\frac{1956}{\text { Eormula Best Est. }}$

Formula Best Est.

Jan.

0.1

$0.0 *$

$0.3 *$

0.1

\section{Feb.}

March

Apr11

May

.1

.2

$.1 *$

$.1 *$

.2

June

.2

July

Aug.

Sept.

oct.

Nov.

Dec.

TOTAL

.1

.2

.2

.0

.2

.0

.9

3.7

$.1 *$

$.6 *$

$.7 *$

$.5 *$

$.3 *$

$.2 *$

$.8 *$

.1

.2

.1

$.6 *$

$1.1 *$

1.6

.7

.6

.4

1.2

.3

.1

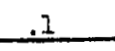

7.1

.1

.2

.3

.2

.4

.3

.3

.3

.2

.1

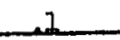

2.6

\begin{tabular}{|c|c|c|}
\hline & Formula Best Est. & Formula Best Est. \\
\hline Jan. & 0.3 & 0.6 \\
\hline Feb. & .5 & .7 \\
\hline March & .5 & 1.4 \\
\hline Aprell & .3 & 2.8 \\
\hline May & .4 & 2.4 \\
\hline June & .4 & 1.6 \\
\hline suly & .2 & 1.2 \\
\hline Aug. & .3 & .3 \\
\hline Sept. & .2 & \\
\hline oot. & .2 & \\
\hline Nov. & .2 & \\
\hline Des. & .4 & 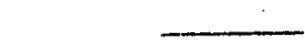 \\
\hline TOTAI, & 3.9 & \\
\hline
\end{tabular}

\begin{tabular}{rl} 
Pre-1954 & $2.0 * *$ \\
1954 & 1.9 \\
1955 & 3.7 \\
1956 & 7.1 \\
1957 & 2.6 \\
1958 & 3.9 \\
1959 & \\
\hline
\end{tabular}

Total 
Cormi 1954. Best Dat.

Jan.

Feb.

March

Apri1.

May

June

JuIy

Aug.

Sept.

oot.

Nov.

Deo.

TOTAL

$$
0.04
$$

.04

.10

.09

.15

.33

.21

.30

.26

.09

.03

.01

1.65
1955

Formula Best Est. $0.08 *$

.03

$.05 *$

$.10 *$

$.06 *$

$.22 *$

$.24 *$

$.31 *$

$.09 *$

$.06 *$

.01

.12

1.37
2956

Formula Best Est.

$0.05 *$

.09

$.11 *$

$.34 *$

.16

.14

.21

.27

.35

.22

.01

$\frac{.04}{1.99}$

1957

Formula Best Est.

0.05

.21

.12

.17

.36

.55

.57

.55

.40

.36

.04

.07

3.45

1958

Formula Best Eat.

0.19

Eormula Best Est.

Jen.

Feb.

March

April

May

June

July

Aug.

Sept.

Oct.

Nov.

Dec.

TOTAL
.06

.11

.17

.16

.51

.70

.53

.43

.10

.02

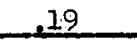

3.17

.

02

19
0.17

.26

.45

.74

$-\cdots$

.46

.16

.18 
1254 Formula Best Est.

$\begin{array}{lc}\text { Jen. } & 0.0 \\ \text { Feb. } & .1 \\ \text { March } & .5 \\ \text { April } & .5 \\ \text { May } & .7 \\ \text { June } & 1.3 \\ \text { July } & .7 \\ \text { Aug. } & .8 \\ \text { Sept. } & .7 \\ \text { Oct. } & .2 \\ \text { Nov: } & .0 \\ \text { Dec. } & 5.6 \\ \text { TOTAL } & .1\end{array}$

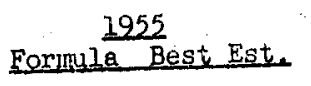

$0.2 *$

.1

$.1 *$

$.2 *$

$.1 *$

$.4 *$

$.4 *$

$.5 *$

$.1 *$

$.1 *$

.0

.2

2.4
1956 Formula Best Est.

$0.1 *$

.2

$.2 *$

$.5 *$

.3

.3

.5

.6

.8

.4

.0

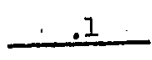

4.0
1952

Formula Best Est.

0.1.

.3

$.2 \%$

.3

.6

1.0

.9

.9

.7

.8

.1

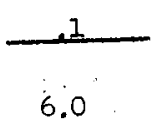

1958

1959

Formula Best Est.

Jan.

0.3

Formula Best Est.

Feb.

.1

March

.2

Apri1

.3

May

.3

June

1.0

July

1.9

Aug.

1.4

0.4

Sept.

1.0

Det.

.3

Nov.

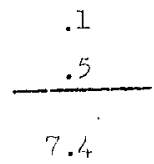

Dec.

TOTAL

.9

1.4

- -

.8

.3

.3

$\begin{array}{rr}\text { Pre-1954 } & 2.0 * * \\ 1954 & 5.6 \\ 1955 & 2.4 \\ 1956 & 4.0 \\ 1957 & 6.0 \\ 1958 & 7.4\end{array}$

1959

Total 


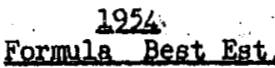

1255

Formul Best Est. Formula Best Est Formula mBest Esti Formula Best Est.

Jan.

Ver.

March

April

May

June

.52

July

.91

Aug .

1.29

Sept.

2.11

oot.

.09

Nov.

.20

Deo.

.09

TOTAL

1959

Formula Best Est. Formula Best Est

Jan.

0.17

0.32

Feb.

.39

March

.60

.69

Apri1

.79

May

.51

June

.84

July

.33

Aug .

.30

Sept.

.09

.71

Pre-1954

2.44

1954

1.93

1955

1.84

1956

.88

1957

oct.

.17

.45

.25 .

1958

4.90

1959

10.04

Nor.

.13

Dec.

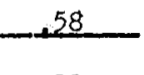

.29

Total

TOTAL

4.90

.16

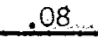

10.04 
STATION \# 722 LOCATYON VIENNA, AUSTRIA, WIEN HOHE WARTE

1254.

Formula Best Est. $1955^{\circ}$

Formula Best Est.
1956

Eormula Best Est.
1957

Eormula Best Est.

Jan.

F'eb.

March

Apri1

May

June

July

Aug .

Sept.

Oct.

Nov.

Dec.

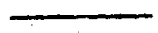

$\therefore 9$

1.5

2.0

3.4

.2

.4.

1958

1952

Formula Best Este Formla Best Est.

Jan.

0.3

0.7

Feb.

.7

March

1.2

1.4

1.0

1.4

Apri1

May

1.6

.9

July

Aug.

.8

sept.

oct.

.2

.6

Nov.

.4

Dec.

1.4

TOTAT,

10.5

1.3

4.4

Pre-1954

1954

3.4

1955

3.1

1956

1.4

1957

$1958 \quad 10.5$

.7

1959

17.5

Total

.4

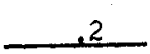

.4

.2

.1

17.5 
STATION \# 723 LOCATIONDURBAN, UNION OF SOUTH AFRICA, NATAL AIRPORT

1254

Eormula. Best. Est.

1955

Formula. Best Est.
1256

Formila Best Est
1957

Formule Best Est.

Jan.

Feb.

March

Apr11

May

June

.06

July

Aug .

.29

Sept.

Oot.

Nov.

.08

Dec.
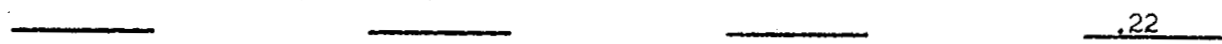

TOTAL

Formula Best Est. Eormula Begt Est.

Jan.

0.11

0.122

Feb.

.22

.08

March

.47

Apr11

.01

May

.18

June

.03

July

.05

Aug.

.19

Sept.

.19

Oct.

.06

Nov.

.03

Dec.

TOTAL

.07

1.61

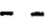

.09

.18

.10

.21

.15

.10

.1.1

.07

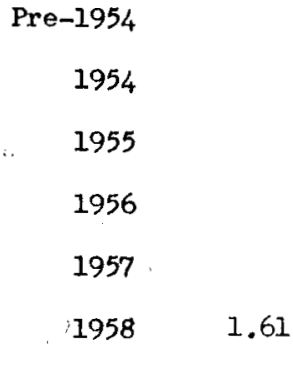

1959

Total 
STATION \# 723

LOCATION DURBAN, UNION OF SOUTH AFRICA, NATAL AIRPORT
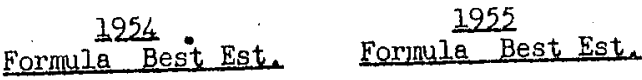

Jan。

Feb.

March

Apr11

May

June

July

Aug .

Sept.

Oct.

Nov.

Dec.

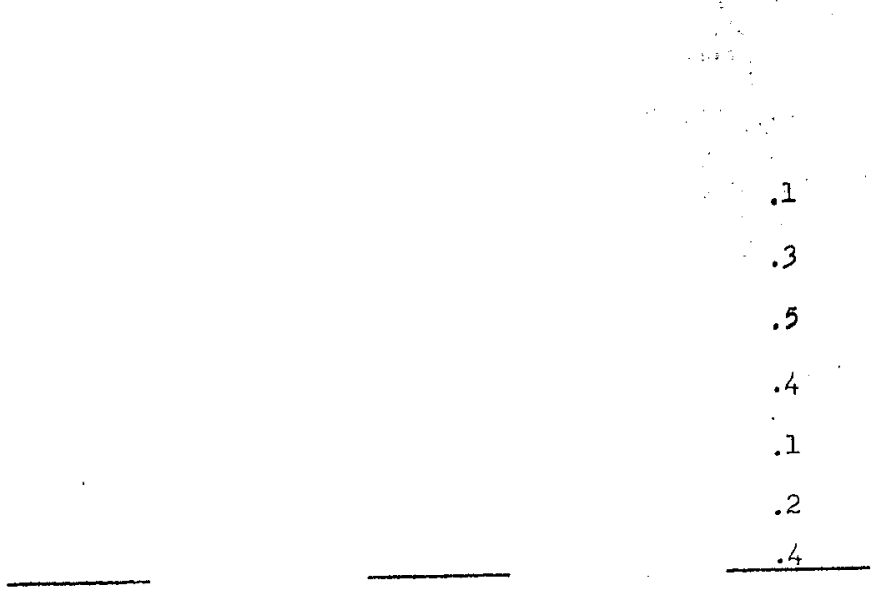

1958

Formula Best Est.

Jan.

Feb.

March

Aprit

May

June

fuly

Aug.

Sept.

oct.

Nov.

Dee.

TOTNL
0.2

.4

.9

.0

.4

.1

.1

.5

.4

.2

.1

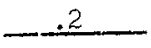

3.5
1959

Formula Best Est.

0.3

.2

$-$

.2

.3

.2

.3

.2

.1

.2

Pre-1954

1954

1955

1956

1957

$1958 \quad 3.5$

1959

Total 
STATION \#_ 724

LOCATION SALZBURG, AUSTRIA

Formula Best Est. Formula Best Est. Formula Best Est. Formula Best Est.

Jan.

Feb.

March

April

May

June

.70

JuIy

1.01

Aug .

.98

Sept.

2.85

Oct.

.07

Nov.

.10

Dec.

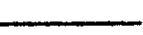

.42

TOTAL

1958

Jan.

Feb.

March

April

May

June

JuIy

Aug.

Sept.

oct.

Nov.

Dec.

TOTAL
0.26 .

.49

.31

2.28

1.22

1.20

.53

.34

.20

1.04

.22

$-\frac{.98}{9.07}$

1259

Formula Best Eat.

$\therefore 0.32$

.61

.90

1.66

Pre-1954

1954

3.08

1955

1956

1957

1958. 9.07

1959

10.70

Total

$=.15$

.12

.14

10.70 
STATION \#-72

LOCATION SALZBURG, AUSTRIA

\section{4}

1952

1956

Formula Best Est, Formula Best Est. Formula Best Est.

Eormula Best Est.

jan.

Feb.

March

April

May

June

July

Aug .

Sept.

oet.

Nov.

Dec.

TOTAL

1958.

Formale Best.Est.

Jan.

0.4

1959

Formula Best Est.

Feb.

.8

March

.6

April

4.0

May

2.5

June

2.2

July

1.5

Aug.

.9

Sept.

.5

oct.

3.6

Nov.

Dec.

TOTAL

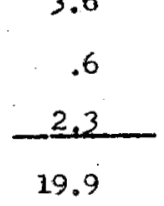

0.7

1.2

1.7

3.0

1.2

1.6

1.5

4.6

.2

.2

.8

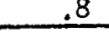


STATION \#-725

LOCATION INNSBRUCK; AUSTRIA

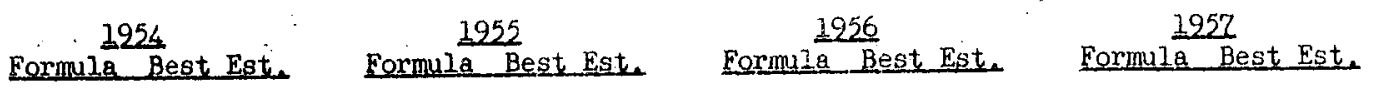

Jan.

Feb.

March

Apr11

May

June

1.02

JuIy.

.81

Aug.

2.40

Sept.

2.92

Oct.

Nov.

Dec.

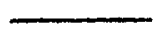

.43

TOTAL

1958

Formula Best Eat. Formula Best Est.

Jan.

0.22

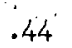

Feb.

.53

.53

March

.58

.53

April

1.22

1.48

Pre-1954

May

.66

2.15

1954

June .

.69

July

.37

Aug.

.43

Sept.

.23

oct.

.52

2.03

1955

Nov.

.09

Dec.

.89

TOTAL

6.43

.90

.43

.17

1956

1957

$1958 \quad 6.43$

1959

10.54

Total

$$
1.34
$$

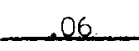

10.54 
STALION \#-725

\section{$1254 \quad 1955$}

Formula Besit Este Formula Best Est.

2956

1957

Jan.

Feb.

March

Apr11

May

June

1.8

July

1.3

Aug.

3.8

Sept.

4.7

oct.

.2

Nov.

.1

Dec.

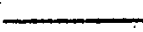

$\longrightarrow$

.8

TOTAL

Formula Best Este Formula Best Est.

Jan.

0.4

0.9

Feb.

.9

1.1

March

1.1

1.0

Apri1

2.1

May

1.3

June

1.3

July

1.0

Aug.

1.1

Sept.

.5

Oct.

1.8

2.7

3.7

3.4

1.5

.7

.3

.7

Pre-1954

1954

1955

1956

1957

1958

13.9

1959

18.1

Nov.

.3

2.0

Dec.

2.1

TOTAL,

1.3 .9

.1

18.1 
STATION \#+-726

LOCATION_KRIPPENSTEIN, AUSTRIA

1954

1957

Formula Best Est. Formula Bestist Formula Best Est. Formula Best Est.

Jan:

Feb.

March

April

May

June

July

Aug.

.81

Sept.

2.42

oct.

.13

Nov.

.04

Deo.

TOTAL
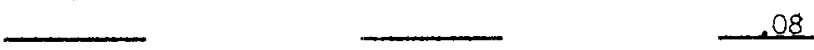
STATION \#-726

LOCATION KRIPPENSTEIN, AUSTRIA

Formule Best Est. Formula Best Est. Formula Best Est. $\quad \frac{1955}{1954}$ Formula Best Est.

Jan.

Feb.

Merch

Apr11

May

June

JuIy

Aug.

Sept.

oct.

Nov:

Deo.

TOTAL

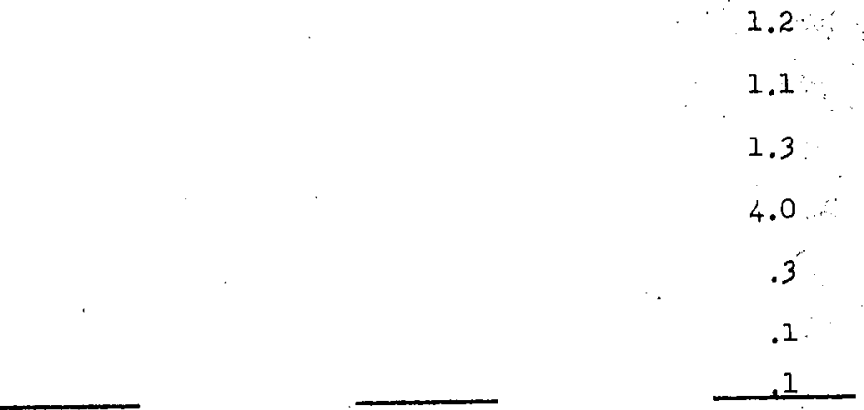

1958

Formula Best Eat.

0.1

.2

Feb.

March

Apri1

May

June

July .

Aug.

Sept.

Oct.

Nov.

Dec.

TOTAL
.9

.7

1.2

1.1

.7

.8

.4

.4

.4

$-\frac{.7}{7.6}$
1959

Formula Best Est.

0.4

.5

1.3

1.0

2.5

2.6

1.9

1.0

.5

.1

.5

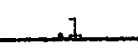

12.4
Pre-1954

1954

1955

1956

1957

$1958 \quad 7.6$

1959

12.4

Tota1 
STATION \#727

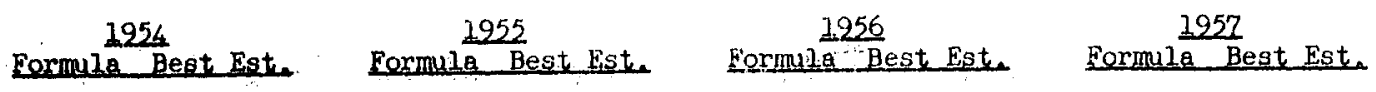

Jan.

Feb.

March

Apr11

May

June

1.06

July

1.08

Aug .

Sept.

Dot.

Nov.

Deo.

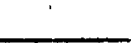

09

TOTAL

1959

Formula Best Est. Formule Best Est.

Jan.

0.18

0.14

Feb.

.07

.18

March

-. .24

.77

Apri1

1.22

1.38

Pre-1954

May

.65

1.55

1954

June

1.02

1.32

1955

July

.39

Aug.

.37

Sept.

.13

oct.

.08

Nov.

.20

Dec.

TOTAL

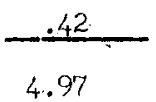

. .73

1.17

.30

.13

1956

1957

$1958 \quad 4.97$

1959

7.79

Total.

.02

.10

7.79 
STATION \# 727

Jan.

Feb.

March

Apr11

May

June

JuIy

Aug.

Sept.

oct.

Nov,

Dec.

TOTAL

1958 Formula Best Est.

Jan.

Feb.

March

Apri1

May

June

July

Nug.

Sept.

oct.

Nov.

Dec.

TOTAI,
0.3

.1

.5

2.1

1.3

1.9

1.0

1.0

.3

.3

.6

$-1.0$

10.4
1252

Formula Best Est.

0.3

.4

1.4

2.5

2.6

2.2

1.2

1.9

.5

.2

.0
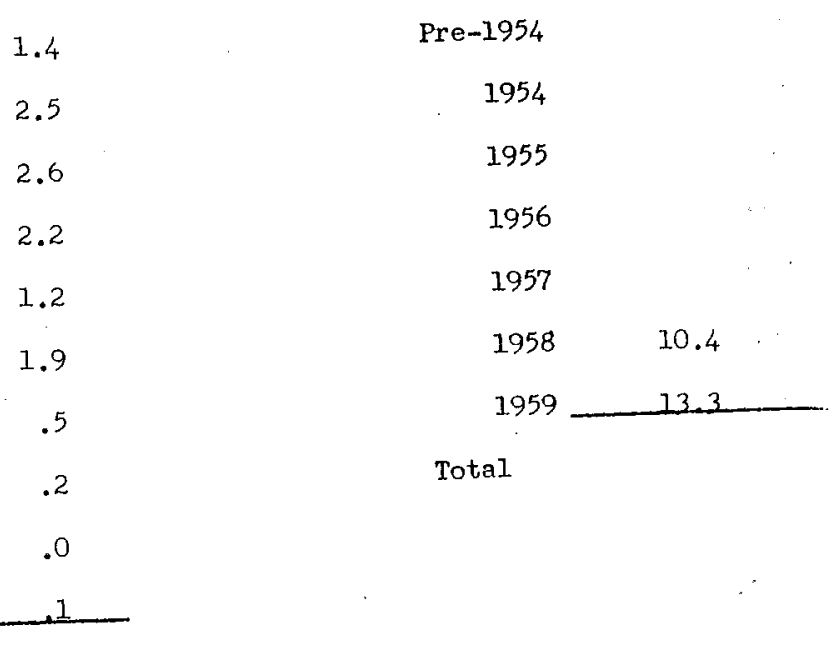

13.3 
ESTIMATE OF SY 90 DEPOSITION FROM GUMMED EIIM

STATTON \#

LOCATION PORT ELIZABETH, UNION OF SOUTH AFRICA

Eormule Best Est. Eormula Best Est. Formula Best Est. Formula Best Est.

Jan.

Feb.

Maroh

Apri1

May

June

.07

JuIy

Aug.

.16

Sept.

.15

Oat.

.13

Nov.

Deo.

TOTAI

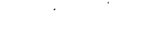

.05

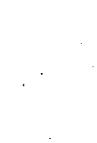

\begin{tabular}{|c|c|c|}
\hline & Formule Best Est. & Formi i Best Est. \\
\hline Jan. & 0.27 & 0.09 \\
\hline Feb. & .10 & .03 \\
\hline March & .36 & $.14 * *$ \\
\hline April & .05 & .14 \\
\hline May & .05 & .48 \\
\hline June & .06 & $.22 * *$ \\
\hline July & .04 & .06 \\
\hline Aug. & .02 & .09 \\
\hline Sept. & .08 & .06 \\
\hline Oct. & .06 & .12 \\
\hline Nov. & .07 & .06 \\
\hline Dec. & .10 & .10 \\
\hline TOTAL & 1.26 & 1.59 \\
\hline
\end{tabular}


STATION \#- $\because 28$

\section{$1254 \quad 1955$}

Formila Best Esti Formula Best Est. " Formula Best Esta

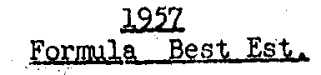

Tan.

Fer.

March

Apri1

May

June

July

.1

Aug.

.3

Sept.

Det.

Nov.

Dec.
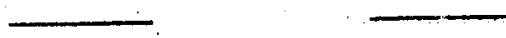

TO'TAL

Jan.

Ifeb.

March

April

May

June

July

nug.

Sret.-

oct.

Nov.

Dec.

TOIAT

\section{5}

.2

.7

.1

.1

.1

.1

.0

.2

.2

.2

.2

2.6
0.2

.1

$.3 * *$

.3

.8

$.4 * *$

.1

.1.

.1

.2

Pre-1954

1954

1955

1956

1957

$1958 \quad 2.6$

1959

Total 
STATION 729 LOCATIOR CAPETOWN, UNION OF SOUTH AFRICA

1954

Formula Beat Bet.

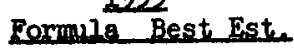

1256

2957

Jan.

Feb.

March

April

May

June

.18

JuIy

.11

Aug .

1.30

Sept.

oct.

Nov.

Deo.

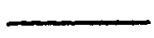

.10

TOTAS.

1958

Formula Best Est. Formula Best Est.

Jan.

0.14

0.07

Feb.

.16

March

.34

Apr11

.05

May

.05

June

.02

July

.09

Aug.

.09

Sept.

.02

Oct.

.03

Nov.

.41

.04

$.14^{* *}$

.08

Pre-1954

1954

.09

1955

.10

1956

:11

1957

.35

1958

1.64

1959

1.36

.13

:11

Total

Dec.

.24

.08

TOTAL

1.64

$\frac{.06}{1.36}$


STATION \# 729

IOCATION CAPETOWN, UNION OF SOUHH AFRICA

1254 Formula Best Eat.

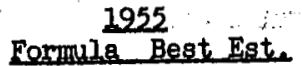

$\frac{1956}{19}$ Formla Best Esta
1952

Formula Begt Est.

Jan.

Feb.

Maroh

Apr11

May

June

JuIy

Aug .

Sept.

oct.

Nor:

Deo.

TOTAL

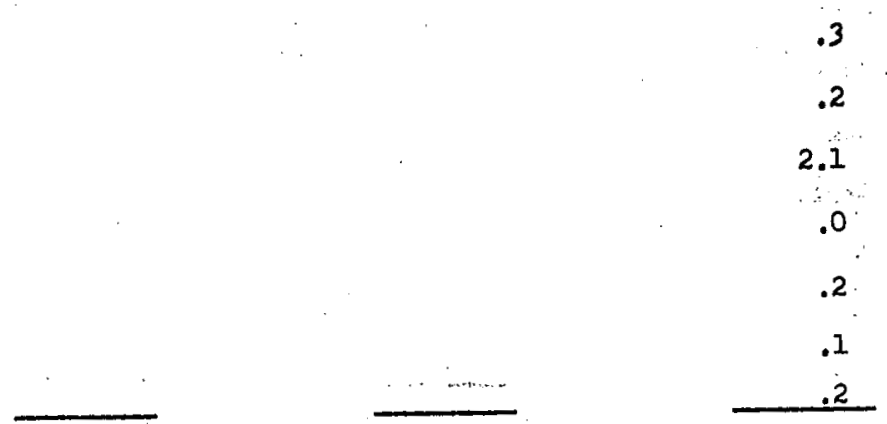

.3

(n)

.2

.1

.2

$1958 \quad 1959$

Formula Best Est. Formula Best Est.

Jan.

0.2

0.2

Feb.

.3

.1

March

.7

$.3 * *$

April

.1

May

.1

June

. 0

July

Aug.

Sept.

oct.

.1

Nov.

1.1

Dec.

TOTAL

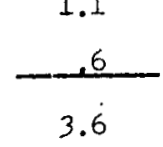

.1

.2

.2

.2

.6

.2

.2

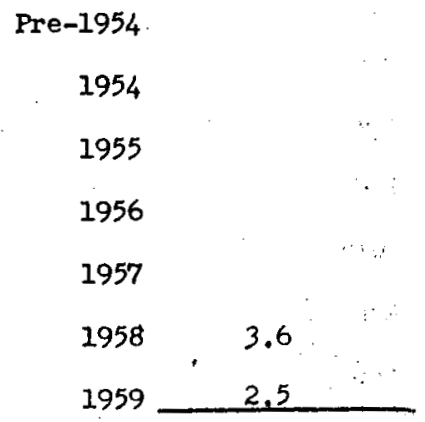

Tote1

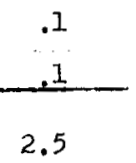


STATroN 730

LOCATTON BEAUFORT, UNION OF SOUTH AFRICA

Formulio Best.Est. Formula Best Esti. Formula Best Esta Formule Best Est.

Jan.

Fèb.

Märoh

Apprt1

May

June

.04

July

.16

Aug.

.14

Sept.

.02

oct.

.25

Nov.

.03

Dec.
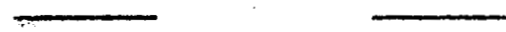

.08

TOTAL

2958

Farmula Best Est.

Jan.

1.24

Féb.

.10

.24

Márch

Apr11.

May

.08

.04

June

.09

July

.05

Aug.

.01

Sèpt.

.07

.29

Formula Best Est.

Oct.

Nov.

Dec.

TOTAL

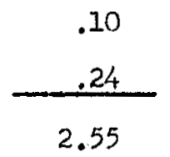

0.14

.39

$.14 * *$

.09

.19

.19

.19

.15

.19

.14

.08

.07

1.96
Pre-1954

1954

1955

1956

1957

$1958 \quad 2.55$

$1959+1.96$

Total 
STATION \#- 730

Formula Best Eat.

Jan。

Feb.

Maroh

Apr11

May

June

July

Aug.

Sept.

Oct.

Nov:

Deo.

TOTAL

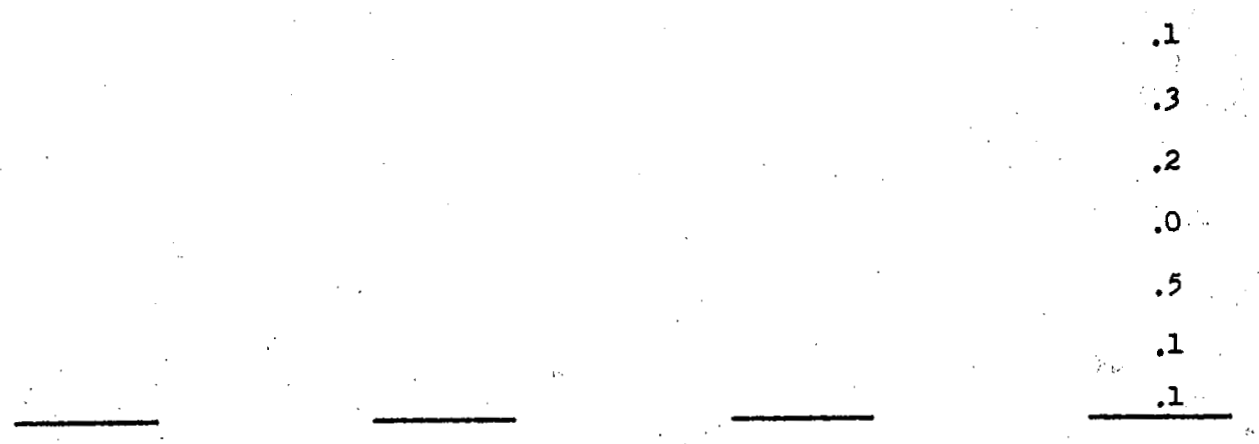

1958

Formula Best Est.

Jan.

Feb.

March

Apri1

May

June

July

Aug.

Sept.

oct.

Nov.

Dec.

TOTAL
2.0

. .2

.5

.1

.1

.2

.2

.0

.2

.8

.3

.6

5.1
1959

Formula Best Est.

0.3

.8

$.3 * *$

.2

.3

.3

.3

.2

.3

.2

.1

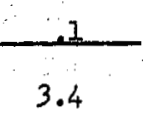

Pre-1954

1954

1955

1956

1957

$1958 \quad 5.1$

1959

3.4 


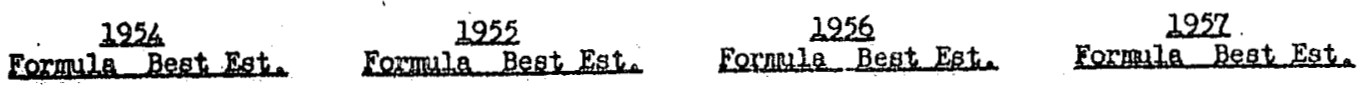

Jan.

Dob.

Maroh

Apr11

May

June

JuIy

Aug.

Sept.

Oot.

Nov.

Deo.

TOTAL

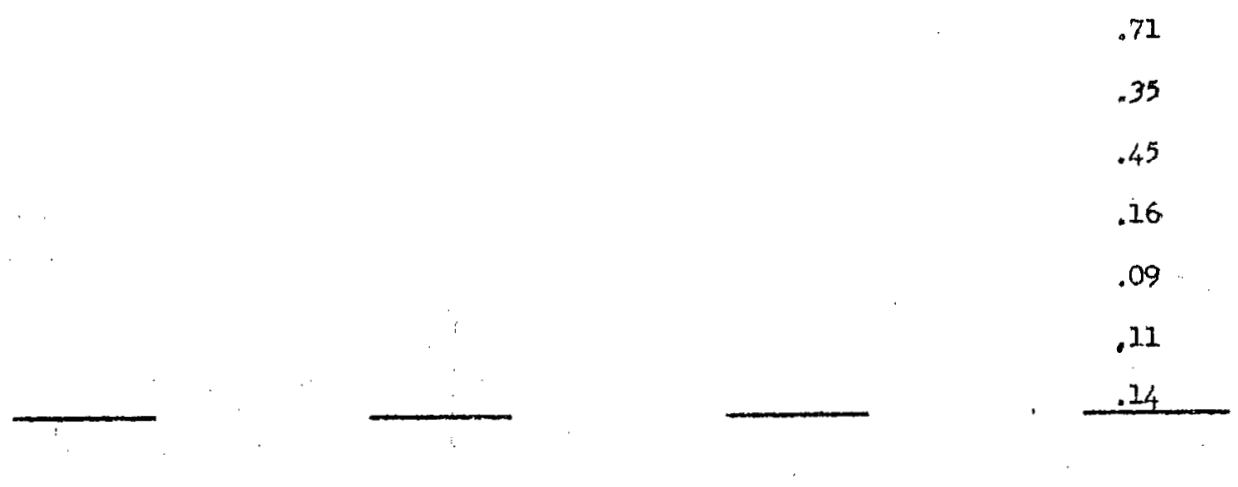

2958

Formula Beat Est. Formila Begt Est.

Jan.

0.15

0.05

Feb.

.13

.04

March

.11

$.14 * *$

April

.32

.26

Pre-1954

May

.12

.13

1954

1955

June

.12

.37

1956

Ju1y

.05

.23

1957

Aug.

.04

.06

1958

.17

1959

1.50

Sept.

.07

.14

Total

oct.

.13

.10

Nov.

.12

.08

Dec.

TOTAL

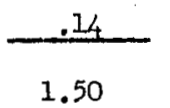

$\frac{.08}{1.77}$



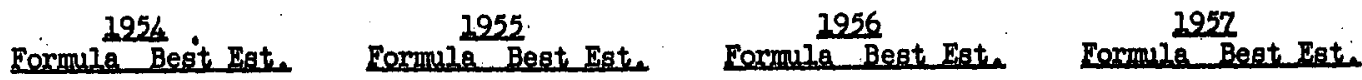

Jan.

Feb.

March

Apri1

May

June

July

Aug .

Sept.

1.2

Oet.

Nov.

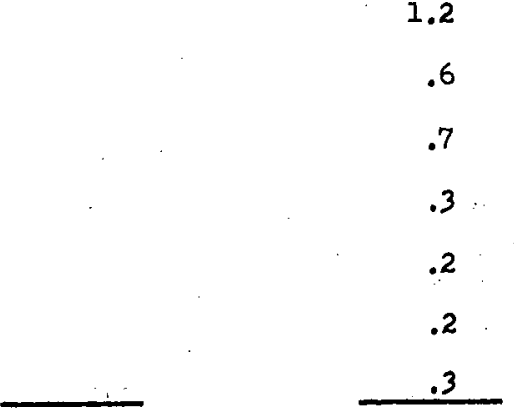

Deo.

TOTAL

1958

Formula Best Est.

1959

Eommla Best Est.

Jan.

0.3

.1

Feb.

.2

.1

March

.2

Apr11

.6

May

.3

June

.2

July

.1

Aug.

.1

Sept.

.2

.3**

.5

Pre-1954

.2

1954

1955

.6

1956

.4

1957

.1

1958

1959

3.4

.3

3.1

Oct.

.5

.2

Total

Nov.

.4

.2

Dec.

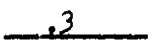

TOTAL

3.4

.1

3.1 
STATIONHA - 732

LOCATION WINDHOEK, UNION OF SOUTH AFRICA

2954.

Eormula Best, Sat.

Jan.

Feb.

March

April

May

June

Jüy

Aug .

Sept.

Oot.

Nov.

Deo.

TOTAL .

\section{5}

Eormula "Best Est.
1956

Formule Best Est.
1957

Formila Best Est. 


\section{4}

Formula Best, Eat.

1955

Eormu Best Est.

1956

Eormula Best Est.

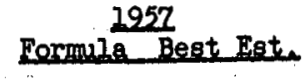

Jan.

Feb.

Maroh

Apr11

May

June

July

Aug.

Sept.

Oot.

Nov:

Deo.
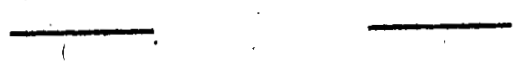

TOTAL

1258 Formula Best Egt. Formula Begt Est.

Jan.

Feb.

March

Apri1

May

June

July

Aug.

Sept.

Det.

Nov.

Dec.

TOTAL,
0.1

1.4

.2

.3

.4

.1

.2

.5

.2

.3

.6

$-.5$

4.8

.3

.1

$.3 * *$

.3

.3

$.4 * *$

.9

.5

.2

.2

.1

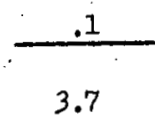

Pre-1954

1954

1955

1956

1957

$1958 \quad 4.8$

1959

3.7

$$
\text { Total }
$$




\section{ESTIMATE OF SY 90 DEPOSITION FROM GUMMED FIIM}

STATION \#- 735

LOCATION STOCKHOLM, SWEDEN

Eormula Best Est. $\frac{1954}{\frac{1955}{2956}}$ Eormula Best Est. Eormula Best Est : Formula Best Est.

Jạn.

Feb.

March

Apri1

May

June

JuIy

Aug .

Sept.

Oet.

.11

Nov.

.15

Deo.

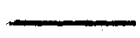

.04

TOTAL

1958

Formula Best Est.

Jen.

0.07

Formuli Best Est.

Feb.

.06

0.15

March

.14

.06

April

1.45

.25

May

.56

June

.85

July

1.46

Aug.

.11

Sept.

.08

.69

1.10

.47

.65

.22

.17

Oct.

.14

.26

Pre-1954

1954

1955

1956

1957

Nov.

.24

.18

Dec.

TOTAL

$\frac{.27}{5.43}$

.13

4.33 
STATION \# 735

1956

Cormula Best Est.

Formula Besit Est.

2956

1956

Tormula Best Eat:

Jan.

Feb.

March

Apri1

May

June

JuIy

Aug.

.2

Sept.

Oot.

Nov,

Deo.
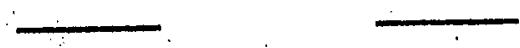

TOTAL

1959

Formile Best Est. Formule Best Est.

Jan.

0.1

Feb.

March

.1

Apr11

.3

May

2.5

1.1

June

1.7

July

4.2

Aug.

Sept.

oct.

Nov.

Dec.

TOTAL

.3

.2

.4

.7

.6

12.2

\section{.3}

.1

.5

1.3

1.9

.8

1.1

.4

.3

.4

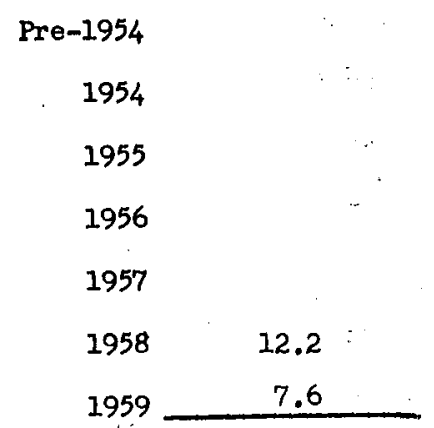

Total

.3

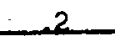

7.6 
ESTIMATE OF Sr 90 DEPOSITION FROM GUMMED FILM

STATION \#- 737

LOCATION DAKAR, FRENCH WEST AFRICA

1954

Eormula Best Est. Formule Best Est. Eormula Best Est. Formula Best Est.

Jan.

Feb.

Maroh

April

May

June

JuIy

Aug.

Sept.

oot.

Nov.

Deo.

TOTAL

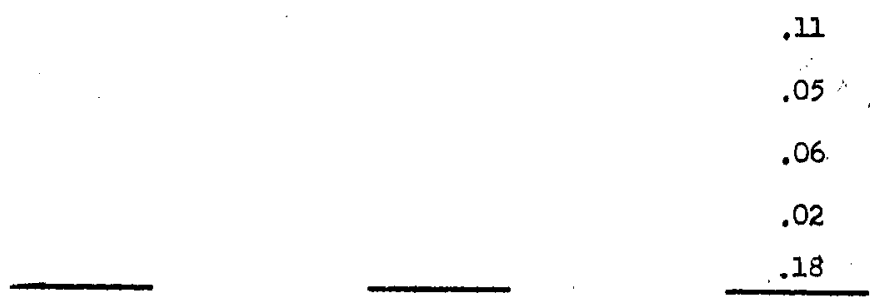

\section{Formule Best.Est. Cormila Best Tat:}

Jan.

Feb.

March

.01

Pre-1954

April

.03

1954

May

.37

1955

June

.42

1956

July

.14

1957

Aug.

1958

Sept.

1959

\begin{tabular}{ll} 
Oct. & .15 \\
Nov. & .18 \\
Dec. & \\
\hline
\end{tabular}

TOTAL 
ESTMMATE OF INFINITY y DOSE FHOM GUMMED FILM

STAIION $\quad 737$

LOCATION DAKAR, FRENCH WEST AFRICA
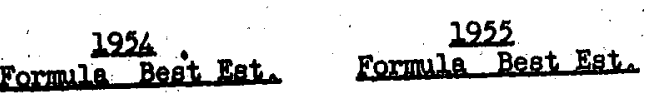

\section{6}

Formula Begt Est.

1957

Formula Best Est.

Jan:

Pëb.

Maroh

Apr11

May,

June

Juzy

Augs:

Sept.

Dot.

Nov.

Deo.

TOTAL

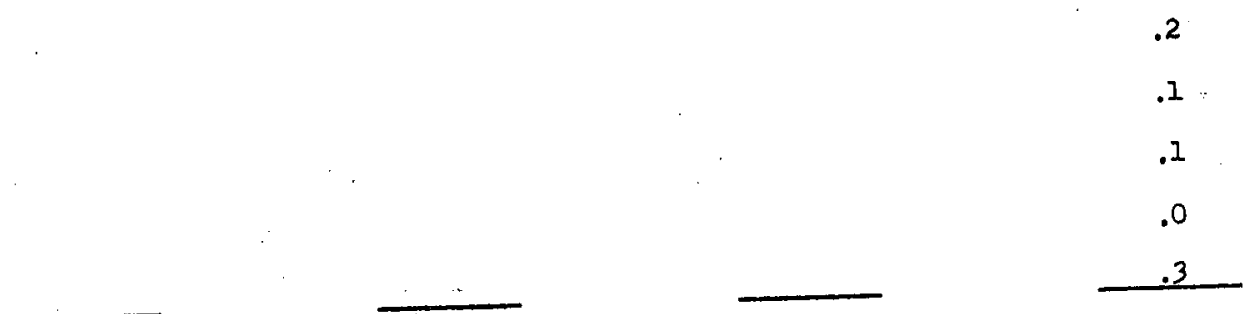

Jan.

Feb.

March

.0

Apri1

.1

May

.7

.8

June

.4

Pre-1954

July

Aug.

Sept.

oet.

.6

1954

1955

1956

1957

1958

1959

Total

Nov. $\quad .5$

Dec.

TOTAL 


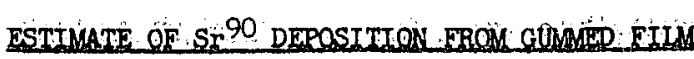

STATION \# 801

LOCATYON_HIROSHIMA, JAPAN

\begin{tabular}{|c|c|c|c|}
\hline 2956 & 2955 & 1956 & 2957 \\
\hline Eoxmula Beet Zat. & Bormula Bert Fet. & Eornula Best Est. & Eornule Best Est. \\
\hline 0.04 & $0.07 *$ & $0.41 *$ & 0.32 \\
\hline .08 & .06 & .21 & .29 \\
\hline .04 & $.13 *$ & $.52 *$ & .48 \\
\hline .03 & $.19 *$ & $.67 *$ & 1.33 \\
\hline .17 & $.22 *$ & .50 & .59 \\
\hline .12 & $.12 *$ & .84 & .23 \\
\hline .11 & $.08 *$ & .23 & .56 \\
\hline .05 & $.06 *$ & .16 & $.30 * *$ \\
\hline .13 & $.05 *$ & .27 & .36 \\
\hline .06 & $.11 *$ & .20 & .30 \\
\hline .26 & .28 & .13 & .09 \\
\hline .05 & .22 & .40 & .23 \\
\hline 1.14 & 1.59 & 4.54 & 5.08 \\
\hline
\end{tabular}

Jan.

Feb.

March

Apr11

Hot:

mene

Juty

Aug.

Sept.

Oot.

Nov.

Dec.

TYIRL

1.14

1.59

Formula Best Eat. Formula Beat Eat.

Jan.

Féb.

March

April

May

June

JuIy

AuE.

Sept.

Uct.

Nov.

Dec.

roril.
0.30

.24

.82

1.83

.52

.35

1.39

.30

.20

.58

.27

.40

7.20
0.85

1.27

2.77

2.39

.99

.57 .

.51

.16

.09

.09

.10

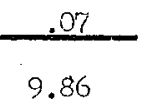

\begin{tabular}{rl} 
Pre-1954 & 1.09 \\
1954 & 1.14 \\
1955 & 1.59 \\
1956 & 4.54 \\
1957 & 5.08 \\
1958 & 7.20 \\
1959 & 9.86 \\
\hline Totbl & 30.5
\end{tabular}




\section{ESTIMATE OF INFINITY y DOSE FROM GUMMED FILM}

STATION \# 801

LOCATION HIROSHTMA, JAPAN

1954:

Formula Best Est.

1955

Eormula Best Est.

2956

$0.1 *$

.1

Feb.

0.0

March

.1

.2*

April

.3

$.4 *$

May

.2

June

1.0

.5

$.4 *$

$.2 *$

JuIy

.3

Aug.

.1

Sept.

-. .3

Oat.

.1

Nov.

Deo.

TOTAL

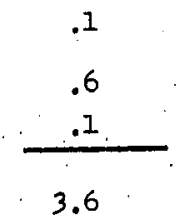

3.6

2.8

$.1 *$

$.1 *$

$.1 *$

.2*

.5

.4

Begt Est.

Formula Best Est.

$0.7 *$

0.6

.3

.5

$.8 *$

.8

$.0 *$

2.5

.8

1.0

1.6

.4

.9

.5

$.6 * *$

.6

.6

.4

.6

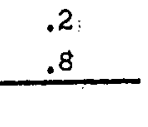

.2

.4

9.1

Jan.

0.5

Feb.

March

April

Nay

June

July

Aug.

Sept.

Oct.

Nov.

Dec.

TOTAL,

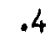

1.6

3.1

1.1

.8

3.7

.8

.5

2.1

.8

$-.9$

16.3
1.8

2.6

5.2

4.3

1.7

.9

.8

.3

.1

.1

Pre-1954 4.2

$1954 \quad 3.6$

$1955 \quad 2.8$

19568.0

$1957 \quad 9.1$

$1958 \quad 16.3$

$1959-18.0$

Total

62
.1

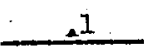

18.0 


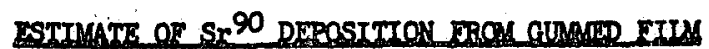

STATION $/ 402$

LOCATSOLL NACASAKI, JAPAN

1954

Eormina Beat Ist.

Jan.

Feb.

Maroh

April

May

June

July

Aug.

Sept.

Oot:

Nor.

Dec.

TOTAL
0.04

.09

.01

.03

.15

.15

.21

.03

.05

.07

.28

.05

1.16
2955

Dormula Beat_ Eat.

$0.46 *$

.06

$.15 *$

$.22 *$

$.13 *$

$.10 *$

$.05 *$

$.03 *$

$.06 *$

$.04 *$

.15

$.24^{\circ}$

1.69
1956

Porquse Best Ist.

$0.76 *$

Eormila Beat Est.

0.93

.51

.36

$.41 *$

$.37 *$

.50

.68

.27

.15

.09

.24

.08

.30

4.10
1.16

.37

.19

.85

.34

.19

.28

.09

.39

5.66
. 2958

Formula Best Ist.

Jan.

Feb.

March

April

May

June

0.38

.32

.69

1.22

.24

.49

July

2.60

Aug.

Sept.

Oct.

Nov.

Dec.

TOTAL
.33

.10

.30

.22

.72

7.61

1959

Eormule Beat Eato

$1.10^{\circ}$

1.09

1.56

2.53

.93

.35

.64

.18

.04

.08

.06

.08

8.64

\begin{tabular}{rl} 
Pre-1954 & 1.38 \\
1954 & 1.16 \\
1955 & 1.69 \\
1956 & 4.10 \\
1957 & 5.66 \\
1958 & 7.61 \\
1959 & 8.64 \\
\hline
\end{tabular}

Total

30.2 


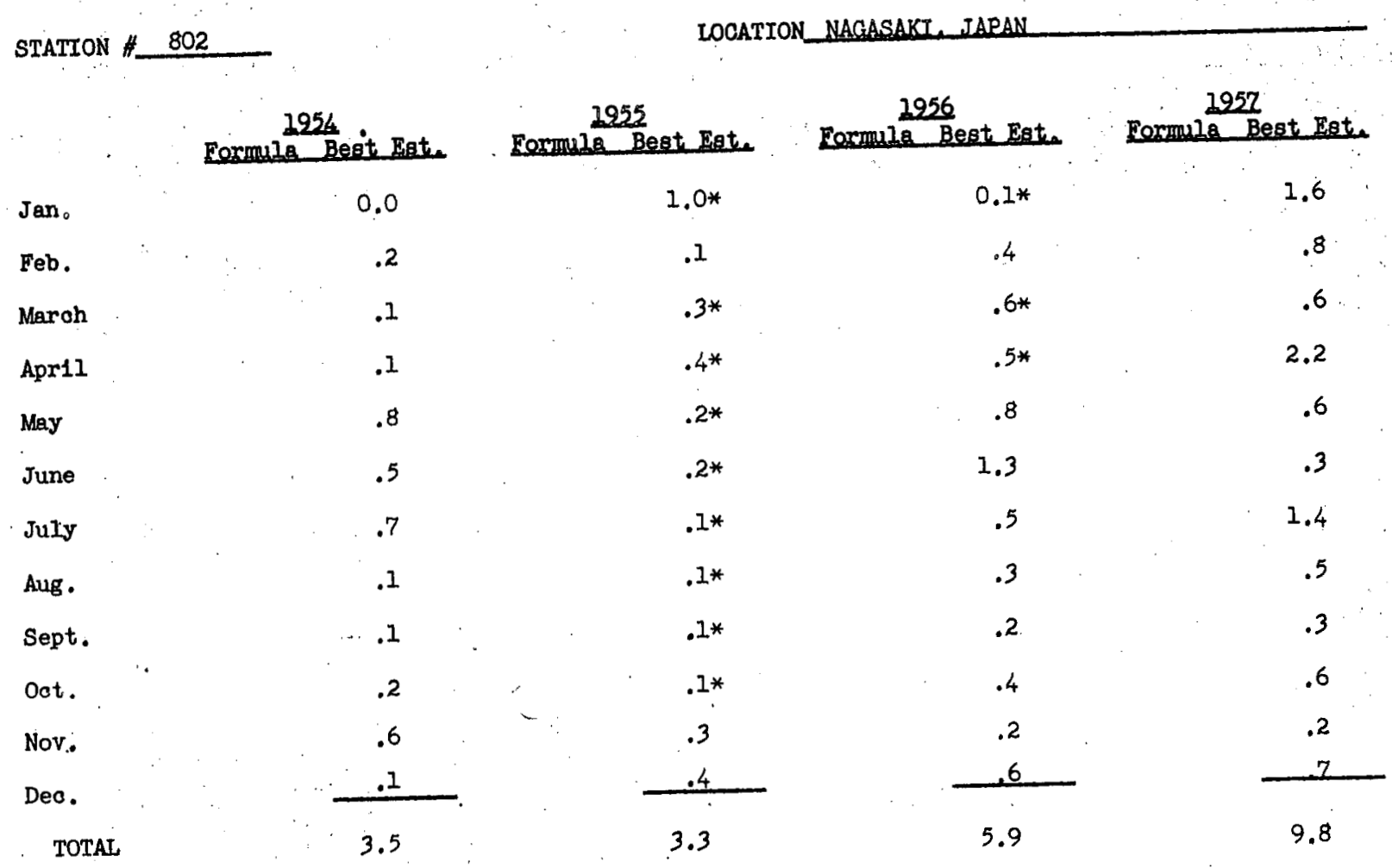

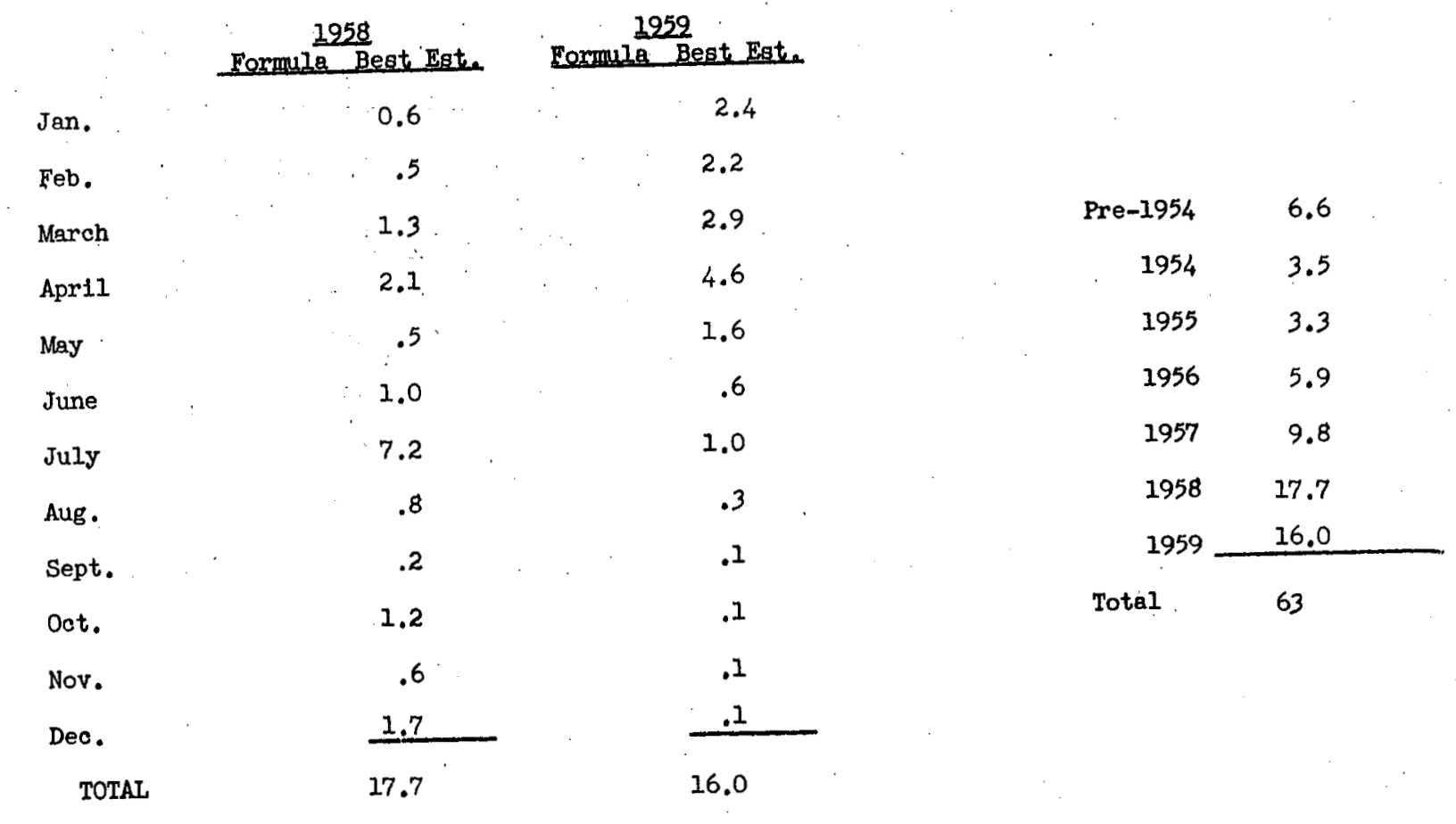




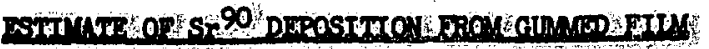

STAFIOA HOL

LOATrOW TAIPEI, TAIWAN

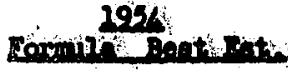

Jan.

pob.

Maroh

Aprig

May

June

July

Aug .

Sept.

Oot:

Nov.

Deo.

TOTAL
0.04

.06

.02

.14

.25

.07

.20

.07

.12

.46

.51

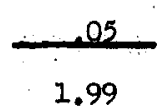

2055 Benouln Bent Brit.

$0.23 *$

.04

$.11 *$

$.27 *$

$.23 *$

$.38 *$

$.14 *$

$.08 *$

$.16 *$

$.62 *$

.61

$\frac{1.02}{3.89}$

Eormun Beat rat

1.10*

.71

$1.08 *$

1.13*

1.52

.85

1.77

.53

.54

$.19 * *$

1.19

$.22 *$

10.83
2957

Dormuze Best Eat.

0.87

1.96

1.31

1.45

.68

.17

.19

.31

.54

.27

.29

.40

8.44

19988

2939

Formuli Beativat. Cormule Bast Bat.

Jan.

0.72

1.12

Réb.

.76

1.26

March

.53

1.18

April

.51

1.44

May

.54

.24

June

.53

.54

July

1.82

.18

Aug.

.18

.23

Sept.

.15

oct.

.48

.20

.16

\begin{tabular}{rr} 
Pro-1954 & -- \\
1954 & 1.99 \\
1955 & 3.89 \\
1956 & 10.83 \\
1957 & 8.44 \\
1958 & 7.76 \\
1959 & 6.79 \\
\hline
\end{tabular}

Nov.

.70

.13

Deo.

Toral

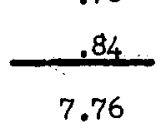



6.79 


\section{ESTINATE OF INFINITY $y$ DOSE FROM GUMMED FILM}

STATION \# 804

IOCATION TAIPEI_ TATWAN

1954

Dormila Bagt Fat.

$\begin{array}{lc}\text { Jan. } & 0.0 \\ \text { Feb. } & .1 \\ \text { Maroh } & .1 \\ \text { Aprt1 } & .7 \\ \text { May } & 1.2 \\ \text { June } & .3 \\ \text { July } & .7 \\ \text { Aug. } & .2 \\ \text { Sept. } & 1.1 \\ \text { Oot. } & 1.2 \\ \text { Nov. } & .1 \\ \text { Deo. } & 6.0 \\ \text { TOTAL } & \end{array}$

2955 Eormula Best Est. $0.5 *$

.1

$.2 *$

$.5 *$

$.4 *$

$.7 *$

$.2 *$

$.1 *$

$.3 *$

$1.0 *$

1.1

1.8

6.9 20rmule Best Est.

$1.8 *$

1.2

$1.6 *$

$1.7 *$

2.4

1.7

4.9

1.2

1.2

$.4 * *$

2.3

$.5 * *$

20.9
2957 Bormula Beat bot.

1.5

3.3

$2.1 \cdots$

2.7

1.2

.3

.3

.5

.8

.6

.6

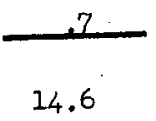

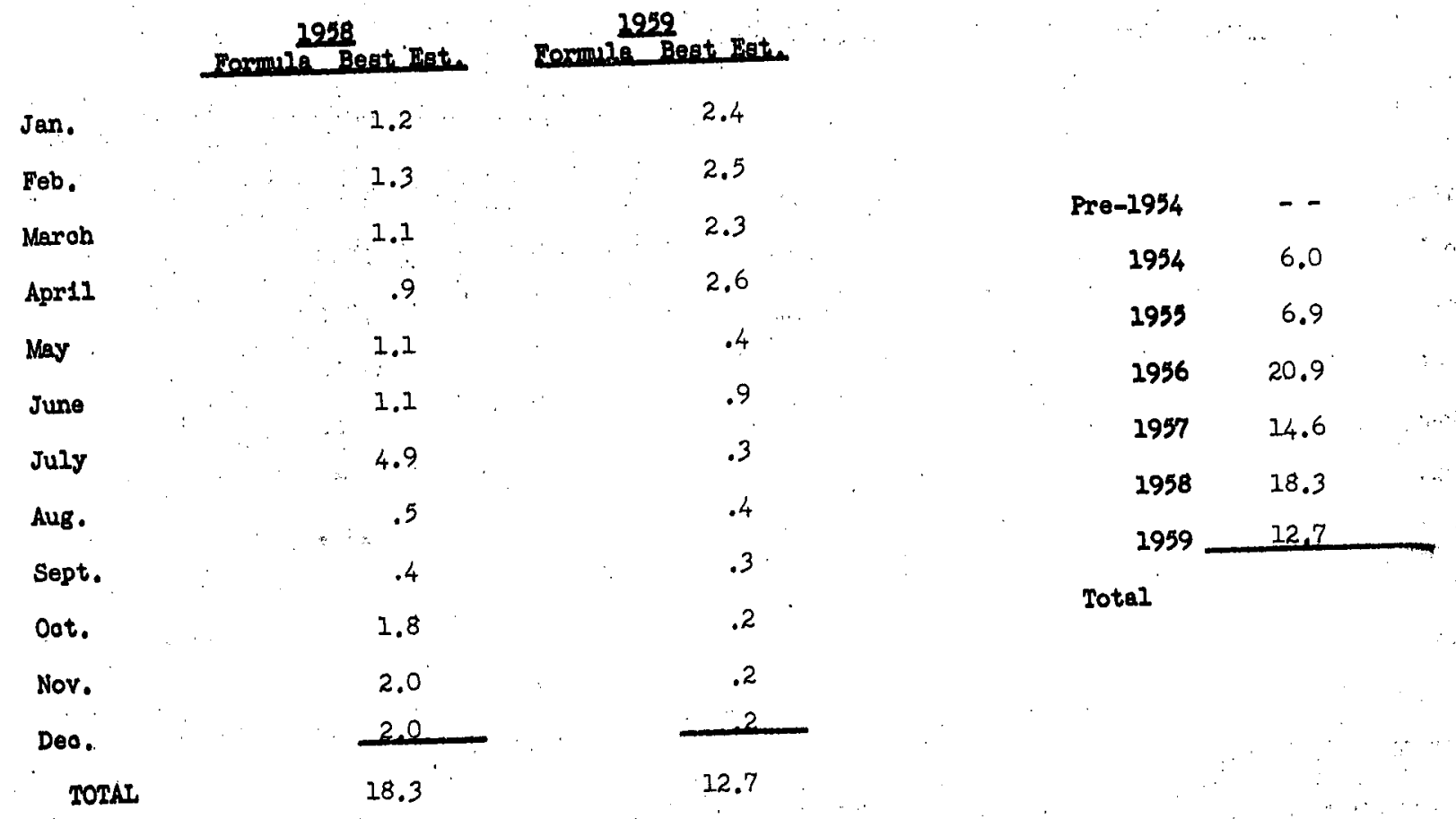




\section{ISTLMATE OF Sr ${ }^{90}$ DEPOSITION WROM GUMMED FILM}

STAHON 805

LOCATION TOKYO, JAPAN, HANEDA AFB

1954

1955

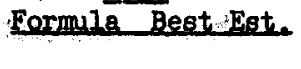

Eormula Best Est.

1956

1957

$\begin{array}{lllll}\text { Jan. } & 0.04 & 0.06 * & 0.60 * & 0.27 \\ \text { Feb. } & .09 * * & .11 & .34 & .75 \\ \text { March } & .13 * * & .31 * & .54 * & .70 \\ \text { Apr11 } & .03 & .41 * & .54 * & 1.13 \\ \text { May } & .20 & .28 * & .44 & .90 \\ \text { June } & .09 & .26 * & .37 & .58 \\ \text { JuIy } & .09 & .10 * & .26 & .86 \\ \text { Aug. } & .12 & .12 * & .30 & .35 \\ \text { Sept. } & .23 & .14^{*} & .55 & .61 \\ \text { Oct. } & .29 & .49 * & .70 & .41 \\ \text { Nov. } & .31 & .22 & .33 & .15 \\ \text { Dec. } & .37 & .24 & .20 & .27 \\ \text { TOTAL } & 1.99 & 2.74 & 5.17 & 6.98\end{array}$

TOTAL

1.99

.17

6.98

Formula Best Est. Formulo Besti2Est.

Jan.

0.23

0.45

Feb.

.25

.94

March

3.24

1.85

Apri1

2.20

2.07

May

1.00

2.16

1.42

July

.43

.98

Aug.

.54

.36

Sept.

.25

.16

\begin{tabular}{rr} 
Pre-1954 & 0.83 \\
1954 & 1.99 \\
1955 & .2 .74 \\
1956 & 5.17 \\
1957 & 6.98 \\
1958 & 9.83 \\
1959 & \\
\hline
\end{tabular}

oct.

.24

.50

Total

Nov.

.53

Dec.

TOTAL

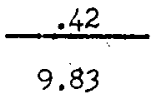


STATION \# 805

LOCATION TOKYO, JAPAN, HANEDA AFB

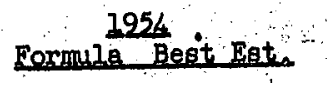

0.0

Jan,

Feb.

March

Aprt1

May

June

July

Aug.

Sept.

Oot.

Nov.

Deo.

TOTAI
. $.2 * *$

$.3 * *$

.2

1.1

.3

.3

.3

.6

.7

.7

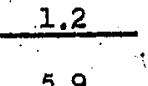

1955

Formula Best Est. $0.1 *$

.2 $.6 *$

$.7 *$

$.5 *$

$.5 *$

$.2 *$

$.2 *$

$.2 *$

$.8 *$

.4

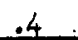

4.8

2956 Formula Best Est,

Formula Best Eat.

$1.0 *$

.5

$.8 *$

$.8 *$

.7

.7

.7

.6

1.2

1.3

.6

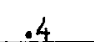

9.3
0.5

1.3

$1.1 \ldots$

2.1

1.5

$1.0 \ldots$

1.4

.5.

1.1

.8

.3

.5

12.1

1958

Formuli Best Eat. Eormula Best.Est.

Jan.

Feb.

Maroh

Apri1

May

June

July

Aug.

Sept.

oot.

Nor.

Deo.

TOTAL
0.4

.4

6.3

3.8

2.1

.8

1.5

.7

.5

1.9

1.5

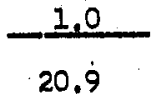

1.0

1.9

3.5

3.7

3.8

2.4

1.6

.6

.3

\begin{tabular}{rr} 
Pre-1954 & 3.2 \\
1954 & 5.9 \\
1955 & 4.8 \\
1956 & 9.3 \\
1957 & 12.1 \\
1958 & 20.9 \\
1959 & \\
\hline
\end{tabular}

Total 


\section{4}

Tommin Bantsat

Jain.

Feb.

Maroh

Apri1

May

June

JuIy

Aug.

Sept.

Oot.

Nov.

Deo.

TOTAI
0.04

.11

.35

.02

.18

.08

.08

.07

.22

.27

.29

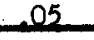

1.76

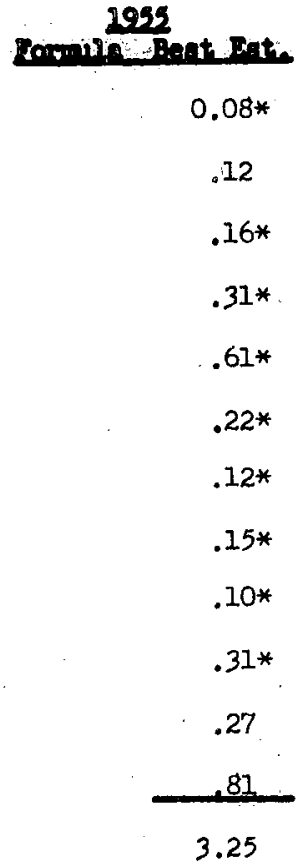

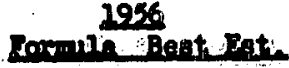

$0.48 *$

.29

$1.16 *$

$.55 *$

.60

.22

.51

.23

.35

.39

.34

22

5.34
1957

Rormin Begt Eat.

0.43

.50

.51

1.01

.58

.29

.57

.39

.56

.72

.18

41

6.15

1958

Formun Bent Dat.

Jan.

0.22

0.79

Feb. $.27 * *$

March

1.41

2.66

1.10

May

.39

June

.37

July

.50

.49

Sept.

.95

Oet.

.92

Nov.

1.02

Dec.

Torat.

10.30

\begin{tabular}{cc} 
Pre-1954 & 0.68 \\
1954 & 1.76 \\
1955 & 3.25 \\
1956 & 5.34 \\
1957 & 6.15 \\
1958 & 10.30 \\
1959 & \\
\hline
\end{tabular}

Totel 
STATION 806

IOCATIOR MTSAMA JAPAN

Fomula Bosit Inatio

$\operatorname{Jan} 0$

Feb.

Marah

Apri1

May

June

Juty

Aug.

Bept.

oot.

Nov:

Deo.

TOTAT
0,0

.2

.6

.1

1.0

.3

.3

.1

.6

.7

.7

.1

4.7
1955

Rormun: Bagt Rati.

$0.2 *$

.2

$.3 *$

$.6 *$

$1.1 *$

$.4 *$

$.2 *$

$.2 *$

$.2 *$

$.5 *$

.4

1.4

.5 .7
2956 Dopmina Begt Pat.

$0.8 *$

.5

$1.8 *$

$.8 *$

1.0

.4

1.1

.5

.7

.7

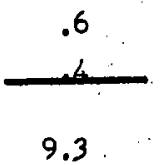

9.3

\section{7}

Bormina Beat Bat.

0.8

.8

.8

1.9

1.0

.5

.9

.6

.9

1.6

.4

7

10.8

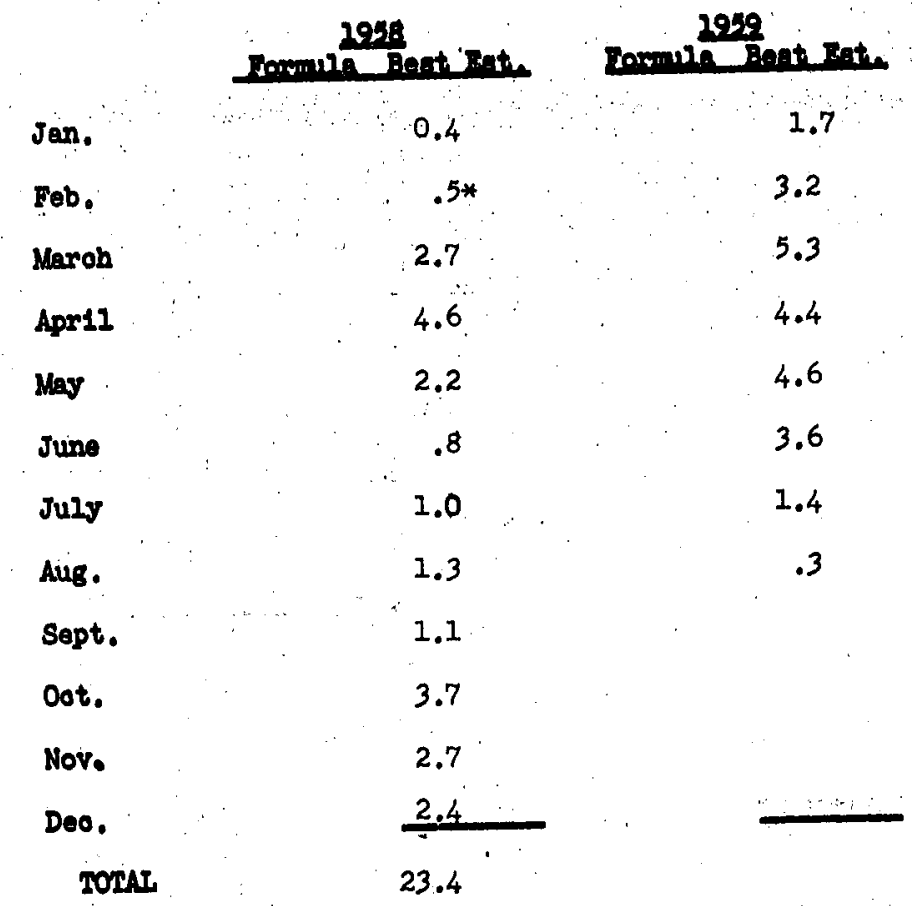

\begin{tabular}{rr} 
Pro-1954 & 2.6 \\
1954 & 4.7 \\
1955 & 5.7 \\
1956 & 9.3 \\
1957 & 10.8 \\
1958 & 23.4 \\
1959 & \\
\hline
\end{tabular}

Total 


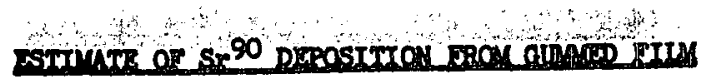

STATION 810

rOGATIOA METBOURNE AUSTRAITA

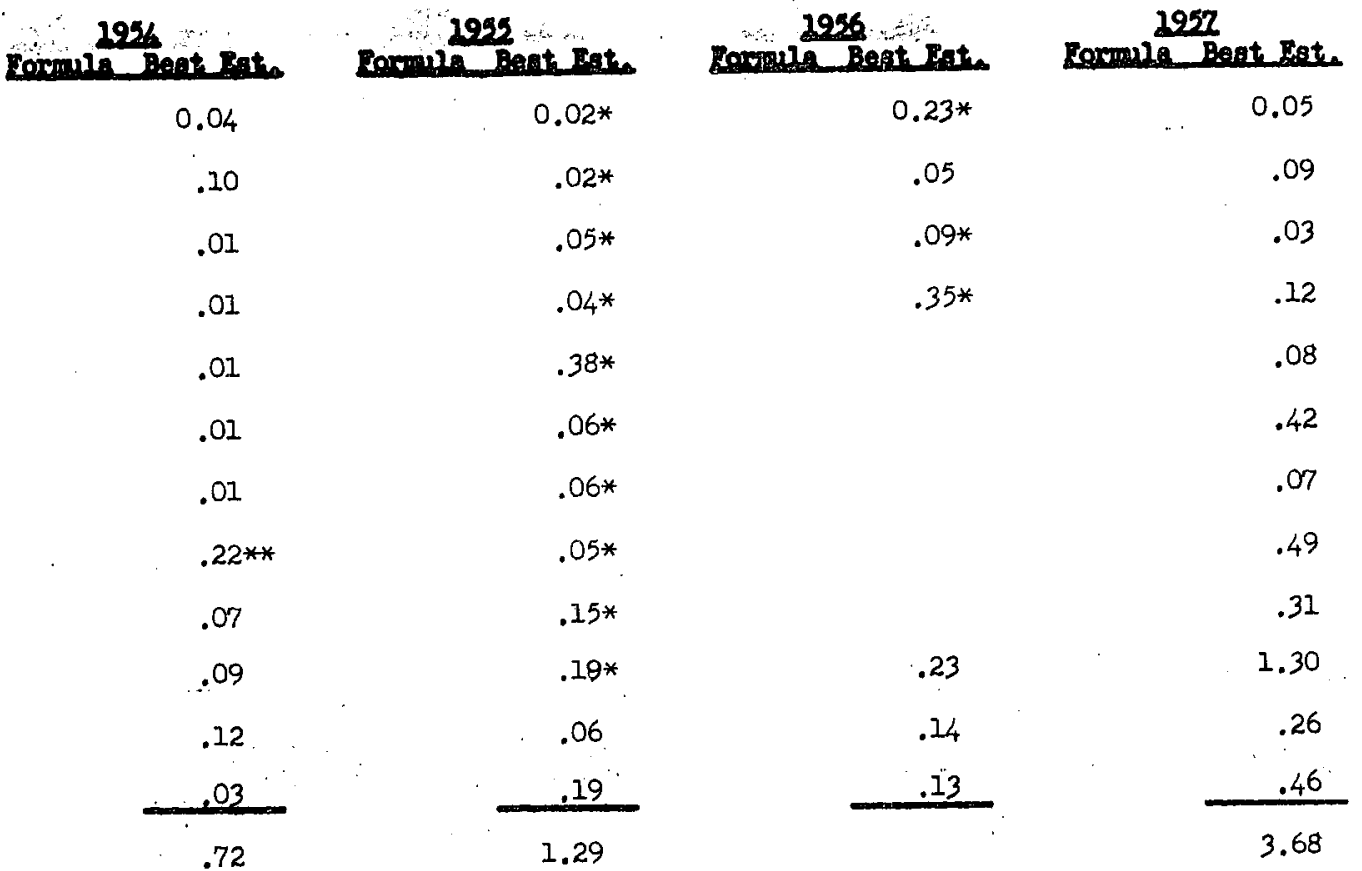

TOTAL

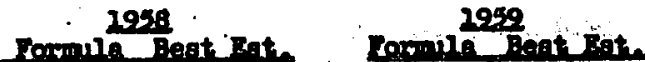

0.06

Jan.

.10

March

.04

April

May.

June

July

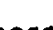

Aug.

1959

Sept.

1959

Dot.

Total

Nor.

Deo.

rorit. 
1254
Formilo Best-Eat.

Jan.

Feb.

Maroh

Apri1

Mey

June

July

Aug .

Sept.

Oct.

Nov.

Deo.

TOTAC

$$
.0
$$

.2

.0

.0

.1

.0

.0

$.5 * *$

2

.2

.3

1

1.6

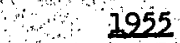

Eormule Best Est.

$0.0 *$

$.0 *$

$.1 *$

$.1 *$

$.7 *$

$.1 *$

$.1 *$

$.1 *$

$3 *$

.3*

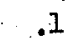

.3

2.2 $\begin{array}{r}1957 \\ \hline\end{array}$

Formula Best Est.

Formule Best Est.

$0.4 *$

0.1

.1

$.1 *$

$.5 *$

$\because 2$

.0

.2

.1

.1

8

1

.8

.5

.4

2.6

.3

.5

2

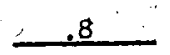

6.7
1958

Formula Begt Este

Jan.

Feb.

March

Apri1

May

June

Ju1y

Aug.

Sept.

Oct.

Nov.

Dec.

TOTAL

\section{Formila Best Est}

0.1

.2

.1 
STATION 811

LOCUIOL WELLINGTON, NEW ZEALAND

1995

2956

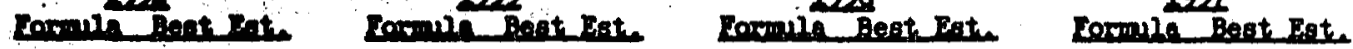

Jain.

0.04

$0.03 *$

$0.14^{*}$

0.07

Feb.

.05

$.03 *$

.04

.03

Maroh

.01

$.06 *$

.22*

.07

Apr11

.00

$.07 *$

.06*

.07

May

.01

June

.02

July

.05

$.28 *$

$.03 *$

$.04 *$

.02

.12

Aug.

.03

$.04 * *$

.06

.10

Sept.

.12

oot:

.20

Hov.

.05

.02*

$.04 *$

.05

.03

.32

Dec.

TOTAL

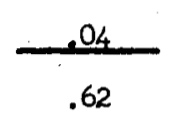

.05

.74

.03

.12

$.14 * *$

.

$.17 * *$

.27

.08

.06

1.54

\section{8}

Dewir Beat Bot.

1959

Sorula Bent Rat.

$$
0.07
$$

0.05

ser.

.19

.07

Maroh

.06

.07

April

.07

.06

un

.13

June

.03

.17

Juls

.06

Aug.

.03

.76

.14

.12

.08

Sept.

.07

$\frac{.07}{.82}$

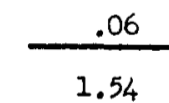

Dat.

.04

Nor.

.02

Deo.

.06

Toral

.93

\begin{tabular}{rr} 
Pre-1954 & 0.78 \\
1954 & .62 \\
1955 & .74 \\
1956 & .82 \\
1957 & 1.54 \\
1958 & .93 \\
1959 & \\
\hline
\end{tabular}

Total 


\section{ESTIMATE OF INFINITY $\not$ DOSE FROM GUMMED FILM}

STATION \# 811

IOSATION WELIINGTON, NEM ZZEATAND

2254. Eormula Begt Esto

Jan,

Eormula Best Est.

1926

Formule Best Est. : Formula Best Est.

Feb.

Maroh

April

May

June

JuIy

Aug.

Sept.

Oot.

Nov.

Deo.

TOTAL

0.0

$0.1 *$

.1

$.1 *$

.1

.0

.0

$0.2 *$

.1

$.1 *$

$.1 *$

$.5 *$

$.1 *$

$.1 *$

$.1 * *$

.0*

$.1 *$

.1

.1

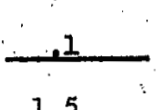

1.5
.3

5
$.3 *$

$.1 *$

.0

.1

.1

.1

.1

.1

.2

1.5

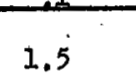

1958

Fomula Beat Eat.

0.1

Jen.

Feb.

March

Apr11

May

June

July

Aug.

Sept.

Oct.

Nov.

Dec.

TOTAL
1959

Eormula Beet Eat.

0.1
.1
.1
.1
.3
.2
.2
.1
.1

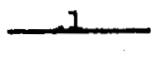

0.1

.1

.1

.1

.2

.2

.5

.2

$.3 * *$

$.4 * *$

.5
1

2.8 


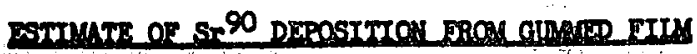

STATION

roastor COLOMBO, CEYLON

2935

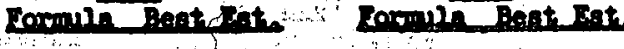

$0.02 *$

1936

1957

Jain.

0.04

Cormuln Bept Iat.

Tormule Beat Eat.

Feb.

.04

$0.15 *$

0.06

Maroh

.27

April

.15

$.02 *$

.03

.07

May

$2.37 \quad .74$

$.07 *$

$.11 *$

.12

$.05 *$

$.21 *$

.07

June

.11

$.13 *$

.06

.23

JuIy

$.13 *$

.14

JuIy

.12

$.23 *$

Aug .

.10

$.03 *$

Sept.

.04

$.04 *$

oot:

.08

$.10 *$

Nov.

.04

.04

.07

.20

Deo.

05

.04

TOTAL

1.78

0.90

.12

.19

.12

.12

.17

.09

.09

.05

.07

1.28

.27

1.61

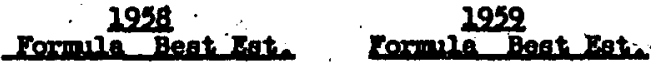

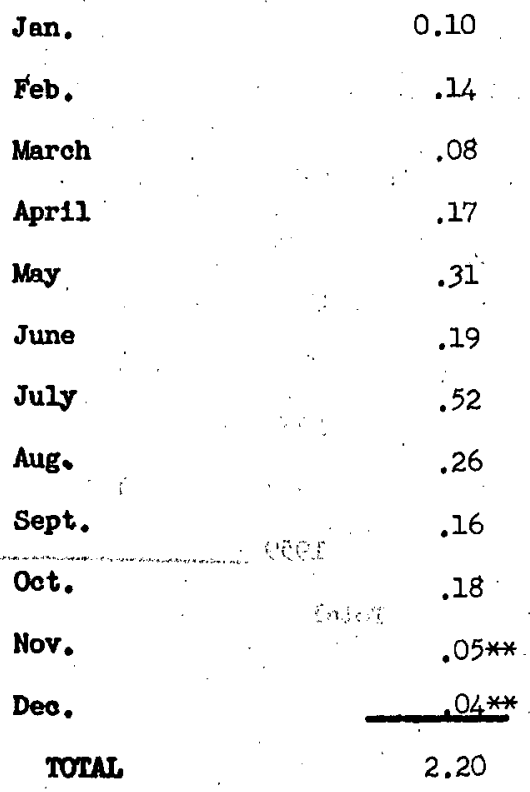

1.67

.51

.82

.15

Pre-1954

1.32

2954

1.78

1955

0.90

1956

1.28

1957

1.61

1958

2.20

1959

Total 
STAIION \#- 813

LOCATION COLOMBO, CEYLON

$1954 \quad 1955$ Formule Best Est.

Jan.

Feb.

Maroh

April

May

June

July

Aug .

Sept.

oct.

Nov:

Deo.

TOTAT.
0.0

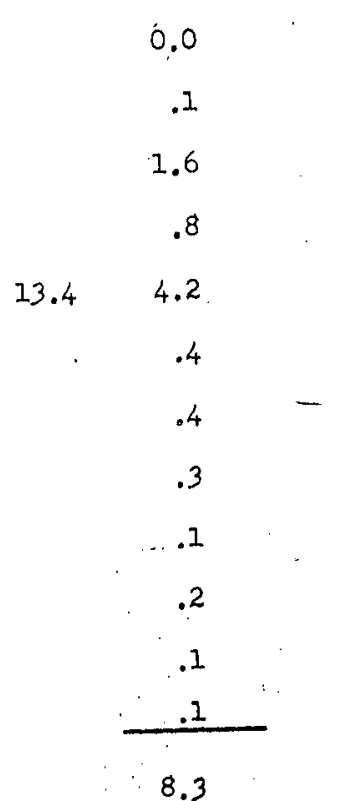

8.3 Formula Best Est.

$$
0.1 *
$$

$.0 *$

$.1 *$

$.1 *$

$.2 *$

$.2 *$

$.4^{*}$

$.1 *$

.1*

$.2 *$

.1

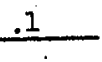

1.7
1956 Eormula Best Est.

$0.2 *$

.1

$.2 *$

.3*

.1

.1

.3

.2

.3

.3

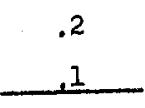

2.4
2957

Formule Best Este

0.1

.1

.2

.1

.4

.3

.3

.3

.2

.2

.1

5

2.8

Formula Beat Eata Formula Best Eate

Jan.

$$
0 . \ddot{2}
$$

Feb. 2

March

.2

Apr11

.3

May

.6

June

.4

2.9

.9

July

1.4

1.3

Aug.

.7

.2

\begin{tabular}{rr} 
Pre-1954 & 5.8 \\
1954 & 8.3 \\
1955 & 1.7 \\
1956 & 2.4 \\
1957 & 2.8 \\
1958 & 5.1 \\
1959 & \\
\hline
\end{tabular}

Sept.

.4

oct.

.5

Total

Nov.

. I**

Dec.

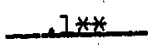

TOTAL 


\section{ESTIMATE OF SF 90 DEPOSITTON FROM GUMMRD EIUM}

STATION " 814

LOCATIOK SINAAPORE

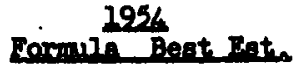

Jan.

Teb.

March

April

May

June

JuIy

Aurg.

Sept.

Dot.

Nov.

Deo.

TOTAL
0.04

.06

.31

.13

.44

.06

.07

.08

.07

.03

.05

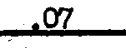

1.41

$\frac{1955}{\text { Bermula Best Dst. }} .05 * *$
$.03 * *$
$.00 * *$
$.09 * *$
$.15 * *$
$.08 * *$
$.07 * *$
$.04 *$
$.20 *$
$.03 *$
$.09 * *$
$.06 * *$
.99

Bormula Best Dat.

$0.10 *$

.03

$.24 *$

$.06 *$

.05

.19

.24

.09

.08

.14

.09

$.08 *$.

1.29
1957

Formule Best Est.

0.03

.08

.04

.04

.07

.05

$.19 * *$

$.21 * *$

.15

.11

.04

$28 * *$

1.29

\section{8}

Pormila Beat Bat. Bomula Beat Bate

Jan.

0.06

0.23

Feb.

.10

.26

March

.12

.15

Apr11

.07

.17

May

$\because 05$

June

.15

July

1.71

Aug.

$.35 * *$

sept.

$.25 * *$

oot.

.04

Nov.

.08

Deo.

$.17 * *$

TOTAL

3.15

\begin{tabular}{rr} 
Pre-1954 & 1.32 \\
1954 & 1.41 \\
\hline 1955 & .99 \\
1956 & 1.29 \\
1957 & 1.29 \\
1958 & 3.15 \\
1959 & \\
\hline
\end{tabular}

Total 
ESTIMATE OF INFINITY $y$ DOSE FROM GUMMED FILM

STATION \# 814

IOCATION SINAAPORE

Formula Best Est.

Cormule Best Ist.

Formulio Begit Erit.

2957

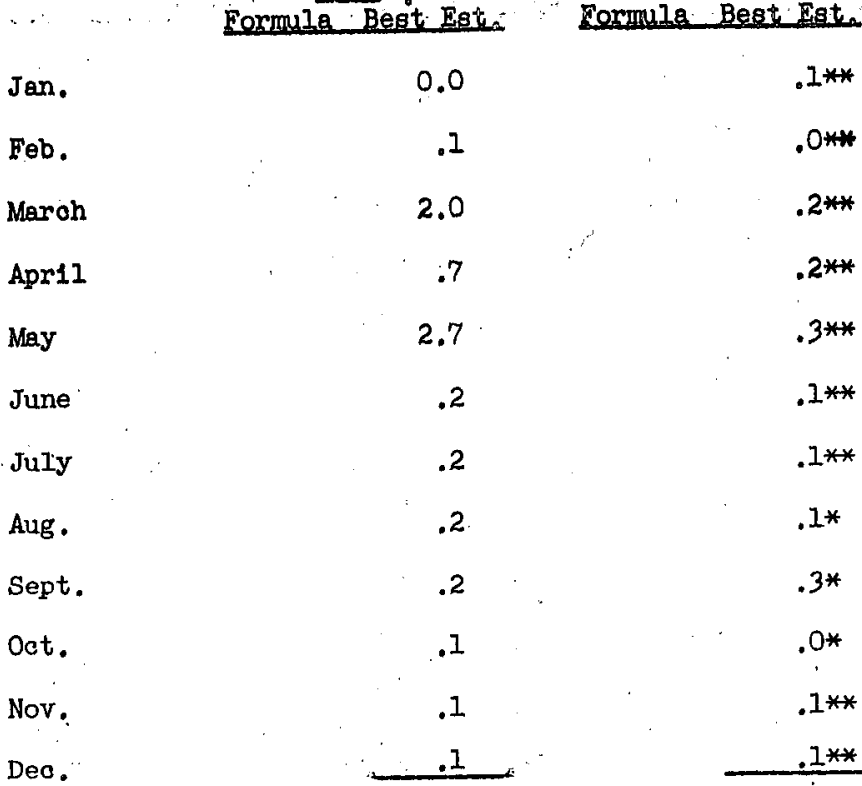

$0.2 *$

.1

**

$.4 *$

**

$.1 *$

***

***

*

3*

TOTAL

Formule Best Est

Formula Best Est.

Jen.

0.1

0.5

Feb.

.2

.5

March

.2

.3

April

.7

May

.1

June

.3

July

4.4

.3

.1

.4

.2

.2

.3

.2

0.1

1

.1

.1

.1

.1

$.3 * *$

$.3 * *$

2

.2

.1

5**

sts

\section{(1)}

1

1

1

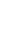




\section{ESTIMATE OF Sr 90 DEPOSITION FROU GUMUD EJLM}

STATION \# 815

LOCATION SYDNEY, AUSTRALIA

155

1955

2956.

2957

Eormula Best. Bste. Formula Best Esth Eormula Best Est. Formulo Best Est.

Jan.

0.04

$0.10 *$

$0.18 *$

0.07

Feb:

.05

$.04 *$

.10

.10

March

.01

$.06 * *$

.11*

.10

April

.00

May

.02

June

.01

JuIy

.01

$.06 *$

$.41 *$

$.07 *$

.04

.05

.02

Aug.

.56

$.04 * *$

.07

.04

$.05 * *$

.05

.02

Sept.

$.05 * *$

.03

.11

oct.

.15

.09**

.02

.12

Nov.

.09

$.08 *$

Dee.

.10

.08

.23

.19

.20

TOTAL

.03

1.07

1.19

\begin{tabular}{c}
.11 \\
.12 \\
\hline 1.09
\end{tabular}

.05

.67

1.54

$1958 \quad-1259$

Formilia Best Eate Formula Best Eot.

Jan.

0.12

0.10

Feb.

.20

.11

March

.04

.09

April

May

.03

.10

June

.15

June

.04

.29

July

.02

Aug.

.06

Sept.

.12

Oct.

.07

Nov.

$.04 * *$

$\begin{array}{rr}\text { Pre-1954 } & 1.32 \\ 1954 & 1.07 \\ 1955 & 1.19 \\ 1956 & 1.09 \\ 1957 & 1.54 \\ 1958 & .85\end{array}$

1959

Total

Dec.

TOTAL

.04

.07

.85 
ESTIMATE OF INFINITY y DOSE FROM GUMMED FILM

STATION \#- 815

LOGATION SYDNEY, AUSTRALIA

\begin{tabular}{|c|c|c|c|c|c|c|}
\hline & Eormul & 1256 Best Est. & Eonmula & $\frac{55}{\text { Best Est. }}$ & Eormula Best Est. & Eormule Best Est. \\
\hline Jan. & & 0.0 & & $0.2^{*}$ & $0.3^{*}$ & 0.1 \\
\hline Feb. & & .1 & .. & $.1 *$ & .2 & .2 \\
\hline March & & .0 & & $.1 * *$ & $.2 *$ & .2 \\
\hline Apr11 & & .0 & & $.1 *$ & $.1 *$ & .1 \\
\hline May & & .1 & & $.7 *$ & .1 & .0 \\
\hline June & & .0 & & $.1 * *$ & .1 & .1 \\
\hline July & & .0 & & $.1 * *$ & .1 & .0 \\
\hline Aug. & & .8 & & $.1 * *$ & .1 & .2 \\
\hline Sept. & & .4 & & $.1 * *$ & .0 & .2 \\
\hline Oct. & & .2 & & $!^{1 *}$ & .4 & .4 \\
\hline Noṿ. & & .2 & & .1 & .2 & .7 \\
\hline Deo. & & .0 & & .2 & .2 & 1.2 \\
\hline TOTAL & & 1.8 & & 2.0 & 2.0 & 2.8 \\
\hline . & & $\therefore$ & & . & & \\
\hline
\end{tabular}

$1952:$

Formula Best Est. Formula Best Est.

Jan.

Feb.

March

April

May

June

July

Aug.

Sept.

Oct.

Nov.

Dec.

TOTAL
0.2

.3

.1

$\because 0$

.1

.4

.1

.3

.2

$.2 * *$

.1

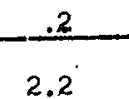

0.2

.2

.2

.2

.3

.5

\begin{tabular}{rr} 
Pre-1954 & 5.8 \\
1954 & 1.8 \\
1955 & 2.0 \\
1956 & 2.0 \\
1957 & 2.8 \\
1958 & 2.2 \\
1959 & \\
\hline
\end{tabular}

Totel 
ESTIMATE OF $3 r^{90}$ DEPOSITION FROM GUMMED FIIM

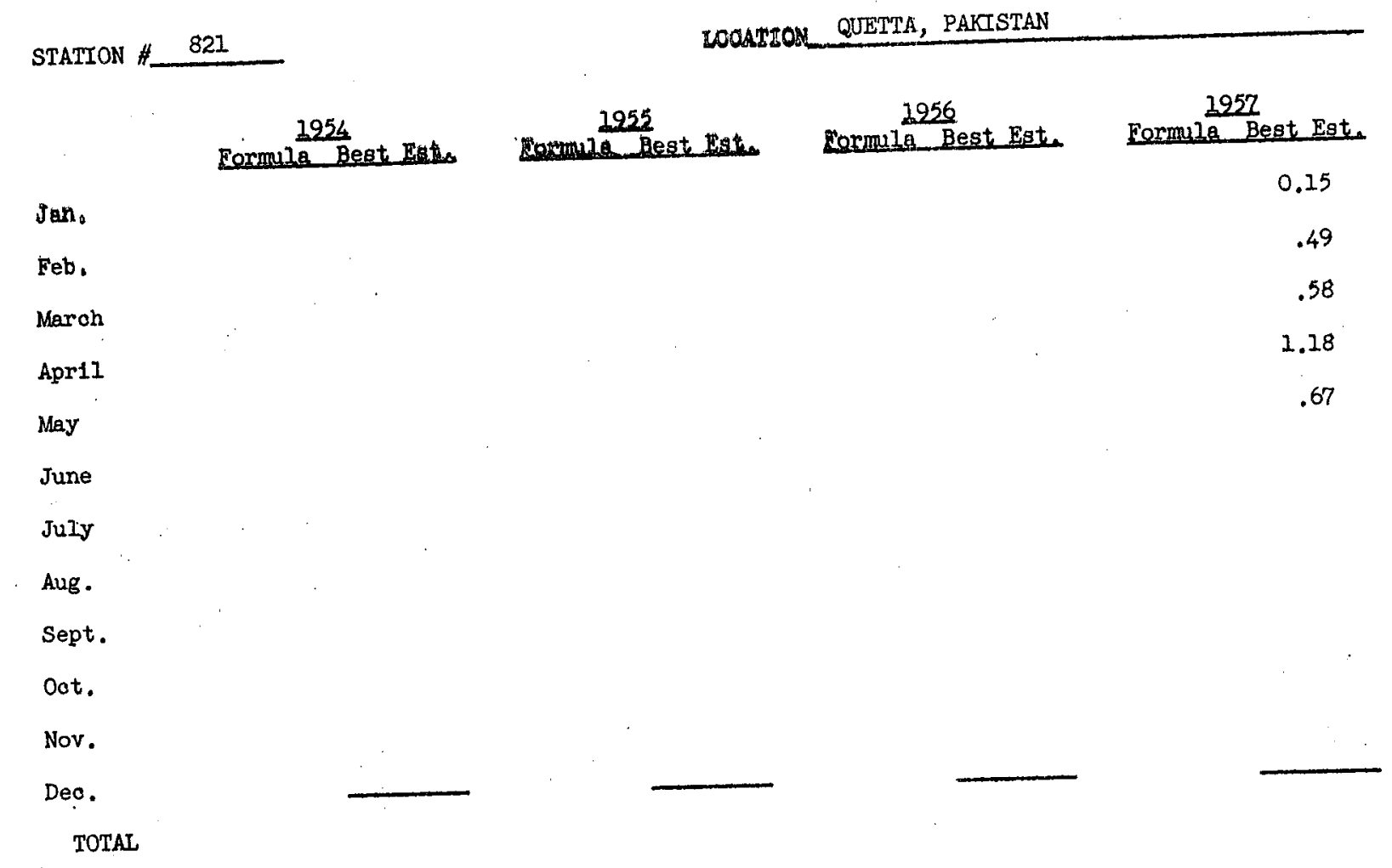

1958

Formila Best Est. Formula Best Est.

Jan.

Feb.

Maroh

Apr11

May

June

July

Aug .

Sept.

Oct.

Nov.

Dec.

TOTAI
1.15

1.90

1.17

1.82

1.17

.56

.38

.32

.19

.11

.08

.12

.57

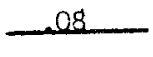

8.93
Pre-1.954

1954

1955

1956

1957

1958

$1959+8.93$

Total 
ESTIMATE OF INFINITY $\gamma$ DOSE FROM GUMMED FIIM

STATION \# 821

LOCATION QUETTA, PAKISTAN

1954

1955

Formula Best Eato Eormula Best Est. Rormula Best Esta

Jan。

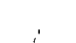

1957

Feb.

Maroh

Apr11

Mey

Formula Best Est:

0.3

.8

.9

2.2

1.1

June

July

Aug.

Sept。

Oct.

Nov。

Dec.

TOTAL

Formula Best Est

Jan.

Feb.

March

Apr11

May

June

July

Aug.

Sept.

Oct.

Nov.

Dec.

TOTAL
1959

Formula Best Est.

2.4

3.8

2.2

3.4

Pre-1954

1954

1955

1956

1957

1958

$1959 \square-16.6$

Tota1
.1

16.6 


\section{ESTIMATE OF Sr 90 DEPOSITION FROM GUMMED FILM}

STATION \# 822

LOCATION PESHAWAR, PAKTSTAN

. 1954

Formula Best Est. Eormula Best Est

Jan.

Feb。

Maroh

April

May

June

JuIy

Aug.

Sept.

Oot.

Nov.

Deo.

TOTAL
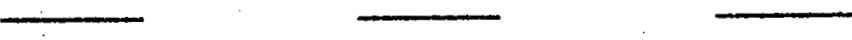
STATION \# 822

\section{4} Formula Besit Est.
1955

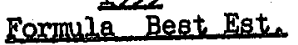

LOCATION PESHAWAR, PAKISTAN

1956

Cormula Best Est.

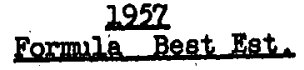

Jan,

Teb.

March

Apri1

May

June

JuIy

Aug.

Sept.

oot.

Nov:

Deo.
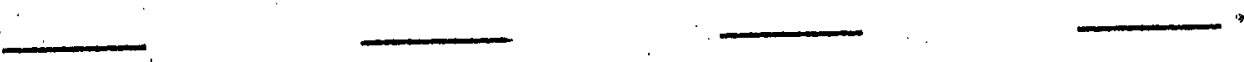

TOTAL

1958

Formula Best Est.

Jan.

Feb.

March

Apri1

May

June

July

Aug.

Sept.

Oet.

Nov.

Dec.

TOTAL

.3

.9

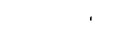

1959

Formula Best Est.

2.9

4.1

$2.4 * *$

3.1

6.8

2.7

1.4

.6

.6

.5

.1

.2

25.4
Pre-1954

1954

1955

1956

1957

1958

1959

25.4

Total 


\section{ESTIMATE OF"SI 90 DEPOSITION FROM GÜMMED FILM}

STATION \#_.823

LOCATION KARACHI, PAKISTAN

2925

Formula Best Est. Formila Best Est.

1956

1957

0.06
0.04

Jan.

.15

Feb.

.14

March

.22

Apri1

May

June

July

Aug .

Sept.

Oct.

Nov.

Dec.

TOTAL

Formula Best Est.

Jan.

1959

Feb.

.25

March · . . .12

April.

.19

May

.41

June

.30

July

.31

Aug.

.21

Sept.

.13

Oct.

.04

Nov.

.10

Dec.

.30

Formule Best Est.

0.32

.39

$1.25 * *$

$1.41 * *$

Pre-1954

.40

1954

1955

1956

.44

.41

1957

.31

1958

.26

1959

5.55

Total

.11

.15

.10

5.55 
ESTIMATE OF INFINITY $y$ DOSE FROM GNMMED FILM

STATION \#_ 823

LOCATION KARACHI, PAKISTAN
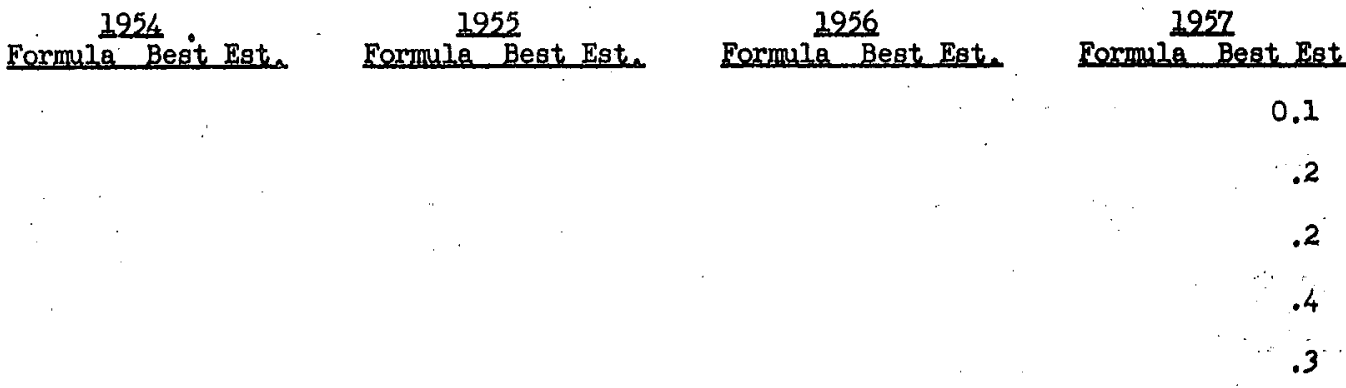

May

June

July

Aug.

Sept.

Dot.

Nov:

Deo.

TOTAL

Formula Best Est. Formula Best Est.

Jan.

Feb.

March

Apri1

May

June

July

Aug.

Sept.

Oct.

Nov.

Dec.

TOTAL
.4

.2

.3

.8

.6

.8

.6

.3

.i

.3

.7
0.7

.8

$2.4 * *$

$2.6 * *$

.7

.7

.7

.5

.4

.2

.2

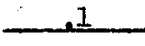

10.0
Pre-1954

1954

1955

1956

1957

1958

$1959 \longrightarrow 10.0$ Total
0.1

.2

2

3 
.. ESTIMATE OF Sr 90 DEPOSITION FROM GUMMED FILM

STATION \#_ 824

LOCATION DACCA, PAKISTAN

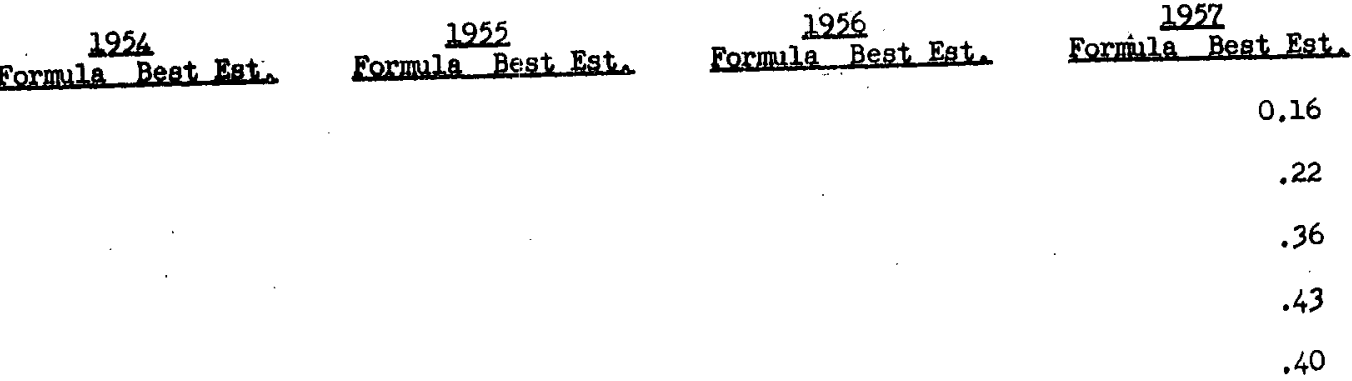

May

June

July

Aug.

Sept.

Oct.

Nov.

Deo.

TOTAL

\section{8}

Formuli Begt Est:

Jen.

0.66

1959

Feb.

.33 .

March

.36

April

.90

.72

May

.35

June

.35

July

Aug.

Sept.

oct.

.21

.25

.08

.08

Formula Begt Est.

0.33

Nov.

.03

.82

1.32

.73

1.27

.78

.22

.42

.06

.18

Pre-1954

1954

1955

1956

1957

$1958 \quad 4.03$

$1959 \quad 6.23$

rotal

Dec.

TOTAL

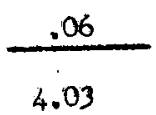

.03

.07

6.23 
ESTIMATE OF INFINITY $\gamma$ DOSE FROM GUMMED FILM

STATION \#- 824

LOGATION DACCA, PAKISTAN

1954

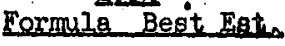

1955

Eomula Best Bato 1956 Dormula Begt Est.

Jan.

Dob.

Maroh

Apr11

May

June

JuIy

Aug .

Sept.

Oct.

Nov.

Dec.

TOTAL

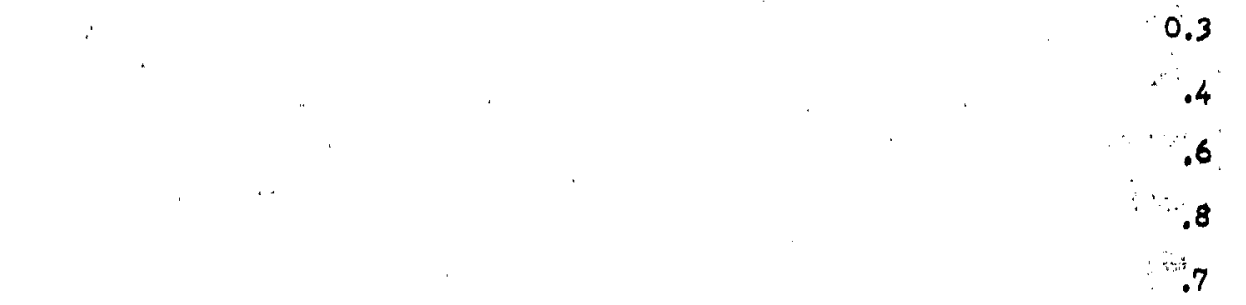

Dec.
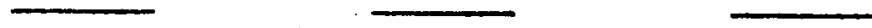
ESTRMATE OP Si 90 DEPOSITION FROM GUMMED FIIM

STATION: \#

LOCATION_BANGKOK, THAILAND

. 2954

Eormula Best Est.

Jen.

Feb.

March

April

May

June

July

Aug.

Sept.

Oot.

Nov.

Dec.

TOTAL
0.01

.05

.37

.08

.33

.10

.10

.07

.02

.01

.05

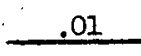

1.20
1955.1956

Formule Best. Est.

$0.01 *$

$.01 *$

$.02 *$

$.02 *$

$.03 *$

$.04 *$

$.04 *$

$.02 *$

$.04 *$

$.06 *$

Formule Best Est.

$0.06 *$

$.05 *$

.08

$.11 *$

.06

.01

.06

.36

.32

.01

.18

.01

.02

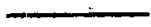

\begin{tabular}{l} 
Est \\
08 \\
06 \\
01 \\
06 \\
36 \\
32 \\
01 \\
01 \\
02 \\
\hline
\end{tabular}


ESTIMATE OF INFINITY $y$ DOSE FROM GUMMED FILM

STATION \# 825

LOCATION BANGKOK, THAILAND

1954

1955

1956

1957

Formula Best Est. Formula Best Este Formula Best Est. Formula Best Est.

Jan.

0.0

$0.0 *$

$0.1 *$

Feb.

.1

March

1.8

Apr11

.3

May

2.1

June

.2

JuIy

.2

Aug.

.1

Sept.

. .1

$.0 *$

oct.

.1

$.0 *$

$.0 *$

. I*

$.1 *$

$.1 *$

$.0 *$

$.1 *$

$.1 *$

$.1 *$
$.2 *$

.1

Nov.

.2

Dec.

.0

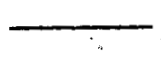

5.2

TOTAL

1958 Formula Best Est.

1259.

Formula Best Est.

Jan.

0.1

.5

Feb.

.0

.5

March

.0

April

.1

May

June

.3

July

1.8

Aug.

.7

Sept.

.2

Oct.

.1

.3

.3

Pre-1954

1.0

1954

1955

.3

1956

.3

1957

.2

1958

3.9

1.0

1959

4.7

Nov.

.1

.1

Total

Dec.

1

.1

TOTAL

3.9

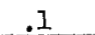

4.7 
STATION \#_ 826

LOCATION GHIENGMAI, THAILAND

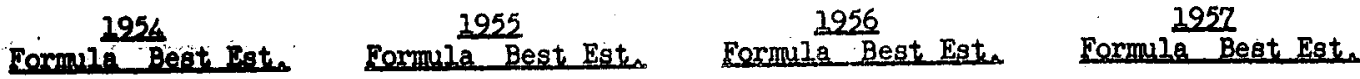

Jen.

Feb.

Maroh

April

.16

May

.23

June

.14

JuIy

.22

Aug .

.12

Sept.

.04

oot.

.05

Nov.

.01

Deo.

.06

TOTAL

\subsection{9}

Formize Beat Eat. Eermila Best Est.

Jan.

0.05

0.11

Feb.

.07

.53

March

.01

.42

April

.10

.81

May

.15

June

.09

July

.10

Aug.

.07

.55

.15

.10

.12

Sept.

.12

Oct.

.07

.11

Nov.

.02

.50

Pre-1954

1954

1955

1956

1957

$1958 \quad .84$

1959

3.54

Dec.

TOTAL

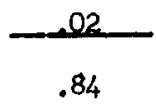

.07

.07

3.54 
STATION \# 826

LOCATION_CHIENGMAI, THAIIAND

2954

Formula Besit Eat.

Jan,

Feb.

March

Apr11.

May

June

July

Aug.

Sept.

Dot.

Nov:

Deo.

TOTAL

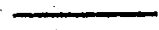

1955

Formula Best Est.

1956

Formula Best Est.
1957

Formula Best Est.

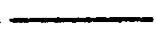

1958

Formula Best Est.

Jan.

Feb.

March

Apri1

May

June

July

Aug.

Sept.

Oct.

Nov.

Dec.

TOTAL

\section{1}

.1

.0

.2

.3

.2

.3

.2

.3

.2

.1

.0

2.0

1959

Formula Best Est.

0.2

I. 1

.8

1.5

Pre-1954

1.0

.3

.2

.2

.2

.8

1954

1955

1956

1957

$1958 \cdot 2.0$

$1959-6.5$ Total

.1

.1

6.5 
DSTMATE OF Sr 90 DEPASTTION FROM GÜMED FIL

STATION $/ 828$

LOCATION SONGKHILA, THAILAND

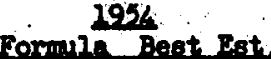

1955

1957

Bormul Best Bst. Eormulo Best Este Eormula Best Est. Eormula Best Est.

Jan.

Feb.

Maroh

.12

April

.04

May

.01

June

.00

JuIy

.15

Aug.

.11

Sept.

.02

Oot.

.16

Nov.

.03

Deo.
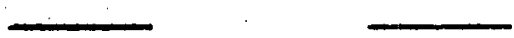

.02

TOTAL

Formula Best Bat. $\frac{1958}{1959}$

\begin{tabular}{lcc} 
Jan. & 0.07 & 0.13 \\
Feb. & .02 & .10 \\
Maroh & .01 & .12 \\
\hline Apr11 & .01 & .07 \\
May & .02 & .21 \\
June & .10 & .17 \\
July & .61 & .14 \\
Aug.: & .16 & .01 \\
Sept. & .16 & .12 \\
Oct. & .04 & .02 \\
Nov. & .13 & .03 \\
Deo. & .18 & .06 \\
Total & 1.51 & 1.18
\end{tabular}



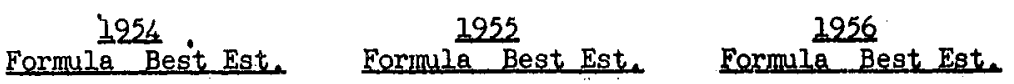

2957

Jan.

Feb.

March

Apre1l

May

June

July

Formula Best Est.

Foxmila Best Est.

Formula Best Est.

Aug.

Sept.

Oot.

Nov.

Deo.

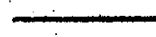

TOTAL

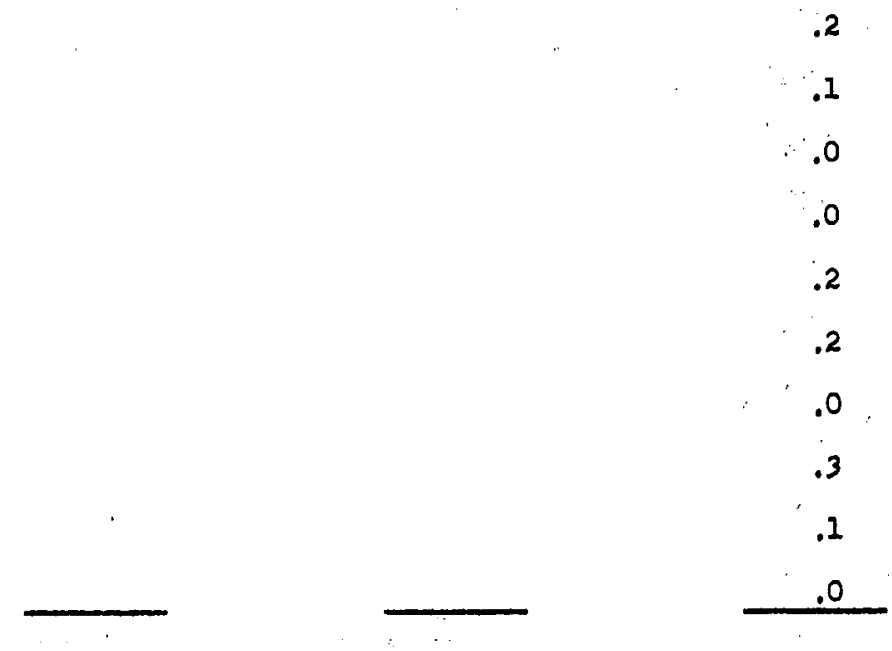

Jan.

Feb 。

March

April

May

June

Ju1y

Aug .

Sept.

Oct.

Nov.

Dec.

TOTAL

\section{1}

.0

.0

.0

.0

.2

1.6

.4

.4

.1

.4

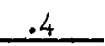

3.6
1959

Formula Best Eat.

0.3

.2

.2

.1

.4

.3

.2

.0

.2

.0

Fre-1954

1954

1955

1956

1957

$1958 \quad 3.6$

1959

2.0

Total 
ESTIMATE OF Sr 90 DEFOSITION FROM GUMMED FILM

STATTON \#. 827

LOCATION_ UBOL, THAIILAND

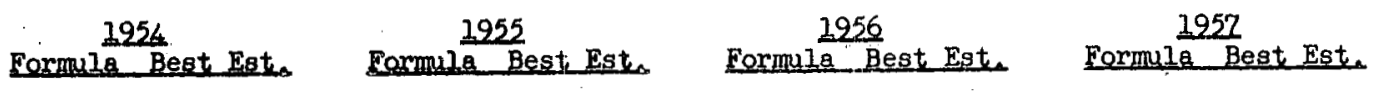

Jan.

Feb.

March

April

.06

May

.01

.

.11

June

.07

JuIy

.27

Aug.

.11

Sept.

.21

oct.

.03

Nov.

.01

Deo.
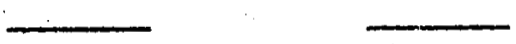

.01

TOTAL

1958

1959

Formula Best Est. Formule Best Est.

Jan.

0.04

0.04

Feb.

.01

.06

March

.04

.14

April

.06

.10

May

.28

June

.18

July

.81

Aug.

.21

Sept.

.24

Oct.

.07

Nov.

.10

Dec.

.01

.16

.51

.11

.14

.46

.06

Pre-1954

1954

1955

1956

1957

$1958 \quad 2.05$

1959

1.90

Total

TOTAL

2.05

.07

.05

1.90 
ESTIMATE OF INFINITY $y$ DOSE FROM GUMMED FILM

STATION \#_827

LOCATION UBOL, THAILAND

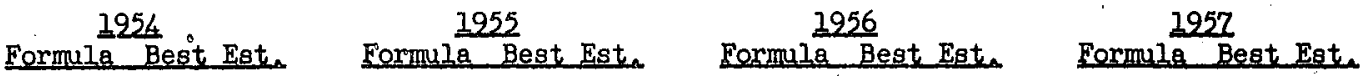

Jan,

Feb.

March

April

May

June

July

Aug .

Sept.

.1

.0

.2

.1

.4

.2

oct.

.3

Nov.

Dec.

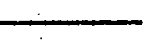

TOTAL

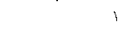

, Jan.

Feb.

March

April

May

June

July

Aug.

Sept.

Oct.

Nov.

Dec。

TOTAL,
0.1

.0

.1

.1

.6

.4

2.2

.5

.6

.2

.3

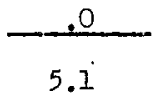

0.1

.1

.3

.2

.3

.9

.2

.2

.7

.1

Pre-1954

1954

1955

1956

1957

$1958 \quad 5.1$

1959

3.3

Total

.1

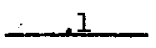

3.3 


\section{4}

Cormula Beat Egt.

Formule Best. Est

1256

1957

Jan.

Feb.

March

April

May

June

JuIy

Aung .

Sept.

Oct.

Nov.

Deo.

TOTAL
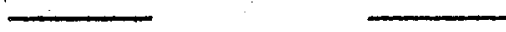

1958

Formula Begt Egt

Jan.

0.20

.28

.11

.09

.80

.30

June

.54

July

Aug.

Sept.

Oct.

Nov.

Dec.

TOTAI
1252

Tormula Best Est.

0.22

.31

.12

.52

Pre-1954

1954

1955

1956

1957

$1958 \quad 2.93$

1959

2.53

.10

Total

.10

10

2.53 


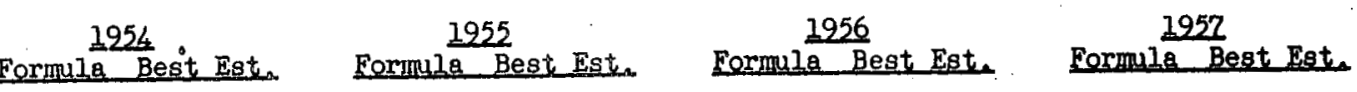

Jan.

Feb.

March

Apr11

May

June

July

Aug.

Sept.

oct.

Nov.

Dec ,

TOTAL

ormula Best Est.

Formula Best Est. 
BSTTMATE OF $\mathrm{Sr}^{90}$ DEPOSITION FROM GUMMUD EILM

STATION

Tormula Beat sot.
LOCATION RANGOON, BURMA

1956

Bormule Best Est.
1952

Formulai Best Est.

Jañ.

Peb.

Maroh

Apr11

Way

June

JuIy

Aug.

.22

Sept.

1.48

Dot.

.10

Nov.

.06

Deo.

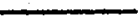

.03

Torar

$1958 \quad 2959$

Povula Best Sot. Eermula Best-Eot.

Jan.

Feb.

Maroh

April

May

June

July

Aus:

Sept.

Oct.

Nov.

Deo.

TOTAL
0.03

.35

.06

1.33

.16

.49

.67

.34

.18

.10

.04
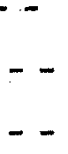

.22

.44

.25

-

.27

$$
\begin{array}{r}
\text { Pre-1954 } \\
1954 \\
1955 \\
1956 \\
1957 \\
1958 \\
1959
\end{array}
$$

Total 

Jan.

Feb.

March

Apr11

May

June

July

Aug.

Sept.

2.5.

Oct.

Nov.

Deo.

TOTAL

1958

Formula Best Est.

0.1

.6

Feb.

March

Apri1

May

June

July

Aug.

Sept.

Oct.

Nov.

Dec.

TOTAL
1959

Formula Best Est.
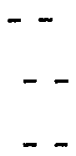

.4

Pre-1954

1954

1955

1956

1957

1958

1959

Total 


\section{ESTIMATE OF ST 90 DEFOSITION FROM GUMMUD FILM}

STATION $\#$

1954.

Eoxmuin Best Est.

1955

1956

1927

Formule Best Este Formula Best Est. Formule Best Est.

Jan.

Teb.

March

Apre1

May

June

JuIy

Aug.

Sept.

oot.

Nov.

Deo.

TOTAL

Pomula Best Est. Eormula Best Eet.

Jan.

Feb.

Maroh

April

May

June

July

Aug.

Sept.

oet.

Nov.

Deo.

TOTAL
0.76

.33

1.28

.95

.54

. .14

.26

.32

.22

.51

Pre-1954

1954

1955

1956

1957

1958

1959

5.91

.35

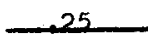

5.91 

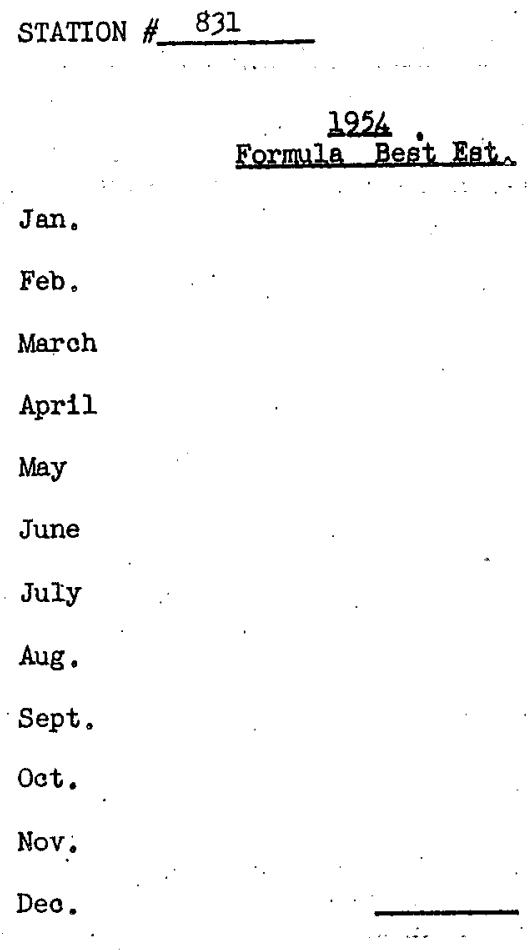

LOCATION TAIPEI, TAIWAN, THIRD STATION

$\therefore$

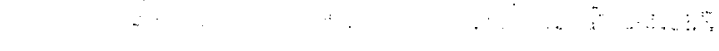

a:

$x$

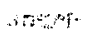

$\therefore \therefore$

tents
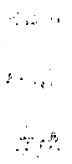

. An

1958

Formula Best Esta

Jen.

Feb。

March

April

May

June

July

Aug.

Sept.

oct.

Nov.

Dec.

TOTAL

$\begin{array}{r}1.3 \\ 1.1 \\ 2.4 \\ .4 \\ .4 \\ .3 \\ .3 \\ .6 \\ \hline\end{array}$

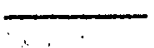

1959

Formula Best Est.

$$
1.6
$$

.7

2.4

1.7

Pre-1954

1954

1955

1956

1957

1958

1959

10.4

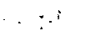

s
.9

.2

.4

.5

.3

.8

.5

.4

10.4 
ESTIMATE OF $\mathrm{Sr}^{90}$ DEPOSITTON TROM GUMMDD FIIM

STATION \#- 832

LOCATION AKYAB, BURMA

1954

2957

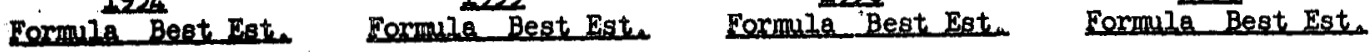

Jan.

Jeb.

Maroh

April

May

June

JuIy

Aug.

.08

Sept.

.13

oot.

.03

Nov.

.05

Deo.
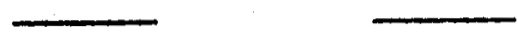

.01

TOTAL

1958

Formula Best EBt. Formula Best Est.

Jan.

0.01

Feb.

Varch

April

.33

Nay

.39

June

.38

July

.63

Aug.

.36

Sept.

.20

oct.

.00

Pre-1954

1954

1955

1956

1957

1958

1959

Nor.

.00

Dec.

.00

TOTAL 
STATION \# 832

LOCATION AKYAB, BURMA

1954

1955

Formula Best Est Formule Best Est.
1956

Formule Best Est.
1957

Formula Begt Esta:

Jan。

Feb.

March

Apr11

May

June

July

Aug.

Sept.

Oct.

Nov.

Deo.

TOTAL

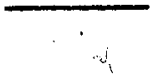

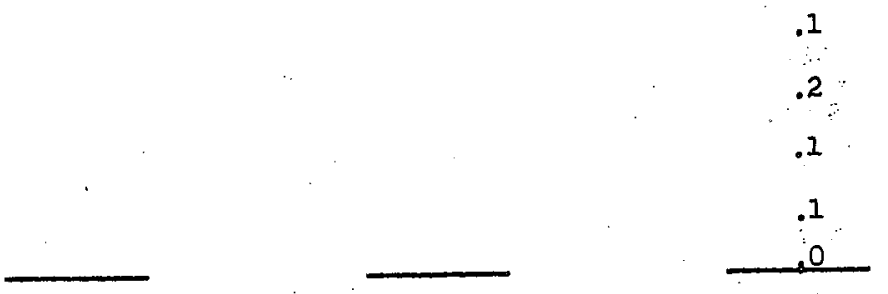

1959

Formula Best Esta Formula Best Est.

Jan.

0.0

Feb.

March

Apri1

May

.6

.8

.8

June

1.6

July

1.0

.5

Sept.

Oct.

.0

Pre-1954

1954

1955

1956

1957

1958

1959

Total

Nov.

.0

Dec.

.0

TOTAL 


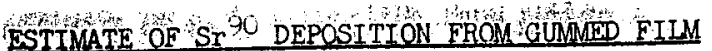

STATTON 834

LOCATION MOULMETN, BURMA

Formula Best Eate Eormula Best Este Formula Best Est. Formula Best Est.

Jan.

seb.

March

Apri1

May

June

JuIy

Aug.

Sept.

Dot.

Nov.

Deo.

ioras.

2958

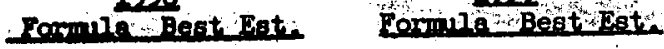

Jon.

0.11

reb.

Maroh

April

May

.24

June

.56

July

.63

Autg:

.21

Sept.

.21

Dot.

.10

1954

1955

1956

1957

1958

1959

Total

Nov.

Dec.

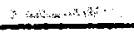

rotax: 
STATION \# 834 LOCATION_ MOULMEIN, BURMA

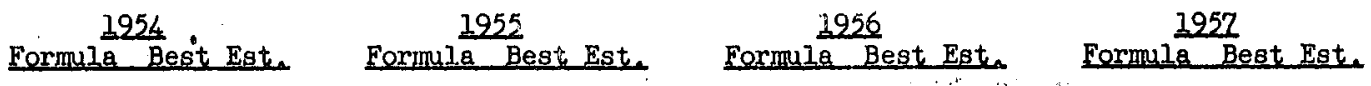

Jan。

Feb.

March

April

May

June

July

Aug.

Sept.

Oct.

Nov.

.1

Dec.

TOTAL

Formula Best Est. Formula Best Est.

Jen.

0.2

Feb.

March

Apr11

May

June

July

Aug .

Sept.

Oct.

1.7

.5

.5

.3

Pre-1954

1954

1955

1956

1957

1958

1959

Total

Nov.

Dec.

TOTAL 


\section{ESTIMATE OF Sir 90 DEPOSITION FROM GUMAUD FILM}

STATION \#-833

1954

Eormula Best Est.
LOCATION LASHIO, BURMA

Cormula Bestestio Formula Best Est. Formula Best Est.

Jan.

Feb.

Maroh

Apr11

May

June

Juily

Avg.

Sept.

.33

Oot.

Nov.

.04

Deo.
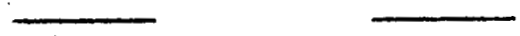

.10

TOTAL

Formule Best Est. Formula Best Est.

Jan.

0.06

Feb.

.11

March

.25

April

.29

Pre-1954

May

.80

1954

June

.23

1955

1956

July

.24

1957

Aug.

.13

1958

Sept.

1959

Oct.

Total

Nov.

Dec.

TOTAL 
ESTIMATE OF INFINITY y DOSE FROM GUMMED FILM

STATION \# 833

LOCATION_IASHTO,_BUTRA

\section{4}

Formula Best Est.

1955

Formula Begte Est.
2956

Eormula Best Est.
1957

Eormula Best Est.

Jan。

Feb.

March

April

May

June

JuIy

Aug.

Sept.

Oct.

Nov:

Dec.

TOTAL
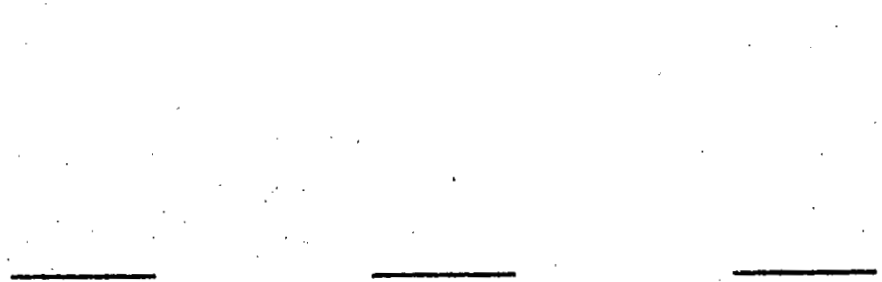

.5

.5

.1

.1

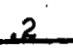

1958

Formula Best Est.

0.1

.2

Feb.

March

Apri1

May

June

July

Aug.

Sept.

Oct.

.5

.5

1.6

.5

.6

.3

Nov.

Dec.

TOTAL
1959

\section{Eormula Best Est.}

Pre-1954

1954

1955

1956

1957

1958

1959

Total 
STATION \# 835

2956

Eormula Best Eat.

Jan.

Feb.

March

April

May

June

JuIy

Aug.

Sept.

oct.

Nov.

Deo.

TOTAL
LOCATION PROME, BURMA
1955

Formule Best Esto Formule Best Est.
2957

Formule Best Est.

\section{8} 1959

Formule Best Est. Eormiti Best Estia

Jan.

.10

Feb.

.16

Maroh

.22

April

.15

May

.07

0.02

.31

.08

.05

June

.25

1956

July

.39

1957

Aug :

1958

Sept.

1959

Oct.

Total

Nor.

Dec. TOTAI 
ESTIMATE OF INFINITY $y$ DOSE FROM GUMMED FILM

STATION \# 835

LOCATION_PROME, BURMA

\section{4}

Formula Best Est. Formula Best Est.

Eormule Best Esto

Jen.

Feb。

March

April

May

June

JuIy

Aug.

Sept.

Oct.

Nov.

Dec.

TOTAL

1958

Formula Best Est. Formula Best Est.

Jan.

0.2

Feb.

.3

March

.4

April

.3

May

.1

June

.5

July

1.0

1959

Aug.

Sept.

Oct.

Pre-1954

1954

1955

1956

1957

1958

1959

Total

Nov.

Dec.

TOTAL 
ESTIMATE OF Sr 90 DEPOSITION FROM GUMMED EIIM

STATTON \# 837

LOCATION_MANDALAY, BURMA

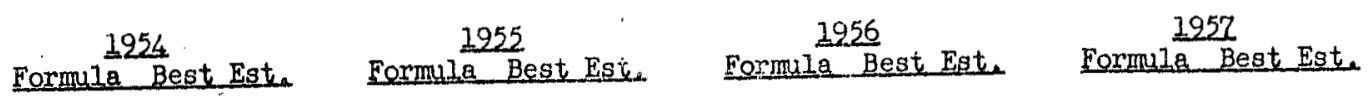

Jen.

Feb.

March

April

May

June

July

Aug.

Sept.

Oct.

Nov.

Dec.
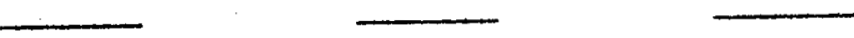

TO'IAL

1958

1952

Formula Best Este Formula Best Est.

Jan.

0.02

0.06

Feb.

.01

.08

March

.29

.26

Apri1

.28

-

May

.36

.10

.16

.18

.21

.11

.05

Sept.

.18

Pre-1954

June

.13

Oct.

.08

Nov.

.09

Dec.

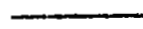

TOTAL 


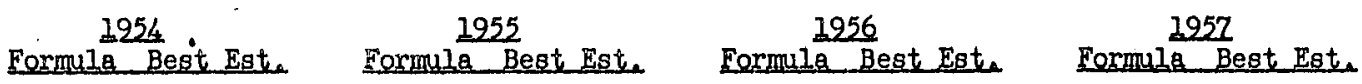

Jan.

Feb.

March

Apr11

May

June

Juiy

Aug 。

.1

Sept.

Oct.

Nov:

Dec.

TOTAL

1958

Formle Begt Eet. Formula Begt Eet.

Jan。

0.0

0.1

Feb。

.0

.2

March

.6

Aprt1

.5

.5

$-$

Pre-1954

May

.7

.2

1954

June

.3

.3

1955

1956

Ju1y

.4

.3

1957

Aug.

.2

1958

Sept.

.1

1959

oct.

.3

Total

Nov.

.1

Dec.

$-$

.1

TOTAL 


\section{ESTIMATE OF Sr 90 DEPOSITIOR FROM GUMMAOP FIM}

STATION \#_ 838

LOCATTON MYITKYINA, BURMA

Formula Best Est. Formula Best Este Formula Best Est. Formule Best Est.

Jan.

Feb.

March

ApriI

May

June

July

Aug.

Sept.

oct.

Nov.

Deo.

TOTAL
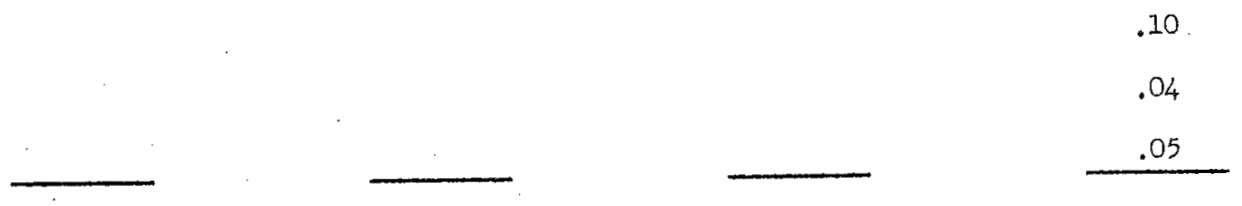

Foxmula Best Est. Formula Best Est.

Jan.

0.12

Feb.

.24

March

.65

Apr11

.39

May

.27

June

.24

July

.08

Pre-1954

1954

1955

1956

Aug.

.20

1957

1958

Sept.

1959

oct.

Total

Nov.

Dec .

TOTAL 
1254 Formula Best Est.
1955

Formule Best Est.
1956

Tarmule Best Est.

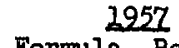

Formuli Best Est.

Jen.

Feb.

March

April

May

June

JuIy

Aug.

Sept.

Oct.

Nov.

Deo.

TOTAL

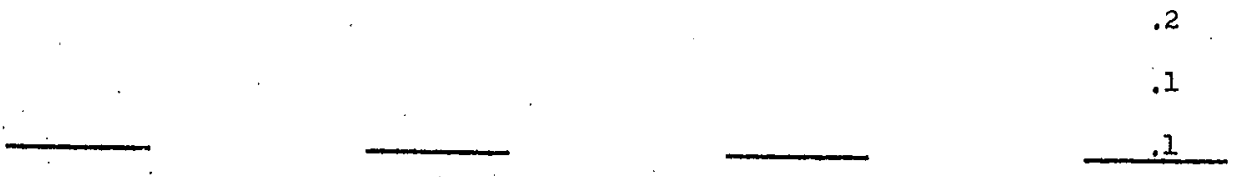

Formula Best Este Formula Best Est.

'Jan.

Feb.

March

Apr11

May

June

July

Aug .

Sept.

Oct.

Nov.

Dec.

TOTAL
0.2

.4

1.2

.8

.6

.6

.2

.5

Pre-1954

1954

1955

1956

1957.

1958

1959

Total 
ESTIMATE OF Sr 90 DEPOSTITON FROM GUMMED EILM

STAITION \# 836

IOCATTON MERGUI, BURMA

1954

Formila Best Est.

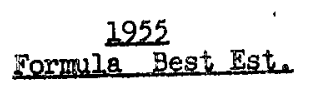

$\frac{1256}{8 e}$

1957

Eormula Best Est. Formula Best Est.

Jan.

Feb.

March

Apr1I

May

June

JuIy

Aug.

Sept.

.24

Oct.

.41

Nov.

Dec.

TOTAL

Formula Best Eate Formula Best Est.

Jen.

0.11

Feb.

March

Apr11

.07

May

.14

June

.14

July

.34

Aug.

.17

Sept.

.28

Oct.

.18

Pre-1954

$1954^{\prime}$

1955

1956

1957

1958

1959

Total

Nov.

Dec.

TOTAL 
STATION \# 836

Formula Best Est. Eormula Best Est.
LOCATION_ MERGUI, BURMA.

1956

Formula Best Est.
2957

Formila Begt Est.

Jen.

Feb.

March

Apr11

May

June

JuIy

Aug.

Sept.

Oct.

Nov

Dec.

TOTAL

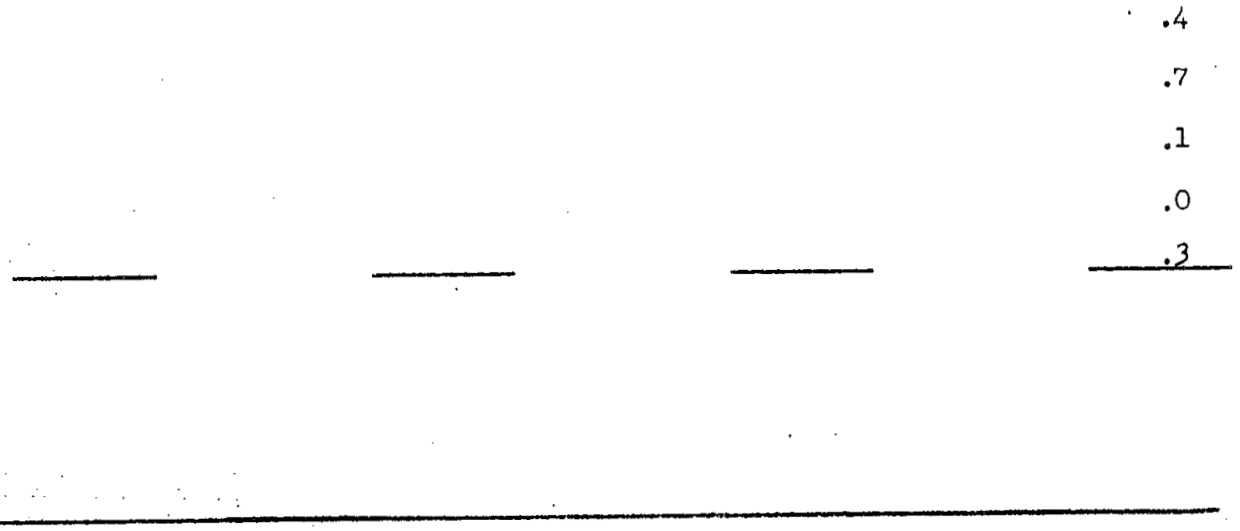

1958

1259

Formula Best Est. Formula Best Esta

Jan,

0.2

Feb.

.0

March

Apr11

May

.1

Pre-1954

June

.3

.3

July

.9

1954

1955

1956

1957

Aug.

.5

1958

Sept.

.6

oct.

.5

1959

Total

Nov.

Dec .

TOTAL 
ESTIMATE OF Sr 90 DEPOSITION FROM GUMMED FIIM

STATION \#-901

LOCATION GUAM, MARIANAS

1254

Formula Best Egt.

1 B Best Est.

Jan.

.04

Feb. .09

March

$1.43 \quad 0.43$

Apr11.

.48

May

1.54

June

.28

JuIy

.16

Aug.

.20

Sept.

.16

Det.

.19

Nov.

.17

Dec.

TOTAL

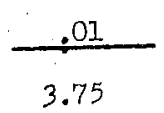

3.75

$0.04 *$

$.05 *$

$.09 *$

$.08^{*}$

$.28 *$

$.07 *$

$.05 *$

$.05 *$

$.08 *$

$.13 *$

.14

$.19 * *$

1.25

1956 Dormula Best Est.

$0.78 *$

1957

\begin{tabular}{ccc} 
& $0.78 *$ & 0.25 \\
& .30 & .30 \\
& $.65 *$ & .24 \\
& $.23 *$ & .21 \\
10.57 & 4.09 & .29 \\
8.30 & 3.16 & .15 \\
2.61 & 1.52 & .20 \\
& 1.42 & .27 \\
& .64 & .23 \\
& .53 & .30 \\
& .30 & .07 \\
& .33 & .29 \\
\hline 13.95 & 2.80
\end{tabular}

Formula Best Esta Formula Best Este

$\begin{array}{lccc}\text { Jan. } & .14 & 0.48 \\ \text { Feb. } & .23 & .75 \\ \text { March } & .19 & .87 \\ \text { Apr11 } & .22 & .71 \\ \text { May } & 5.93 & 5.18 & .49 \\ \text { June } & 8.60 & 5.67 & .17 \\ \text { July } & 12.65 & 5.81 & .30 \\ \text { Aug. } & & 1.13 & .26 \\ \text { Sept. } & & .45 & .18 \\ \text { Oct. } & & .18 & \\ \text { Nov. } & & .23 & \\ \text { Dec. } & & .24 & \\ \text { TOTAL } & & 19.67 & \end{array}$


STATION \#_901

\begin{tabular}{|c|c|c|}
\hline & \multicolumn{2}{|c|}{ Formula Best Esto } \\
\hline $\operatorname{Jan}$. & & 0.0 \\
\hline Feb. & & .2 \\
\hline March & 4.2 & 2.7 \\
\hline Aprtl & & 2.7 \\
\hline Mey & & 8.2 \\
\hline June & . & 1.0 \\
\hline JuIy & & .5 \\
\hline Aug. & & .6 \\
\hline Sept. & & .4 \\
\hline Oct. & & .5 \\
\hline Nov. & & .4 \\
\hline Dec. & & .0 \\
\hline TOTAL & & 17.2 \\
\hline
\end{tabular}

LOCATION GUAM, MARIANAS

\section{5 Formulis Best Est.}

$0.1 *$

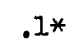

$.2 *$

$.2 *$

$.5 *$

$.1 *$

$.1 *$

$.1 *$

$.1 *$

$.2 *$

.2

$.3 *$

2.2
1956 Formule Best Est.

$1.3 *$

.5

1.1*

.3*

$21.5 \quad 7.1$

16.14 .5

7.1

2.7

3.3

1.4

1.0

.6

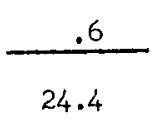

1952 Formula Begt Est.

0.4

.5

.4

.4

.5

.3

.3

.4

.4

.8

.1

.5

5.0

\begin{tabular}{l}
.4 \\
.5 \\
.4 \\
.4 \\
.5 \\
.3 \\
.3 \\
.4 \\
.4 \\
.8 \\
.1 \\
.5 \\
\hline .0
\end{tabular}


ESTIMATE OF Sr 90 DEPOSITION FROM GUMMEO EILM

STATION H. 903

LOCATION IWO JIMA, VOLCANO ISIANDS

Formila Best Est. Formula Best Est. Eormig Best Est. Formula Best Est.

Jan.

Feb.

March

April

May

June

JuIy

Aug .

Sept.

Oct.

Nov.

Deo.

TOTAL
.04

.04

.04

.12

.31

.15

.11

.08

.09

.10

.15

.05

1.28
$0.11 *$

.11

$.08 *$

$.19 *$

$.08 *$

$.06 *$

$.07 *$

$.05 *$

$.07 *$

$.14 *$

.37

.24

1.57
$0.28 *$

.17

$.25 *$

$.14 *$

.32

.10

.18

.34

.23

.14

.11

.40

2.66
0.37

.49

.52

.94

.26

.06

.18

.08

.17

.13

.25

.23

3.68

Formula Best Est. Formuls Best_Est.

Jan.

0.11

0.65

Feb.

.35

.27

March

.41

.58

Apri1

.30

May

.77

.65

.30

June

2.01

July

3.42

2.04

.18

Aug.

.26

.22

Sept.

.14

oct.

.23

Nov.

.14

Dec.

TOTAL

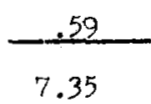

.19

.14

.40

.17

.25

3.90 
STATION \#_ 903
LOCATION IWO JIMA, VOLCANO ISIAANS

1957

Formula Best Est. Formula Best Est. Eormula Best Est. Formula Best Est.

$\begin{array}{lcc}\text { Jan. } & 0.0 & 0.2 * \\ \text { Feb. } & .1 & .2 \\ \text { March } & .2 & .2 * \\ \text { April } & .7 & .3 * \\ \text { May } & 1.5 & .1 * \\ \text { June } & .6 & .1 * \\ \text { JuIy } & .4 & .1 * \\ \text { Aug. } & .2 & .1 * \\ \text { Sept. } & .2 & .1 * \\ \text { Oot. } & .2 & .2 * \\ \text { Nov: } & .4 & .6 \\ \text { Dec. } & .1 & .4 \\ \text { TOTAL } & 4.6 & 2.6\end{array}$

$0.5 *$

.3

$.4 *$

$.2 *$

.7

.2

.4

.7

.5

.3

.2

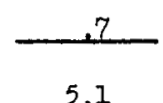

5.1
0.7

.8

.8

1.6

.4

.1

.3

.1

.3

.3

.5

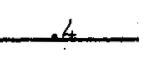

6.3
2958

Formule Best Egt.

Jan.

Feb.

March

April

May

June

July

Aug.

Sept.

Oct.

Nov.

Dec.

TOTAL
0.2

.6

.8

.5

1.5

4.1

$9.3 \quad 4.3$

.7

.3

.7

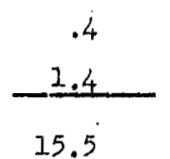

1959

Formla Best Eat.

1.4

.5

1.1

1.2

.5

.3

.4

.3

.2

.6

\begin{tabular}{rr} 
Pre-1954 & 91.5 \\
1954 & 4.6 \\
1955 & 2.6 \\
1956 & 5.1 \\
1957 & 6.3 \\
1958 & 15.5 \\
1959 & 6.9 \\
\hline
\end{tabular}

Total

1.32 
ESTIMATE OF Sr 90 DEPOSTITON FROM GUMMED FILM

STATTON \#- 905

LOCATION_JOHNSON ISIAND

2254

12 Best Est.

$0.08 *$

.11

$.09 *$

.II*

$.08 *$

$.08 *$

$.04 *$

$.05 *$

$.05 *$

$.06 *$

.33

Nov.

Deo.

TOTAL
3.59

.14

.05

.08

.20

.08

3.07

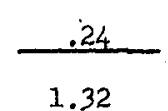

1.32

2956 Formuli Best Est. Formula Best Est.

$0.66 *$

0.29

.49

.29

$.76 *$

.29

$.46 *$

.18

.52

.25

.92

.15

.49

$.43 * *$

.37

.12

.21

.12

.18

.07

.32

$\frac{.16}{2.84}$

7.47

2.84

1259

Formula Best Est. Formula Best Este

Jan.

0.21

.54

Feb.

.21

.85

March

$1.16 * *$

.78

Apri1

.83

.55

May

.46

.44

June

.55

.21

July

1.64

.11

Aug.

.35

.13

Sept.

0.21

.12

oct.

.21

.06

Nov.

.26

.14

Dec.

TOTAL

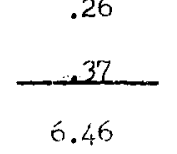

.14

4.07 
STATION \#_ 905

LOCATION

JOHNSON ISLAND

1954 Formula Besit Est.

Jan.

Feb.

March

Apr11

May

June

JuIy

Aug .

Sept.

Oet.

Nov.

Deo.

TOTAL
0.0

.1

$7.7 \quad 1.4$

$23.8 \quad 7.5$

4.5

.5

.2

.2

.1

.2

.4

.1

15.2
1955 Formule Best Est. $0.2 *$

.2

$.2 *$

$.2 *$

$.1 *$

$.1 *$

$.1 *$

$.1 *$

$.1 *$

$.1 *$

.5

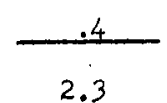

2.3

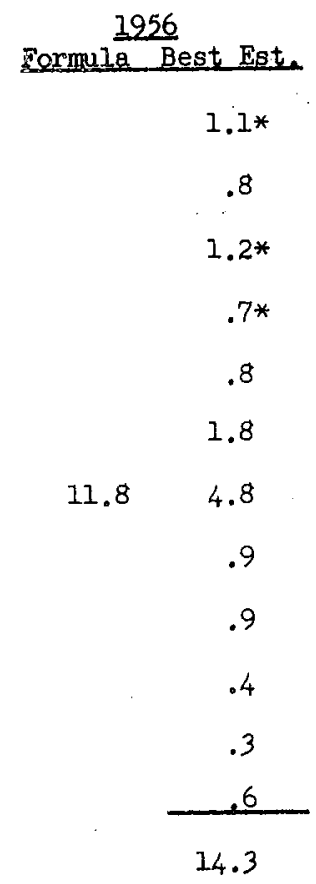

Formule Best Est.

0.5

.5

.5

.3

.4

.3

.8

$.7 * *$

.2

.3

.1

14.3

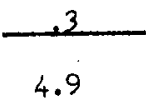

4.9

0.3

Feb.

March

Apr11

May

June

July

Aug .

Sept.

Oct.

Nov.

Dec.

TOTAL
.3

$2.3 * *$

1.4

.9

1.1

4.2

.9

0.5

.6

.7

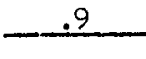

14.1
Formule Best Eat.

1.1

1.7

1.5

1.0

.8

.4

.2

.2

.2

.1

.2

.2

7.6

\begin{tabular}{rr} 
Pre-1954 & 2.2 \\
1954 & 15.2 \\
1955 & 2.3 \\
1956 & 14.3 \\
1957 & 4.9 \\
1958 & 14.1 \\
1959 & 7.6 \\
\hline
\end{tabular}

Total

61 


\section{ESTIMATE OF Sr 90 UEPOSITION FROM GUMAD EILM}

STATION \# 904

LOCATYON_MANIIA PHILLIPINES, CLARKE AFB

1254 Eormula Best Est.

Jan.

0.04

1955

1952

i'eh.

.04

Formula Best Est. Tormula Rest Est.

Formula Best Est.

March

.30

Apri1

.26

May

.33

June

.23

July

.20

Aug .

.08

Sept.

.25

Oct.

.07

$0.03 *$

$0.12 *$

0.05

Nov。

.04

Dec.

TOTAL

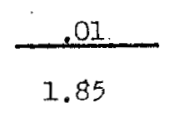

.04

$.00 *$

.02

.03

$.17 *$

$.01 *$

$.07 *$

$.04 *$

$.03 *$

$.05 *$

$.13 *$

.08

$.09 *$

.05

$.08 *$

.10

$.58 * *$

.15

.78

.07

$2.33 \quad 0.64$

.23

.20

.40

.14

.16

.09

.14

.03

.11

.21

.06

3.18

1.35

1958

Formula Best Este Formula Best Est.

Jan.

0.09

.07

Feb.

.20

.06

March

.08

Apr11

.20

.18

May

.84

.12

June

.55

July

.91

Aug.

.25

Sept.

.14

.12

.12

.10

.12

.17

oct.

.07

.13

\begin{tabular}{rr} 
Pre-1954 & 2.54 \\
1954 & 1.85 \\
1955 & .68 \\
1956 & 3.18 \\
1957 & 1.35 \\
1958 & 3.44 \\
1959 & \\
\hline
\end{tabular}

Total.

Nov.

.3

Dec.

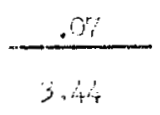

TOTAL

$3, x^{2}$ 
STATION \#_904 LOCATION MANILA PHILLIPINES, CLARKE AF'B

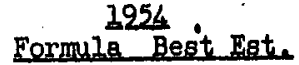

0.0

Jan.

Feb.

March

Apri1

May

June

July

Aug.

Sept.

Oct.

Nov.

Dec.

TOTAL
.1

1.7

1.5

1.7

.9

.7

.1

.6

.2

.1

.0

7.6
1955 Cormule Best Esto

$0.1 *$

.1

$.0 *$

$.3 *$

.0*

$.1 *$

$.1 *$

$.0 *$

.1*

$.1 *$

.1

.1

1.1
1956

Formula Best Eat.

$0.2 *$

.0

.1*

.1*

$.9 * *$

1.5

6.9

1.6

.9

.4

.3

.2

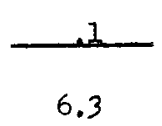

1957

Formula Best Est.

0.1

.1

.1

.2

.3

.1

.4

.3

.2

.2

.1

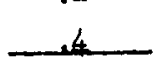

2.5

1958

Formula Best Est.

1959

Formula Best Est.

Jan.

0.1

.2

Feb.

.3

March

April

.2

.1

May

.3

June

1.6

Ju1y

1.2

Aug.

2.5

Sept.

.6

Oct.

.3

.2

.3

.2

.2

.2

.2

.2

.3

Nov.

.1

Dec.

TOTAL,

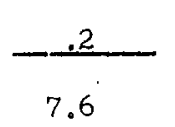

$\begin{array}{rr}\text { Pre-1954 } & 13.4 \\ 1954 & 7.6 \\ 1955 & 1.1 \\ 1956 & 6.3 \\ 1957 & 2.5 \\ 1958 & 7.6 \\ 1959 & \end{array}$

Total 


\section{ESTTMATE OF SY 90 DEPOSITION FROM GUMMET EIIM}

STATION \# 906

LOCATTON FRENCH ERTGATE SHOALS, HAWAIT

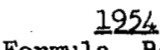

1955

1957

Formula Best Esto Formula BestEst. Formula Best Est. Formula Best

Jan.

0.01

$0.0 / 4 *$

$0.29 *$

0.43

Feb.

.04

$.05 *$

March

.02

April

0.39

$.17 *$

1.21

.37

May

.37

June

.09

$.07 *$

$.52 *$

.21

JuIy

.06

Aug.

.09

Sept.

.06

$.20 *$

$.46 *$

.44

oet.

.20

$.07 *$

.30

.33

Nov.

.10

Dec.

.04

$.06 *$

.45

.19

TOTAL

1.47

$.08 *$

$.07 *$

$.136 *$

.24

$.19 * *$

1.82

1.03

.26

.21

.23

.26

.42

$.20 * *$

1.10

.38

.17

.21

.17

3.24

Formula Best Est

1959

Formula Best Est.

Jan.

0.46

0.26

1.14

Feb.

.33

.74

March

1.09

.51

Apre11

.79

1.01

May

.20

June

.42

July

.26

.73

.38

Aug.

.18

.22

Sept.

.87

oct.

.16

.13

5.63

\begin{tabular}{l}
43 \\
37 \\
21 \\
44 \\
33 \\
.19 \\
26 \\
21 \\
26 \\
$20 * *$ \\
17 \\
17 \\
\hline 24
\end{tabular}

Nov.

Dec.

TOTAL

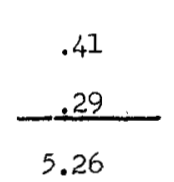

.13

.24

.07

Pre-1954 0.83

$1954 \quad 1.47$

$1955 \quad 1.10$

$1956 \quad 5.63$

$1957 \quad 3.24$

$1958 \quad 5.26$

$1959-5.31$

Total 22.8 
STATION \# 906

LOCAmTON FRENCH FRIGATE SHOAIS, HAWAII

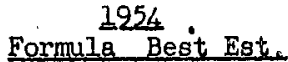

Jan.

Feb.

March

Apr11

May

June

JuIy

Aug.

Sept.

Oct.

Nov.

Dec.

TOTAL
0.0

.0

.1

2.7

2.1

.3

.2

.3

.1

.5

.2

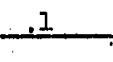

6.6
1955

Cormula Best Est.

$0.1 *$

$.1 *$

$.3 *$

$.1 *$

$.2 *$

$.1 *$

$.1 *$

$.1 *$

$.1 *$

. I*

.2

$.3 * *$

1.8
1956

Formula Best Est.

$0.5 *$

2.0

$.8 *$

$.7 *$

.5

.9

3.7

2.0

.5

.9

.7

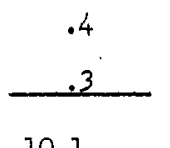

10.1
1957

Formuli Begt Est.

0.7

.6

.3

.8

.6

.4

.4

.3

.5

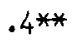

.4

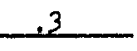

5.7

1958

Formula Best Est. Formula Best Est.

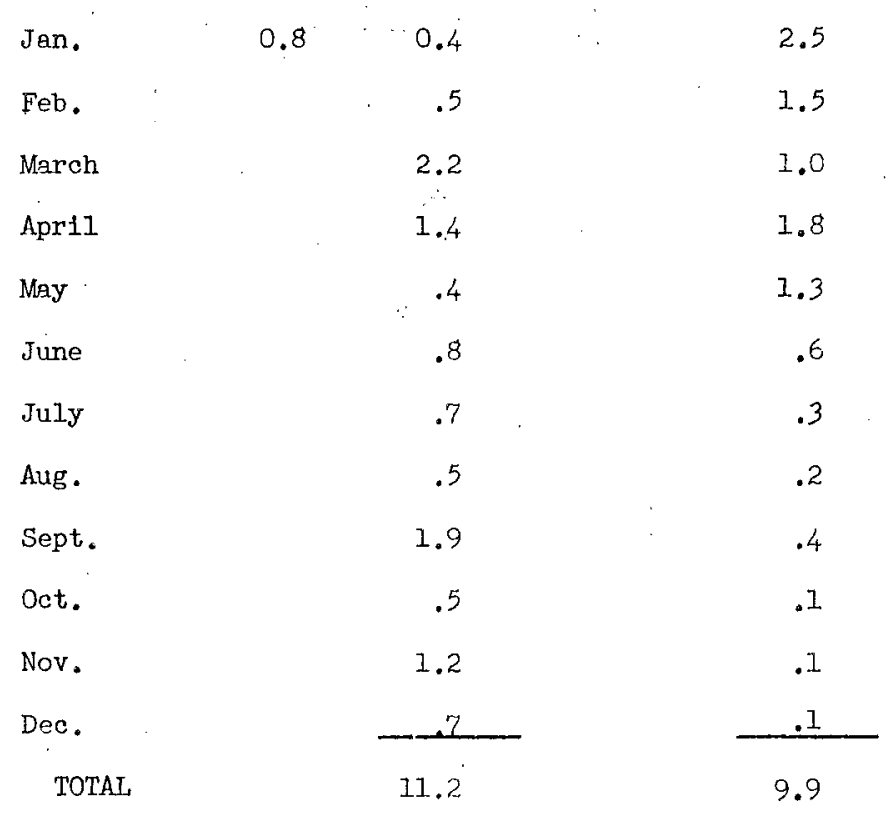

\begin{tabular}{rc} 
Pre-1954 & 3.1 \\
1954 & 6.6 \\
1955 & 1.8 \\
1956 & 10.1 \\
1957 & 5.7 \\
1958 & 11.2 \\
1959 & 9.9 \\
\hline
\end{tabular}


1954 Eormule Best Est.

\begin{tabular}{ll} 
Jan. & 0.01 \\
Feb. & .04 \\
March & .04 \\
April & .03 \\
MAay & .09 \\
June & .07 \\
JuIy & .12 \\
Aug. & .03 \\
Sept. & .12 \\
Oct. & .05 \\
Nov. & .01 \\
Dec. & .04 \\
\hline ToTAL & .65
\end{tabular}

\begin{tabular}{c}
$\frac{1955}{\text { Eormulest Est }}$ \\
$0.07 *$ \\
.52 \\
$.09 *$ \\
$.06 *$ \\
$.07 *$ \\
$.06 *$ \\
$.14 *$ \\
$.12 *$ \\
$.09 *$ \\
$.13 *$ \\
.38 \\
2.36 \\
\hline .09
\end{tabular}

1256 Rormila Best Est.

$0.97 *$

.44

$.20 *$

$.25 *$

.29

.17

.30

.31

.65

.21

.08

$\frac{.26}{4.13}$

1952

Formilia Best Est.

0.60

.46

.30

.72

.54

.14

.29

.39

.20

.19

.09

.13

4.05

1959

Formula Best Est. Formula Best Est.

Jan.

0.49

2.30

Feb .

.58

.76

March

.76

1.72

April

.50

2.60

May

.37

.78

June

$1.94 \quad 1.30$

.65

July

.22

Aug .

.39

Sépt.

.13

oet.

.19

Nov.

.38

Déc.

TOTAL

.74

6.05

.57

.34

.31

.32

.33

.20

10.78 
STATION \# 907

LOCATION_ MIDWAY ISLAND

Formula Besit Est

Jan.

Feb.

March

Apr11

May

June

July

Aug .

Sept.

Oct.

Nov.

Dec.

TOTAL
0.0

.1

.2

.2

.5

.3

.4

.1

.3

.1

.1

.1

2.4
1955

Formule Best Est.

$0.1 *$

1.0

$.2 *$

$.1 *$

$.1 *$

$.1 *$

$.2 *$

$.2 *$

$.1 *$

.2*

.6

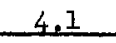

7.0
1956

Eormula Best Est. Eormila Best Est.

$.6 *$

.7

$.3 *$

. 8*

.5

.3

.7

.7

1.4

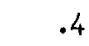

.2

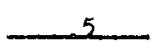

8.1

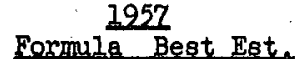

1.0

.8

.5

1.4

.9

.3

.5

.6

.4

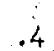

.2

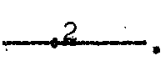

7.2

1958

1952

Formula Best Est. Formula Best Est.

Jan.

0.8

5.0

Feb.

1.0

1.5

March

1.5

Apri1

.9

3.3

4.8

May

.7

June

3.8

2.6

July

.6

Aug.

1.1

Sept.

.3

Oct.

.6

1.3

1.1

Nov.

1.1

Dec.

1.8

TOTAL

13.0

.9

.5

.5

.5

.5

$\frac{.1}{20.0}$

\begin{tabular}{rr} 
Pre-1954 & 5.1 \\
1954 & 2.4 \\
1955 & 7.0 \\
1956 & 8.1 \\
1957 & 7.2 \\
1958 & 13.0 \\
1959 & 20.0 \\
\hline Total & 63.0
\end{tabular}




\section{ESTTMATA OESSY 90 DEPOSTMUN FROM GUMWD FIL}

STATIONN \#- 909

LOCATTON CAVION, PHOENIX ISLALNS

$\because \cdots$

Jan.

Feb.

Mareh

Aprotis

May

June

Juiy

Aug .

Sept.

oct.

Nov.

Dec.

TOTAL
1254. Formula Best Est.

0.04

3.67

. .08

.91

.088

.35

.15

.07

.07

.06

.06

.03

.05

1.95
3955

\section{Formule Best Lis.}

$0.02 *$

.02

$.02 \%$

$.01 *$

$.00 *$

. $01 *$

$.02 *$

$.02 *$

$.01 *$

$.00 *$

.08

.02

.24
1256 Eormula Best Est.

$0.07 *$

.01

$.04 *$

$.0 \div *$

.09

.19

.15

.06

.12

.03

.02

.04

.83
2957

Formula Best Est.

0.05

.04

.05

.06

.07

.18

.08

.11

.10

.09

0.05

.50

1.38

1959.

Formula Best Est Formula Best Est.

Jan.

0.15

0.04

Feb.

.09

Mareh

.05

Apri1

.05

May

$.3 ?$

June

.07

July

.13

Aug .

.40

Špept.

.08

oct.

.10

Nov.

.02

Dec.

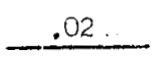

TOTAE

$$
1.53
$$

. 21

0.09

.15

.24

.09

$\begin{array}{rr}\text { Pre-1954 } & 0.45 \\ 1954 & 1.95 \\ 1955 & .24 \\ 1956 & .83 \\ 1957 & 1.38 \\ 1958 & 1.53\end{array}$

Total 
1254 Formula Best Est.

Jan.

0.0

Feb.

March

April

May

June

Juty

Aug .

Sept.

Oct.

Nov:

Dec.

TOTAL.

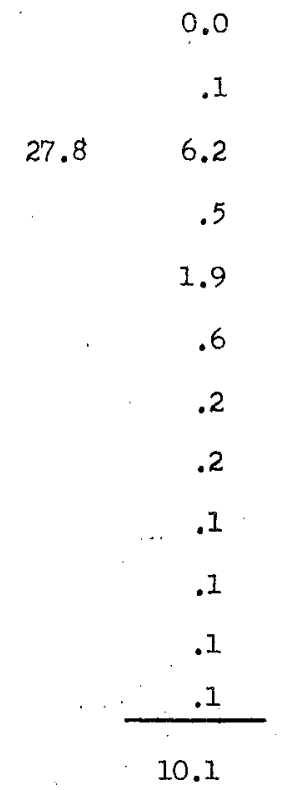

1955 Formula Best Est. $0.0 *$

.1 $.0 *$ $.0 *$ $.0 *$ $.0 *$ $.0 *$

$.0 *$

$.0 *$

$.0 *$

i

.0

0.2
1956 Eormula Best Est.

$0.1 *$

.0

$.1 *$

$.0 *$

.1

.4

.3

.1

.3

.1

.0

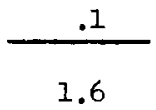

1952

Formule Best Est.

0.1

.1

.1

.1

.1

.3

.1

.2

.2

.2

.1

.9

2.5

1958

Jan.

Feb.

March

April

May

June

July

Aug.

Sept.

Oct.

Nov.

Dec.

TOTAL
0.3

.1

.1

.1

.8

.2

.3

1.0

.2

.3

.1

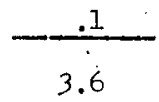

a Best Est.

$$
0.1
$$

.1

.4

.2

.3

.4

.2

\begin{tabular}{rr} 
Pre-1954 & 1.4 \\
1954 & 10.1 \\
1955 & 0.2 \\
1956 & 1.6 \\
1957 & 2.5 \\
1958 & 3.6 \\
1959 & \\
\hline
\end{tabular}

Totel 


\section{ESTIMATE OF Sr 90 DEPOSITION FROM GUMMED FILM}

STATION

908

LOCATION WAKE ISIAAND

2954

Eormula Best Est.

$$
0.04
$$

Feb.

Merch

Apr11

May

June

July

Aug.

Sept.

Oct.

Nov.

Dec.

TOTAL
.05

.09

.08

.12

.08

.13

.07

.28

.15

.13

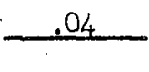

1.26
1955

Formula Best Est.

$0.05 *$

.11

$.21 *$

$.15 *$

$.09 *$

$.04 *$

$.04 *$

$.08 *$

$.07 *$

$.11 *$

.11

.32

1.38
1956.

Eormula Best Est.

$0.30 *$

.50

$.22 *$

$.14 *$

1.03

.52

4.40

2.11

.24

.22

.29

.16

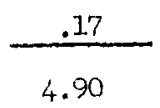

2.93
0.22

.28

2952

Formula Best Est.

.21

.22

.22

.18

.39

.20

.23

.27

.24

.27

958

1259

Formula Best Este Formula Best Est.

Jan.

0.21

0.55

Feb.

.19

.57

March

.39

ApriI

May

.58

.29

June

1.08

July

2.75

1.91

Aug .

.63

Sept.

.35

oct.

.22

Nov.

.17

Dec.

TOTAI.

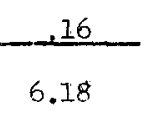

.54

1.01

.81

.34

.44

\begin{tabular}{rr} 
Pre-1954 & 1.03 \\
1954 & 1.26 \\
1955 & 1.38 \\
1956 & 4.90 \\
1957 & 2.93 \\
1958 & 6.18 \\
1959 & \\
\hline
\end{tabular}

Total 


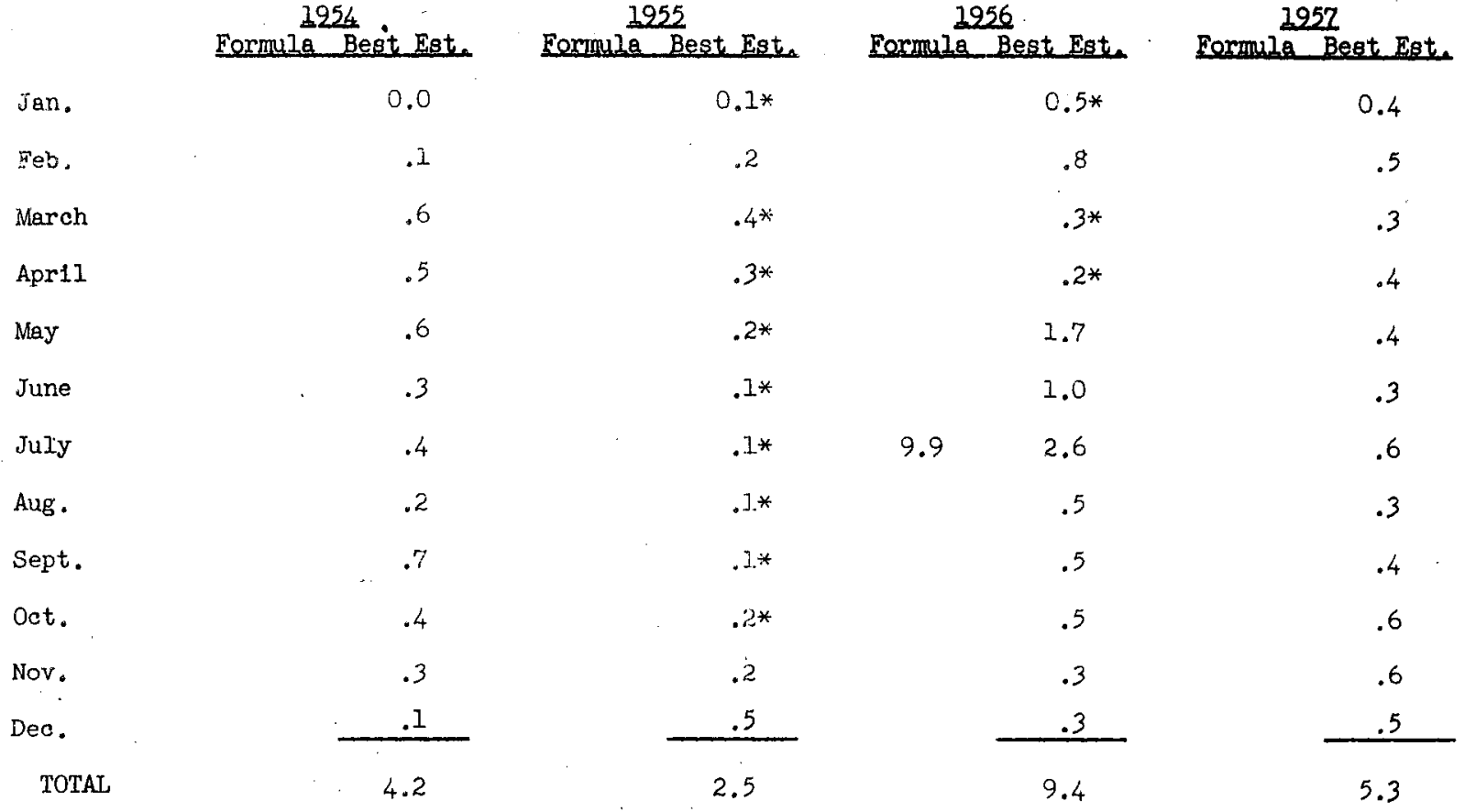

Formula Best Est. Formula Best Est.

$\begin{array}{lr}\text { Jan. } & 0.4 \\ \text { Feb. } & .3 \\ \text { March } & .8 \\ \text { Apr11 } & 1.0 \\ \text { May } & .6 \\ \text { June } & 2.1 \\ \text { July } & 4.9 \\ \text { Aug. } & 1.7 \\ \text { Sept. } & .8 \\ \text { Oct. } & .7 \\ \text { Nov. } & .5 \\ \text { Dec. } & -.4 \\ \text { TOTAL } & 14.2\end{array}$

\section{2}

1.1

1.0

1.8

1.4

.6

.7

\begin{tabular}{rr} 
Pre-1954 & 4.0 \\
1954 & 4.2 \\
1955 & 2.5 \\
1956 & 9.4 \\
1957 & 5.3 \\
1958 & 14.2 \\
1959 & \\
\hline
\end{tabular}


ESTIMATE OT Sr 90 DEPOSITION FROM GUMMED FILM

STATION \# .911

LOCATION TRUK, CAROLINE ISLANDS

$\frac{2254}{B e}$

Formula Best Estin

\begin{tabular}{lr} 
Jan. & 0.04 \\
Feb. & .02 \\
March & 1.39 \\
April & .55 \\
May & 1.52 \\
June & .30 \\
July & .13 \\
Aug. & .10 \\
Sept. & .04 \\
Oct. & .02 \\
Nov. & .10 \\
Deo. & .06 \\
\hline Total & 4.27
\end{tabular}

1955 Formule Best Est.

$0.09 *$

.07

$.05 *$

$.10 *$

$.05 *$

$.07 *$

$.02 *$

$.05 *$

$.04 *$

$.05 *$

.09

.20

.88 $\frac{1256}{\text { Rormula Best. Est. }}$

$0.42 *$

.13

$.27 *$

$.23 *$

$1.48 \quad 1.05$

$4.10 \quad 2.22$

4.07

1.74

.30

.27

.05

.11

.13

6.81
1957

Formula Best Est.

0.14

.21

.08

.09

.12

.13

.38

.21

.16

.10

.09

24

1.95

Formula Best Este Formula Best Est.

$\begin{array}{lrc}\text { Jen. } & & 0.10 \\ \text { Feb. } & : 04 \\ \text { March } & .07 \\ \text { Apr11 } & .22 \\ \text { May } & 4.89 & 3.40 \\ \text { June } & 10.20 & 4.67 \\ \text { July } & 3.80 & 3.73 \\ \text { Aug. } & & .55 \\ \text { Sept. } & & .30 \\ \text { Oct. } & & .18 \\ \text { Nov. } & & .09 \\ \text { Dec. } & & -20 \\ \text { TOTAL } & & 13.49\end{array}$

0.39

.97

.62

.58

.33

$\therefore .19$

.19

$$
\begin{array}{rr}
\text { Pre-1954 } & 1.51 \\
1954 & 4.27 \\
1955 & .88 \\
1956 & 6.81 \\
1957 & 1.96 \\
1958 & -3.49 \\
1959 & \\
\hline
\end{array}
$$

Total 


\section{ESTIMATE OF INFINITY $y$ DOSE FHOM GUMMED FILM}

1954. Eormula Best Est.

Jan.

Feb.

March

Apr11

May

June

July

Aug.

Sept.

oct.

Nov.

Dec.

TOTAL
0.0

.0

10.9

3.5

43.1

9.5

1.1

.4

.1

.1

.2

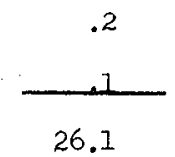

Formula Best Est.

$0.2 *$

.1

$.1 *$

$.2 *$

$.1 *$

$.1 *$

$.0 *$

.J.*

...*

.1*

.1

.3

1.5
1956

Formula Best Est.

$0.7 *$

.2

$.4 *$

$.3 *$

$2.9 \quad 1.7$

8.2

5.2

14.0

5.2

.7

.4

.1

.2

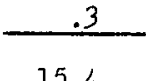

1957

Eormule Best Est.

0.2

.3

.1

.2

.2

.2

.6

.3

.3

.2

.2

.5

3.3
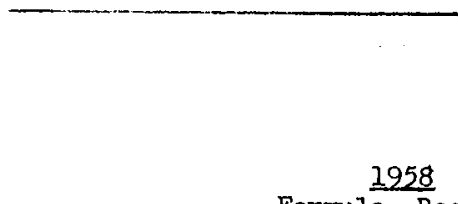

Formula Best Est. Formula Best Est.

1952

Jan.

0.2

0.8

Feb.

.1

1.9

March

.1

1.2

April

.4

May

9.6

6.6

June

19.3

9.1

July

10.2

8.2

Aug.

1.4

Sept.

.7

oct.

.5

Nov.

- . 3

Dec.

TOTAL

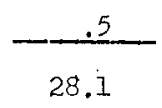

1.0

.6

.3

.3

\begin{tabular}{rr} 
Pre-1954 & 5.6 \\
1954 & 26.1 \\
1955 & 1.5 \\
1956 & 15.4 \\
1957 & 3.3 \\
1958 & 28.1 \\
1959 & \\
\hline
\end{tabular}

Total 


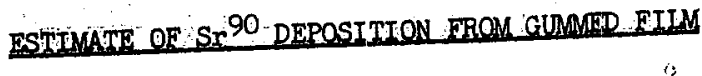

STATION :

LOCATION PONAPE, MARSHALL ISLANDS

\section{$1955 \quad 1956$}

Cormila Best Bat. Formula Best Esti Formia Beat Este Formula Best Est

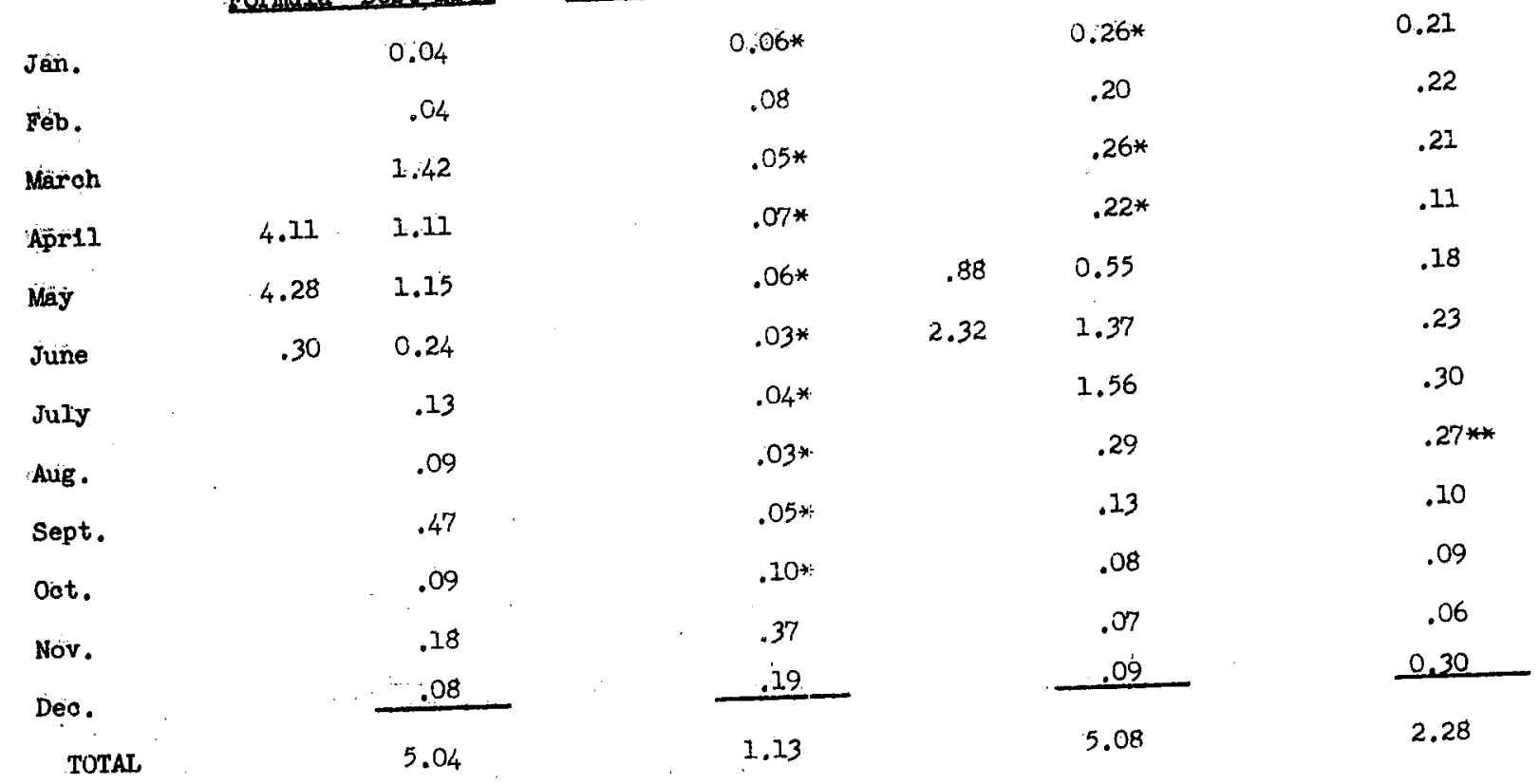

1958.1959

Formila Best Eat. Formila Best Este.

$\begin{array}{lrr}\text { Feb. } & .14 \\ \text { March } & .18 \\ \text { April } & & .45 \\ \text { May } & 11.77 & 4.96 \\ \text { June } & 6.31 & 3.84 \\ \text { JuIy } & & 2.00 \\ \text { Aug. } & .42 \\ \text { Sêpt. } & & .93 \\ \text { Oct. } & & 31 \\ \text { Nov. } & & 19 \\ \text { Dec. } & & 13.13 \\ \text { ToTAL } & & \end{array}$

$0: 64$

1.30

.85

1. 17

.54

.17

.28

.24

.17

11

\begin{tabular}{rr} 
Pre-1954 & 4.11 \\
1954 & 5.04 \\
1955 & 1.13 \\
1956 & 5.08 \\
1957 & 2.28 \\
1958 & 13.60 \\
1959 & \\
\hline
\end{tabular}

Total

.09 
1254 Formula Best Este.

Jan.

Feb.

March

Apr11

May

June

July.

Aug.

Sept.

Oct.

Nov.

Dec.

TOTAL

$$
0.0
$$

.0

9.6

28.3

29.3

1.2 .

0.9

.4

.2

1.2

.2

.4

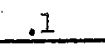

26.2

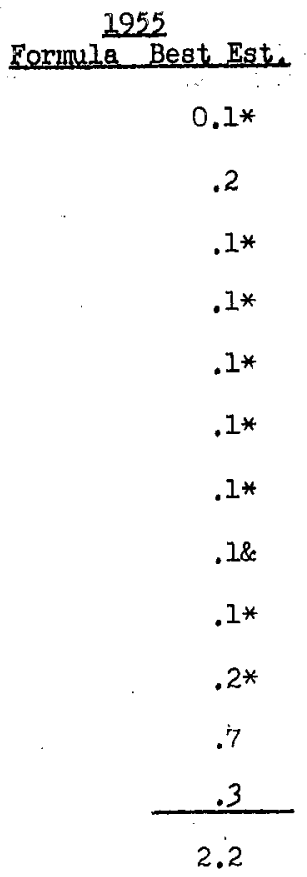

Formula Best Est.

$0.4^{*}$

.3

$.4^{*}$

.3*

$1.9 \quad 1.1$

6.5

2.5

3.8

.6

.3

.2

.I

.2

10.2
1957

Formula Best Est.

0.4

.4

.3

.2

.3

.4

.5

$.4 * *$

.2

.2

.1

0.5

3.9

1958

Formula Best Este Formula Best Est.

Jan.

Feb.

March

April

May

June

July

Aug.

Sept.

Oct.

Nov.

Dec.

TOTAL
0.1

.2

.4

.8

23.29 .8

$13.5 \quad 7.6$

5.5

1.1

2.1

.8

.3

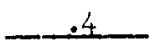

29.3
1.4

2.6

1.6

2.1

.9

.3

.5

.4

.3

.2

\begin{tabular}{rr} 
Pre-1954 & 16.5 \\
1954 & 26.2 \\
1955 & 2.2 \\
1956 & 10.2 \\
1957 & 3.9 \\
1958 & 29.3 \\
1959 & \\
\hline
\end{tabular}

Total 
STATHON 913

LOCATTON KOROR; PATAU ISLANDS

1954.

256

1957

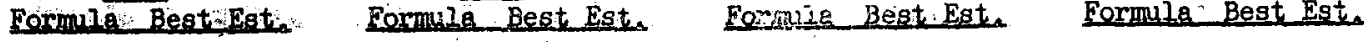

Tis:

0.04

$.04 *$

$0.13 *$

0.08

Seb:

.06

.04

.07

.11

Maroh

.92

$.05 *$

Apr11:

.95

$.03 *$

$.15 *$

.04

May

.93

$.05 *$

June

.17

Jury:

.08

Aug :

.10

Sept.

.09

Oat.

.07

Nov.

.05

Déo.

.02

$.03 *$

$.01 *$

$.12 *$

.10

TOTAL

3.48

$.02 *$

$.05 *$

.19

.13

.41

.08

1.20

.22

.16

.15

$.0 ?$

.16

$.05 *$

.28

.14

.13

.13

.25

.05

.21

.19

.69

2.92

1.44

1958

Formula Best Egt.

1959

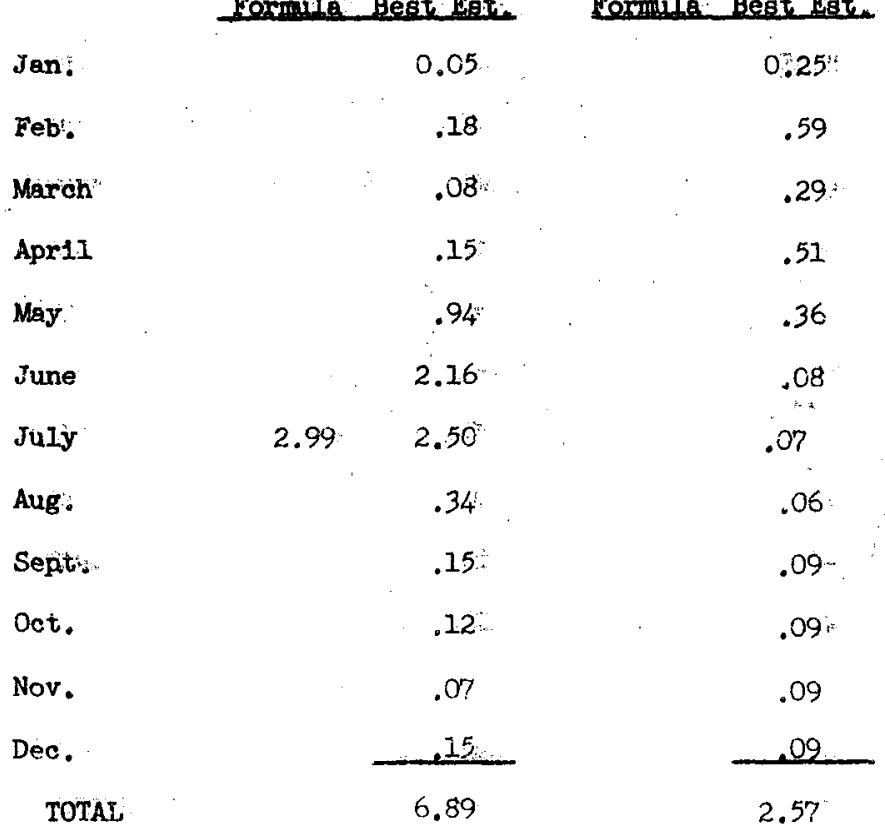




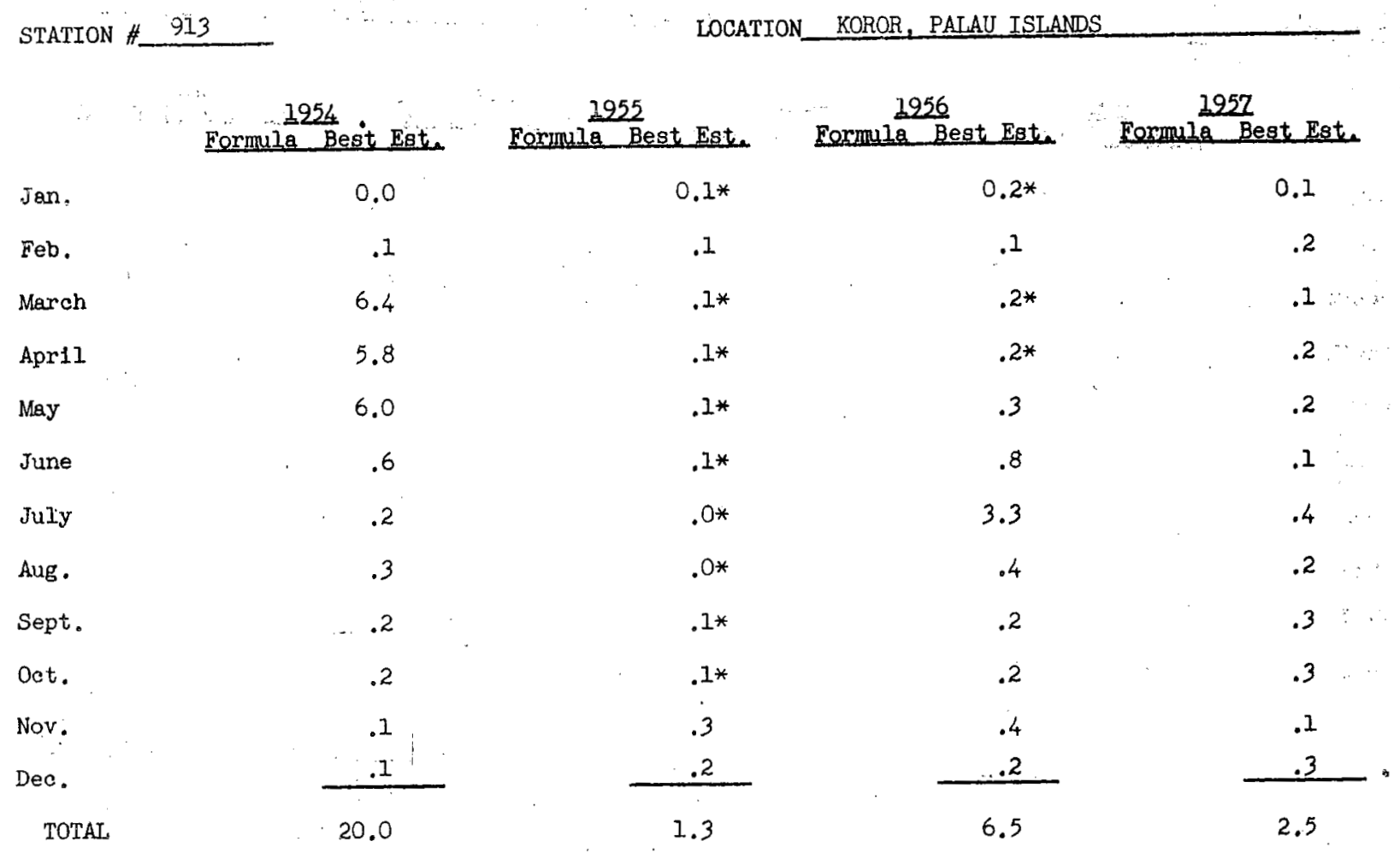

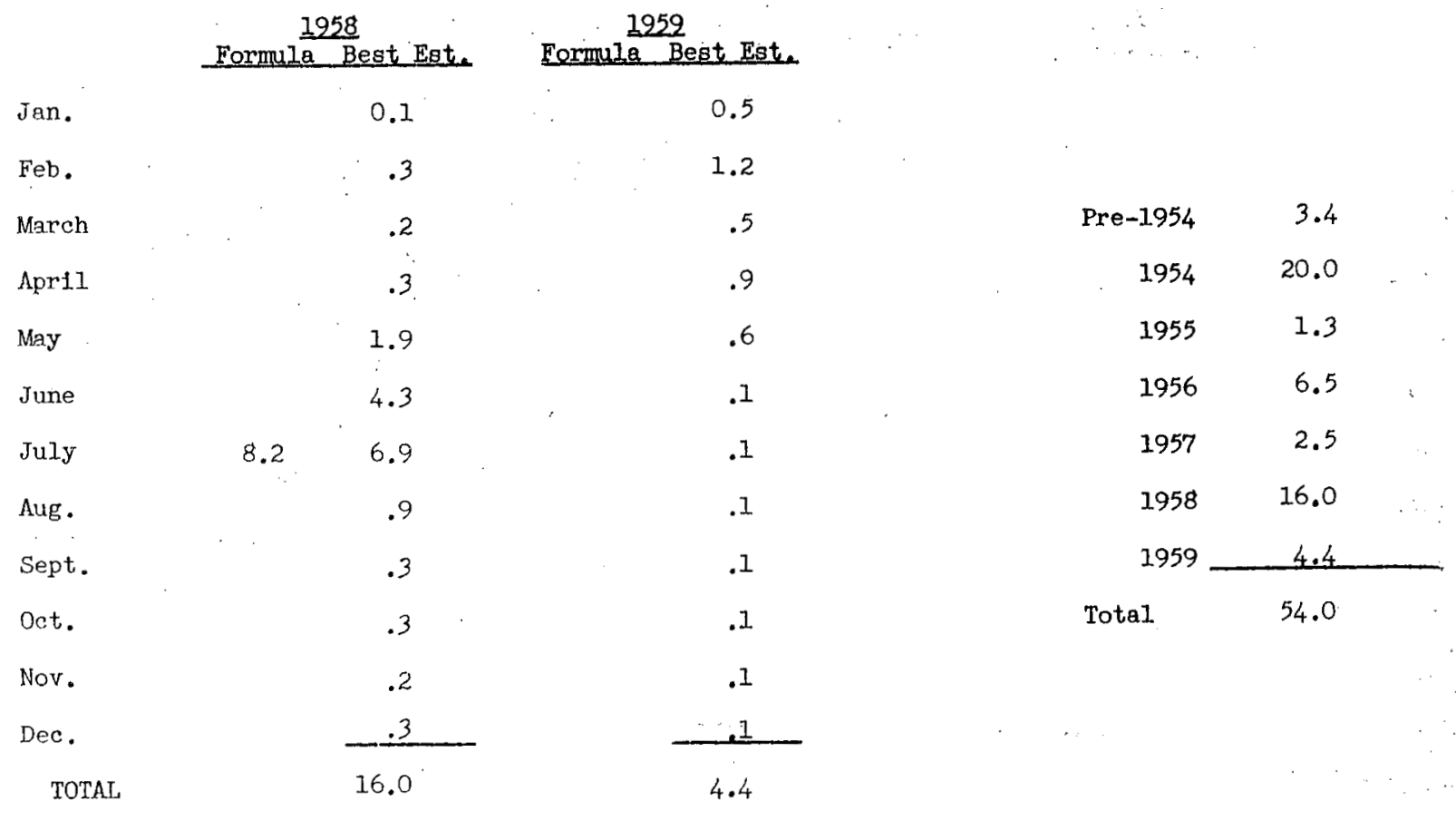


STATION \#-912

LOCATION_ YAP, PAIAU ISIANDS

$\therefore-1954 \quad 0 \quad 1955$

Eormula Best Este.

Jan.

Feb.

March

April

May

June

July

Aug .

Sept.

oot.

Nov.

Deo.

TOTAL
0.01

.04

1.27

4.04

1.14

.49

.10

.10

.06

.07

.15

.08

.05

3.56
1.256.

Eormula Best Est.

$$
0.32 *
$$

Formula Best Est.

$$
0.05 *
$$

$.04 *$

$.10 *$

$.05 *$

$.03 *$

$.05 *$

$.03 *$

$.06 *$

$.03 *$

$.08 *$

.11

.10

0.72

\begin{tabular}{l}
.05 \\
$.17 *$ \\
$.10 *$ \\
.62 \\
.45 \\
.91 \\
.08 \\
.09 \\
.02 \\
.15 \\
.05 \\
\hline 3.01
\end{tabular}

0.06

.09

.09

.11

.16

.12

.26

.17

.19

.08

.09

.73

2.20

1958

1259.

Formula Best Est. Formula Best Est.

Jan.

0.11

0.40

Feb.

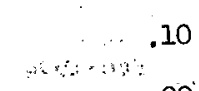

March

.09

April

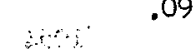

May

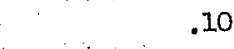

June.

$3.03 \quad 1.82$

1,08

.42

.48

July

$5.25 \quad 3.30$

.44

.09

.08

Aug .

$3.26 \quad 1.45$

Sept. ... $\quad .67$

.12

.11

oct.

.11

Nov.

.06

Dec.

TOTAL

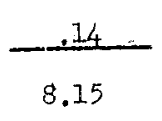

.14

\begin{tabular}{rl} 
Pre-1954 & 2.30 \\
1954 & 3.56 \\
1955 & 0.72 \\
1956 & 3.01 \\
1957 & 2.20 \\
1958 & 8.15 \\
1959 & 3.76 \\
\hline Total & 23.7
\end{tabular}

.19

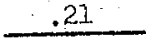

3.76 


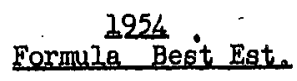

Jan.

Feb.

March

Apr11

May

June

July

Aug.

Sept.

Dot.

Nov:

Dec.

TOTAL

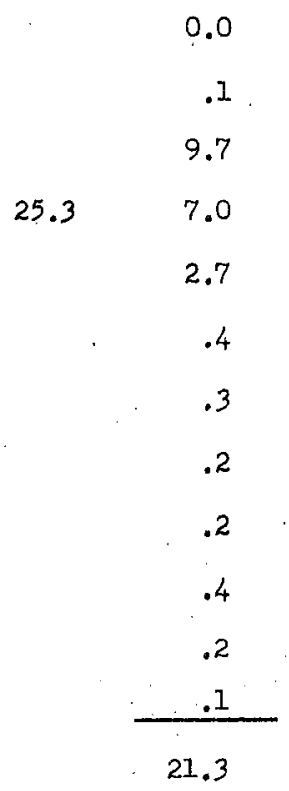

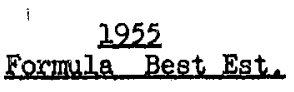

$0.1 *$

$.1 *$

$.2 *$

$.1 *$

.2*

$.1 *$

$.1 *$

. 1*

.1*

.1*

.2

.2

1.5
2956

Eormula Best Eat. Eormula Best Est.

$0.5 *$

0.1

.1

$.3 *$

.2

.1

$.2 * .2$

1.0

.3

.9

2.3

.2

.2

.1

.3

.1

6.2
.2

.4

.3

.4

.2

.2

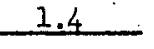

4.0

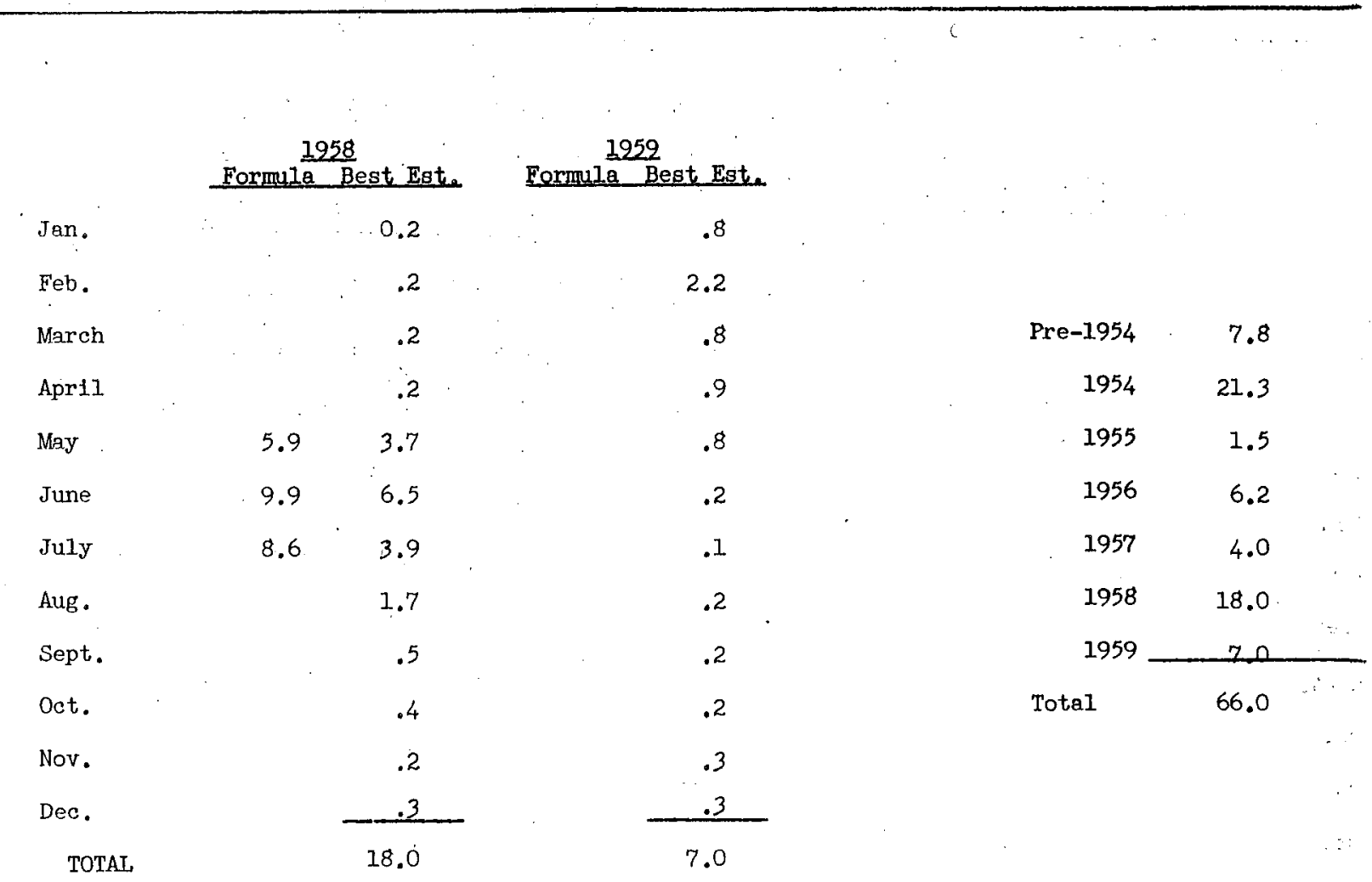


STATION \# 914

1954

Eormula Best Est.

Jan.

Feb.

Maroh

April

May

June

JuIy

Aug.

Sept.

Dot.

Nov.

Dec.

TOTAL

$$
0.04
$$

.04

.04

.85

.15

.08

.09

.06

.04

.21

.06

.05

1.71
1955 Formula Best Este.

$0.09 *$

.15

$.12 *$

$.06 *$

$.09 *$

$.06 *$

$.04 *$

$.06 *$

$.05 *$

.10*

0.13

.20

1.15

LOOATION: LIHUE, HAWAII

1952

Cormula Best Esti.

Formula Best Est.

0.39*

0.62 .

.32

.65

.36*

.26

$.10 *$

.36

.44

0.33

.40

.24

1.00

.40

.15

.43

.60

.28

.25

.17

.18

.17

.46

.49

4.65

4.40

2958

Formute Best Esten Eormula Best Est.

Jan.

0.27

1.92

Feb.

.24

1.91

March

1.11

1.63

Apr11

.67

1.78

May

.50

1.20

.40

June

.68

July

.64

.29

Aug.

.32

.48

Sept.

.22

.28

oct.

.21

.18

Nor.

.19

.09

Dec.

$\frac{.80}{5.85}$

TOTAL

5.85 
1254

Formula Best Est.

Jan.

Feb.

March

Apri1

May

June

July

Aug.

Sept.

Oct.

Nov.

Dec.

TOTAL
0.0

.1

.3

5.1

.8

.3

.3

.2

.1

.5

.1

.1 .

7.9
1955 Formula Best Est.

$0.2 *$

.3

$.2 *$

$.1 *$

$.2 *$

$.1 *$

$.1 *$

$.1 *$

$.1 *$

$.2 *$

.2

.3

2.1
1956

Formula Best Est.

$0.6 *$

.5

$.6 *$

$.1 *$

.7

.8

2.1

.3

1.3

.5

.3

.9

8.7
1957

Formula Best Est.

1.1

1.1

.4

.6

.5

.4

.6

.7

.5

.4

.4

$.9=$

7.6
1958

Formula Best Est.

0.5

Jan.

Feb.

March

April

May

June

July

Aug.

Sept.

Oet.

Nov.

Dec.

TOTAI,
.7

.5

$-\frac{1.9}{12.5}$
1959

Formula Best Est.

4.1

3.9

3.1

3.2

2.1

.7

.5

.8

.4

.3

Pre-1954 2.2

$1954 \quad 7.9$

$1955 \quad 2.1$

$1956 \quad 8.7$

$1957 \quad 7.6$

$1958 \quad 12.5$

1959

Total
.1 


\section{ESTIMATE OF Sr 90 DEPOSITION FROM GUMMED. FILM}

STATION \#.916.

WOCATION HILO, HAWATI

Formu21 Best Est.

0.04

Jan.

Feb.

March

Apri1

May

June

JuIy

Aug.

Sept.

oct.

Nov.

Deo.

TOTAL
.03

.12

.86

$1.48 \quad 0.73$

.13

.10

.06

.06

.11

.05

.05

2.34
2955 Formule Best-Est:

$0.17 *$

.55

$.28 *$

$.21 *$

$.31 *$

$.22 *$

$.20 *$

$.12 *$

$.11 *$

$.16 *$

.21

.19

2.73
1256: Formule Best Est. Eormula Best Est.

$$
0.47 *
$$

0.65

1,00

.71

$.84 *$

$.30 *$

1.22

1.04

1.65

.40

.61

.36

.26

.32

8.47
.76

.70

.71

.49

.83

.58

.43

.30

.25

.57

6.98

1958

Formula Best Est. Formila Best Est.

\begin{tabular}{lr} 
Jan. & 0.26 \\
Feb. & .33 \\
March & 1.47 \\
April & 1.95 \\
May & 1.33 \\
June & .75 \\
July & .63 \\
Aug. & .55 \\
Sëpt: & .34 \\
Oct. & .44 \\
Nov. & .51 \\
Dec. & -.73 \\
\hline TOTAL & 9.20
\end{tabular}

1.247

4.57

2.84

3.58

3.07

.94

.63

\begin{tabular}{rr} 
Pre-1954 & 1.02 \\
1954 & 2.34 \\
1955 & 2.73 \\
1956 & 8.47 \\
1957 & 6.98 \\
1958 & 9.20 \\
1959 & \\
\hline
\end{tabular}

Total 
STATION \# 916

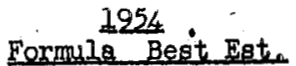

Jan.

Feb.

March

Apr11

May

June

JuIy

Aug .

Sept.

Oct.

Nov.

Dec.

TOTAL
0.0

.0

.7

5.0

9.4

4.1

.5

.3

.2

.2

.3

.1

.1

11.5
1955

Formula Best Est.

$0.4^{*}$

1.1

$.5 *$

$.4 *$

$.6 *$

$.4 *$

$.3 *$

$.2 *$

$.2 *$

$.3 *$

.4

.3

5.1
1956 Eormula Best Est. $0.7 *$

1.6

$1.3 *$

$.4 *$

1.9

2.1

3.4

.9

1.3

.7

.5

.6

15.4
1957

Formula Best Est.

1.1

1.2

1.2

1.3

1.2

.9

1.4 .

.9

.7

.6

.5

100

12.0

1958

1952

Formula Best Este Formula Best Est.

\begin{tabular}{lc} 
Jan. & 0.4 \\
Feb. & .6 \\
March & 2.9 \\
April & 3.4 \\
May & 2.6 \\
June & 1.4 \\
July & 1.7 \\
Aug. & 1.4 \\
Sept. & .8 \\
Oct. & 1.5 \\
Nov. & 1.4 \\
Dec. & 1.7 \\
\hline ToTAL & 19.8
\end{tabular}

3.1

9.1

5.4

6.5

5.3

1.6

1.0

\begin{tabular}{rr} 
Pre-1954 & 3.8 \\
1954 & 11.5 \\
1955 & 5.1 \\
1956 & 15.4 \\
1957 & 12.0 \\
1958 & 19.8 \\
1959 & \\
\hline
\end{tabular}

Total 


\section{ESTIMATE OF SY 90 DEPOSITION EROM GUMADO EILN:}

STATION \# 915

IOCATION HONOLULIS, HAWAII

Eormula Best Est.

Jan.

Feb.

Maroh

April

May

June

July

Aug.

Sept.

oot.

Nov.

Dec.

TOTAL
0.04

.03

.15

2.55

0.68

.38

.11

.11

.04

.04

.09

.05

.01

1.73
Eormula Best Est.

$0.04 *$

.16

$.11 *$

$.11 *$

$.09 *$

$.10 *$

$.07 *$

$.07 *$

$.04 *$

$.10 *$

.06

.17

1.12
2956 Eormule Best Eat.

$0.21 *$

.40

$.25 *$

$.24 *$

.23

1.06

1.01

.08

.22

.15

.14

.11

4.10
1957

Formule Best Est.

0.34

.51

.21

.28

.15

.18

.47

.27

.26

.12

$.20 * *$

$2.1 * *$

3.40

Formula Best Este Formili: Best Este.

Jan.

0.27

0.96

Feb.

March

.16

.89

Apri1

.51

May

.58

June

.38

July

.53

Aug.

.22

Sept.

.12

oct.

.19

1.48

1.08

.86

1.96

.23

$\therefore .10$

Nov.

.13

Dec.

.45

TOTAL

4.43

Pre-1954 0.49

$1954 \quad 1.73$

$1955 \quad 1.12$

$1956 \quad 4.10$

$1957 \quad 3.40$

19584.43

1959

Total 
STATION \#-915

LOCATION HONOLULU; HAWAII

1256 Formula Best Est:

Jan.

Feb.

March

April

May

June

Juİ̀

Aug.

Sept.

oct.

Nov.

Deo.

TOTAL
0.0

.1

.8

2.1

.4

.3

.1

.1

.2

.1

.0

8.1
$15.2 \quad 3.9$
2955 Formule Best Est:

$0.1 *$

.3

$.2 *$

$.2 *$

$.2 *$

$.2 *$

$.1 *$

$.1 *$

$.1 *$

$.2 *$

.1

3.

2.1
1256 Eormula Best Est:

$0.3 *$

.6

$.4^{*}$

$.4^{*}$

.4

2.1

2.1

.2

.5

.3

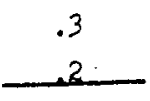

7.8

\section{2}

Formule' Best Est

0.6

.8

.3

.5

.3

.3

.8

.4

.4

.2

$.4 * *$

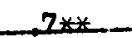

5.7

\begin{tabular}{|c|c|c|}
\hline & Formula $\frac{1958}{\text { Best Est. }}$ & Formula Best Est. \\
\hline Jan. & 0.4 & 2.0 \\
\hline Feb. & .3 & 3.0 \\
\hline March & 1.8 & 2.0 \\
\hline Apri1 & $: 9$ & 1.5 \\
\hline May & 1.1 & 3.4 \\
\hline June & .8 & .4 \\
\hline July & 2.4 & .2 \\
\hline Aug. & .6 & \\
\hline Sept. & .3 & \\
\hline oct. & .6 & \\
\hline Nov: & .4 & \\
\hline Dec. & $1.1 \ldots$ & $\ldots$ \\
\hline TOTA & 9. & \\
\hline
\end{tabular}

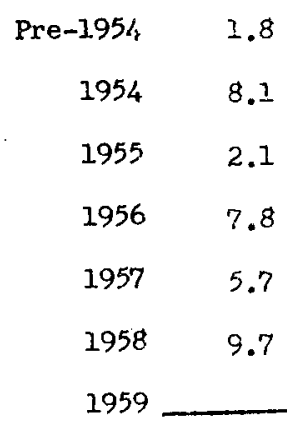


STATION \#

roaATIOL KWALAIEIN, MARSHALL ISIANDS

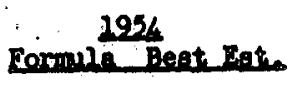

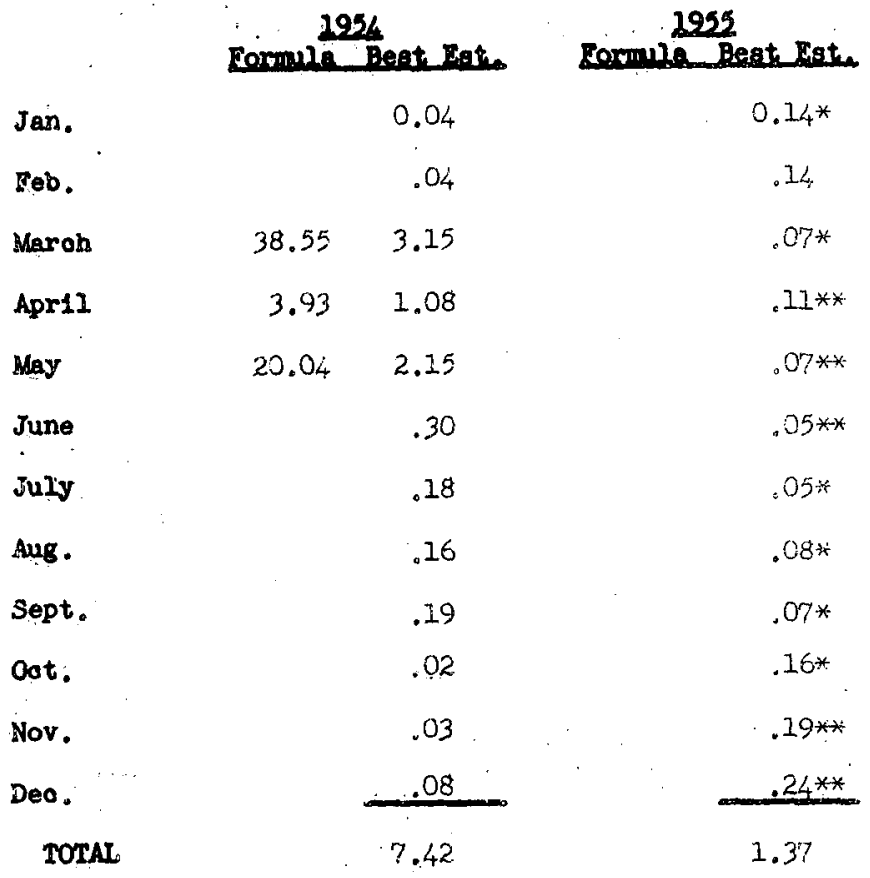

Formula Beativat.

$0.32 * *$

Cormin Begt Zet.

$\begin{array}{r}0.32 * * \\ .17 \\ .16 * \\ .43 * \\ 1.43 \quad 0.76 \\ 14.40 \quad 1.44 \\ 4.36 \\ .89 \\ .38 \\ .28 \\ .24 \\ .12 \\ \hline .55\end{array}$

0.16

.21

.10

.08

.07

.20

.24

.33

.20

.08

$.13 * *$

.16

1.96

2958

Formile Best Bat.

1952

Corrula Best zat.

0.08

$0.40^{\circ}$

Jan.

.26

Feb.

.10

March

.12

April

May.

12.13

5.91

1.31

June

1.64

July

.35

Aug .

.12

Oot.

.14

Nov.

Deo.

Torat.
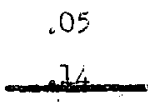

10.22

.52

.38

.70

.26

.13.

.16 .

.17 .

.05

.07

\begin{tabular}{rc} 
Pre-1954 & $\cdots$ \\
1954 & 7.42 \\
1955 & 1.37 \\
1956 & 9.55 \\
1957 & 1.96 \\
1958 & 10.22 \\
1959 & 3.09 \\
\hline
\end{tabular}

Total 
1954 Formula Besit Est.

Jan,

Feb.

March

Apri1

May

June

JuIy

Aug .

Sept.

oct.

Nov.

Dec.

TOTAL
0.0

.0

$314.3 \quad 25.0$

$26.3 \quad 6.5$

$133.2 \quad 13.2$

1.2

.6

.5

.5

.1

.2

.1

35.8
1955

Formula Best Est.

$0.3 *$

.3

. $1 *$

$.2 * *$

.1**

.1**

$.2 *$

. $1 *$

.1*

$.3 *$

$.4^{* * *}$

$.4 * *$

2.5
1956

Formula Best Est.

$0.5 * *$

.3

$.2 *$

$.6 *$

$10.6 \quad 2.0$

27.4

5.8

9.2

2.0

.9

.5

.5

.2

22.6
Formula Best Est.

$\therefore \quad 0.3$

1957

.3

.2

.1

.1

.4

.4

.5

.3

.2

$.3 * *$

3

3.4

Formula Best Est.

1959

Formula Best Eat.

Jan.

0.1

0.9

Feb.

.5

March

Apr11

.2

May

.2

June

24.3

12.9

July

2.5

4.4

Aug.

.9

Sept.

.3

oct.

.4

1.0

Nov.

.1

Dec.

TOTAL

$-.3$

22.8

.7

1.3

.5

.2

.3

.3

.1

.1

.2

.2

4.9

\begin{tabular}{rr} 
Pre-1954 & - \\
1954 & 35.8 \\
1955 & 2.5 \\
1956 & 22.6 \\
1957 & 3.4 \\
1958 & 22.8 \\
1959 & 4.9 \\
\hline
\end{tabular}

Total 